\title{
Reaction Kinetics of Hydroxyl Radical with Polycyclic Aromatic Hydrocarbon Precursors
}

Juddha Thapa

Follow this and additional works at: https://researchrepository.wvu.edu/etd

\section{Recommended Citation}

Thapa, Juddha, "Reaction Kinetics of Hydroxyl Radical with Polycyclic Aromatic Hydrocarbon Precursors" (2016). Graduate Theses, Dissertations, and Problem Reports. 6789.

https://researchrepository.wvu.edu/etd/6789

This Dissertation is protected by copyright and/or related rights. It has been brought to you by the The Research Repository @ WVU with permission from the rights-holder(s). You are free to use this Dissertation in any way that is permitted by the copyright and related rights legislation that applies to your use. For other uses you must obtain permission from the rights-holder(s) directly, unless additional rights are indicated by a Creative Commons license in the record and/ or on the work itself. This Dissertation has been accepted for inclusion in WVU Graduate Theses, Dissertations, and Problem Reports collection by an authorized administrator of The Research Repository @ WVU.

For more information, please contact researchrepository@mail.wvu.edu. 


\title{
Reaction Kinetics of Hydroxyl Radical with Polycyclic Aromatic Hydrocarbon Precursors
}

\author{
by \\ JuddhaThapa \\ Dissertation Submitted \\ to the Eberly College of Arts and Sciences \\ at West Virginia University \\ in partial fulfillment of the requirements for the degree of \\ Doctor of Philosophy in Physical Chemistry
}

\begin{abstract}
Approved by
Fabien Goulay, Ph.D., Committee Chairperson

Kenneth Showalter, Ph.D.

Michelle Richards-Babb, Ph.D.

Kung Wang, Ph.D.

Brian Anderson, Ph.D.

C. Eugene Bennett Department of Chemistry

Morgantown, West Virginia

2016

Keywords: Gas Phase Reactions, Free Radicals, Hydrocarbons, Rate Constant, Pulsed Laser

Photolysis, Laser Induced Fluorescence, Temperature and Pressure Range, Addition Intermediates
\end{abstract}

Copyright 2016 Juddha Thapa 


\section{Abstract \\ Reaction Kinetics of Hydroxyl Radical with Polycyclic Aromatic Hydrocarbon Precursors \\ JuddhaThapa}

The incomplete combustion of fuels inside of an internal combustion engines generates unwanted byproducts such as soot. Because of health and environmental concerns, soot formation has been a very active area of research in combustion chemistry. However, the mechanism of formation of soot is still not well understood. It has been proposed that the soot formation is initiated by the reaction of small free radicals with abundant hydrocarbon fuel molecules producing aromatic ring structures at high temperatures. These aromatic ring structures further react to form polycyclic aromatic hydrocarbons (PAHs) that are stable at the high temperatures of combustion environments. These PAHs collide and stick with each other forming dimers, trimers, tetramers, etc. Eventually such stable PAHs-stabilomers condense and transform into solid particles (soot). To minimize pollutants and increase the efficiency of engines, it is very important to understand the chemistry of the elementary reactions at the molecular level. The reactions of hydroxyl free radicals with polycyclic aromatic hydrocarbon precursor molecules are studied experimentally in a quasi-static gas cell using laser pump-probe spectroscopy. Hydroxyl free radicals are generated by pulsed laser photolysis (PLP) using the third (355 nm) or fourth $(266 \mathrm{~nm})$ harmonic of Nd:YAG laser and their concentration is monitored as a function of laser delay-time using a frequency-doubled tunable dye laser perpendicular to the photolysis laser. The off-resonance fluorescence from the hydroxyl free radicals at $310 \mathrm{~nm}$ is collected by a photomultiplier tube (PMT) placing it orthogonal to the photolysis and probe laser beams.

The reactions of hydroxyl radicals $(\mathrm{OH})$ with phenylacetylene and fulvenallene have been investigated from $298 \mathrm{~K}$ to $450 \mathrm{~K}$. The concentrations of the hydrocarbon reactants are measured using FTIR spectroscopy and UV absorption. The room temperature reaction rate of the $\mathrm{OH}+$ phenylacetylene reaction is measured to be $8.75( \pm 0.73) \times 10^{-11} \mathrm{~cm}^{3} \mathrm{~s}^{-1}$. The reaction rate coefficient is pressure and temperature independent over the 1-7.5 Torr and 298-423 K pressure and temperature ranges. The rate coefficient is larger than that expected based solely on association with the aromatic ring, which suggests reaction with the triple bond. For the $\mathrm{OH}+$ fulvenallene reaction, the room temperature rate coefficient is found to be $8.8( \pm 1.7) \times 10^{-12} \mathrm{~cm}^{3} \mathrm{~s}^{-1}$ with the negative temperature dependence. The comparison of the experimental rate coefficients with the calculated abstraction rate coefficients suggests that over the experimental range, association of hydroxyl radical $(\mathrm{OH})$ to fulvenallene plays the significant role toward the formation of PAH precursors. 
"I dedicate this dissertation to my parents

Dhan Bahadur Thapa and Krishna Kumari Thapa, who sacrificed their life for

my education despite they themselves having no formal education." 


\section{Acknowledgments}

I would like to gently close my eyes for a moment and remember all the helping hands lent to me throughout my Ph.D. education.

Dr. Fabien Goulay, you are the great adviser who always motivated and mentored me in all the circumstances. I am truly grateful to you for allowing and trusting me to use all the new instruments in the new laboratory despite knowing I was a new researcher. Thank you very much for crafting a confident researcher in me through your adept advice. Your comments and suggestions in all the steps were always precious to proceed in scientific research. Thank you both for pointing my limitations and suggesting the ways forward. I am also grateful to all my laboratory mates in Goulay laboratory for their help and friendship.

I would like to express my appreciation to Sherman Adams for making wonderful glass apparatus for the experiments; Allen Burn for making all the metal and teflon equipment; and Philip Tucker for helping in all electrical and electronic equipment. Philip Tucker-your marks will always be deeply felt at Goulay Laboratory.

Thank you very much the friends in Dr. Wang laboratory for letting me use the oven or clean my dirty glassware.

I am also extremely grateful to my Ph.D. committee members, Dr. Kenneth Showalter, Dr. Michelle Richards-Babb, Dr. Kung Wang, and Dr. Brian Anderson for their time, advice, and encouragement.

I would also like to express my deepest gratitude to my parents for their unconditional support, sacrifice, and encouragement throughout my life. You always backed me up and never let me down. You have the invaluable role in making me the first generation college student. I 
always thank God for having parents like you who always put my education the first priority. I'm also thankful to my wife Nirmala Thapa for loving support. I experienced difficulty having to live away from the family, and I'm thankful that you always provided the emotional strength to pursue my education The birth of my son, Aarush, last year not only reminded me of my responsibilities and deeper joys of life but also provided the relaxing and rejuvenating moments in the evening when I returned home after a long research or teaching day.

Lastly, I am very much grateful to the student organization "Nepalese Students and Scholars Association, West Virginia University (NSSAWVU)" for consolidating all the Nepalese students at WVU and their families. All the social events and camping were great platform to be together and share common interests. Those events were really recreational and became the place to relieve stress. I was able to overcome frustrations resulted from the failure of some research steps many times and hectic schedule in the personal and college lives. This organization really made me feel at home away from home. 


\section{Table of Contents}

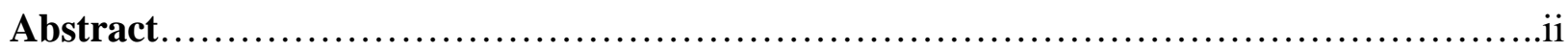

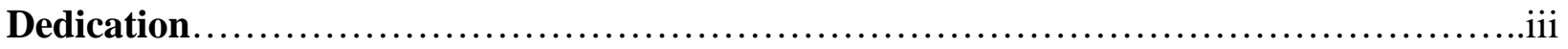

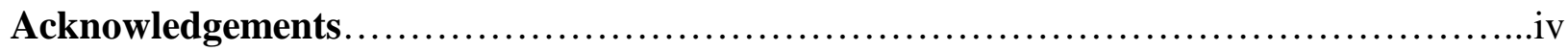

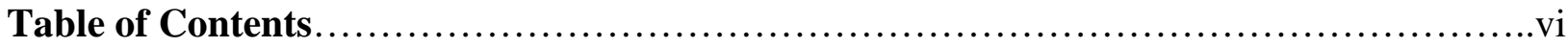

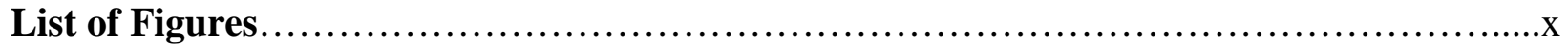

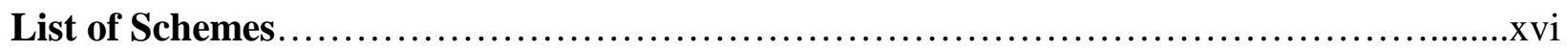

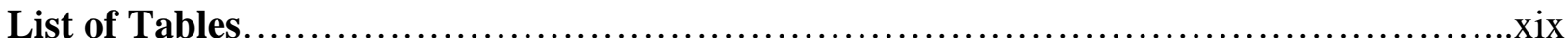

List of Symbols and Abbreviations........................................................

Chapter 1: Introduction ....................................................................

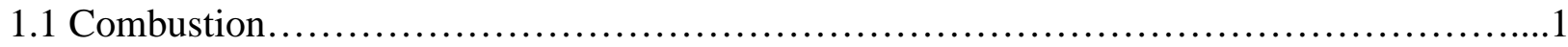

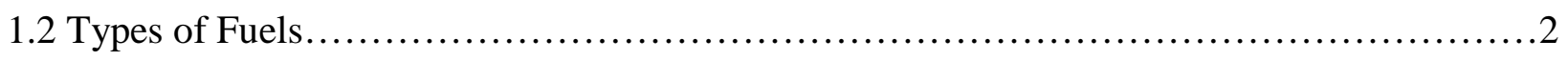

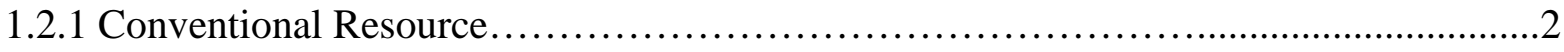

1.2.1.1 Petroleum.....................................................................

1.2.1.2 Natural Gas............................................................

1.2.2 Non-Conventional Source..........................................................

1.2.2.1 Biofuels and its Types..................................................

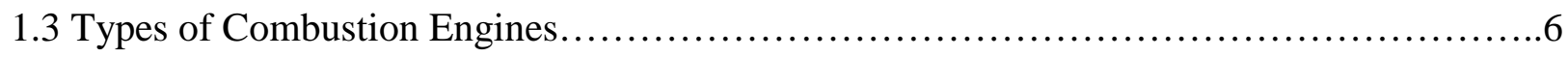

1.4 Combustion Byproducts...................................................................

1.5 Low Temperature Combustion (LTC): A Way to Improve Combustion Device..............12

1.6 Combustion Chemistry .................................................................

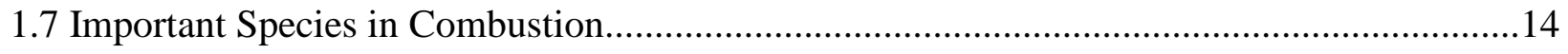

1.8 OH Radical Reaction Scheme...................................................... 15

1.8.1 The Temperature Dependence of the Different Reaction Channels.....................17

1.8.2 The Effect of Pressure on the Stabilization of the Adduct............................19 
1.9 First Ring Formation and Carbon Molecular Growth.................................20

1.10 Formation of Polycyclic Aromatic Hydrocarbons (PAHs) ............................22

1.10.1 The HACA Mechanism.................................................... 24

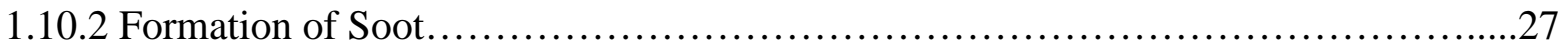

1.11 Resonance-Stabilized Radicals (RSRs): Alternative Pathways for PAH Formation........28

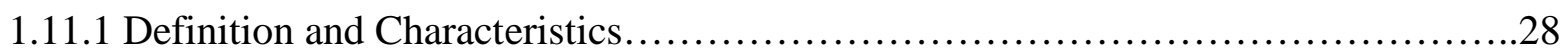

1.11.2 Current Knowledge on Resonance-Stabilized Radical Reactivity...................30

1.11.3 Previous Experiments and Computational Studies on Resonance-Stabilized

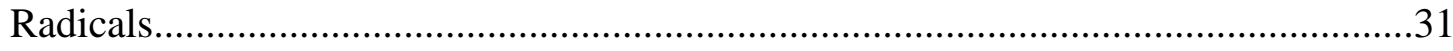

1.11.4 What Else do We Need to Know.............................................

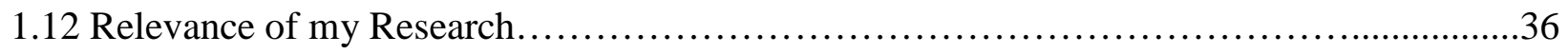

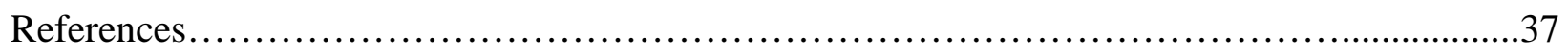

Chapter 2: Experimental Methods................................................42

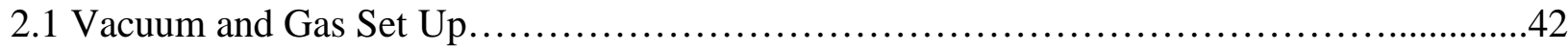

2.1.1 The Reaction Chamber...................................................42

2.1.2 Vacuum Pump and Gas Injection.............................................

2.1.2.1 Vacuum Pump.........................................................44

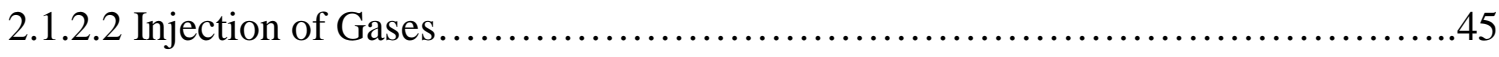

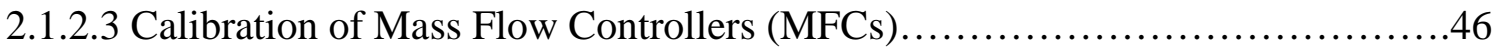

2.1.2.4 Determination of Buffer Gas Total Flow Rate and Average Linear Velocity.....47

2.1.2.5 High Temperature Set Up..........................................49

2.2 The Pulsed Laser Photolysis-Laser Induced Fluorescence (PLP-LIF) Technique...........49

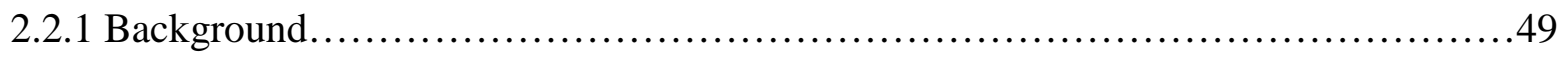

2.2.2 Pulsed Photolysis........................................................ 51

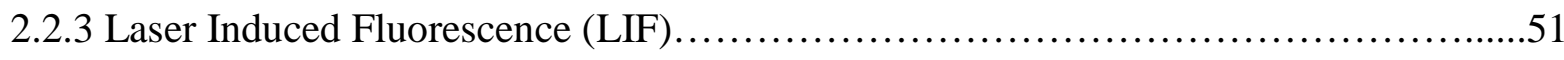




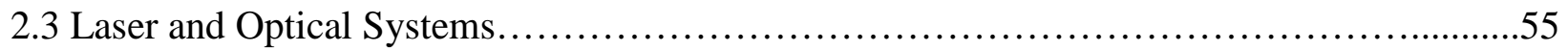

2.3.1 Nd:YAG and Harmonic Generation............................................58

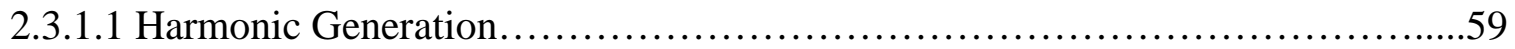

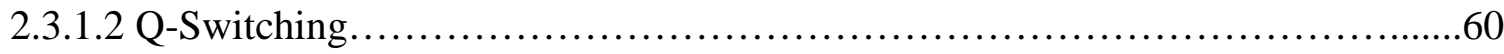

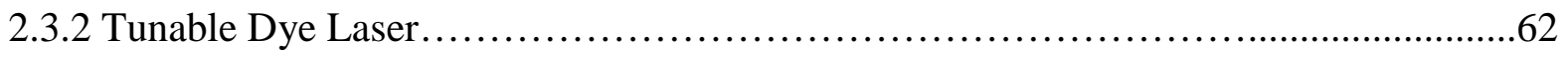

2.3.3 Photomultiplier Tube (PMT) ......................................................65

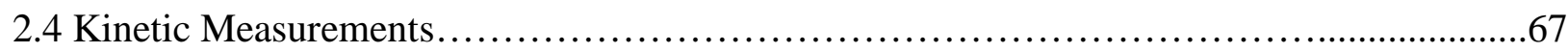

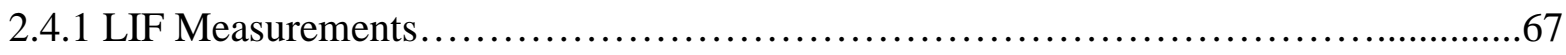

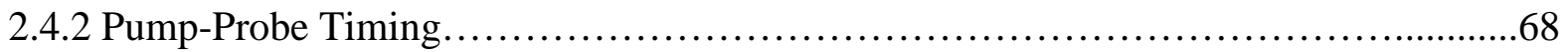

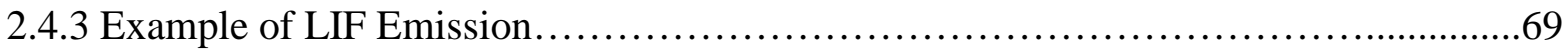

2.4.4 Pseudo First Order Approximation.......................................................

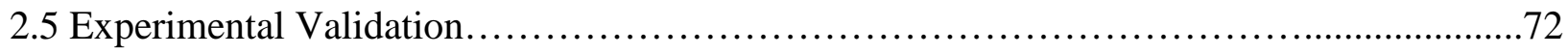

2.5.1 OH + Propene, Butene, Acetaldehyde, and Benzene..................................73

2.5.2 Computational Method.......................................................... 74

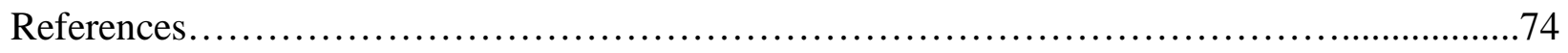

Chapter 3: Reaction of the OH Radical with Phenylacetylene................................77

3.1 OH Radical Reaction with Aromatic Molecules..............................................77

3.2 Phenylacetylene in Combustion...................................................................

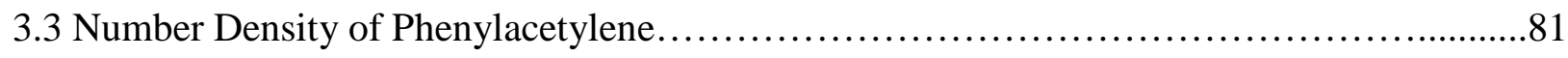

3.4 Determination of the Phenylacetylene Absorption Cross-Section at $266 \mathrm{~nm} . . . \ldots \ldots \ldots \ldots \ldots . . . . . . . . .83$

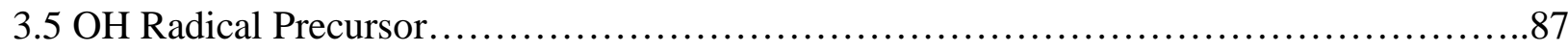

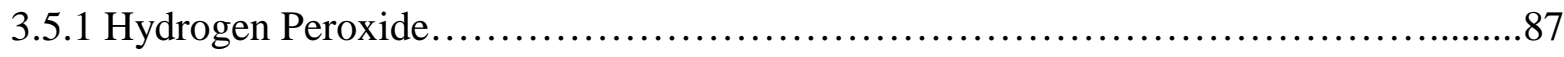

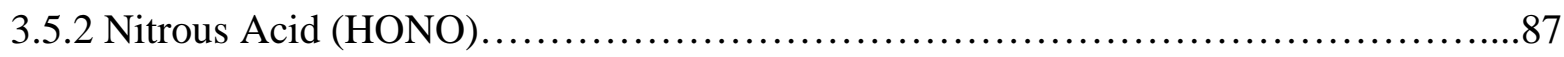

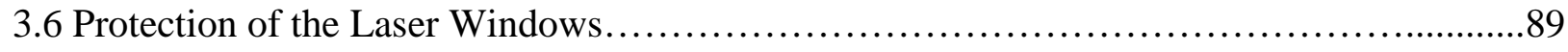

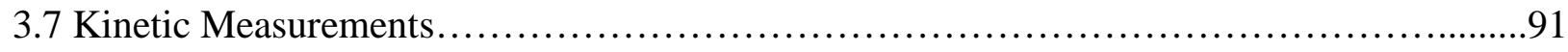


3.7.1 Pseudo-First Order Kinetics..........................................................

3.7.2 Laser Power Dependence of Phenylacetylene...........................................93

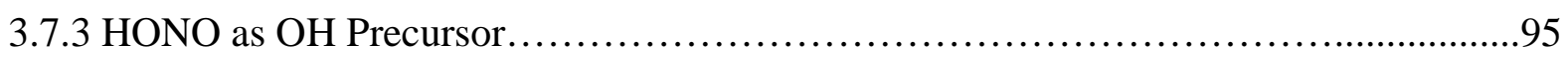

3.8 Pressure and Temperature Dependence of Phenylacetylene + OH Kinetics.........................96

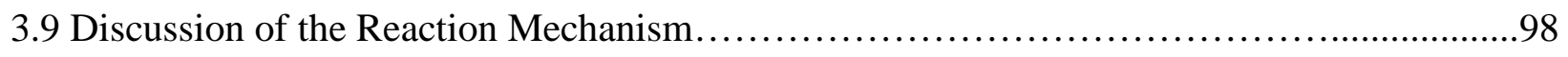

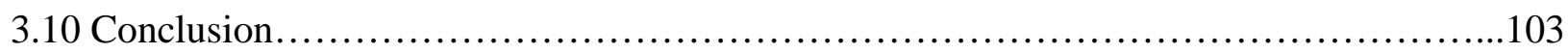

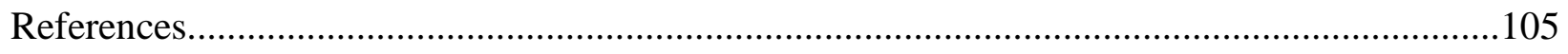

Chapter 4: Reaction of the $\mathrm{OH}$ Radical with Fulvenallene................................107

4.1 Fulvenallene in Combustion Environments..........................................107

4.2 Fulvenallene Synthesis and Characterization................................................108

4.2.1 Properties of Fulvenallene and its Precursors.........................................108

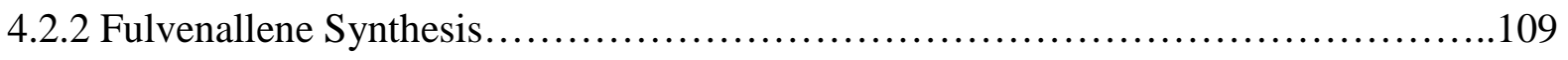

4.2.3 Liquid Phase NMR Spectroscopy.................................................112

4.2.4 Gas Phase FTIR Spectroscopy.................................................114

4.2.5 Fulvenallene Purity ........................................................... 118

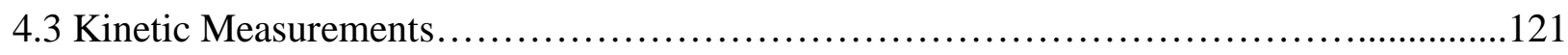

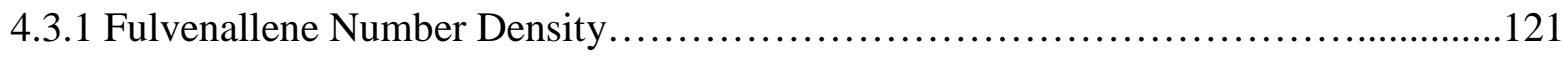

4.3.2 Pseudo-First Order Kinetics......................................................122

4.3.3 Temperature Dependence of the Rate Constant..........................................124

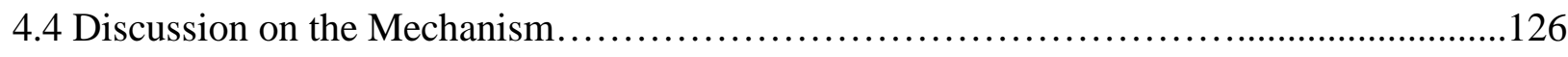

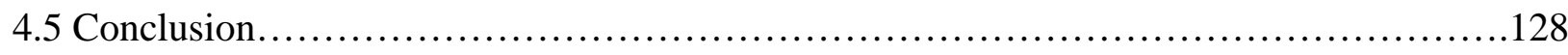

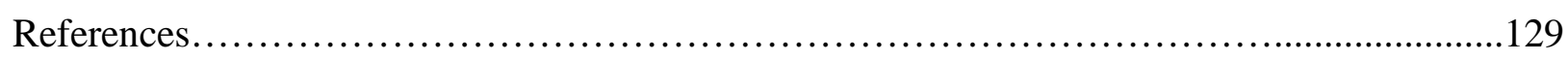

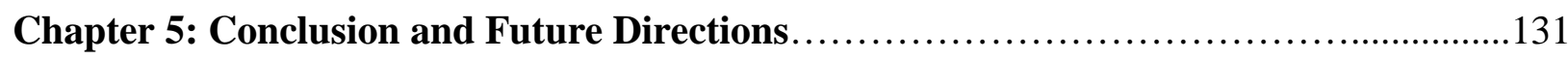

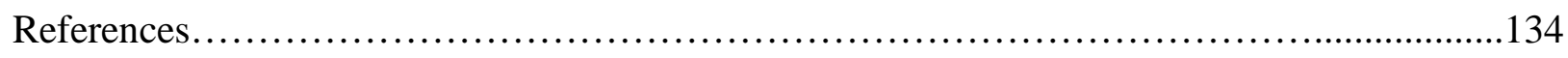

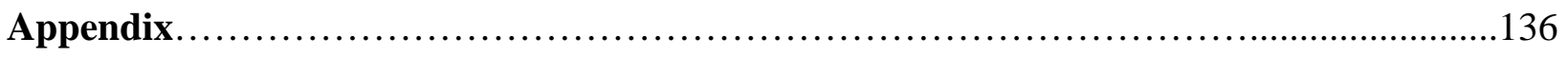




\section{List of Figures}

\section{Chapter 1: Introduction}

Figure 1a. First rotation of four stroke engine (A) Intake stroke (B) Compression stroke........7

Figure 1b. Second rotation of four stroke engine (C) Power stroke (D) Exhaust stroke..........7

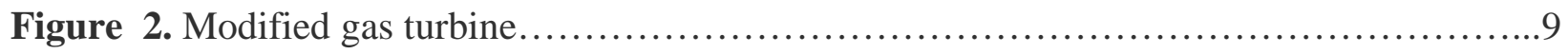

Figure 3. Temperature dependence for the reaction of $\mathrm{OH}$ with benzene...................18

Figure 4. Temperature dependence for the reaction of $\mathrm{OH}$ with propene...................18

Figure 5. The reaction rate of propene as a function of total density of the gas................20

Figure 6. Structures of two, three, and four ringed PAHs.............................23

Figure 7. Proposed scheme of soot formation (adapted from Richter and Howard)............27

\section{Chapter 2: Experimental Methods}

Figure 1. Experimental schematic for the kinetic measurements from 300 to $450 \mathrm{~K}$ and 1 to 10

Torr .43

Figure 2. Lobed rotors in Roots Blower vacuum pump..............................45

Figure 3. Plot of measured flowrate vs read flowrate for the 200 sccm mass flow controller....47

Figure 4. Schematic of laser induced fluorescence of Hydroxyl Radical.....................52

Figure 5. OH LIF spectrum for $A^{2} \Sigma^{+}-X^{2} \Pi$ electronic transition $\left(v^{\prime}=1, v^{\prime \prime}=0\right) \ldots \ldots \ldots \ldots \ldots \ldots . . . .53$

Figure 6. Fluorescence decay due to the dye laser..................................... 
Figure 7. Schematic of a laser cavity

Figure 8 (a) Three-level and (b) Four-level lasing systems. .57

Figure 9. Energy levels in (a) Free $\mathrm{Nd}^{3+}$ and (b) $\mathrm{Nd}^{3+}$ split by crystal field interactions.........59

Figure 10. Nd:YAG laser profile at $1064 \mathrm{~nm}$ 62

Figure 11. Energy level scheme for a dye molecule nine processes important in laser action....63

Figure 12. Structure of Rhodamine 590 dye .64

Figure 13. Reflection mode photocathode in a circular cage in side-on photomultiplier tube....66

Figure 14. Timing between the pump and probe lasers. .68

Figure 15. Emission of $\mathrm{OH}$ fluorescence following dye laser excitation.... .69

Figure 16. A plot of $\mathrm{k}_{1 \mathrm{st}}$ vs number density of propene at 5 Torr. The line is obtained using a linear fit to the data and $\mathrm{k}_{2 \text { nd }}$ values are presented with $2 \sigma$ precision. .72

\section{Chapter 3: Reaction of the OH Radical with Phenylacetylene}

Figure 1. Plot of $\mathrm{OH}$ radical reaction rate coefficients against electrophilic substituent constants for monosubsituted aromatic compounds. The red dot is for phenylacetylene...

Figure 2. Schematic of experimental set up to determine the cross-section of phenylacetylene at $266 \mathrm{~nm}$.

Figure 3. Bubbler pressure vs number density of phenyl acetylene in the reaction cell. The solid line represents the fit relating the bubbler pressure to the number density of phenylacetylene, 
given by the equation. $\mathrm{A}, \mathrm{B}$ and $\mathrm{C}$ are constants from the fit and $\mathrm{P}_{\text {bubb }}$ is the bubbler pressure in Torr

Figure 4. Typical plot of $\ln \frac{\mathrm{I}}{\mathrm{I}_{0}}$ vs Pabscell (Torr) at $254 \mathrm{~nm}$....

Figure 5. Typical plot of $\ln \frac{\mathrm{I}}{\mathrm{I}_{0}}$ vs Pabscell (Torr) at $266 \mathrm{~nm}$

Figure 6. Plot of cross-section of phenylacetylene at $266 \mathrm{~nm}$ vs molar fraction of phenylacetylene in helium.

Figure 7. Schematic of experimental setup used for synthesis of HONO.... .88

Figure 8. Long arm window to prevent the diffusion of phenylacetylene. 90

Figure 9. Typical traces of $\mathrm{OH}$ decays for varying concentrations of Phenyl acetylene at photolysis laser fluence of $147 \mathrm{~mJ} / \mathrm{cm}^{2}$. Plots are generated as a function of decay signal as a function of delay time between the probe laser and photolysis laser, fit to a single exponential function with corresponding residuals and $\mathrm{k}_{1 \mathrm{st}}$ values

Figure 10. Typical traces of $\mathrm{OH}$ decays for varying concentrations of Phenyl acetylene at photolysis laser fluence of $147 \mathrm{~mJ} / \mathrm{cm}^{2}$. Plots are generated as a function of decay signal as a function of delay time between the probe laser and photolysis laser, fit to a single exponential function with corresponding residuals and $\mathrm{k}_{1 \mathrm{st}}$ values

Figure 11. Power dependence of the second order rate constant $\left(k_{2 n d}\right)$ plotted as a function of fluence of photolysis laser at $266 \mathrm{~nm}$. The error bars for individual $\mathrm{k}_{2 \mathrm{nd}}$ values are $2 \sigma$

precision. 
Figure 12. Plot of second order rate constant $\left(\mathrm{k}_{2 \mathrm{nd}}\right)$ a function of laser fluence using HONO as

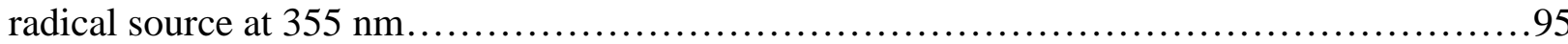

Figure 13. First order rate constants $\left(\mathrm{k}_{1 \mathrm{st}}\right)$ obtained from $\mathrm{OH}$ decay profiles plotted against concentrations of phenyl acetylene. The second order rate constant $\left(\mathrm{k}_{2 n d}\right)$ is the slope of the solid line obtained from a linear least-squares fit with $2 \sigma$ standard deviation .96

Figure 14. Plot of second order rate constant $\left(\mathrm{k}_{2 \mathrm{nd}}\right)$ of the reaction of $\mathrm{OH}$ from HONO photolysis at $355 \mathrm{~nm}$ with phenyl acetylene plotted against pressure of reaction cell. Each data point represents weighted averaged $k_{2 n d}$ value with $2 \sigma$ precision values obtained from multiple runs (minimum 5)

Figure 15. Second order rate constants $\left(\mathrm{k}_{2 n d}\right)$ for the reaction of $\mathrm{OH}$ from $\mathrm{HONO}$ photolysis at $355 \mathrm{~nm}$ with phenyl acetylene plotted against temperature of reaction cell (293 - $423 \mathrm{~K})$. Each data point represents weighted averaged $\mathrm{k}_{2 \mathrm{nd}}$ value with $2 \sigma$ precision values obtained from

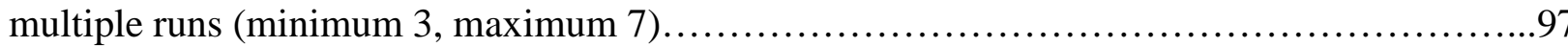

Figure 16. Exothermicites for the addition reaction intermediates and some of their isomers.

\section{Chapter 4: Reaction of the OH Radical with Fulvenallene}

Figure 1. Fulvenallene

Figure 2. Experimental set up for the synthesis of Fulvenallene.

Figure 3. (a) Experimental and (b) simulated ${ }^{1} \mathrm{H}$ NMR spectrum of the liquid fulvenallene dissolved in $\mathrm{CDCl}_{3}$ 
Figure 4. Baseline subtracted IR absorption spectra of the gas mixture recorded with $4 \mathrm{~cm}^{-1}$ resolution.

Figure 5. Baseline subtracted absorption spectrum with $8 \mathrm{~cm}^{-1}$ resolution of the synthesized liquid vapors in helium. The fulvenallene vibrational bands are assigned according to Angell et.

al. 116

Figure 6. (a) Simulated spectrum (B3LYP/CBSB7 method) of fulvenallene and (b) baseline subtracted absorption spectrum of the gas mixture. The calculated spectrum is scaled by a factor 0.952 to match the observed normal modes of fulvenallene 118

Figure 7. Simulated absorption spectra of homophthalic anhydride and benzocyclobutenone using the B3LYP/CBSB7 method. The calculated spectra are scaled by a factor 0.952 . 119

Figure 8. Baseline subtracted absorption spectrum $\left(8 \mathrm{~cm}^{-1}\right.$ resolution) of a gaseous sample, showing additional absorption bands at $1264 \mathrm{~cm}^{-1}, 1037 \mathrm{~cm}^{-1}$ and $820 \mathrm{~cm}^{-1}$ 120

Figure 9 (a) Temporal profile of the $\mathrm{OH}$ radical and (b) pseudo first-order decay as a function of fulvenallene number density recorded at $298 \mathrm{~K}$ and 5 Torr. The red lines are (a) an exponential and (b) a linear fit to the data. The error bars are $2 \sigma$ from the least-squared exponential fits. 124

Figure 10. Second-order rate coefficients $\left(\mathrm{k}_{2 n d}\right)$ as a function of reaction cell temperature from 298 to $450 \mathrm{~K}$. Each data point is averaged $\mathrm{k}_{2 \text { nd }}$ with $1 \sigma$ precision values from multiple runs ( 3 to 5 runs). The red line is the fit to our experimental data and the black dotted line is the abstraction rate constants calculated by da Silva et.al. as a function of temperature (K)................126 


\section{Chapter 5: Conclusion and Future Directions}

Figure 1. Schematic of high temperature pulsed fast flow reactor.......................134 


\section{List of Schemes}

\section{Chapter 1: Introduction}

Scheme 1. Complete combustion of hydrocarbon fuels......................................

Scheme 2. Incomplete combustion of hydrocarbon fuels....................................

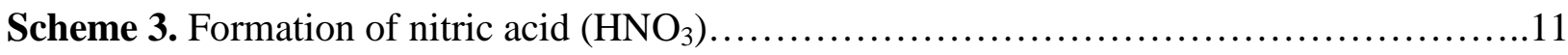

Scheme 4. Formation of sulfurous $\left(\mathrm{H}_{2} \mathrm{SO}_{3}\right)$ and sulfuric $\left(\mathrm{H}_{2} \mathrm{SO}_{4}\right)$ acids.......................11

Scheme 5. Formation of carbon dioxide.............................................. 11

Scheme 6. Hydroxyl radical reacts with methane to yield methyl radical......................15

Scheme 7. Hydroxyl radical reacts with acetaldehyde to form acetyl radical....................16

Scheme 8. Hydroxyl radical reacts with ethylene to form OH-ethylene adduct..................16

Scheme 9. Hydroxyl radical reacts with acetone to form an adduct and acetic acid through $\mathrm{CH}_{3}$

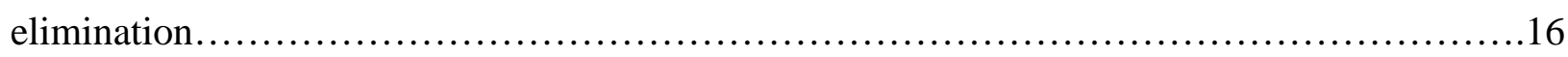

Scheme 10. Hydroxyl radical reacts with benzene to form Van der Waals association

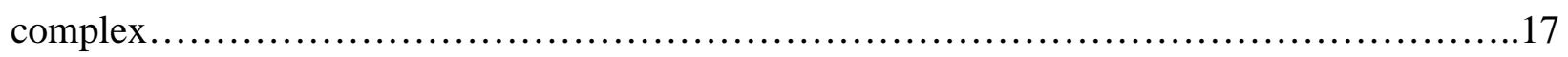

Scheme 11. The reaction of hydroxyl radical with propene..................................19

Scheme 12. Propargyl radical self recombines to form benzene or phenyl radical...............21

Scheme 13. Hydrogen-atom assisted isomerization of fulvene to benzene.....................22

Scheme 14. The reaction of propargyl radical with acetylene to generate benzene..............22 
Scheme 15. Benzene reacts with gaseous hydrogen atom to form phenyl radical....

Scheme 16. The phenyl radical leading to the formation of naphthalene and acenaphthylene. .24

Scheme 17. Formation of naphthalene through the HACA mechanism. .25

Scheme 18. Phenyl radical reacting with benzene to form biphenyl and biphenyl radical reacting

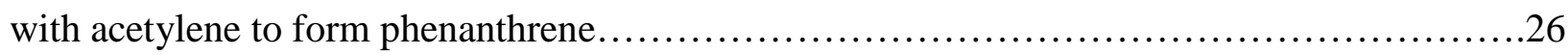

Scheme 19. Hydroxyl radical reacting with phenylacetylene to form phenanthrenyl radical......26

Scheme 20. Resonance structures of fulvenallenyl radical..................................29

Scheme 21. Two propargyl radicals forming 1,5-hexadiyne and 1,2-hexadiene-5-yne...........30

Scheme 22. Two propargyl radicals forming benzene, fulvene, and phenyl radical..............30

Scheme 23. Propargyl radical combines with allyl radical to form benzene via fulvene..........31

Scheme 24. Cross combination of RSRs to form addition products............................31

Scheme 25. Two cyclopentadienyl radicals form naphthalene..............................32

Scheme 26 Two cyclpentadienyl radicals form naphthalene and fulvalene.....................32

Scheme 27. Two cyclopentadienyl radicals react to form naphthalene........................33

Scheme 28. (i) Adduct $\left(\mathrm{C}_{10} \mathrm{H}_{10}\right)$ and (ii) naphthalene and azulene in the study by Knyazev and popov.

Scheme 29. (i) Fulvenalenyl and propargyl form naphthalene (ii) fulvenallenyl and 
cyclopentadienyl form diphenyl and (iii) self-recombination of fulvenallenyl form

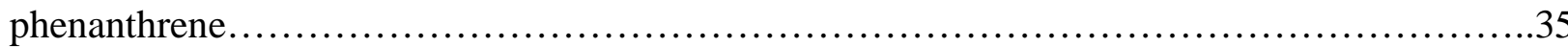

Scheme 30. Resonance structures of $\mathrm{OH}$-phenylacetylene adduct...........................36

\section{Chapter 3: Reaction of the OH Radical with Phenylacetylene}

Scheme 1. Phenyl radical reacts with gaseous acetylene to form phenylacetylene or benzene reacts with ethynyl radical to form phenylacetylene....................................... 80 Scheme 2. Phenylacetylene radical formed from phenylacetylene reacts with gaseous acetylene

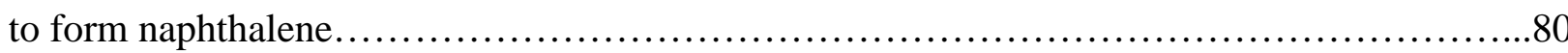

\section{Chapter 4: Reaction of the OH Radical with Fulvenallene}

Scheme 1. Proposed mechanism of fulvenallene formation..................................109 


\section{List of Tables}

\section{Chapter 1: Introduction}

Table 1. Major combustion byproducts as pollutants....................................10

Table 2. Health effect of pollutants from combustion of fossil fuels and biomass adapted from

Smith et.al. and Naeher et.al...........................................................

Table 3. Some PAHs and their fluorescence excitation and emission wavelengths..............23

\section{Chapter 2: Experimental Methods}

Table 1. Excitation and detection wavelengths of the radicals corresponding to their

transitions.

Table 2. Specifications of Nd:YAG laser..........................................62

Table 3. Specifications of tunable dye laser.......................................65

Table 4a. Rate coefficients $\left(\mathrm{cm}^{3} \mathrm{~s}^{-1}\right)$ of the validation reactions compared to published

literature.....................................................................

Table 4b. Rate coefficients $\left(\mathrm{cm}^{3} \mathrm{~s}^{-1}\right)$ for validation of the high temperature setup...............74

\section{Chapter 3: Reaction of the OH Radical with Phenylacetylene}

Table 1. Cross-section of phenylacetylene at $266 \mathrm{~nm}$, molar fraction of phenylacetylene in helium and standard deviation for each set of experiment..............................86

Table 2. Rate coefficients $\left(\mathrm{cm}^{3} \mathrm{~s}^{-1}\right)$ for the $\mathrm{OH}$ reaction with various mono-substituted aromatic rings. .99 
Table 3. Enthalpy of reaction for the abstraction and $\mathrm{H}$-loss channels of $\mathrm{OH}+$

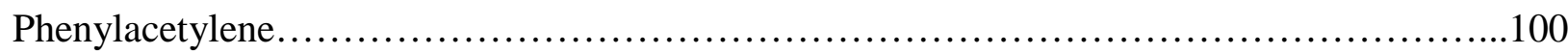

\section{Chapter 4: Reaction of the OH Radical with Fulvenallene}

Table 1. Physical properties of the reactant and products. .109

Table 2. Vibrational assignments of the observed gas phase fulvenallene absorption bands....117

Table 3. Wavenumber, calculated dipole strength, and measured intensity for fulvenallene and homophthalic absorption bands. The ratio of the molar fractions is calculated using equation 2.

Table 4. Rate coefficient and fulvenallene number density for single kinetic measurements, and averages values. Symbols refer to the experiments performed with the same gaseous mixture 125

Table 5. Reaction enthalpy for the abstraction and addition channels of $\mathrm{OH}+$ Fulvenallene calculated using the CBSQB3 method. 


\section{List of Symbols and Abbreviations}

\section{Chapter 1: Introduction}

VOCs

NSOs

FAME

PPO

DI

IDI

SI

CI

ICE

$\mathrm{NO}_{\mathrm{x}}$

$\mathrm{SO}_{\mathrm{x}}$

HCs

PM

IR

PAH

LTC

PPCI

HACA

UV

RSRs

K

PLP-LIF

$\mathrm{Pa}$
Volatile Organic Compounds

Nitrogen, Sulfur, and Oxygen

Fatty Acid Methyl Ester

Pure Plant Oil

Direct Injection

Indirect Injection

Spark Ignition

Compression Ignition

Internal Combustion Engine

Nitrogen Oxides

Sulfur Oxides

Hydrocarbons

Particulate Matter

Infrared

Polycyclic Aromatic Hydrocarbon

Low Temperature Combustion

Partially Premixed Compression Ignition

Hydrogen Abstraction Acetylene Addition

Ultraviolet

Resonance-Stabilized Radicals

Kelvin

Pulsed Laser Photolysis-Laser Induced Fluorescence

Pascal 


\section{Chapter 2: Experimental Methods}

\begin{tabular}{|c|c|}
\hline UHP & Ultra-High Purity \\
\hline CGA & Compressed Gas Association \\
\hline $\mathrm{CF}$ & Conflat \\
\hline iQDP & iQ Dry Dump \\
\hline iQMB & iQ Mechanical Booster \\
\hline $\mathrm{m}^{3} \mathrm{hr}^{-1}$ & Cubic Meter per Hour \\
\hline rpm & Rotations per Minute \\
\hline MFCs & Mass Flow Controllers \\
\hline SSR & Solid State Relays \\
\hline NVOC & Natural Volatile Organic Compound \\
\hline Nd:YAG & Neodymium Yttrium Aluminium Garnet \\
\hline PLP & Pulsed Laser Photolysis \\
\hline LIF & Laser Induced Fluorescence \\
\hline ppm & Parts per Million \\
\hline $\mathrm{nm}$ & Nanometer \\
\hline ns & Nanosecond \\
\hline FWHM & Full Width Half Maximum \\
\hline KBP & Potassium Pentaborate \\
\hline $\mathrm{BBO}$ & $\beta$-barium Borate \\
\hline ps & Picosecond \\
\hline M & Molar \\
\hline PMT & Photomultiplier Tube \\
\hline $\mathrm{mA} / \mathrm{W}$ & Milliamperes/Watts \\
\hline $\mathrm{A} / \mathrm{W}$ & Amperes/Watts \\
\hline
\end{tabular}




\section{Chapter 3: Reaction of the OH Radical with Phenylacetylene}

Sccm

INT

\section{Chapter 4: Reaction of the OH Radical with Fulvenallene}

$\mathrm{kJ}$

Abs

PTFE

NMR

$\mathrm{MHz}$

HSQC

HMBC

1D

2D

NOESY

FTIR

L

Kilojoule
Standard Cubic Meter per Minute

Intermediate
Abstraction
Polytetrafluoroethylene
Nuclear Magnetic Resonance
Megahertz
Heteronuclear Single Quantum Coherence
Heteronuclear Multiband Coherence
One Dimensional
Two Dimensional
Nuclear Overhauser Effect Spectroscopy
Fourier Transform Infrared Spectroscopy
Liter 


\section{Chapter 1: Introduction}

\subsection{Combustion}

During combustion the chemical energy of fuel molecules (solid, liquid, and gas) is converted into usable energy such as heat or work. In the case of complete combustion the reaction of a fuel with an oxidizer in the presence of nitrogen generates carbon dioxide and water along with the production of energy as shown in scheme 1.

Fuel (hydrocarbons) + Air $\left(\mathrm{O}_{2}\right.$ and $\left.\mathrm{N}_{2}\right) \rightarrow \mathrm{CO}_{2}+\mathrm{H}_{2} \mathrm{O}+\mathrm{N}_{2}+$ Energy

Scheme 1. Complete combustion of hydrocarbon fuels

In the case of incomplete combustion, the fuel does not burn completely due to the limited amount of oxygen and therefore generates various products along with the production of energy as shown in scheme 2.

Fuel (hydrocarbons) + Air $\left(\mathrm{O}_{2}\right.$ and $\left.\mathrm{N}_{2}\right) \rightarrow$ unburned hydrocarbons $+\mathrm{NO}_{\mathrm{x}}$ (nitrogen oxides) $+\mathrm{CO}$ + particulates (particulate matters) $+\mathrm{CO}_{2}+\mathrm{H}_{2} \mathrm{O}+$ VOCs (volatile organic compounds-benzene, ethylbenzene, cyclopentane, propene, etc.) + Energy

Scheme 2. Incomplete combustion of hydrocarbon fuels

About $90 \%$ of the worldwide energy is obtained from the combustion of fuel. ${ }^{1}$ In practice, combustion of fuel in internal combustion engines is incomplete, therefore releasing less energy and more undesirable products. The fuel efficiency of modern gasoline engines is

only about $14-30 \% .{ }^{2}$ In order to increase the efficiency of engines and minimize pollutants it is crucial to obtain a full understanding of the chemical reactions governing combustion chemistry. 
This requires investigating the thermochemistry, kinetics, and reaction mechanism of this complex chemical system.

Combustion byproducts, especially soot, have drawn much interest owing to their adverse health effects. Soot formation is poorly understood due to the limitation of accurate experimental reaction kinetics as well as the lack of knowledge about the elementary reaction mechanisms leading to molecular growth. These key parameters contribute to the inaccuracy of combustion models that are used to optimize the efficiency of engines and reduce emissions. In addition, improved combustion, towards complete oxidation of the hydrocarbon fuels will help reducing fuel consumption and achieve clean emissions from combustion devices. ${ }^{3}$

D.P. Mishra ${ }^{1}$ defines fuels as being compounds that can donate electrons and oxidizer as compounds that can accept electrons. In other words, the molecule with higher electronegativity acts as an oxidizer whereas that with the lower electronegativity acts as a fuel. The most important oxidizer is undoubtedly the oxygen present in air.

\subsection{Types of Fuels}

Fuels can be categorized into conventional and non-conventional or alternative resources. ${ }^{4}$

1.2.1 Conventional Resource - Upon consumption, a conventional resource cannot be replenished by natural means. Fossil fuels are the most widely used conventional resources especially for use in transportation. Fossil fuels were formed 300 million years ago. ${ }^{5}$ Organisms, plants, and animals died, decayed, decomposed, and were buried by earth materials for several million years. After several million years, the decomposed material formed organic matter that eventually led to fossil fuels. ${ }^{5}$ 
Fossil fuels are mainly carbon-based fuels such as coal, oil or petroleum, and natural gas. These have been the dominant source of energy providing over $80 \%$ of the total energy for hundreds of years. ${ }^{6,7}$ In this dissertation, only petroleum and natural gas are discussed.

1.2.1.1 Petroleum - Petroleum (crude oil) is the primary source of energy production. ${ }^{8}$ It is a yellow to black viscous liquid containing mainly carbon (82-87\%) and hydrogen (11-15\%) along with other elements such as oxygen $(<1.5 \%)$, nitrogen $(<1.5 \%)$, and sulfur $(<6 \%)$ in small amount. Alkanes are the main constituents of petroleum and contain numerous isomers. ${ }^{9}$ Petroleum comprises of alkanes, cycloalkanes, aromatics, and heteroatomic compounds containing nitrogen, sulfur, and oxygen (NSOs).

Alkanes may be straight chained or branched. The number of carbon atoms ranges from C1 to C78. Alkenes and alkynes are not found initially but they result from various refinery processes. Cycloalkanes are mostly found in the form of cyclopropane $\left(\mathrm{C}_{3} \mathrm{H}_{6}\right)$, cyclobutane $\left(\mathrm{C}_{4} \mathrm{H}_{8}\right)$, cyclopentane $\left(\mathrm{C}_{5} \mathrm{H}_{10}\right)$, and cyclohexane $\left(\mathrm{C}_{6} \mathrm{H}_{12}\right)$. Aromatic compounds in petroleum vary from 10 to 50\%. Aromatics include single ring compounds e.g. benzene, toluene, xylene and its isomers, and mesitylene (1,3,5-trimethylbenzene) and condensed ring forms e.g., naphthalene, anthracene, phenanthrene, pyrene, etc. referred to as polycyclic aromatic hydrocarbons. ${ }^{9}$

One of the important fuels obtained from petroleum during the distillation of oil is diesel. Diesel is cheaper but lower quality than gasoline (the first distillation product of oil). The molecules in diesel fuels are larger e.g., alkylated naphthalenes and n-alkanes are desirable e.g., hexadecane $\left(\mathrm{C}_{16} \mathrm{H}_{34}\right)$. High boiling components such as polycyclic aromatic hydrocarbons are undesirable products. Biodiesels made from vegetable oils, animal fats or grease wastes are 
known to be less pollutant. The elements present in biodiesel are C (76\%), H (13\%), O (11\%), N $(0 \%)$, and S $(0 \%)^{9}$

The quality of diesel fuel is measured by the cetane number. A high cetane number corresponds to faster ignition of the fuel. For the same number of carbons the cetane number ranks according to n-alkanes>alkenes>cycloalkanes>alkylaromatic. For alkanes, the cetane number drops as the number of carbons decreases. Diesel is more efficient than gasoline and is used specifically in diesel engines. Diesel engines are a type of internal combustion engine that is used in cars, trucks, busses, trains, boats, and agricultural vehicles. Marine ships use heavier diesel engines than those used for automobiles and trucks.

1.2.1.2 Natural Gas - The elements present in natural gas are C (76\%), H (18\%), O $(<2 \%), N(<5 \%)$, and S $(<1 \%)$ with methane as the main constituents. Other alkanes such as ethane, propane, butane, and pentane are present as well as nitrogen, carbon dioxide, hydrogen sulfide, and helium. ${ }^{10}$ It is used in water heaters, cooking stoves, gas turbines and some engines. It is also used to produce hydrogen, ammonia, sulfur, carbon black, and ethylene (another petrochemical product). ${ }^{10}$

Natural gas is found under pressure in rock reservoirs. It is formed by the degradation of organic materials accumulated for millions of years. Natural gas can be formed at shallow depths by the anaerobic decomposition of sedimentary organic material at low temperature. It can also be formed at deeper depths by thermal cracking of sedimentary organic material into hydrocarbon liquid and gas (oil in conjunction with gas-primary gas) and thermal cracking of oil at high temperatures (secondary gas). ${ }^{10}$ 
1.2.2 Non-Conventional Resource - Non-conventional sources can be replenished quickly and energy obtained from these sources is believed to be cleaner compared to fossil fuel energy. Biofuels are an example of alternative fuels.

1.2.2.1 Biofuels and its Types - Biofuels are liquid (e.g., methanol, ethanol, and biodiesel) or gaseous (methane and hydrogen) fuels derived from biomass. Biomass is an organic material obtained from living organisms especially plants or plant based materials e.g., trees, grasses, agricultural crops that are not used as food. ${ }^{11}$ The sources of biomass are also called feedstocks. Some examples of feedstocks are oilseed crops, sawdust, black liquor (byproduct of paper making process), cheese whey (byproduct of cheese making process), vegetable oils from plant sources for biodiesel, and animal manure, fats, oil, greases, unused wood from construction, packaging wastes, solid wastes, and landfill gases. ${ }^{11}$ Based on feedstock and utilization, biofuels can be divided into two categories: First generation and second generation. ${ }^{12}$

(i) First Generation - These biofuels are derived from materials such as sugar, starch, esters of glycerol, free fatty acids and glycerides (fats and vegetable oils), and fatty acids, cellulose, hemicelluloses, and lignin (wood for solid biofuels). Some of the first generation biofuels are bioalcohols (e.g., methanol, ethanol, propanol, butanol), fatty acid methyl ester (FAME or biodiesel) and pure plant oil (PPO).

(ii) Second Generation - These are derived by utilizing unused land and consuming wastes. Feedstocks e.g., lignocellulosic material from byproducts of agriculture such as rice husk, sawdust, corn rub etc. are used for the second generation biofuels. ${ }^{13}$ High organic contents in wastes possess high amount of carbohydrates and protein. Anaerobic digestion of wastes makes it suitable for bioenergy production. ${ }^{14}$ 
The most common biofuel is ethanol ${ }^{15}$ and the key feedstocks to produce ethanol are corn, sugarcane, wheat, sugar beet, soybean, palm oil. ${ }^{16}$ The main advantage of using ethanol is its economic value and feasibility to produce in high yield.

\subsection{Types of Combustion Engines}

Combustion engines are devices that use heat and pressure released by the combustion chemical reaction to generate mechanical energy that can be used to run automobiles or industrial machines. In internal combustion engines the chemical energy from the chemical reaction is directly utilized to do mechanical work. ${ }^{3,17}$ Here, internal combustion engines are briefly discussed as they are dominant in vehicles.

The reciprocating piston-cylinder configuration is the most popular internal combustion engine with the aim of achieving high work output with a high efficiency. In this configuration, a piston moves back and forth in a cylinder transmitting power through the rod. Flow of gas into and out of the engine is controlled using valves or ports. This reciprocating engine can be designed to operate in four or two strokes. ${ }^{18}$ In this dissertation only four-stroke engine will be discussed as they are the most efficient. Four-stroke engine completes its one cycle in four steps. Figure 1a shows the intake and compression strokes of four stroke engine.

(A) Intake stroke - During this stroke the inlet valve is open and the piston moves down the cylinder. Air or premixed fuel is sucked in. The exhaust valve remains closed.

(B) Compression stroke - During this stroke both valves remain closed and the piston moves upward compressing the mixture of air and fuel. As the piston reaches almost the top part, ignition occurs. 


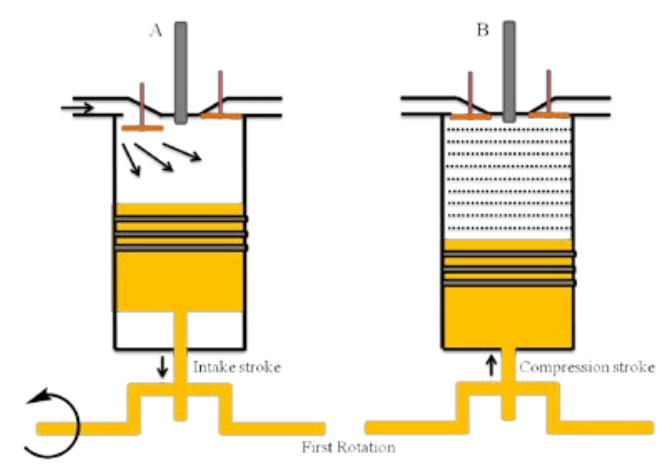

Figure 1a. First rotation of four stroke engine (A) Intake stroke (B) Compression stroke ${ }^{19}$ Figure 1b shows the power and exhaust strokes that occur in four-stroke engine

(C) Power stroke - Both intake and exhaust valves are closed. The combustion flame propagates throughout the space of the cylinder raising the pressure and temperature. The piston is forced down. At the end of power stroke, the exhaust valve opens.

(D) Exhaust stroke - Exhaust valve remains open and the piston moves upward forcing the emission of remaining gasses and particulate matters. At its end the exhaust valve closes.

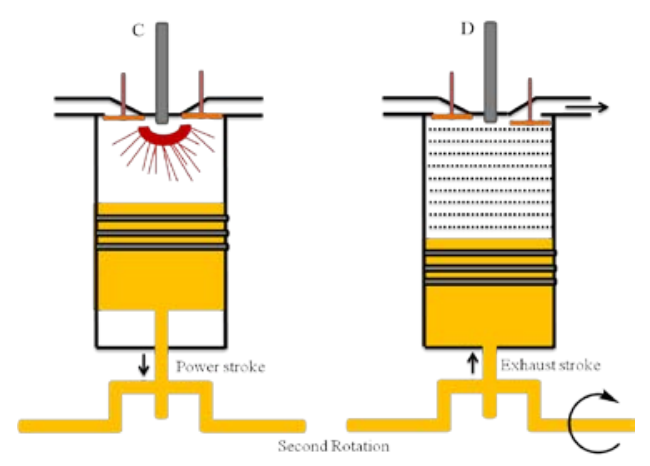

Figure 1b. Second rotation of four stroke engine (C) Power stroke (D) Exhaust stroke ${ }^{19}$ These two cycles in the four stroke engine are repeated rapidly during the operation.

The two main types of four stroke internal combustion engines are: ${ }^{18}$

(i) Spark Ignition (SI) or Otto Engine - The fuel is ignited by a spark and the engine completes the cycle as described in four stroke engine.

When the engine is working, controlled air enters through the throttle and is evenly gets distributed in all the cylinders as the piston moves back. The fuel is injected through the intake 
port or valve and the fuel air are mixed homogeneously in the cylinder. The homogeneous mixture is then compressed by the inward movement of the piston. The spark ignites the mixture raising its pressure and temperature. The flame extinguishes as it reaches the walls of the cylinder. The huge pressure developed forces the piston back again due to the expansion of the gaseous mixture. At the end, the exhaust gaseous mixture is expelled through the exhaust valve.

(ii) Compression Ignition (CI) or Diesel Engine - In the diesel engine, a high rise in temperature and pressure due to high compression spontaneously ignites the fuel-air mixture. During the compression stroke, air is compressed beyond the autoignition temperature of the fuel. Then the diesel spray evaporates, mixes, ignites, and burns. ${ }^{17}$

Diesel fuel can be burned in the compression engine by direct injection (DI) method or indirect injection (IDI) method. ${ }^{17}$ In the indirect injection method, air is compressed in the prereaction chamber during the compression stroke. This creates a turbulent flow and the sprayed diesel mixes well. An increase in pressure in the pre-reaction chamber that is greater than that in the main chamber initiates the ignition of the fuel-air mixture. The flame makes its way to the main chamber resulting in a swirling turbulent flame. Because automobiles operate over a wide range of speeds, the indirect injection method is used whereas in ships, trucks, and electric power generation, the direct injection method is used.

Diesel engines have higher compression ratios than spark ignition engine. This results in better fuel-air mixing ratio and ignition that leads to higher power and better efficiency. The knock is lower than in SI engine because of fuel mixing prior to ignition. Knock is the pinging sound produced inside the engine due to incorrect fuel-air mixture. Still, because of reduced mixing time compared to SI engine, the combustion is incomplete leading to decreased power. ${ }^{3}$ 
Besides internal combustion engines, there are other methods for power generation such as wind turbines, water turbines, steam turbines and gas turbines.

(iii) Gas Turbines - Turbines operate through spinning motion. Gas turbines are the most popular turbines and are based on the same principle as internal combustion engines. They have high efficiency (nearly $500 \mathrm{~kW}$ more than internal combustion engine, ICE) and power per weight. These gas turbines are used to power aircrafts, trains, ships, electric generators, etc.

A Gas turbine consists of a compressor-burner-turbine combination. It consists of a primary zone for combustion that requires compressed air (20-30\%). This region has a high pressure and temperature. The secondary zone is mainly for the exhaustion of the remaining gasses and byproducts. Basically, a fuel-air mixture is compressed in the primary zone and then ignited to produce high pressure. The gas is then expanded to make the turbine move. Spinning of turbine makes the propeller move or spin the shaft in vehicular application and is used for power generation. Figure 2 shows the simple schematic of a modified version of a gas turbine. A diffuser (not shown in the figure) is added to reduce the air inlet velocity that makes primary and secondary air section. A flame holder is inserted to allow enough reaction zone and for stabilization. The geometry is conical in inlet and outlet for uniform gas discharge temperature. Fuel nozzle is also designed to provide conical spray of fuel droplets. ${ }^{3}$

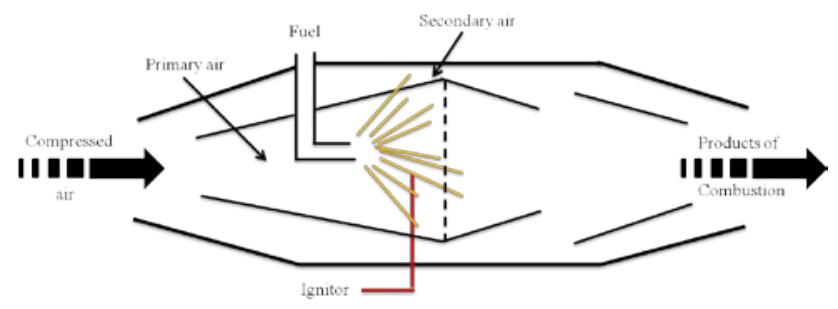

Figure 2. Modified gas turbine ${ }^{3}$ 


\subsection{Combustion Byproducts}

Fuels burn completely or incompletely and generate products along with the production of energy. In internal combustion engines, the fuel does not burn completely and various products are generated. These unwanted byproducts result mainly from the combustion of fossil fuels and biofuels ${ }^{20}$ and are considered as the primary pollutants. The most important pollutants are summarized in table 1 .

Table 1. Major combustion byproducts as pollutants

\begin{tabular}{|c|c|c|c|c|}
\hline Pollutants & $\begin{array}{l}\text { Nitrogen Oxides } \\
\left(\mathrm{NO}_{\mathrm{x}}\right)^{21}\end{array}$ & $\begin{array}{l}\text { Carbon Monoxide } \\
\text { (CO) }\end{array}$ & $\begin{array}{l}\text { Hydrocarbons } \\
\text { (HCs) }\end{array}$ & $\begin{array}{l}\text { Particulate Matter } \\
(\mathrm{PM})^{22}\end{array}$ \\
\hline Composition & $\begin{array}{l}\text { Mainly comprise } \mathrm{NO} \\
\text { and } \mathrm{NO}_{2}\end{array}$ & -- & $\begin{array}{l}\text { mainly } \\
\text { formaldehyde, } \\
\text { acetaldehyde, 1,3- } \\
\text { butadiene, and } \\
\text { benzene }\end{array}$ & $\begin{array}{l}\text { Mixture of solid } \\
\text { carbon material or } \\
\text { soot and organic } \\
\text { materials consisting } \\
\text { of hydrocarbons } \\
\text { and their partial } \\
\text { oxidation products } \\
\text { e.g. polycyclic } \\
\text { aromatic } \\
\text { hydrocarbons. } \\
\text { smoke, soot }\end{array}$ \\
\hline Characteristic & $\begin{array}{l}\mathrm{NO} \text { is colorless and } \\
\mathrm{NO}_{2} \text { is brown gas. } \\
\text { Both are toxic and } \\
\text { play a role in } \\
\text { producing } \\
\text { photochemical smog }\end{array}$ & $\begin{array}{l}\text { Colorless, odorless } \\
\text { gas, toxic, even fatal } \\
\text { in high } \\
\text { concentration, } \\
\text { bonds with } \\
\text { hemoglobin } \\
\text { interfering with } \\
\begin{array}{l}\text { oxygen transport, } \\
\text { oxidizes to } \mathrm{CO}_{2}\end{array}\end{array}$ & $\begin{array}{l}\text { Hydrophobic, } \\
\text { highly flammable, } \\
\text { toxic }\end{array}$ & $\begin{array}{l}\text { Fine }(<2.5 \mu \mathrm{m} \text { in } \\
\text { size }) \text { rather than } \\
\text { coarse }(10-2.5 \\
\mu \mathrm{m}),{ }^{17} \text { toxic }\end{array}$ \\
\hline Origin & $\begin{array}{l}\text { Nitrogen and oxygen } \\
\text { dissociate into their } \\
\text { atomic forms and } \\
\mathrm{NO}_{\mathrm{x}} \text { are formed. } \\
\mathrm{NO} \text { oxidizes to } \mathrm{NO}_{2} \text {. } \\
\text { An example of } \mathrm{NO} \\
\text { and } \mathrm{NO}_{2} \text { formation. } \\
\mathrm{O}+\mathrm{N}_{2} \leftrightarrow \mathrm{NO}+\mathrm{N} \\
\mathrm{NO}+\mathrm{O} \leftrightarrow \mathrm{NO}_{2}\end{array}$ & $\begin{array}{l}\text { During engine warm } \\
\text { up, limited } \\
\text { evaporation of fuel } \\
\text { leads to a greater } \\
\mathrm{CO} \text { emission. }{ }^{24} \\
\text { Another source is } \\
\text { the decomposition } \\
\text { of } \mathrm{CO}_{2} \text { at high } \\
\text { temperatures. }\end{array}$ & $\begin{array}{l}\text { Unburned } \\
\text { hydrocarbon fuels } \\
\text { emitted during the } \\
\text { start-up and } \\
\text { warming stage } \\
\text { due to limited } \\
\text { evaporation and } \\
\text { oxidation of the } \\
\text { fuel. }\end{array}$ & $\begin{array}{l}\text { Initiates from the } \\
\text { reaction of small } \\
\text { radicals, molecules } \\
\text { with fuel during } \\
\text { combustion }\end{array}$ \\
\hline
\end{tabular}


$\mathrm{NO}_{\mathrm{x}}$ and $\mathrm{SO}_{\mathrm{x}}$ affect the environment by forming acid rains as shown in scheme 3 and 4 respectively.

$$
\mathrm{NO}_{2}+\mathrm{OH} \text { (from water vapor) }+\mathrm{h} v \rightarrow \mathrm{HNO}_{3}
$$

Scheme 3. Formation of nitric acid $\left(\mathrm{HNO}_{3}\right)^{21}$

$$
\mathrm{SO}_{2}+\mathrm{H}_{2} \mathrm{O} \rightarrow \mathrm{H}_{2} \mathrm{SO}_{3} \text { and } \mathrm{SO}_{3}+\mathrm{H}_{2} \mathrm{O} \rightarrow \mathrm{H}_{2} \mathrm{SO}_{4}
$$

Scheme 4. Formation of sulfurous $\left(\mathrm{H}_{2} \mathrm{SO}_{3}\right)$ and sulfuric $\left(\mathrm{H}_{2} \mathrm{SO}_{4}\right)$ acids ${ }^{21}$

These $\mathrm{NO}_{\mathrm{x}}$ are also responsible for increasing the ground level ozone concentration, which is a component of photochemical smog (mixture of nitrogen oxides, ground level ozone, volatile organic compounds, etc.). These acid rain and smog damage forests, streams and agricultural products.

$\mathrm{CO}$ is produced mostly in fuel rich combustion and contributes to smog production by reacting with oxygen and nitrogen in the exhaust stream and atmosphere. It also contributes to increasing the concentration of $\mathrm{CO}_{2}$ by reacting with $\mathrm{O}_{2}$ and $\mathrm{NO}$ as shown in scheme $5 .{ }^{17}$ Excess $\mathrm{CO}_{2}$ produced in the atmosphere absorbs infrared (IR) light escaping from the Earth's atmosphere and contributes to Earth greenhouse effect.

$$
\mathrm{CO}+1 / 2 \mathrm{O}_{2} \rightarrow \mathrm{CO}_{2} \text { and } \mathrm{NO}+\mathrm{CO} \rightarrow 1 / 2 \mathrm{~N}_{2}+\mathrm{CO}_{2}
$$

Scheme 5. Formation of carbon dioxide

Exposure to the emissions from combustion has several health effects to humans. These pollutants affect respiration, skin, eyes, nose, lungs, liver, kidneys, and stomach. Long exposure can even lead to death. For example, CO has 200 times more affinity to hemoglobin than 
oxygen. When $\mathrm{CO}$ is inhaled $\mathrm{CO}$ binds with hemoglobin to form carboxyhemoglobin making the tissues oxygen deficient. CO poisoning is the most common fatal air poisoning. The health effects of combustion emissions are summarized in table 2.

Table 2. Health effect of pollutants from combustion of fossil fuels and biomass adapted from Smith et.al. ${ }^{25}$ and Naeher et.al. ${ }^{26}$

\begin{tabular}{|c|c|}
\hline Pollutant & Health effect \\
\hline Nitrogen oxides $\left(\mathrm{NO}_{\mathrm{x}}\right)$ & $\begin{array}{l}\text { Respiratory problem (bronchial reactivity), increased susceptibility to } \\
\text { bacterial and viral lung infections }\end{array}$ \\
\hline Carbon monoxide (CO) & $\begin{array}{l}\text { Reduced oxygen delivery to tissues owing to formation of } \\
\text { carboxyhemoglobin, can be acutely fatal }\end{array}$ \\
\hline Sulfur oxides $\left(\mathrm{SO}_{\mathrm{x}}\right)$ & Respiratory problems (bronchial reactivity), reduces nose sensitivity \\
\hline Particulate matter and soot & $\begin{array}{l}\text { Eye irritation, Bronchial irritation, inflammation, increased reactivity, } \\
\text { increased cardiovascular mortality }\end{array}$ \\
\hline $\begin{array}{l}\text { Organic air pollutants: } \\
\text { Benzene } \\
\text { Acetaldehyde } \\
\text { Phenols } \\
\text { Formaldehyde, } \\
\text { butadiene, Cresols } \\
\text { PAHs (Pyrene, Benzopyrene } \\
\text { Benzo(a)pyrene } \\
\text { Dibenzopyrenes, } \\
\text { Dibenzocarbazoles) }\end{array}$ & $\begin{array}{l}\text { Respiratory problems } \\
\text { Carcinogenicity } \\
\text { Co-carcinogenicity } \\
\text { Mucus coagulation, cilia toxicity } \\
\text { Short term effects-eye and skin irritation }{ }^{27} \text {, nausea }{ }^{27} \text {, vomiting and } \\
\text { diarrhea (high concentration) }{ }^{27} \text {, Inflammation } \\
\text { Long term effects-Skin, lung, bladder, and gastrointestinal cancers, } \\
\text { DNA, cataracts, liver and kidney damage } \\
\text { Gene mutation cell damaging and cardiopulmonary mortality } \\
\text { Asthma }{ }^{28} \text {, Increased allergic sensitization }\end{array}$ \\
\hline
\end{tabular}

\subsection{Low Temperature Combustion (LTC): A Way to Improve Combustion Devices}

Extensive research has focused on low temperature combustion that could mitigate pollutants and alleviate efficiency of engines. LTC combines the advantages of both spark ignition and diesel compression engines by using auto ignition in lean and homogeneous fuel-air mixture. ${ }^{17}$ Spark ignition engines operate at low compression ratio with increased pumping loss whereas diesel engines have high compression ratio and higher efficiency. ${ }^{29}$ In LTC, as no spark plug is required, it is possible to inject very low fuel concentrations and maintain low 
temperatures $(<2000 \mathrm{~K})$ throughout the entire combustion process. Overall this leads to fuel economy and reduced $\mathrm{NO}_{\mathrm{x}}$ and carbon emissions. ${ }^{29}$.

In LTC, after autoignition, the flame propagates as well as ignites at various spots in the chamber and the combustion duration is shorter while still releasing more energy. Because of homogeneous mixture, initiation of ignition is kinetically controlled rather than diffusion controlled as in non-premixed mixture. ${ }^{17}$ So cylinder temperature, pressure, and fuel composition play important role in initiating the ignition. Use of partially premixed lean fuel-air mixture, often referred as partially premixed compression ignition (PPCI), reduces the amount of soot in the exhaust by increasing the combustion duration. ${ }^{30}$ This also reduces the high-pressure rise allowing partial mixing of fuel inside the cylinder.

Under very lean fuel conditions, incomplete combustion at temperatures lower than 1500 K still lead to the formation of large hydrocarbons. ${ }^{31,32}$ The development of low-temperature technologies is therefore partially limited by the lack of understanding of molecular growth and especially PAH formation. One other advantage of LTC is that it can be operated with liquid and gaseous fuels.

\subsection{Combustion Chemistry}

Combustion chemistry is initiated by the reaction of abundant fuel molecules with air $\left(\mathrm{O}_{2} / \mathrm{N}_{2}\right)$ at high temperatures and pyrolyzes to give reactive intermediate species. These intermediate species, mostly small radicals and atoms, are responsible for propagating the chemical reactions leading to molecular growth. During this complex chemical transformation, molecular bonds are broken and new bonds are formed resulting in heat release that can be used for mechanical works. Developing a fundamental understanding of the chemistry of these 
intermediates is vital in order to develop new combustion strategies with lower pollutant emission and higher efficiency.

\subsection{Important Species in Combustion}

The most important reactive intermediates in fuel chemistry are radicals. They are odd electron species resulted from the homolytic cleavage process initiated by heat or chemical reaction with a reagent. Radicals are electrically neutral and highly reactive. There are hundreds of chemical species and hundreds/thousands of chemical reactions that arise from these species in combustion environments. Small radicals such as hydroxyl $(\mathrm{OH})^{33-35}$, methylidene $(\mathrm{CH})^{33,34,36}$, cyano $(\mathrm{CN})^{36,37}$, propargyl $\left(\mathrm{C}_{3} \mathrm{H}_{3}\right)^{38,39}$, and allyl $\left(\mathrm{C}_{3} \mathrm{H}_{5}\right)^{40}$ have been observed to be the key species in combustion engines and flame. Wang and Frenklach ${ }^{41}$ and Marinov et.al. ${ }^{42}$ have included several hundreds of radical reactions in their combustion model. Similarly, Norinaga et.al. ${ }^{43}$ have included 227 species and 827 reactions in their chemical scheme over the 1073-1300 K temperature range.

These radicals are responsible for the formation of key hydrocarbons in combustion, and especially aromatic species. Benzene is present in flames ${ }^{17,44,45}$ and is widely accepted to be formed by the self-recombination of propargyl radicals. ${ }^{46-48}$ The recombination of propargyl could also form phenyl or $\mathrm{C}_{6} \mathrm{H}_{6}$ isomers. ${ }^{48}$ The reaction of benzene with $\mathrm{C}_{2} \mathrm{H}$ radical may form phenylacetylene through an addition/H-loss mechanism. ${ }^{49,50}$ According to the hydrogen abstraction acetylene addition (HACA) mechanism (described in section 1.10.1), benzene may further react with atomic hydrogen to give the phenyl radical $\left(\mathrm{C}_{6} \mathrm{H}_{5}\right)$ and molecular hydrogen. ${ }^{41,51,52}$ The reaction of phenyl radical with acetylene followed by H-loss leads to the formation of phenylacetylene $\left(\mathrm{C}_{6} \mathrm{H}_{5} \mathrm{CCH}\right)$. 
Larger radicals such as fulvenallenyl $\left(\mathrm{C}_{7} \mathrm{H}_{5}\right)^{53}$ and cyclopentadienyl $\left(\mathrm{C}_{5} \mathrm{H}_{5}\right)^{54,55}$ have also recently been proposed as important intermediates in combustion. Theoretical investigations predict that the fulvenallenyl radical may be formed by hydrogen abstraction from ${ }^{53}$ or unimolecular decomposition of fulvenallene $\left.\left(\mathrm{C}_{7} \mathrm{H}_{6}\right)\right)^{56,57}$ which is the main toluene $\left(\mathrm{C}_{6} \mathrm{H}_{5} \mathrm{CH}_{3}\right)^{56,58-60}$, benzyl $\left(\mathrm{C}_{6} \mathrm{H}_{5} \mathrm{CH}_{2}\right)^{61-64}$ pyrolysis and photolysis products. ${ }^{65}$ This dissertation focuses on the reaction of hydroxyl radical $(\mathrm{OH})$ with phenylacetylene $\left(\mathrm{C}_{8} \mathrm{H}_{6}\right)$ and fulvenallene $\left(\mathrm{C}_{7} \mathrm{H}_{6}\right)$.

\subsection{OH Radical Reaction Scheme}

The $\mathrm{OH}$ is a key radical in combustion, as it contributes to the oxidation of fuel molecules as well as to the propagation of the radical reaction schemes. The reaction mechanisms characteristic of the hydroxyl radical are (i) abstraction, (ii) addition, and (iii) association.

(i) Abstraction mechanism ${ }^{66-68}$ - This is the most common mechanism of the hydroxyl radical. $\mathrm{OH}$ abstracts a hydrogen atom from the organic compound to form a water molecule and a new radical. In scheme 6 the hydroxyl radical abstracts a hydrogen atom from methane (alkane) to generate a methyl (alkyl) radical and a water molecule. ${ }^{69,70}$

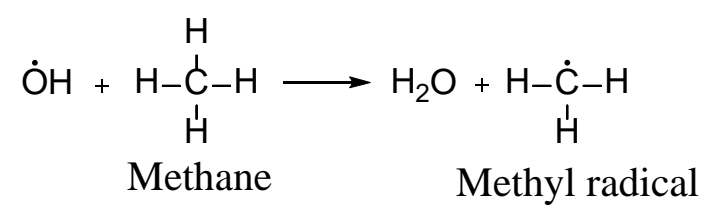

Scheme 6. Hydroxyl radical reacts with methane to yield methyl radical

Similar reaction mechanisms have been observed by abstraction of the $\mathrm{H}$-atom from $\mathrm{C}-\mathrm{H}$ of alkyl part of alcohol e.g. $\mathrm{CH}_{3^{-}},-\mathrm{CH}_{2^{-}}$, $>\mathrm{C}-\mathrm{H}$ groups or from -OH group. ${ }^{67}$ Similarly, it reacts 
with aldehydes and ketones, mostly by abstracting hydrogen atom from $-\mathrm{C}(\mathrm{O}) \mathrm{H}$ or $-\mathrm{C}(\mathrm{O}) \mathrm{R}$ to form water and new radical as shown in scheme $7 .^{71}$

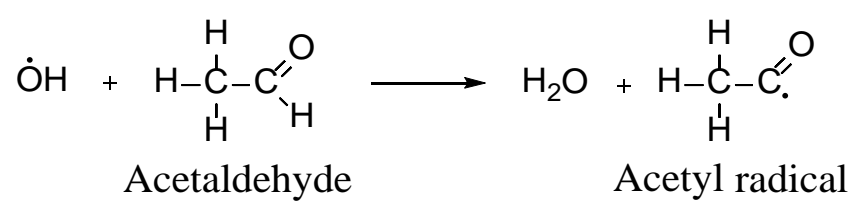

Scheme 7. Hydroxyl radical reacts with acetaldehyde to form acetyl radical

(ii) Addition mechanism ${ }^{66-68}$-The $\mathrm{OH}$ radical can also add to unsaturated carbon to form an adduct.

In scheme 8 hydroxyl radical reacts with ethylene to form an adduct. ${ }^{72,73}$

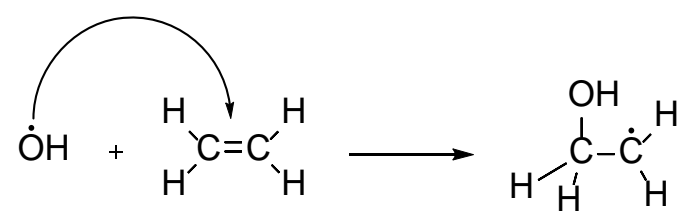

Scheme 8. Hydroxyl radical reacts with ethylene to form OH-ethylene adduct

In the case of acetone as shown in scheme 9, studies suggest that the reaction proceeds via addition ${ }^{74}$ to form acetic acid through $\mathrm{CH}_{3}$ elimination.

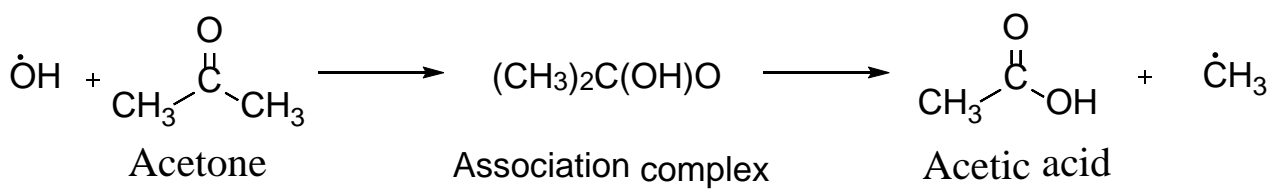

Scheme 9. Hydroxyl radical reacts with acetone to form an adduct and acetic acid through $\mathrm{CH}_{3}$ elimination 
(iii) Association mechanism - In this case the reaction proceeds through the formation of a pre-reactive complex or Van der Waal's complex between the $\mathrm{OH}$ and the unsaturated hydrocarbon. In scheme 10, the $\mathrm{OH}$ radical associates with the benzene ring. ${ }^{75}$

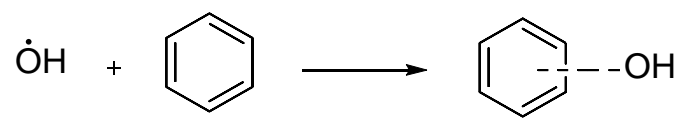

Scheme 10. Hydroxyl radical reacts with benzene to form Van der Waals association complex

\subsubsection{The Temperature Dependence of the Different Reaction Channels}

The reaction rate coefficients for the abstraction, addition, and association channels are dependent on temperature. The abstraction channel often proceeds through a transition state above the energy of the reactants. This results in a reaction rate coefficient that increases with increasing temperature, following the Arrhenius equation. Figure 3 shows the temperature dependence of the $\mathrm{OH}+$ benzene reaction rate coefficient measured experimentally by Tully et.al. ${ }^{75}$ The reaction rate coefficient increases with increasing temperature above $500 \mathrm{~K}$ likely due to increasing contribution of the abstraction of $\mathrm{H}$ atom from benzene by the $\mathrm{OH}$ radical. 


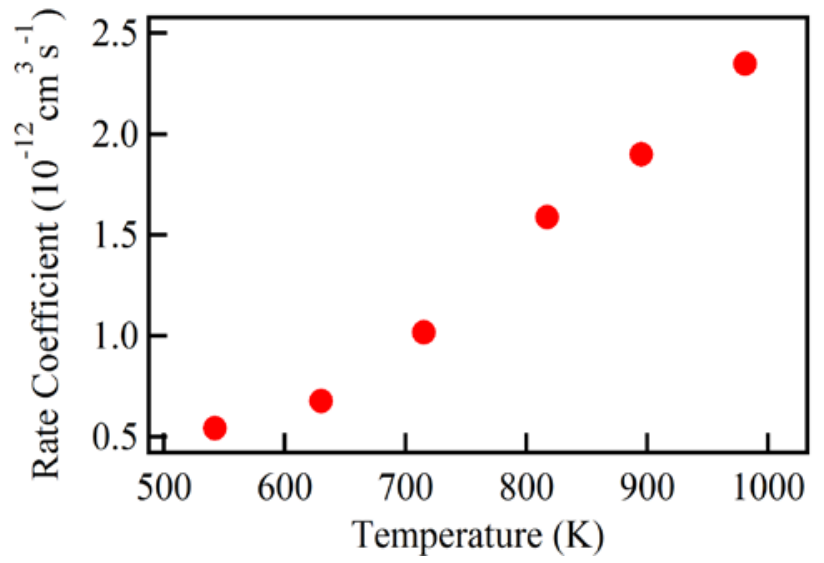

Figure 3. Temperature dependence for the reaction of $\mathrm{OH}$ with benzene. ${ }^{75}$

For addition mechanisms, often the reaction rate coefficient decreases with increase in temperature due to the formation and decomposition of a barrierless activated complex at the entrance channel. Figure 4 shows the temperature dependence of the reaction rate coefficient of $\mathrm{OH}+$ propene investigated experimentally by Vakhtin et.al. ${ }^{72}$ The reaction rate decreases with increase in temperature due to the formation of $\mathrm{OH}$-propene adduct which dissociates back to the reactants at higher temperature.

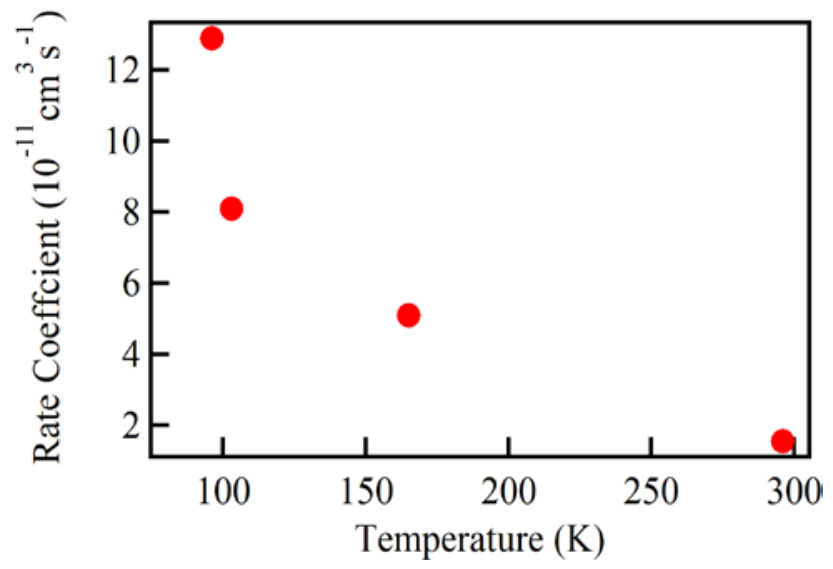

Figure 4. Temperature dependence for the reaction of $\mathrm{OH}$ with propene. ${ }^{72}$ 


\subsubsection{The Effect of Pressure on the Stabilization of the Adduct.}

In scheme 11 the hydroxyl radical reacts with propene to form an energy-rich association adduct. $^{72}$ This adduct has three pathways:

i. It can decompose back to the reactants

ii. It can form products

iii. It can form stabilized adduct by colliding with buffer gas.

$$
\begin{aligned}
& \mathrm{OH}+\mathrm{C}_{3} \mathrm{H}_{6} \stackrel{\mathrm{k}_{+\mathrm{OH}}}{\underset{\mathrm{k}_{-\mathrm{OH}}}{\rightleftharpoons}}\left|\mathrm{C}_{3} \mathrm{H}_{6} \mathrm{OH}\right|^{\ddagger} \stackrel{\mathrm{k}_{\mathrm{i}}}{\longrightarrow} \begin{array}{l}
\mathrm{C}_{3} \mathrm{H}_{6} \mathrm{O}+\mathrm{H} \\
\mathrm{C}_{3} \mathrm{H}_{5}+\mathrm{H}_{2} \mathrm{O}
\end{array} \\
& \mathrm{k}_{4} \downarrow \mathrm{M} \\
& \mathrm{C}_{3} \mathrm{H}_{7} \mathrm{O}
\end{aligned}
$$

Scheme 11. The reaction of hydroxyl radical with propene ${ }^{72}$

Applying steady-state approximation and pseudo-first order approximation, the $\mathrm{k}_{\text {measured }}$ of scheme 11 is given by equation 1 . During the experiment, the value of $k_{\text {measured }}$ is obtained.

$$
\mathrm{k}_{\text {measured }}=\frac{\sum_{\mathrm{i}=1} \mathrm{k}_{\mathrm{i}}+\mathrm{k}_{4}[\mathrm{M}]}{\mathrm{k}_{-\mathrm{OH}}+\sum_{\mathrm{i}=1} \mathrm{k}_{\mathrm{i}}+\mathrm{k}_{4}[\mathrm{M}]} \cdot \mathrm{k}_{+\mathrm{OH}}
$$

For ideal gas

$$
[\mathrm{M}]=\frac{\mathrm{P}}{\mathrm{K}_{\mathrm{B}} \mathrm{T}}
$$

Where $[M]$ is the concentration of buffer gas, $P$ is the pressure of the reaction, $K_{B}$ the Boltzmann constant, and $\mathrm{T}$ the temperature in Kelvin. 
Since the concentration of the buffer gas is proportional to the pressure, $\mathrm{k}_{\text {measured }}$ is related to the pressure. At low pressure, a large fraction of the adduct decomposes back to the reactants reforming $\mathrm{OH}$ and leading to a lower apparent rate coefficient. As the pressure increases more adduct molecules are stabilized leading to less $\mathrm{OH}$ formation and an increasing apparent rate coefficient. At high pressure, the adduct is rapidly quenched to stable products without decomposing back to the reactants. In this case, the measured rate coefficient appears to be independent on pressure. Figure 5 shows the reaction rate of propene as a function of total number density which is proportional to pressure. The black points are the data from Vakhtin et.al. ${ }^{72}$ and red points are the data taken during the validation of the experimental set up in the new laboratory. At low pressure, the reaction rate increases with the pressure whereas at high pressure, the reaction rate is independent of pressure.

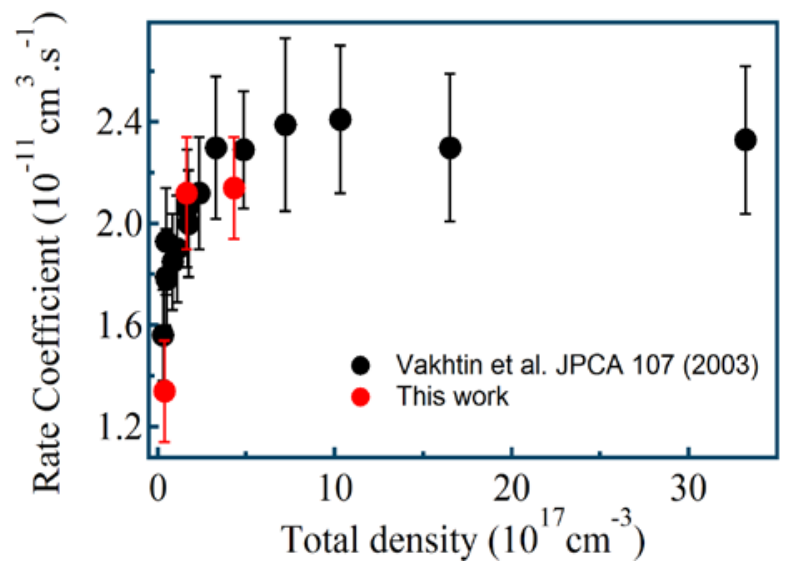

Figure 5. The reaction rate of propene as a function of total density of the gas

\subsection{First Ring Formation and Carbon Molecular Growth}

The formation of the first aromatic ring, benzene, from aliphatic radicals and molecules in combustion environments is a crucial step toward the formation of polycyclic aromatic hydrocarbons. The most widely accepted route for the formation of benzene is the self- 
recombination of propargyl $\left(\mathrm{C}_{3} \mathrm{H}_{3}\right)$ radicals. ${ }^{42,76-81}$ This step is believed to be the rate limiting step during the formation of polycyclic aromatic hydrocarbons. Scheme 12 shows the formation of benzene or phenyl radical from two propargyl radicals as suggested by the above studies.

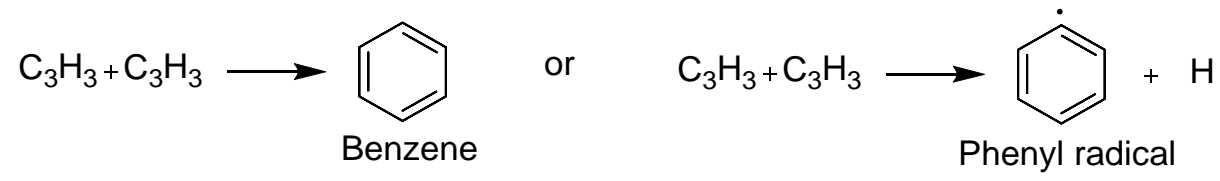

Scheme 12. Propargyl radical self recombines to form benzene or phenyl radical

The ease of benzene formation from the recombination of propargyl radicals is attributed to the barrierless pathway. Based on theoretical calculations by Georgievskii et.al. ${ }^{47}$, the exit channel of the recombination reaction of propargyl radicals is barrierless. In addition, the potential energy surface calculated by Miller and Klippenstein ${ }^{48,82}$ shows low-energy reaction paths with no energy barrier from the recombination reaction of propargyl leading to benzene or phenyl $+\mathrm{H}$.

The first ring benzene may also be formed by the hydrogen-atom assisted isomerization of fulvene. In scheme 13 (i) a propargyl radical combines with an allyl radical to form fulvene $\left(\mathrm{C}_{5} \mathrm{H}_{4} \mathrm{CH}_{2}\right)$. Under fuel-rich conditions, $\mathrm{H}$ atom assisted isomerization leads to benzene formation. ${ }^{83,84}$ (ii) Cyclopentadiene reacts with $\mathrm{CH}_{3}$ to form $\mathrm{C}_{5} \mathrm{H}_{4} \mathrm{CH}_{3}$ which loses an $\mathrm{H}$ atom to form fulvene. This leads to the formation of benzene by hydrogen atom assisted isomerization. ${ }^{85-}$ 87 
(i)

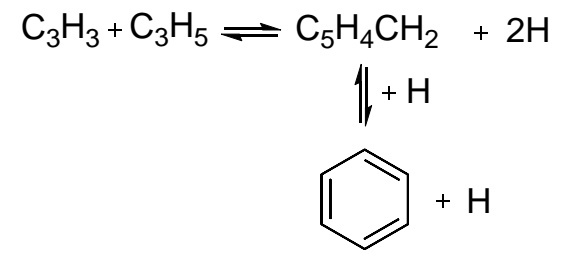

(ii)

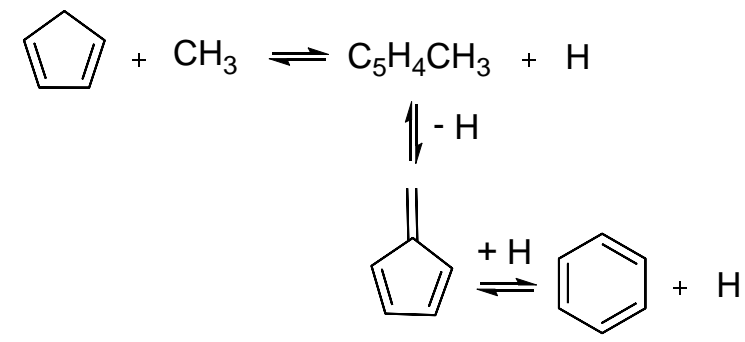

Scheme 13. Hydrogen-atom assisted isomerization of fulvene to benzene

Alternatively, the propargyl radical may react with abundant gaseous acetylene (building block) to form cyclopentadienyl $\left(\mathrm{c}-\mathrm{C}_{5} \mathrm{H}_{5}\right)$ radical. This cyclopentadienyl radical reacts rapidly and forms benzene as shown in the scheme 14 below. ${ }^{85}$

$$
\mathrm{C}_{3} \mathrm{H}_{3}+\mathrm{C}_{2} \mathrm{H}_{2} \rightarrow \mathrm{c}-\mathrm{C}_{5} \mathrm{H}_{5}+\mathrm{CH}_{3} \rightarrow \mathrm{C}_{6} \mathrm{H}_{7}+\mathrm{H} \rightarrow \mathrm{C}_{6} \mathrm{H}_{6} \text { (benzene) }+2 \mathrm{H}
$$

Scheme 14. The reaction of propargyl radical with acetylene to generate benzene

\subsection{Formation of Polycyclic Aromatic Hydrocarbons (PAHs)}

Polycyclic aromatic hydrocarbons (PAHs) are the organic compounds made up of carbons and hydrogens forming two or more fused aromatic ring structures. They can be categorized into two types: low molecular weight and high molecular weight PAHs. ${ }^{28}$ Those $^{2}$ containing less than four fused or condensed rings e.g., naphthalene, anthracene, phenanthrene, etc. are low molecular weight PAHs and those containing more than four fused aromatic rings e.g., pyrene, benzo (a)-anthracene, etc. are high molecular weight PAHs. According to Masih et.al. ${ }^{88}$ their physical properties vary depending on their size and structure. In general, pure PAHs 
are crystalline and lipophilic in nature implying they dissolve well in organic solvents. They are stable, light sensitive, heat and corrosion resistant. ${ }^{88}$ Being most stable at high temperature in the combustion environment, they are called stabilomers. ${ }^{89}$ They have a specific UV absorption spectrum. The fluorescence excitation and emission wavelengths in nanometers of some PAHs are given in the table 3 .

Table 3. Some PAHs and their fluorescence excitation and emission wavelengths

\begin{tabular}{lll}
\hline PAH & Excitation wavelength (nm) & Emission wavelength (nm) \\
\hline Naphthalene $^{90}$ & 273 & 333 \\
Phenanthrene $^{90}$ & 297 & 340 \\
Anthracene $^{90}$ & 295 & 430 \\
Pyrene $^{90}$ & 260 & 430 \\
Benzo (a)-anthracene $^{90}$ & 260 & 430 \\
Chrysene $^{90}$ & 260 & 430 \\
\hline
\end{tabular}

The major sources of PAHs in our atmosphere are transportation vehicles that use various fuels. ${ }^{90,91}$ Figure 6 shows the structures of two, three and four-ringed PAHs formed during fuel burning processes.<smiles>c1ccc2ccccc2c1</smiles>
Naphthalene $\left(\mathrm{C}_{10} \mathrm{H}_{8}\right)$<smiles>c1cc2ccc3cccc4ccc(c1)c2c34</smiles><smiles>c1ccc2cc3c(ccc4ccccc43)cc2c1</smiles><smiles>c1ccc2c(c1)ccc1ccccc12</smiles>

Phenanthrene $\left(\mathrm{C}_{14} \mathrm{H}_{10}\right)$<smiles>c1ccc2c(c1)ccc1c3ccccc3ccc21</smiles>

Chrysene $\left(\mathrm{C}_{18} \mathrm{H}_{12}\right)$

Figure 6. Structures of two, three, and four ringed PAHs 


\subsubsection{The HACA Mechanism}

Hydrocarbons growth in combustion environment occurs through the accepted "HACA" mechanism. HACA stands for "H-abstraction- $\mathrm{C}_{2} \mathrm{H}_{2}$-addition” (hydrogen-abstraction-acetyleneaddition). ${ }^{92}$ This mechanism proceeds through repetitive two steps: (i) abstraction of a hydrogen atom and (ii) addition of an acetylene molecule. The abstraction often occurs though the reaction with a hydrogen atom to form $\mathrm{H}_{2}$ and a hydrocarbon radical. ${ }^{41,51}$ In scheme 15 gaseous hydrogen atom abstracts hydrogen atom from the benzene ring to form the phenyl radical hydrogen.

$$
\mathrm{H} \rightleftharpoons+\mathrm{H}_{2}
$$

Scheme 15. Benzene reacts with gaseous hydrogen atom to form phenyl radical

An acetylene molecule can then add to the phenyl radical to form phenylacetylene through $\mathrm{H}$-atom elimination. Phenylacetylene can further react to give the naphthalene and acenaphthylene as shown in scheme $16 .^{93}$

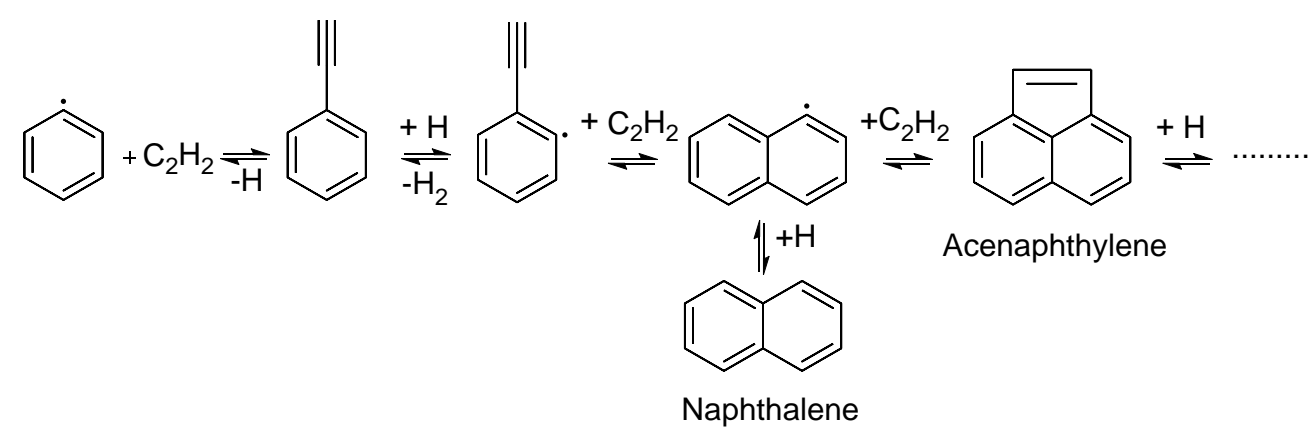

Scheme 16. The phenyl radical leading to the formation of naphthalene and acenaphthylene 
This process can repeat until the formation of two or peri-condensed (three or more) rings. The entire process is thermodynamically driven by the formation of hydrogen molecule and the loss of hydrogen. ${ }^{92}$

This HACA mechanism has been recently observed experimentally by Parker et.al ${ }^{94}$ who probed phenylacetylene intermediate and naphthalene using photoionization mass spectrometry under combustion-like environment at 300 Torr pressure and $1020 \pm 100 \mathrm{~K}$ temperature. Scheme 17 shows the HACA reaction pathway proposed by Parker et al. for the formation of naphthalene After the formation of the phenyl radical, its reaction with acetylene forms the styrenyl radical. Further reaction with acetylene forms naphthalene. In this mechanism there is no need for second $\mathrm{H}$-abstraction from phenylacetylene. Alternatively, o-vinylphenyl radical can be formed by isomerization of styrenyl radical. O-vinylphenyl radical on reacting with acetylene forms naphthalene.

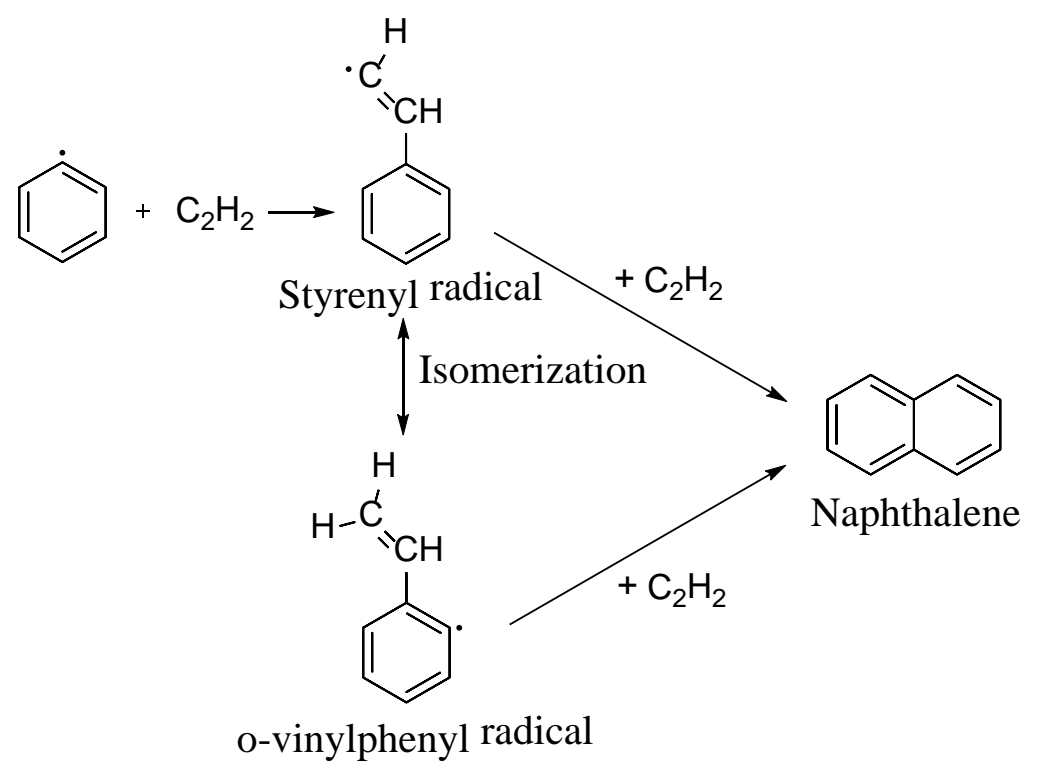

Scheme 17. Formation of naphthalene through the HACA mechanism 
Benzene can also react with the phenyl radical to form biphenyl as shown in scheme 18 . Further reaction with acetylene can lead to the formation of phenanthrene. ${ }^{92,95,96}$
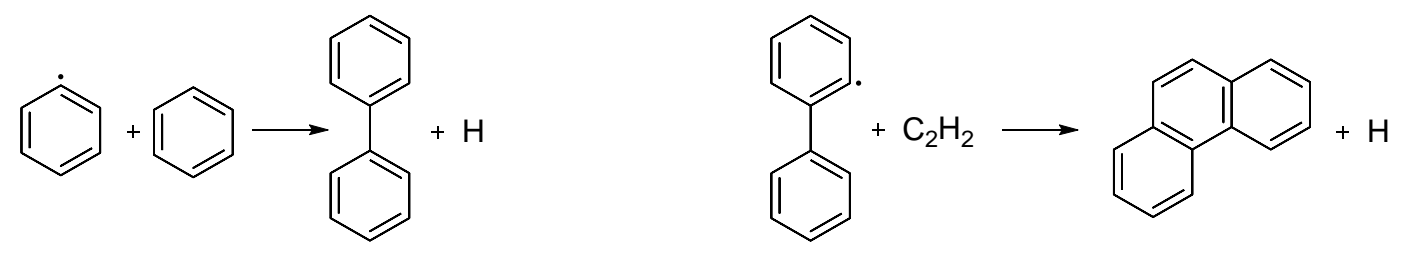

Scheme 18. Phenyl radical reacting with benzene to form biphenyl and biphenyl radical reacting with acetylene to form phenanthrene

The HACA mechanism can also be initiated through reaction with other radicals. Scheme 19 depicts the formation of large polycyclic molecules initiated by the addition of the $\mathrm{OH}$ radical on phenylacetylene. ${ }^{97}$ The initially formed adduct may react with gaseous acetylene to form a naphthyl radical through water elimination. This naphthyl radical can again react with gaseous acetylene to form acenaphthylene or lead to the pathway forming phenanthrenyl radical.

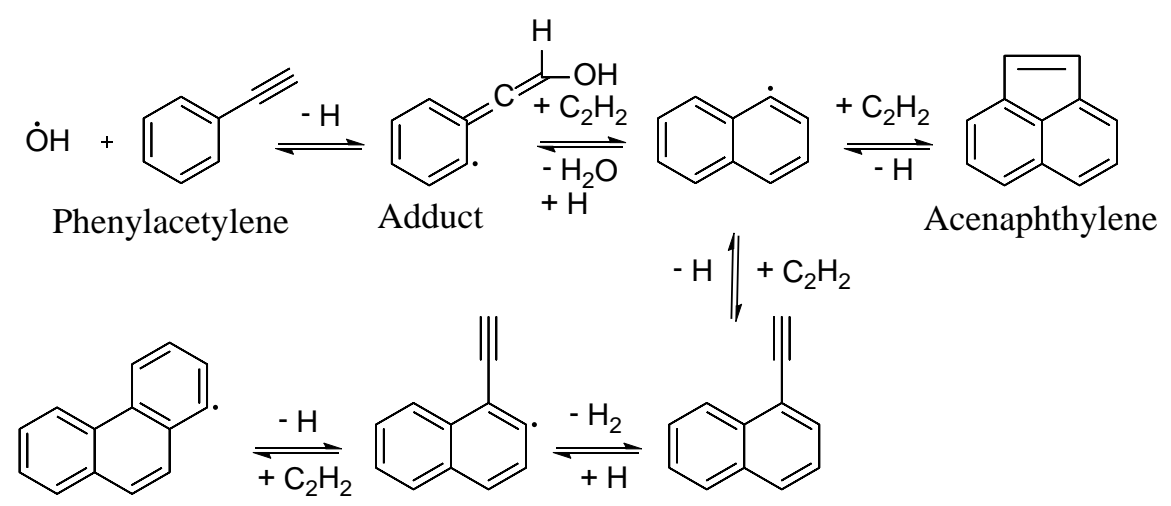

Scheme 19. Hydroxyl radical reacting with phenylacetylene to form phenanthrenyl radical 


\subsubsection{Formation of Soot}

Figure 7 shows a proposed schematic representation of the gas phase and heterogeneous schemes leading to the formation of soot in combustion environments. ${ }^{52}$ The formation of soot is initiated in the molecular zone. This zone is the chemically rich area where small radicals (e.g. $\mathrm{C}_{3} \mathrm{H}_{3}, \mathrm{CH}, \mathrm{OH}$ ) and molecules (e.g., aliphatic hydrocarbons) are formed. These small radicals and molecules react with abundant fuel molecules to form aromatic molecules such as benzene in case of aliphatic fuels such as methane, ethylene, acetylene or butane. ${ }^{52,98}$ In case of aromatic fuels, it breaks down into large concentrations of acetylene and benzene. Benzene might react with C2 and C3 species such as acetylene to form large fused ring aromatic hydrocarbons called polycyclic aromatic hydrocarbons (PAH described in section 1.10).

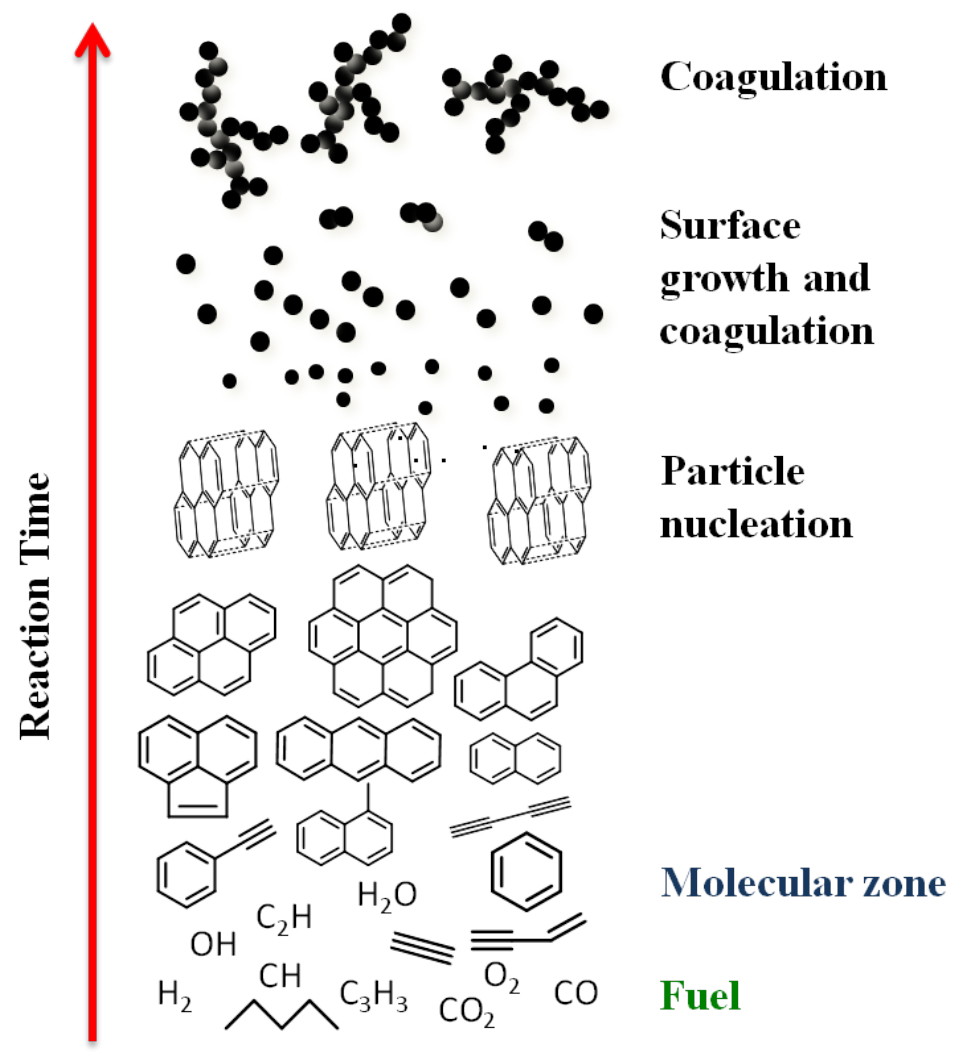

Figure 7. Proposed scheme of soot formation (adapted from Richter and Howard ${ }^{52}$ ) 
The smaller PAHs grow into heavy PAHs by reacting with other reactive species. These heavy aromatic molecules might condense near the particle nucleation region to form nascent soot particles. This means in the particle nucleation region, PAH species begin to collide and stick to each other forming PAH dimers. ${ }^{92}$ PAH dimers collide with PAH molecules to form PAH trimers or with other PAH dimers to form PAH tetramers. This process continues and the individual PAH size keeps increasing via molecular growth reactions. Eventually these PAH clusters evolve into solid (soot) particles. ${ }^{92}$ Soot particles are believed to have surface radical sites reacting with gas phase molecules such as acetylene or large aromatic radicals resulting in surface growth. Soot particles coagulate via particle-particle collisions resulting into aggregates.

The size of soot particles emitted from vehicles varies from 10 to 150 nanometers. Rose et.al..$^{99}$ measured the size of soot particles from 30 to $150 \mathrm{~nm}$ but the major fraction of soot were found around $80 \mathrm{~nm}$. Karjalainen et.al. ${ }^{100}$ investigated the size distribution of exhaust particles in modern gasoline vehicles and found two modes of non-volatile exhaust particles, one with mean size of $30 \mathrm{~nm}$ and the other with mean size of $70 \mathrm{~nm}$. Their results indicated that these particle modes consisted of soot but with different morphology. According to Kim et.al. ${ }^{101}$ the size of soot particles was less than $100 \mathrm{~nm}$ for both diesel and gasoline automobiles.

\subsection{Resonance-Stabilized Radicals (RSRs): Alternative Pathways for PAH Formation}

\subsubsection{Definition and Characteristics}

Based on the experimental and kinetic modeling of PAH formation in methane and ethylene flames, Melius et.al. ${ }^{85}$ concluded that the HACA process cannot account for the amount of PAHs formed in flames. Resonance stabilized radicals have recently been proposed as an alternative route to PAH formation.

Resonance stabilized radicals have an unpaired electron distributed over multiple sites in 
the molecule resulting in several electronic structures called resonance structures. Due to the delocalization of the unpaired electron over the molecule, resonance stabilized radicals form weaker bonds with stable molecules (e.g., oxygen) ${ }^{78,102}$ compared to other ordinary radicals. These weak addition complexes are neither stabilized by collisional quenching nor support rearrangement. $^{82}$ So RSRs are relatively unreactive and accumulate at high temperature in combustion environments.

The difference between the energy of the actual radical and its possible resonance structures is the resonance energy. The extra stability makes the radical thrive in harsh conditions such as combustion environment where they can accumulate in large concentrations. ${ }^{98}$ Because the resonance energy of the radicals may be lost upon their reaction with other radicals or molecules they are resistant to oxidation and thermal decomposition. ${ }^{53}$ The most common resonance stabilized radicals in combustion are propargyl $\left(\mathrm{C}_{3} \mathrm{H}_{3}\right)$, allyl $\left(\mathrm{C}_{3} \mathrm{H}_{5}\right)$, cyclopentadienyl $\left(\mathrm{C}_{5} \mathrm{H}_{5}\right)$, fulvenallenyl $\left(\mathrm{C}_{7} \mathrm{H}_{5}\right)$, and benzyl $\left(\mathrm{C}_{6} \mathrm{H}_{5} \mathrm{CH}_{2}\right)$ radicals. Scheme 20 shows the resonance structures and respective hybrid structure of fulvenallenyl radical. The real structure of the radical is an average of all the resonance structures. The stability of fulvenallenyl radical is due to the conjugated propargyl and cyclopentadiene units, ${ }^{103}$ making it the most stable $\mathrm{C}_{7} \mathrm{H}_{5}$ isomer.

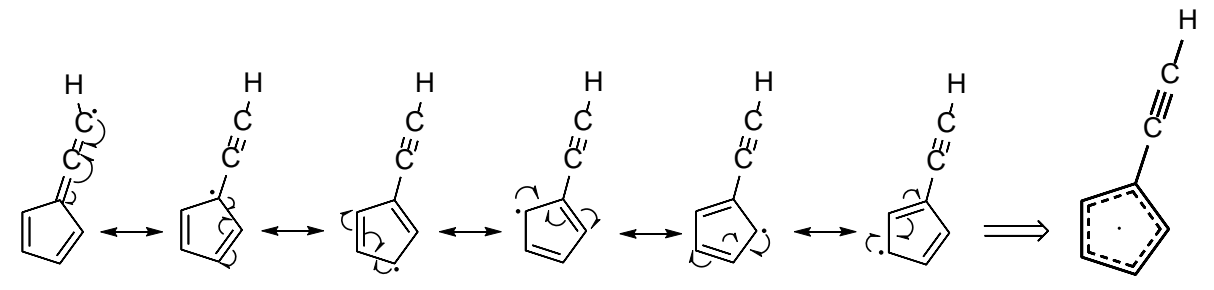

Scheme 20. Resonance structures of fulvenallenyl radical

Some resonance-stabilized radicals such as cyclopentadienyl and fulvenallenyl have recently been considered as precursors to polycyclic aromatic hydrocarbon formation through 
self and cross recombination. The increase in credibility on these radicals is credited to their high thermal stability and resistance to oxidation in flames while remaining reactive enough.

\subsubsection{Current Knowledge on Resonance Stabilized Radical Reactivity}

At high concentrations and assuming the rapid reaction rates, RSR may self- or crosscombine forming larger molecules. Scheme 21 shows the self-recombination of propargyl $\left(\mathrm{C}_{3} \mathrm{H}_{3}\right)$ radicals to form $\mathrm{C}_{6} \mathrm{H}_{6}$ isomers at room temperature. ${ }^{104}$ Depending on the orientation of the radicals, it can yield different products.

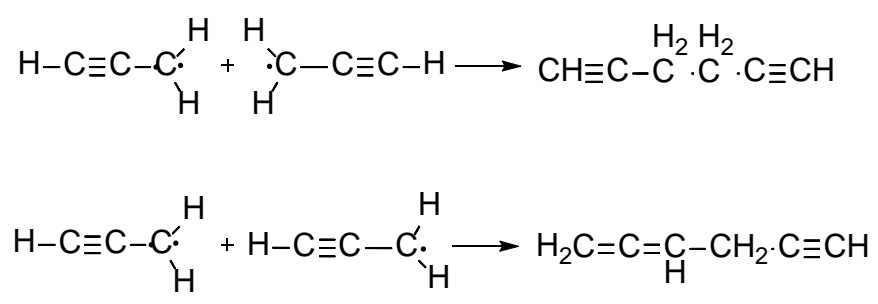

Scheme 21. Two propargyl radicals forming 1,5-hexadiyne and 1,2-hexadiene-5-yne

Miller and Klippenstein ${ }^{82}$ predicted theoretically that at higher temperature $(>1200 \mathrm{~K})^{82}$ the combination orientation could be either head-head or tail-tail and could form benzene, fulvene, and phenyl radical by eliminating $\mathrm{H}$ atom as shown in scheme 22 .

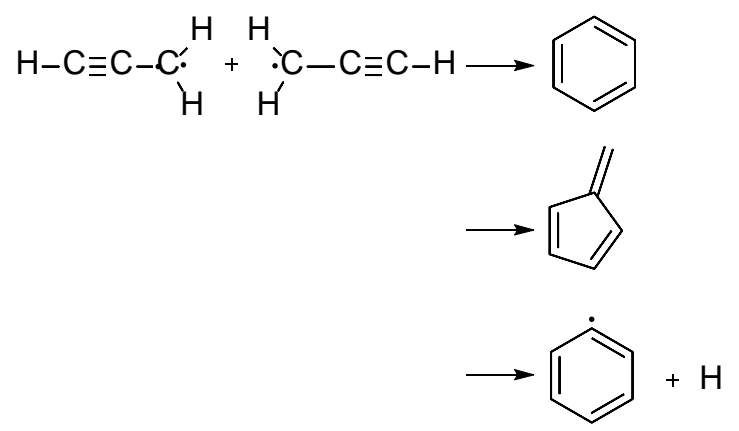

Scheme 22. Two propargyl radicals forming benzene, fulvene, and phenyl radical 
Miller et.al. ${ }^{84}$ predicted the formation of benzene through cross-combination of the propargyl radical with the allyl radical followed by elimination of hydrogen atoms as shown in scheme $23 .{ }^{84}$

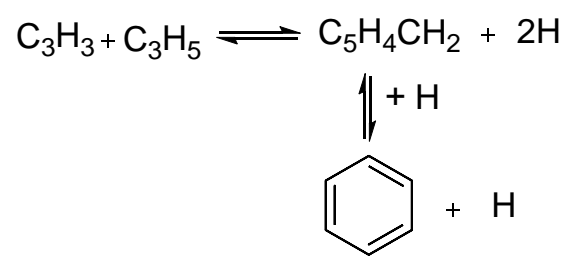

Scheme 23. Propargyl radical combines with allyl radical to form benzene via fulvene

Other cross-recombination products have also been predicted as shown in scheme 24 by Georgievskii et.al. ${ }^{47}$

$$
\begin{aligned}
& \mathrm{C}_{3} \mathrm{H}_{3}+\mathrm{C}_{3} \mathrm{H}_{5} \rightarrow \mathrm{CH}_{2} \mathrm{CCHCH}_{2} \mathrm{CHCH}_{2} \\
& \mathrm{C}_{3} \mathrm{H}_{3}+\mathrm{C}_{3} \mathrm{H}_{5} \rightarrow \mathrm{CHCCH}_{2} \mathrm{CH}_{2} \mathrm{CHCH}_{2}
\end{aligned}
$$

Scheme 24. Cross combination of RSRs to form addition products

RSR can also combine with other carbon containing radicals. The experimental work of Fahr and Nayak ${ }^{104}$ showed the combination of $\mathrm{C}_{3} \mathrm{H}_{3}$ with $\mathrm{CH}_{3}$ yielded 1-butyne $\left(\mathrm{CH} \equiv \mathrm{C}-\mathrm{CH}_{2}-\right.$ $\left.\mathrm{CH}_{3}\right)$ and 1,2-butadiene $\left(\mathrm{CH}_{2}=\mathrm{C}=\mathrm{CH}-\mathrm{CH}_{3}\right){ }^{104}$

\subsubsection{Previous Experiments and Computational Studies on Resonance Stabilized Radicals}

Cyclopentadienyl is one of the most important RSRs with a resonance stabilization energy of $22 \mathrm{Kcal} / \mathrm{mole}^{105}$. Melius et. al. ${ }^{85}$ investigated the recombination reaction mechanism of two cyclopentadieyl radicals theoretically. In their study, naphthalene is formed from two 
cyclopentadienyl radicals via the formation of $\mathrm{C}_{10} \mathrm{H}_{10}$ and $\mathrm{C}_{10} \mathrm{H}_{9}$ intermediates as shown in scheme 25 .

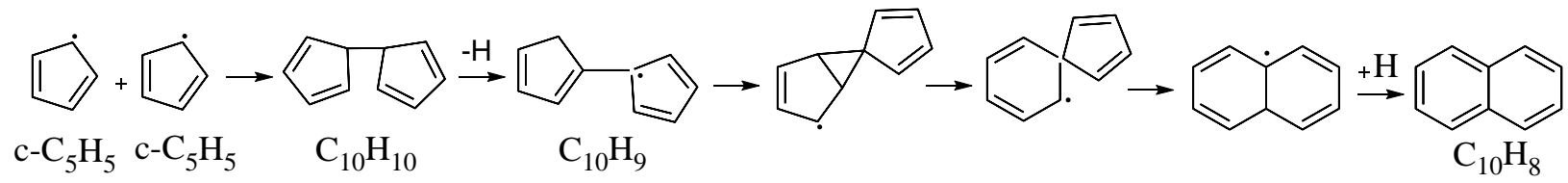

Scheme 25. Two cyclopentadienyl radicals form naphthalene

Mebel and Kilsov ${ }^{54}$ investigated the recombination reaction of two cyclopentadienyl radicals using various theoretical and computational methods. The formation of naphthalene occurs through the formation of 9,10-dihydrofulvalene $\left(\mathrm{C}_{10} \mathrm{H}_{10}\right)$ and 9-H-fulvalenyl $\left(\mathrm{C}_{10} \mathrm{H}_{9}\right)$ which is favorable at temperatures below $1000 \mathrm{~K}$. Scheme 26 shows the mechanism proposed by Mebel and Kilsov. ${ }^{54}$ for the combination of two cyclopentadienyl radicals to form naphthalene $\left(\mathrm{C}_{10} \mathrm{H}_{8}\right)+\mathrm{H}$ and fulvalene $\left(\mathrm{C}_{10} \mathrm{H}_{8}\right)+\mathrm{H}$. This channel is recommended for kinetic modeling.

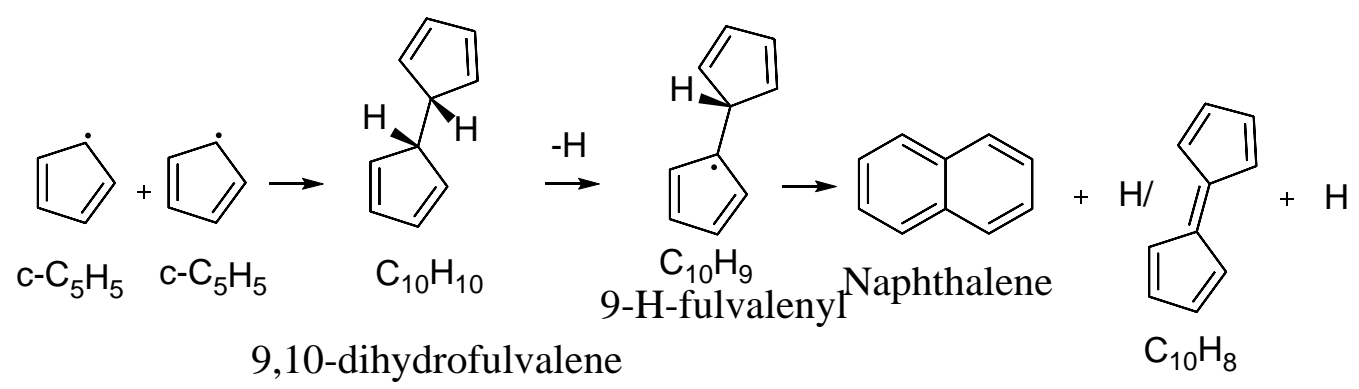

Scheme 26 Two cyclpentadienyl radicals form naphthalene and fulvalene

Cavalotti and Polino ${ }^{55}$ investigated the new pathway for the recombination of cyclopentadienyl radicals theoretically using density functional theory and CBS-QB3. Scheme 27 shows their proposed $\mathrm{C}_{10} \mathrm{H}_{10}$ adduct formation from two cyclpentadienyl radicals that follows 
the route of azulyl radicals to form naphthalene. This channel is predicted to be dominant below 1200 K. The pathway from azulene to naphthalene has been well paved by Alder et.al. ${ }^{106}$ and Stirling et.al. ${ }^{107}$ For the azulyl radical, the radical is shown in one site however it can be in different site.

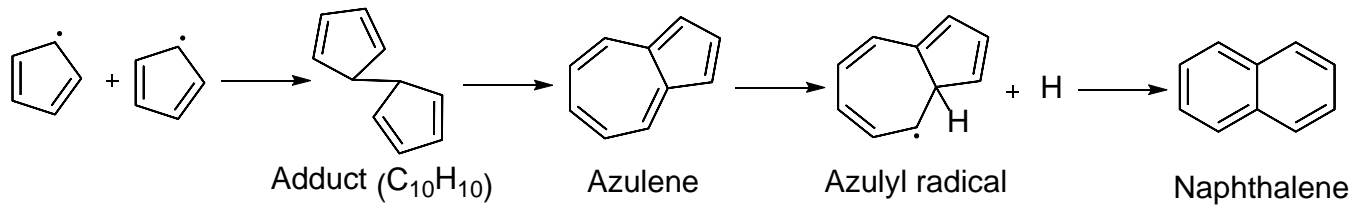

Scheme 27. Two cyclopentadienyl radicals react to form naphthalene

To my knowledge there is only one experimental work to date on the self-reaction of cyclopentadienyl radical. Knyazev and Popov ${ }^{108}$ studied the reaction kinetics of selfrecombination of cyclopentadienyl radicals using laser photolysis/photoionization mass spectroscopy over the 304-600 K temperature range. The radicals were generated by laser photolysis of cyclopentadiene at $248 \mathrm{~nm}$. The room temperature reaction rate was found to be $(3.98 \pm 0.41) \times 10^{-10} \mathrm{~cm}^{3} \mathrm{~s}^{-1}$ and the reaction showed negative temperature dependence over their experimental range. Below $600 \mathrm{~K} \mathrm{C}_{10} \mathrm{H}_{10}$ intermediate dominates whereas above $800 \mathrm{~K}$ gas chromatography/mass spectrometry show two isomers of $\mathrm{C}_{10} \mathrm{H}_{8}$ viz. naphthalene being the major product and azulene the minor one as shown in the scheme 28. 
(i)

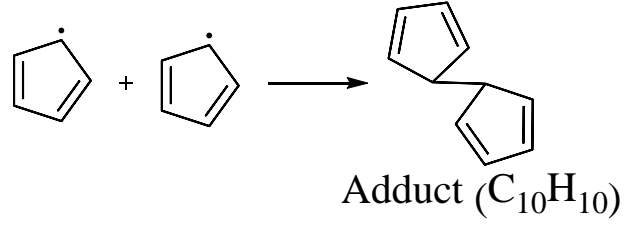

(ii)
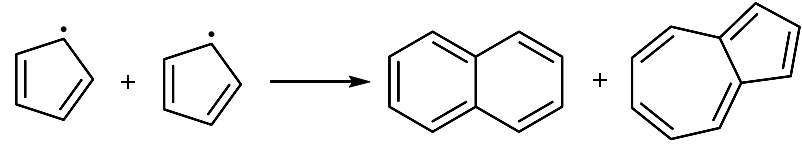

Naphthalene Azulene

Scheme 28. (i) Adduct $\left(\mathrm{C}_{10} \mathrm{H}_{10}\right)$ and (ii) naphthalene and azulene in the study by Knyazev and popov ${ }^{108}$

Another important RSR in combustion is fulvenallenyl which is formed from either dissociation or weak $\mathrm{H}$ atom abstraction from fulvenallene. ${ }^{109}$ da Silva et.al. ${ }^{57}$ studied the decomposition of fulvenallenyl radical theoretically and experimentally. The major dissociation products were found to be propargyl and diacetylene $\left(\mathrm{C}_{4} \mathrm{H}_{2}\right)$.

Da Silva and Bozzeli ${ }^{53}$ proposed self-reaction and cross-reaction of fulvenallenyl radical with propargyl and cyclopentadienyl radicals to form PAHs such as phenanthrene, naphthalene and diphenyl. Scheme 29 (i) and (ii) are cross-combination reactions of fulvenallenyl with propargyl and cyclpentadienyl yielding naphthalene and diphenyl respectively. Scheme 29 (iii) is the self-recombination of fulvenallenyl radicals yielding phenanthrene. 
(i)

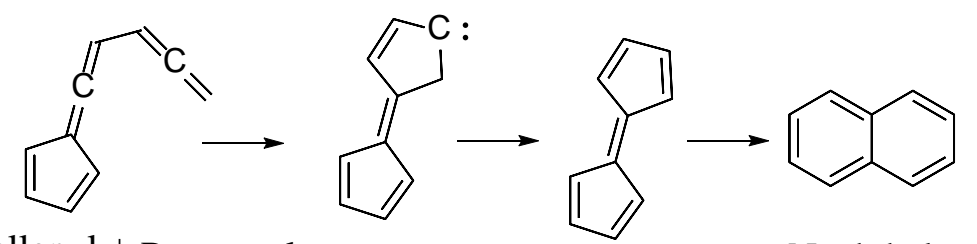

Fulvenallenyl + Propargyl

Naphthalene

(ii)

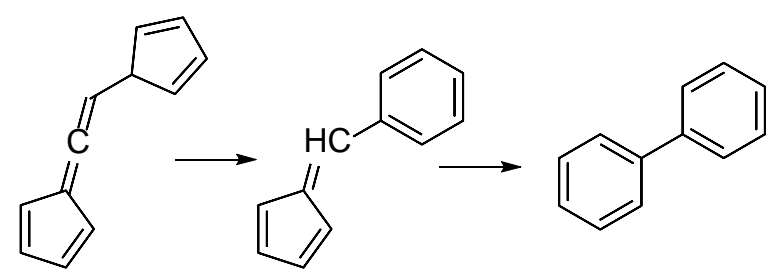

Fulvenallenyl + Cyclopentadienyl

Diphenyl

(iii)

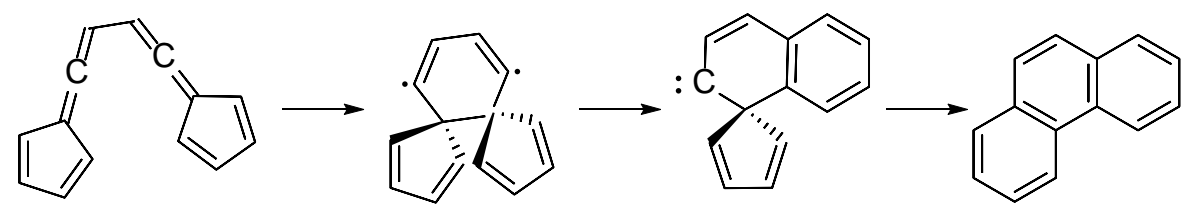

Fulvenallenyl + Fulvenallenyl

Phenanthrene

Scheme 29. (i) Fulvenalenyl and propargyl form naphthalene (ii) fulvenallenyl and cyclopentadienyl form diphenyl and (iii) self-recombination of fulvenallenyl form phenanthrene

\subsubsection{What Else do We Need to Know?}

Soot formation can only be understood by identifying key reactions leading to their molecular precursors and investigating their reaction rates experimentally. Along with the formation of benzene, the recombination of resonance-stabilized radicals is believed to be the alternative routes for $\mathrm{PAH}$ formation. ${ }^{53,108}$ However, there is still a limited number of experimental studies on their reaction kinetics due to their high reactivity and the difficulty of generating them in known concentrations. ${ }^{108}$ Despite these difficulties, there are experimental studies on self-reactions on propargyl radicals ${ }^{80,104}$ and cyclopentadienyl radicals. ${ }^{108}$ In addition Knyavev and Popov ${ }^{108}$ have demonstrated that RSR recombination reaction can have strong negative temperature dependent. Furthermore the radical-radical reactions are pressure 
dependent. For example Howe and Fahr ${ }^{110}$ observed significant pressure and temperature dependencies on propargyl self-reaction.

The significance of the fulvenallenyl radical reactions is suggested from theoretical studies but there is not even a single experimental study on this radical. So generating fulvenallenyl radical and studying its self and cross radical reaction kinetics at relevant pressure and temperature is crucial at this point. It would give more insight into the understanding of the role of fulvenallenyl resonance stabilized radical in the formation of polycyclic aromatic hydrocarbon (PAH).

\subsection{Relevance of My Research}

In one of the studies, ${ }^{111}$ the reaction of the hydroxyl radical was investigated with phenylacetylene over the 1-7.5 Torr (133.32-999.92 Pa) pressure and 298-423 K temperature ranges using pulsed laser photolysis-laser induced fluorescence (PLP-LIF) technique. Phenylacetylene is a known PAH formation precursor. The reaction was found to be pressure and temperature independent suggesting the formation of an addition intermediate. Based on the exothermicity calculation of the intermediates using Gaussian 09, CBS-QB3, the addition of OH to the outer carbon of $-\mathrm{C}_{2} \mathrm{H}$ in phenylacetylene is the most likely reaction outcome. Furthermore, this product is stabilized by resonance, with four resonance structures as shown in scheme 30 .

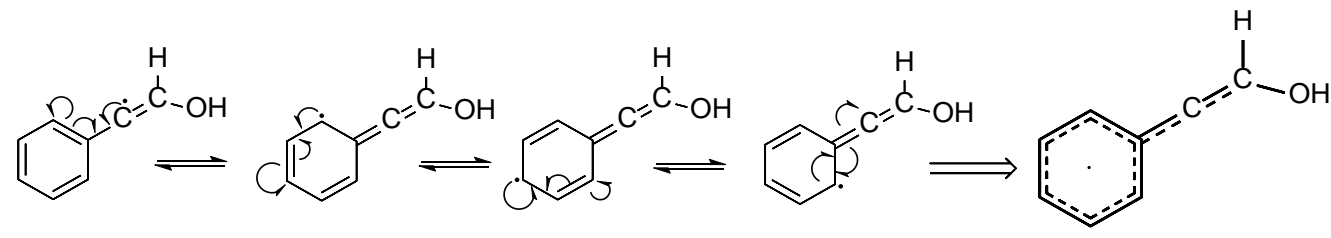

Scheme 30. Resonance structures of $\mathrm{OH}$-phenylacetylene adduct 
This motivated us to study the reaction of hydroxyl radical with fulvenallene-another compound believed to contribute to PAH formation. da Silva et.al..$^{53}$ suggested that $\mathrm{OH}$ could abstract the loosely bound hydrogen atom from fulvenallene to form fulvenallenyl, a resonance stabilized radical in combustion. This reaction was studied over 298-450 K at 5 Torr using PLPLIF technique. The reaction rate coefficient was independent of pressure but showed negative temperature dependence. This suggests an association mechanism dominant at room and lower temperature whereas abstraction is likely to dominate at temperature higher than $450 \mathrm{~K}$. This indicates that the fulvenallenyl radical is likely to be formed at higher temperature in combustion environment. To confirm the formation of fulvenallenyl radical, higher temperature kinetic studies of $\mathrm{OH}$ with fulvenallene are needed in order to determine the contribution of fulvenallenyl radical in PAH formation. Self and cross-combination reactions of fulevenallenyl radicals (resonance-stabilized radicals) also need to be investigated over a wide range of temperatures.

\section{References:}

(1) Mishra, D. P. Experimental combustion : an introduction, 2014.

(2) Where the Energy Goes: Gasoline Vehicles, https://www.fueleconomy.gov/feg/atv.shtml; Department of Energy.

(3) Keating, E. L. Applied combustion; CRC Press/Taylor \& Francis: Boca Raton, 2007; Vol. 206.;206;.

(4) Srivastava, S. P.; Hancsók, J.; ebrary, I. Fuels and fuel-additives; Wiley: Hoboken, New Jersey, 2013.

(5) DOE: Fossil Fuels: How Fossil Fuels Were Formed 2016.

(6) Liberman, M. A. Introduction to Physics and Chemistry of Combustion: Explosion, Flame, Detonation; Springer: Berlin, 2008; Vol. 1. Aufl.;1;.

(7) Mohr, S. H.; Wang, J.; Ellem, G.; Ward, J.; Giurco, D. Fuel 2015, 141, 120.

(8) Miller, B. G. M. S.; Books24x, I. Clean coal engineering technology; ButterworthHeinemann: Burlington, MA, 2011.

(9) Schobert, H. H.; ebrary, I. Chemistry of fossil fuels and biofuels; Cambridge University Press: New York;Cambridge [England];, 2013.

(10) Mokhatab, S.; Poe, W. A.; Speight, J. G.; Gulf Professional Pub.: Burlington, MA, 2006.

(11) Pogaku, R.; Sarbatly, R. H.; Ebooks, C. Advances in biofuels; Springer: New York, 2013; Vol. 2. 
(12) Naik, S. N.; Goud, V. V.; Rout, P. K.; Dalai, A. K. Renewable \& Sustainable Energy Reviews 2010, 14, 578.

(13) Chaudhary, G.; Singh, L. K.; Ghosh, S. Bioresource Technology 2012, 124, 111.

(14) Chen, Y.; Cheng, J. J.; Creamer, K. S. Bioresource Technology 2008, 99, 4044.

(15) Balat, M. Energy Exploration \& Exploitation 2007, 25, 195.

(16) Balat, M.; Balat, H. Applied Energy 2009, 86, 2273.

(17) Ferguson, C. R.; Kirkpatrick, A. 2015.

(18) Stone, R. Introduction to internal combustion engines; SAE International: Warrendale, PA., 2012.

(19) Roth A.C., F. B. J., and Scott W.; Goodheart Willcox: 2012.

(20) Andreae, M. O.; Merlet, P. Global Biogeochemical Cycles 2001, 15, 955.

(21) Ferguson, C. R.; Kirkpatrick, A. T. Internal Combustion Engines : Applied Thermosciences (3rd Edition); Wiley: Somerset, NJ, USA, 2015.

(22) Kirchstetter, T. W.; Harley, R. A.; Kreisberg, N. M.; Stolzenburg, M. R.; Hering, S. V. Atmospheric Environment 1999, 33, 2955.

(23) Wang, F.; Li, P.; Zhang, J.; Mei, Z.; Mi, J.; Wang, J. International Journal of Hydrogen Energy 2015, 40, 6228.

(24) Liberman, M. A.; Springer: Berlin, 2008.

(25) Smith, K. R.; Frumkin, H.; Balakrishnan, K.; Butler, C. D.; Chafe, Z. A.; Fairlie, I.; Kinney, P.; Kjellstrom, T.; Mauzerall, D. L.; McKone, T. E.; McMichael, A. J.;

Schneider, M. In Annual Review of Public Health, Vol 34; Fielding, J. E., Ed. 2013; Vol. 34, p 159.

(26) Naeher, L. P.; Brauer, M.; Lipsett, M.; Zelikoff, J. T.; Simpson, C. D.; Koenig, J. Q.; Smith, K. R. Inhalation Toxicology 2007, 19, 67.

(27) Unwin, J.; Cocker, J.; Scobbie, E.; Chambers, H. Annals of Occupational Hygiene 2006, 50, 395.

(28) Kim, K.-H.; Jahan, S. A.; Kabir, E.; Brown, R. J. C. Environment International 2013, 60, 71.

(29) Yao, M.; Zheng, Z.; Liu, H. Progress in Energy and Combustion Science 2009, 35, 398.

(30) Musculus, M. P. B.; Miles, P. C.; Pickett, L. M. Progress in Energy and Combustion Science 2013, 39, 246.

(31) Charalambides, A. G. In Advances in Internal Combustion Engines and Fuel Technologies; Ng, H. K., Ed.; InTech: 2013.

(32) Jun, D.; Ishii, K.; Iida, N. Jsme International Journal Series B-Fluids and Thermal Engineering 2003, 46, 60.

(33) Love, N.; Parthasarathy, R. N.; Gollahalli, S. R. International Journal of Green Energy 2011, 8, 113.

(34) Tinaut, F. V.; Reyes, M.; Gimenez, B.; Pastor, J. V. Energy \& Fuels 2011, 25, 119.

(35) Yu, X.; Yang, Z.; Peng, J.-B.; Zhang, L.; Ma, Y.-F.; Yang, C.-B.; Li, X.-H.; Sun, R. Chinese Physics B 2015, 24.

(36) Luque, J.; Jeffries, J. B.; Smith, G. P.; Crosley, D. R. Combustion and Flame 2001, 126, 1725.

(37) Zhou, B.; Brackmann, C.; Li, Z.; Alden, M. Combustion and Flame 2015, 162, 368.

(38) Ledesma, E. B.; Marsh, N. D.; Sandrowitz, A. K.; Wornat, M. J. Proceedings of the Combustion Institute 2002, 29, 2299. 
(39) Taatjes, C. A.; Hansen, N.; Osborn, D. L.; Kohse-Hoeinghaus, K.; Cool, T. A.; Westmoreland, P. R. Physical Chemistry Chemical Physics 2008, 10, 20.

(40) Wang, K.; Villano, S. M.; Dean, A. M. Combustion and Flame 2015, 162, 4456.

(41) Wang, H.; Frenklach, M. Combustion and Flame 1997, 110, 173.

(42) Marinov, N. M.; Pitz, W. J.; Westbrook, C. K.; Vincitore, A. M.; Castaldi, M. J.; Senkan, S. M.; Melius, C. F. Combustion and Flame 1998, 114, 192.

(43) Norinaga, K.; Janardhanan, V. M.; Deutschmann, O. International Journal of Chemical Kinetics 2008, 40, 199.

(44) Pitz, W. J.; Mueller, C. J. Progress in Energy and Combustion Science 2011, 37, 330.

(45) Battin-Leclerc, F. Progress in Energy and Combustion Science 2008, 34, 440.

(46) Hansen, N.; Miller, J. A.; Kasper, T.; Kohse-Hoeinghaus, K.; Westmoreland, P. R.;

Wang, J.; Cool, T. A. Proceedings of the Combustion Institute 2009, 32, 623.

(47) Georgievskii, Y.; Miller, J. A.; Klippenstein, S. J. Physical Chemistry Chemical Physics 2007, 9, 4259.

(48) Miller, J. A.; Klippenstein, S. J. Journal of Physical Chemistry A 2003, 107, 7783.

(49) Goulay, F.; Leone, S. R. Journal of Physical Chemistry A 2006, 110, 1875.

(50) Woon, D. E. Chemical Physics 2006, 331, 67.

(51) Frenklach, M.; Warnatz, J. Combustion Science and Technology 1987, 51, 265.

(52) Richter, H.; Howard, J. B. Progress in Energy and Combustion Science 2000, 26, 565.

(53) da Silva, G.; Bozzelli, J. W. Journal of Physical Chemistry A 2009, 113, 12045.

(54) Mebel, A. M.; Kislov, V. V. Journal of Physical Chemistry A 2009, 113, 9825.

(55) Cavallotti, C.; Polino, D. Proceedings of the Combustion Institute 2013, 34, 557.

(56) Polino, D.; Famulari, A.; Cavallotti, C. Journal of Physical Chemistry A 2011, 115, 7928.

(57) da Silva, G.; Trevitt, A. J.; Steinbauer, M.; Hemberger, P. Chemical Physics Letters 2011, 517, 144.

(58) Zhang, T.; Zhang, L.; Hong, X.; Zhang, K.; Qi, F.; Law, C. K.; Ye, T.; Zhao, P.; Chen, Y. Combustion and Flame 2009, 156, 2071.

(59) Hansen, N.; Kasper, T.; Klippenstein, S. J.; Westmoreland, P. R.; Law, M. E.; Taatjes, C. A.; Kohse-Hoeinghaus, K.; Wang, J.; Cool, T. A. Journal of Physical Chemistry A 2007, $111,4081$.

(60) Li, Y.; Zhang, L.; Tian, Z.; Yuan, T.; Wang, J.; Yang, B.; Qi, F. Energy \& Fuels 2009, 23, 1473.

(61) Cavallotti, C.; Derudi, M.; Rota, R. Proceedings of the Combustion Institute 2009, 32, 115.

(62) da Silva, G.; Cole, J. A.; Bozzelli, J. W. Journal of Physical Chemistry A 2009, 113, 6111.

(63) Derudi, M.; Polino, D.; Cavallotti, C. Physical Chemistry Chemical Physics 2011, 13, 21308.

(64) Buckingham, G. T.; Ormond, T. K.; Porterfield, J. P.; Hemberger, P.; Kostko, O.; Ahmed, M.; Robichaud, D. J.; Nimlos, M. R.; Daily, J. W.; Ellison, G. B. Journal of Chemical Physics 2015, 142.

(65) Shapero, M.; Cole-Filipiak, N. C.; Haibach-Morris, C.; Neumark, D. M. Journal of Physical Chemistry A 2015, 119, 12349.

(66) Gligorovski, S.; Strekowski, R.; Barbati, S.; Vione, D. Chemical Reviews 2015, 115, 13051.

(67) Atkinson, R.; Arey, J. Chemical Reviews 2003, 103, 4605. 
(68) Atkinson, R. Chemical Reviews 1986, 86, 69.

(69) Gierczak, T.; Talukdar, R. K.; Herndon, S. C.; Vaghjiani, G. L.; Ravishankara, A. R. Journal of Physical Chemistry A 1997, 101, 3125.

(70) Srinivasan, N. K.; Su, M. C.; Sutherland, J. W.; Michael, J. V. Journal of Physical Chemistry A 2005, 109, 1857.

(71) Tyndall, G. R. S.; Orlando, J. J.; Wallington, T. J.; Hurley, M. D.; Goto, M.; Kawasaki, M. Physical Chemistry Chemical Physics 2002, 4, 2189.

(72) Vakhtin, A. B.; Murphy, J. E.; Leone, S. R. Journal of Physical Chemistry A 2003, 107, 10055.

(73) Morris, E. D.; Stedman, D. H.; Niki, H. Journal of the American Chemical Society 1971, 93, 3570.

(74) Wollenhaupt, M.; Crowley, J. N. Journal of Physical Chemistry A 2000, 104, 6429.

(75) Tully, F. P.; Ravishankara, A. R.; Thompson, R. L.; Nicovich, J. M.; Shah, R. C.; Kreutter, N. M.; Wine, P. H. Journal of Physical Chemistry 1981, 85, 2262.

(76) Hansen, N.; Miller, J. A.; Klippenstein, S. J.; Westmoreland, P. R.; Kohse-Hoeinghaus, K. Combustion Explosion and Shock Waves 2012, 48, 508.

(77) Kern, R. D.; Singh, H. J.; Wu, C. H. International Journal of Chemical Kinetics 1988, 20, 731.

(78) Miller, J. A.; Melius, C. F. Combustion and Flame 1992, 91, 21.

(79) Pope, C. J.; Miller, J. A. Proceedings of the Combustion Institute 2000, 28, 1519.

(80) Shafir, E. V.; Slagle, I. R.; Knyazev, V. A. Journal of Physical Chemistry A 2003, 107, 8893.

(81) Wu, C. H.; Kern, R. D. Journal of Physical Chemistry 1987, 91, 6291.

(82) Miller, J. A.; Klippenstein, S. J. Journal of Physical Chemistry A 2001, 105, 7254.

(83) Hansen, N.; Li, W.; Law, M. E.; Kasper, T.; Westmoreland, P. R.; Yang, B.; Cool, T. A.; Lucassen, A. Physical Chemistry Chemical Physics 2010, 12, 12112.

(84) Miller, J. A.; Klippenstein, S. J.; Georgievskii, Y.; Harding, L. B.; Allen, W. D.; Simmonett, A. C. Journal of Physical Chemistry A 2010, 114, 4881.

(85) Melius, C. F.; Colvin, M. E.; Marinov, N. M.; Pitz, W. J.; Senkan, S. M. Reaction mechanisms in aromatic hydrocarbon formation involving the C5H5 cyclopentadienyl moiety, 1996.

(86) Ikeda, E.; Tranter, R. S.; Kiefer, J. H.; Kern, R. D.; Singh, H. J.; Zhang, Q. Proceedings of the Combustion Institute 2000, 28, 1725.

(87) Moskaleva, L. V.; Mebel, A. M.; Lin, M. C. The CH3+C5H5 reaction: A potential source of benzene at high temperatures, 1996.

(88) Masih, J.; Masih, A.; Kulshrestha, A.; Singhvi, R.; Taneja, A. Journal of Hazardous Materials 2010, 177, 190.

(89) Stein, S. E.; Fahr, A. Journal of Physical Chemistry 1985, 89, 3714.

(90) Harrison, R. M.; Smith, D. J. T.; Luhana, L. Environmental Science \& Technology 1996, 30, 825.

(91) Marr, L. C.; Kirchstetter, T. W.; Harley, R. A.; Miguel, A. H.; Hering, S. V.; Hammond, S. K. Environmental Science \& Technology 1999, 33, 3091.

(92) Frenklach, M. Physical Chemistry Chemical Physics 2002, 4, 2028.

(93) Richter, H.; Benish, T. G.; Mazyar, O. A.; Green, W. H.; Howard, J. B. Proceedings of the Combustion Institute 2000, 28, 2609. 
(94) Parker, D. S. N.; Kaiser, R. I.; Troy, T. P.; Ahmed, M. Angewandte Chemie-International Edition 2014, 53, 7740.

(95) Frenklach, M.; Clary, D. W.; Gardiner Jr, W. C.; Stein, S. E. Symposium (International) on Combustion 1988, 21, 1067.

(96) Frenklach, M.; Yuan, T.; Ramachandra, M. K. Energy \& Fuels 1988, 2, 462.

(97) D'Anna, A.; Violi, A.; D'Alessio, A. Combustion and Flame 2000, 121, 418.

(98) Miller, J. A.; Pilling, M. J.; Troe, E. Proceedings of the Combustion Institute 2005, 30, 43.

(99) Rose, D.; Wehner, B.; Ketzel, M.; Engler, C.; Voigtlander, J.; Tuch, T.; Wiedensohler, A. Atmospheric Chemistry and Physics 2006, 6, 1021.

(100) Karjalainen, P.; Pirjola, L.; Heikkila, J.; Lahnde, T.; Tzamkiozis, T.; Ntziachristos, L.; Keskinen, J.; Ronkko, T. Atmospheric Environment 2014, 97, 262.

(101) Kim, W. S.; Kim, S. H.; Lee, D. W.; Lee, S.; Lim, C. S.; Ryu, J. H. Environmental Science \& Technology 2001, 35, 1005.

(102) Atkinson, D. B.; Hudgens, J. W. Journal of Physical Chemistry A 1999, 103, 4242.

(103) Chakraborty, A.; Fulara, J.; Maier, J. P. Angewandte Chemie-International Edition 2016, 55, 228.

(104) Fahr, A.; Nayak, A. International Journal of Chemical Kinetics 2000, 32, 118.

(105) Zhong, X.; Bozzelli, J. W. International Journal of Chemical Kinetics 1997, $29,893$.

(106) Alder, R. W.; East, S. P.; Harvey, J. N.; Oakley, M. T. Journal of the American Chemical Society 2003, 125, 5375.

(107) Stirling, A.; Iannuzzi, M.; Laio, A.; Parrinello, M. Chemphyschem 2004, 5, 1558.

(108) Knyazev, V. D.; Popov, K. V. Journal of Physical Chemistry A 2015, 119, 7418.

(109) Polino, D.; Cavallotti, C. Journal of Physical Chemistry A 2011, 115, 10281.

(110) Howe, P. T.; Fahr, A. Journal of Physical Chemistry A 2003, 107, 9603.

(111) Kailasanathan, R. K. A.; Thapa, J.; Goulay, F. Journal of Physical Chemistry A 2014, $118,7732$. 


\section{Chapter 2: Experimental Methods}

\subsection{Vacuum and Gas Set Up}

The reactions of hydroxyl radical with polycyclic aromatic hydrocarbon precursors were studied at pressures ranging from of 1-10 Torr (rough or low vacuum region, 1-760 Torr or 133.32-1.01 $\times 10^{5} \mathrm{~Pa}$ ) in a buffer gas of helium. Ultra-high purity (UHP) grade (99.99\%) helium gasses from Matheson Tri Gas were regulated using Harris CGA E-4 9296 multi-stage regulator and Matheson regulator (model no. 3539-350) and connected to MKS mass flow controllers using 1/4" copper tube. The major experimental components used for these experiments are discussed in the following sub-sections.

\subsubsection{The Reaction Chamber}

The experiments were conducted in a stainless steel 6-way-cross chamber. A schematic of the reaction chamber is shown in Figure 1. All six ports of the reaction chamber had 1.5" inner diameter and are 2 " in length. Four of these ports were horizontal and two were vertical. Two of the four horizontal ports were extended by connecting optical ports of 1 " inner diameter and 4" in length through CF flanges. One of the horizontal ports was extended by connecting a constricted stainless steel port of $0.4^{\prime \prime}$ inner diameter and 8 " in length. The remaining horizontal port was first connected to a stainless steel tee, $1.5^{\prime \prime}$ in diameter and 3 " in length for connection to the vacuum pump. All the horizontal ports had $1 / 4^{\prime \prime}$ tubes for the injection of gas injection and pressure measurements using capacitance manometer (Baratron MKS instruments). The lower vertical port was closed using a stainless steel blank flange. The upper vertical port had a quartz window for UV-light transmission.

The four horizontal ports described above for laser access were terminated by a 1" uncoated UV fused silica windows held by Viton O-rings and Teflon rings. The windows were at 
Brewster angle $\left(53^{\circ}\right)$ in order to reduce reflection of the vertically polarized laser beam. A plano concave mirror was placed (fixed) in the lower inner portion of the lower vertical port to collect the signal from the radicals.

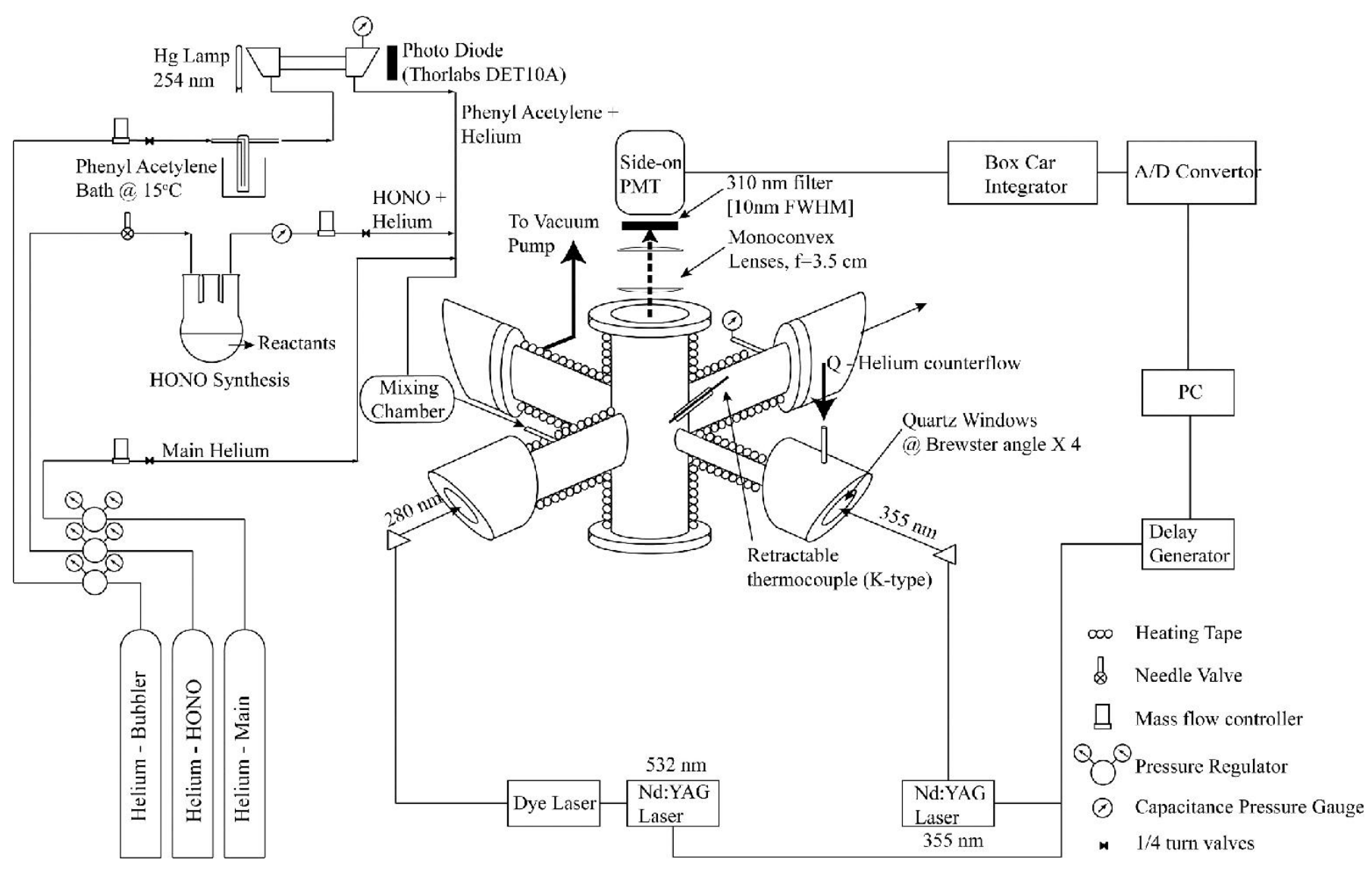

Figure 1. Experimental schematic for the kinetic measurements from 300 to $450 \mathrm{~K}$ and 1 to 10 Torr

\subsubsection{Vacuum Pump and Gas Injection}

The required dynamic vacuum of 1-10 Torr at a constant total mass flow rate of helium buffer gas was achieved by using a high volume flow rate vacuum pump. The vacuum pump and gas injection method are discussed in the following sub-sections. 


\subsubsection{Vacuum Pump}

Vacuum was maintained in the reaction chamber using an Edwards iQDP80/iQMB500 mechanical booster combination also called Roots Blower. The iQMB is a mechanical booster connected to the inlet of a iQDP rotary backing pump. Both pumps are water cooled through a circulation of chilled water. ${ }^{1}$ The Roots Blower is a dry pump that avoids contamination of the system with a low maintenance cost. ${ }^{2}$ It is designed for working in harsh environments requiring high pumping speeds, while minimizing contamination from pump oil. Its operating pressure range is between roughing (1-760 Torr) and high-vacuum (down to $10^{-5}$ Torr) ${ }^{2,3}$ independently of gas composition. Its compression ratio (ratio of outlet to inlet pressure) ranges from $10-50 .^{3}$ The pumping capacity of the Roots blower used in these experiments is $600 \mathrm{~m}^{3} \mathrm{hr}^{-1}$ (cubic meter per hour).

Roots Blower is a valveless, rotary positive displacement pump. This means the Roots Blower operates by forcing the fixed volume of gas from its inlet pressure section to its discharge zone. It has two 8-shaped rotors interlocked and synchronized rotating in opposite directions with a rotation speed of the order of $3000 \mathrm{rpm}$ (rotations per minute). The space between two rotors is approximately $0.2 \mathrm{~mm}$. The rotors do not contain any oil, making it suitable for applications requiring high gas purity. Figure 2 shows the two lobed rotors and the arrowheads show their movement during the operation. The rotation of the 8-shaped rotors in opposite direction traps the air within the spaces surrounding the lobes forcing it into the outlet section. ${ }^{4}$ 


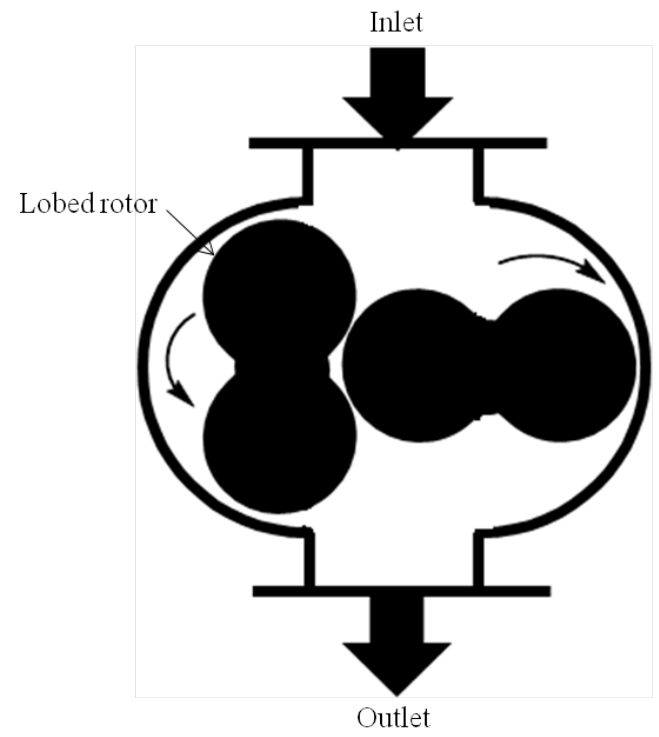

Figure 2. Lobed rotors in Roots Blower vacuum pump ${ }^{4}$

The pressure in the reaction chamber was regulated using a butterfly valve placed between the reaction chamber and the flexible stainless-steel tube that was connected to the vacuum chamber.

\subsubsection{Injection of Gases}

All the gaseous reactants (propylene, butane, benzene vapors in He, acetaldehyde vapors in He, fulvenallene vapors in $\mathrm{He}$ ) and gaseous radical precursors (HONO) were mixed in the stainless steel mixing cylinder of volume $50 \mathrm{~cm}^{3}$ placed before the reaction chamber and introduced into the reaction chamber. Helium was used as the main carrier gas to avoid any reaction with the free radicals. The flow rates of all gases were monitored and controlled by calibrated mass flow controllers (MKS Instruments).

Liquid reactants such as phenylacetylene, were placed inside of a bubbler and maintained at constant temperature using a regulated oil bath (1016S, ISOTEMP) at $15^{\circ} \mathrm{C}$. For benzene, oil 
bath was used at room temperature. A fraction of the carrier gas (from 5 to 10\%) was bubbled through the liquid to obtain a saturated gas flow. In the same way, vapors of the hydroxyl radical precursor-liquid hydrogen peroxide (30 and $50 \% \mathrm{w} / \mathrm{w}$ )-was placed inside of a bubbler which temperature was regulated using a water bath. The fraction of the carrier gas flowing through the bubbler ranged from 5 to $10 \%$ of the total flow.

\subsubsection{Calibration of Mass Flow Controllers (MFCs)}

Depending on their flow rate capacity mass flow controllers (MFCs) were calibrated using custom-made cylinders of known volume (i. radius=2.5" and height=12.5", ii. radius=2.5" and height=25.5" or iii. radius=2.5" and height=47.75") initially evacuated to pressure below 0.1 Torr. A set flow rate of helium was flown through the MFC into one of the cylinders. The pressure was measured using capacitance manometer and recorded as a function of time. The standard flow rate $q_{v}^{0}$ through the controller was determined from the slope $\dot{P}$ of the pressure vs. time using equation 1 :

$q_{v}^{0}=\dot{P} \cdot \frac{V}{P^{0}} \cdot \frac{T^{0}}{T}$

$V$ the volume of the cylinder and tubings, $P^{0}$ is the standard pressure $\left(1.013 \times 10^{5} \mathrm{~Pa}\right), T^{0}$ is the standard temperature $(273.15 \mathrm{~K})$ and $T$ the temperature used for calibration (room temperature $=300 \mathrm{~K})$.

The above process was repeated for several set flow rates of helium over the entire range of the MFC. The calibration factors of the MFC were determined from the slope of the measured flow rates versus the set flow rates. Figure 3 shows the measured flow rate plotted against the set flow rates for a MFC (full range 200 sccm, serial number 021869896). The experimental points 
were fitted with a line resulting in a slope of 0.81215 and an offset of -0.41667 (sccm). The actual flow rate of the mass flow controller was obtained using $Q_{a c t u a l}^{0}=0.81215 \times \mathrm{Q}_{\text {read }^{-}}^{0}$ 0.41667(sccm).

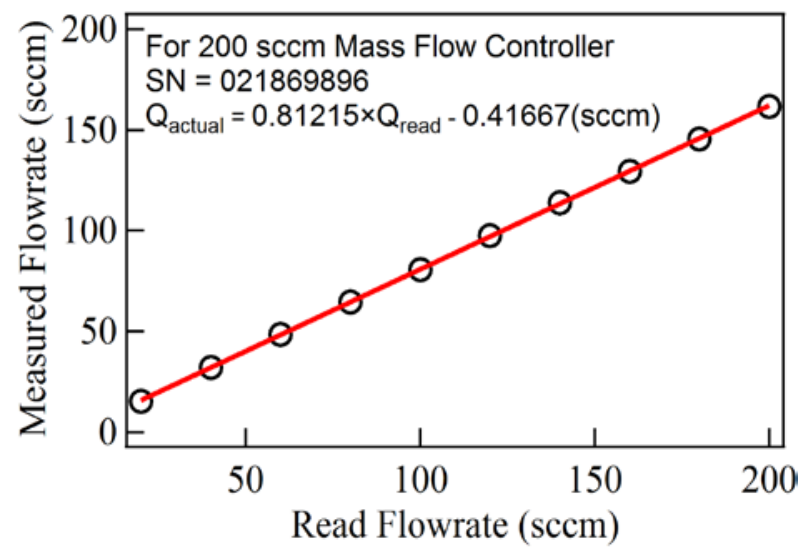

Figure 3. Plot of measured flowrate vs read flowrate for the $200 \mathrm{sccm}$ mass flow controller

\subsubsection{Determination of Buffer Gas Total Flow Rate and Average Linear Velocity}

Because of the pulsed nature of the pump-probe technique used for the studies, it was necessary to assure that the entire reaction chamber $\left(V=500 \mathrm{~cm}^{3}\right)$ was refreshed with buffer gas every laser pulse, every $100 \mathrm{~ms}$. The minimum volume flow rate $Q_{v}$ required to refresh the cell every 100 ms was

$\mathrm{Q}_{\mathrm{V}}=\frac{500 \mathrm{~cm}^{3}}{100 \times 10^{-3} \mathrm{~s}}=\frac{500 \times 10^{-6} \mathrm{~m}^{3}}{100 \times 10^{-3} \mathrm{~s}}=5 \times 10^{-3} \mathrm{~m}^{3} \mathrm{~s}^{-1}=18 \mathrm{~m}^{3} \mathrm{hr}^{-1}$

The standard flow rate can be written as

Standard flow rate $Q_{v}^{0}$ of an ideal gas at standard pressure $\left(P^{0}\right)$ and temperature $\left(T^{0}\right)$ is given by:

$Q_{V}^{0}=\frac{d V^{0}}{d t}=\dot{V}^{0}=\frac{d}{d t}\left(\frac{n R T^{0}}{P^{0}}\right)=\frac{\dot{n} R T^{0}}{P^{0}}$ 
where $n$ is the number of moles of gas and $\mathrm{R}$ the gas constant. The molar flow rate $\dot{n}$ and the mass flow rate $\dot{m}$ are related by:

$$
\dot{\mathrm{n}}=\frac{\dot{\mathrm{m}}}{\mathrm{M}}
$$

where $\mathrm{M}$ is the molar mass of the gas. Plugging equation 3 in 2 we obtain

$$
\mathrm{Q}_{\mathrm{v}}^{0}=\frac{\mathrm{Q}_{\mathrm{m}}}{\rho}=\dot{\mathrm{m}} \frac{\mathrm{RT}^{0}}{\mathrm{MP}^{0}}=\mathrm{Q}_{\mathrm{m}} \frac{\mathrm{RT}^{0}}{\mathrm{MP}^{0}}
$$

with $\rho^{0}$ density of the gas at standard pressure and temperature and $Q_{m}$ the mass flow rate.

Similarly, under the experimental conditions of pressure $P$ and temperature $T$ :

$$
\mathrm{Q}_{\mathrm{m}}=\frac{\mathrm{PM}}{\mathrm{RT}} \mathrm{Q}_{\mathrm{v}}
$$

Incorporating $\mathrm{Q}_{\mathrm{m}}$ from equation 5 in 4 results in

$$
\mathrm{Q}_{\mathrm{v}}^{0}=\frac{\mathrm{P}}{\mathrm{P}^{0}} \cdot \frac{\mathrm{T}^{0}}{\mathrm{~T}} \cdot \mathrm{Q}_{\mathrm{v}}
$$

At $\mathrm{P}=5$ Torr, and $\mathrm{T}=300 \mathrm{~K}$ (typical experimental conditions) we obtained $\mathrm{Q}_{\mathrm{v}}^{0}=1797.0 \mathrm{sccm}$ (standard cubic centimeter per minute). The experimental value was $1.8 \mathrm{slm}$ (standard litter per minute). Values at lower pressure were obtained by normalizing by the cell pressure, $Q_{v}^{\mathbf{0}}=3.6$ $\operatorname{slm}$ at 10 Torr.

An estimate of linear velocity of the buffer gas in the reaction cell can be obtained using $Q_{v}=v S$ with $S$ the cross-section in the middle region of the reaction chamber (2 cm diameter). This leads to the velocity of the buffer gas of about $3.98 \mathrm{~ms}^{-1}$. The size of the optical detection region in the cell is determined by the diameter of the photolysis laser pulses, approximately 0.5 $\mathrm{cm}$. Therefore the resident time $\mathrm{t}$ of the reactants in the detection region is: 
$\mathrm{t}=\frac{0.5 \times 10^{-2}}{3.98}=1.26 \times 10^{-3} \mathrm{~s}=1.26 \mathrm{~ms}$

In order to approximate the cell to a static cell, the reaction time is kept shorter than the resident time.

\subsubsection{High Temperature Set Up}

For the high temperature set up, the reaction chamber was wrapped with a constantwattage heat cable and insulated using fiberglass. Temperature dependence of the rate coefficient was investigated over the 298-450 K temperature range. The gas temperature in the center of the reaction chamber was measured using a retractable Type $\mathrm{K}$ thermocouple. Temperatures at the inlet of the reaction chamber and at the point of pressure measurement were also monitored using two Type $\mathrm{K}$ thermocouples. The temperature control circuit used controllers (OmegaCN710), and solid-state relays (SSR330AC) to regulate power to the heat cables. Before every experiment the reaction chamber was allowed to thermalize for about an hour, minimizing fluctuations in measured temperatures.

\subsection{The Pulsed Laser Photolysis-Laser Induced Fluorescence (PLP-LIF) Technique}

\subsubsection{Background}

The pulsed laser photolysis-laser induced fluorescence (PLP-LIF) technique has been extensively used to study the reaction kinetics of short lived species ${ }^{5,6}$ (radicals/molecules) that are important in combustion ${ }^{6-11}$, atmospheric ${ }^{12-15}$, and interstellar medium ${ }^{7,8,16,17}$ chemistry. In this technique, a short laser pulse ( 10 ns or less) initiates the reaction by breaking the bond of the radical precursor to generate radicals (e.g. $\mathrm{CHBr}_{3}+3 \mathrm{hv} \rightarrow \mathrm{CH}+3 \mathrm{Br}$ ). ${ }^{18}$ The most commonly used photolysis lasers are excimer (193 nm and $248 \mathrm{~nm}$ ) and Nd:YAG (266 nm and 
$355 \mathrm{~nm}$ ). The other tunable laser (dye laser) monitors the radical concentration using Laser Induced Fluorescence (LIF). The beams of the photolysis and probe lasers usually propagate perpendicular to each other, as shown in Figure 1. This leads to a detection region of about $\sim 3$ $\mathrm{mm}$.

This pump-probe PLP-LIF technique became popular since early 1980s. Owing to the broad implication and spectrum in the research area of reaction kinetics, this technique has been widely used over the last decades. Robertshaw et.al. ${ }^{19}$ investigated the reaction of hydroxyl radical with nitrogen dioxide. $\mathrm{OH}$ radicals were generated using pulsed laser photolysis of nitric acid $\left(\mathrm{HNO}_{3}\right)$ and by monitoring the decay of $\mathrm{OH}$ radicals by laser induced fluorescence at excitation wavelength of $282 \mathrm{~nm}$ and observing its emission wavelength at $310 \mathrm{~nm}$. Perry ${ }^{9}$ studied the reaction of NCO radical, an important intermediate in fuel and flame with hydrogen and nitric oxide. ${ }^{9}$ In this study, an excimer laser was employed to photolyze HNCO at $248 \mathrm{~nm}$ to generate NCO radical and a tunable dye laser was used for NCO LIF at excitation wavelength of $416.8 \mathrm{~nm}$ and monitored its fluorescence at $438.5 \mathrm{~nm} .{ }^{9}$ Blitz et.al. ${ }^{20}$ studied the reaction of $\mathrm{CH}$ radical that is important in combustion and planetary atmospheres with $\mathrm{H}_{2} \mathrm{O}$ using PLP-LIF. CH radicals were generated by photolyzing $\mathrm{CHBr}_{3}$ at $248 \mathrm{~nm}$ using an excimer laser and monitored the concentration of $\mathrm{CH}$ by LIF using tunable dye laser at excitation wavelength $430 \mathrm{~nm}$ (A $\mathrm{A}^{2} \Delta \leftarrow \mathrm{X}^{2} \Pi$ ) observing its fluorescence at $490 \mathrm{~nm} .{ }^{20}$ Similarly, Rudich et.al. ${ }^{21}$ studied the reaction of hydroxyl radical with methyl butenol, an important natural volatile organic compound (NVOC) in the atmosphere. In this study, OH radicals were generated from HONO using pulsed laser photolysis (frequency tripled Nd:YAG laser) at 355nm and the concentration of $\mathrm{OH}$ radicals were monitored using tunable dye laser exciting at $281.1 \mathrm{~nm}$ and monitoring its fluorescence at $308.8 \mathrm{~nm}$. 


\subsubsection{Pulsed Photolysis}

In pulsed photolysis short-lived radicals are produced in high concentrations instantaneously using an intense pulse with high peak power in the UV or visible region. ${ }^{22}$ The laser has a very short pulse (in the order of nanoseconds), shorter than the lifetime of the radicals or intermediates. The laser is a well collimated beam. ${ }^{22}$ The monochromatic light of the laser ensures selective photolysis of the radical precursor. ${ }^{23}$ Fixed wavelength lasers such as Nd:YAG (532 nm, $355 \mathrm{~nm}$, and $266 \mathrm{~nm}$ ) and excimer (193 nm and $248 \mathrm{~nm}$ ) are the most widely used pulsed sources for photolysis. Here the fourth harmonic $(266 \mathrm{~nm})$ of a Nd:YAG laser was used to break the weak O-O bond $(196 \mathrm{KJ} / \mathrm{mol})^{24}$ in $\mathrm{H}_{2} \mathrm{O}_{2}$ to generate two $\mathrm{OH}$ radicals as shown in $\mathrm{R} 1$ below. The third harmonic (355 nm) of the same Nd:YAG laser was used to photolyze HONO ( $200 \mathrm{KJ} / \mathrm{mol})^{25,26}$ as shown in $\mathrm{R} 2$ below and generate $\mathrm{OH}$ radicals with limited photolysis of the hydrocarbon precursor. ${ }^{27,28}$ Alternatively excimer laser (193 nm and $248 \mathrm{~nm}$ ) can be used to generate $\mathrm{OH}$ radicals from $\mathrm{H}_{2} \mathrm{O}_{2}{ }^{29}$

$$
\begin{aligned}
& \mathrm{H}_{2} \mathrm{O}_{2}+\mathrm{hv}(266 \mathrm{~nm}) \rightarrow 2 \mathrm{OH} \\
& \mathrm{HONO}+\mathrm{hv}(355 \mathrm{~nm}) \rightarrow \mathrm{OH}+\mathrm{NO}
\end{aligned}
$$

\subsubsection{Laser Induced Fluorescence (LIF)}

Laser induced fluorescence (LIF) is the emission of photons from the species (radicals or molecules) following the absorption of a laser pulse. In LIF, light radiation from a tunable laser is tuned to a wavelength matching the absorption line of the species. Upon absorption of the resonant excitation wavelength, the species goes from a lower electronic state to a higher excited electronic state with a short lifetime $\left(10^{-5}\right.$ to $\left.10^{-10} \mathrm{~s}\right)$. The excited state then relaxes spontaneously 
to a lower vibronic state. In the case that the returning state is different than the initial state, the emitted radiation has a different wavelength than that of the excitation radiation. The broadband off-resonance fluorescence is observed using a bandpass filter. ${ }^{30}$ As the species are excited at their resonant wavelength, the absorption cross-section is larger providing higher sensitivity of the species with very low concentration (ppm level).

Figure 4 shows a schematic of the laser induced fluorescence scheme of hydroxyl radical $(\mathrm{OH})$. Hydroxyl radical initially in their ground $\mathrm{X}^{2} \Pi$ state and vibrational level $\mathrm{v}^{\prime \prime}=0$ absorbs a photon of light from a frequency doubled tunable dye laser at about $282 \mathrm{~nm}$ to go to the first excited $\mathrm{A}^{2} \Sigma$ states in $\mathrm{v}^{\prime}=1$. In the excited electronic state $\mathrm{OH}$ has a very short lifetime of approximately 700 nanoseconds. ${ }^{31-33}$

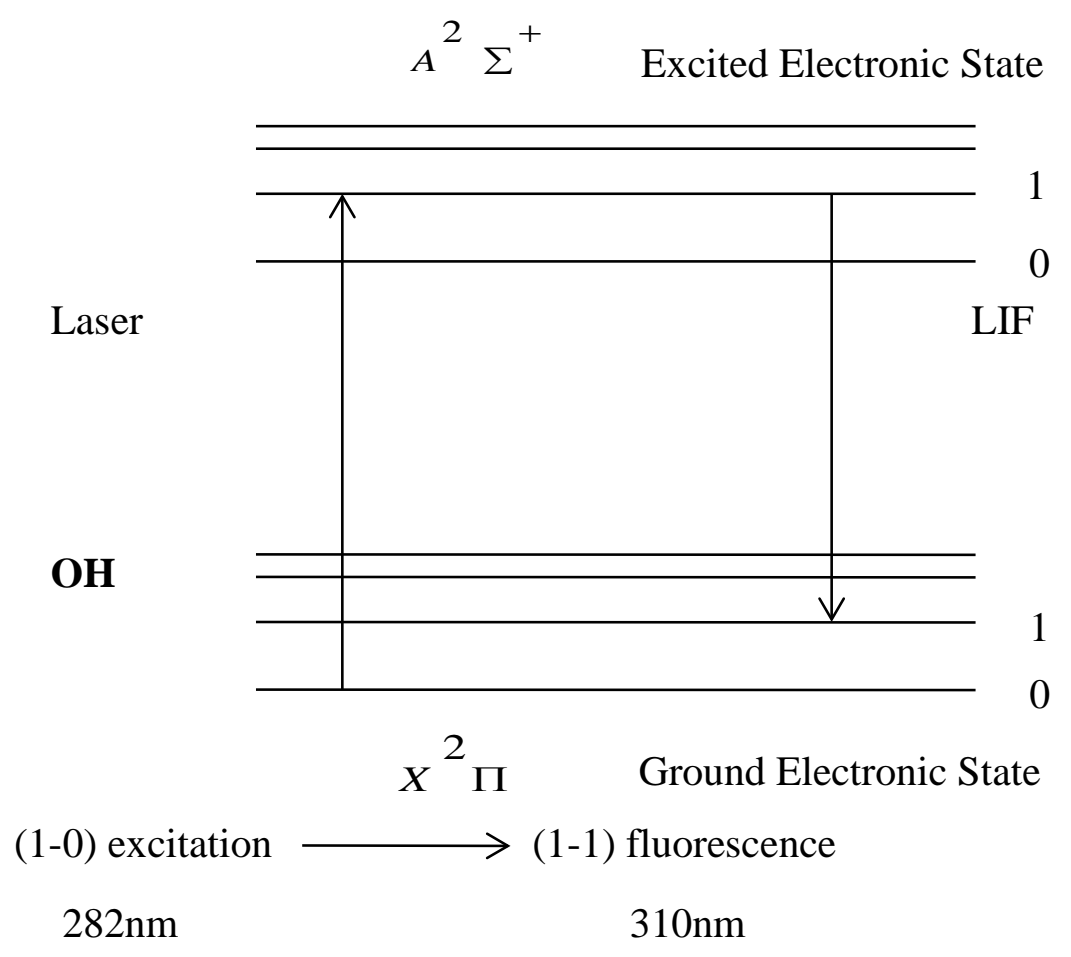

Figure 4. Schematic of laser induced fluorescence of Hydroxyl Radical

In figure 4, the ground and first excited vibrational states of each electronic states are labeled by the quantum numbers $v=0,1$. Only the fluorescence from the first vibrational level of 
the excited state to the first vibrational level of the ground electronic state at $(310 \mathrm{~nm})$ is observed using a band pass filter (FWHM $\pm 10 \mathrm{~nm})$.

Figure 5 shows the experimental LIF spectrum of $\mathrm{OH}$ radical for excitation wavelengths ranging from $281 \mathrm{~nm}$ to $283 \mathrm{~nm}$. All the rotational lines in Figure 5 are from the $A-X\left(v^{\prime}=1\right.$, $\mathrm{v}^{\prime \prime}=0$ ) vibronic band. The most intense absorption line at $281.95 \mathrm{~nm}$ corresponds to a superposition of several rotational lines and is used for the $\mathrm{OH}$ detection. The fluorescence emission is monitored at $310 \mathrm{~nm}$ using a band pass filter (FWHM $\pm 10 \mathrm{~nm}$ ).

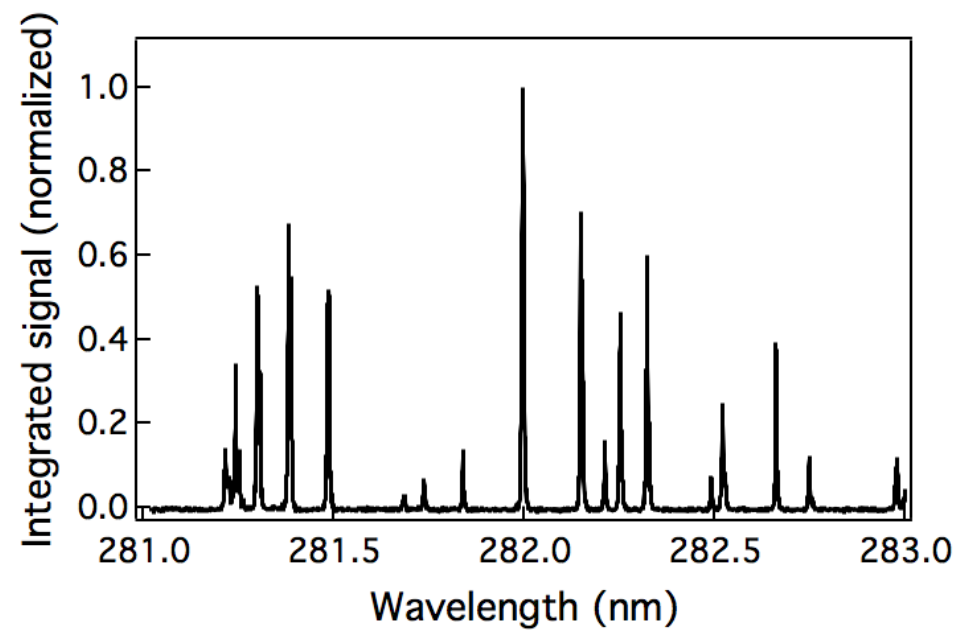

Figure 5. OH LIF spectrum for $A^{2} \Pi-X^{2} \Sigma$ electronic transition $\left(v^{\prime}=1, v^{\prime \prime}=0\right)$

In the equations below, $\mathrm{OH}$ refers to the ground electronic state of the hydroxyl radical while $\mathrm{OH}^{*}$ refers to the excited electronic state. The coefficient $\mathrm{k}^{*}$ is the first order decay rate of the excited state and is related to the Einstein $A$ coefficient for spontaneous emission and nonradiative quenching rate. It corresponds to the inverse of the fluorescence lifetime. Within a steady state approximation the fluorescence signal is directly proportional to the concentration of ground state $\mathrm{OH}$ radicals.

$\mathrm{OH}+\mathrm{h} v \rightarrow \mathrm{OH}^{*}$

$\mathrm{OH}^{*} \stackrel{\mathrm{k}^{*}}{\longrightarrow} \mathrm{OH}+\mathrm{h} v$ 
$[\mathrm{LIF}] \propto\left[\mathrm{OH}^{*}\right] \propto[\mathrm{OH}]$

Figure 6 shows the typical fluorescence decay following the dye laser excitation. The $Y$ axis represents the laser-induced fluorescence and the $X$-axis represents fluorescence time in nanoseconds. The laser triggers at 50ns and the fluorescence decay is gated using an integration window from a boxcar average. As shown in the figure 6, the fluorescence decay is in the order of nanoseconds, which is much shorter than the reaction time, which occurs in the order of microseconds.

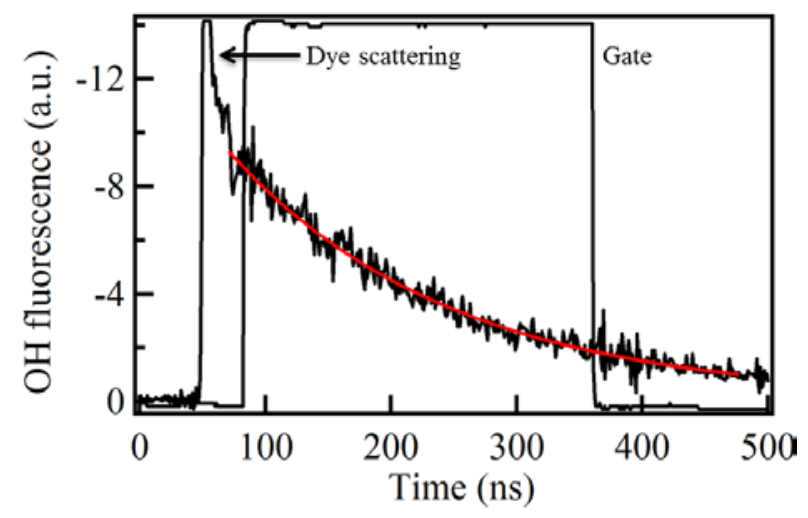

Figure 6. Fluorescence decay due to the dye laser

The main advantages of using LIF for the detection of $\mathrm{OH}$ radicals are:

1. LIF is very sensitive time resolved and radical specific technique.

2. It is a spatially resolved measurement technique.

The limitations of LIF are:

1. The technique measures the ground state population indirectly making it difficult to quantify due to quenching of the excited state. The concentration of $\mathrm{OH}$ ground state is monitored by looking at the fluorescence signal emitted from the $\mathrm{OH}$ excited state. The 
fluorescence signal is assumed to be proportional to the amount of radical in the excited state, which in turn is assumed to be proportional to the ground state $\mathrm{OH}$ radicals. Thus, LIF is an indirect measurement technique.

2. Not all species (radicals or molecules) absorb at the desired wavelengths and fluoresce.

Table 1 shows some of the widely studied radicals using laser-induced fluorescence. It shows the excitation wavelengths and detection wavelengths corresponding to their transitions. The radicals are detected using band pass or long pass filters.

Table 1. Excitation and detection wavelengths of the radicals corresponding to their transitions

\begin{tabular}{cccc}
\hline Radical & Excitation wavelength & Transition & Detection range \\
\hline $\mathrm{OH}^{34}$ & 282 & $\mathrm{~A}-\mathrm{X}$ & 310 (band-pass filter) \\
$\mathrm{CH}^{35}$ & 431 & $\mathrm{~A}-\mathrm{X}$ & 490 (band-pass filter) \\
$\mathrm{CN}^{36}$ & 387 & $\mathrm{~B}-\mathrm{X}$ & 420 (band-pass filter) \\
${ }^{1} \mathrm{CH}_{2}{ }^{37}$ & 537 & $\mathrm{~B}-\mathrm{A}$ & 580 (band-pass filter) \\
$\mathrm{HCO}^{38}$ & 258.7 & $\mathrm{~B}-\mathrm{X}$ & 300 (band-pass filter) \\
$\mathrm{H}_{2} \mathrm{CO}^{39}$ & 370 & $\mathrm{~A}-\mathrm{X} 4_{1}{ }^{0}$ & $>380$ (long-pass filter) \\
\hline
\end{tabular}

\subsection{Laser and Optical Systems}

Laser is an acronym for 'light amplification by stimulated emission of radiation'. A laser consists of three main elements: pumping source, active or gain medium, and a laser cavity. Laser actions occur through stimulated emission when the population of the upper energy level of the active medium is much higher than that of the lower energy level, referred to as population inversion. Active medium may be solid (e.g. Nd:YAG laser), liquid (e.g. dye laser) or gas (e.g. excimer laser). 
Stimulated emission is the emission of a photon upon interaction of the excited medium with a resonant photon. It follows: $M^{*}+h c \bar{v} \rightarrow M+2 h c \bar{v}$ where $M^{*}$ is the excited electronic state of the active medium, $h$ is Planck's constant, $c$ is the speed of light, $\bar{v}$ is the wave number and $\mathrm{M}$ is the ground state of the active medium. Figure 7 displays a schematic of a laser. The laser cavity is made of two highly reflecting mirrors, one on the left which is totally reflecting, coated with alternate layers of high dielectric substance (e.g. $\mathrm{TiO}_{2}$ ) and low dielectric substance (e.g. $\mathrm{SiO}$ ). The other mirror allows $1-10 \%$ of the laser beam to exit the cavity. The cavity contains the active medium in the middle, which is pumped by the pumping source. The photons generated through spontaneous emission initially strike the mirrors perpendicularly increasing the photon flux and causing the stimulated emission.

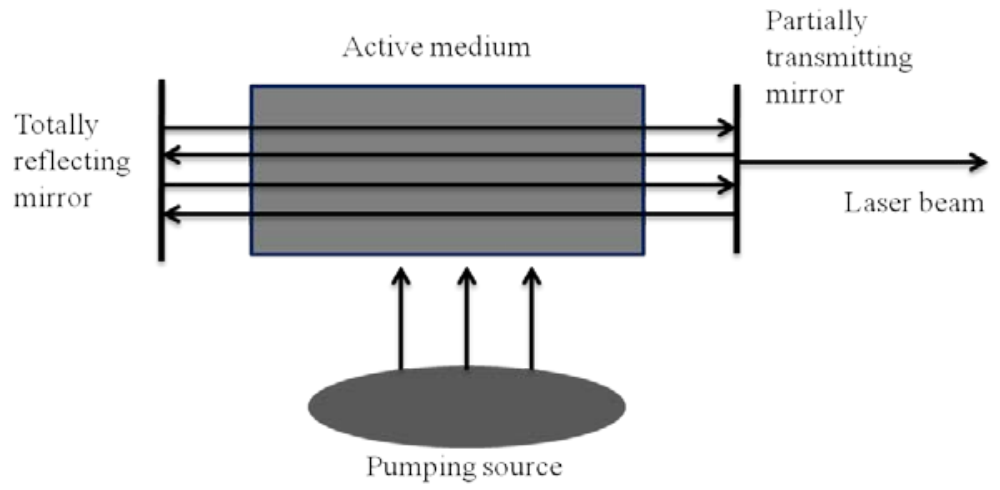

Figure 7. Schematic of a laser cavity

Population inversion is achieved by exciting the active medium using the pumping energy. In the case of a two-level laser system, it is not possible to achieve population inversion through light absorption. Excimer lasers are an example of two-level laser system in which the population of the upper state is created by an electrical discharge through atomic collisions. The more important are the noble gas halide gas lasers that have repulsive ground states but bound 
excited states often called exciplex. An exciplex is a diatomic molecule of two different atoms, which is stable in its excited electronic state but unstable or dissociates in its ground state e.g. ArF, $\mathrm{KrF}, \mathrm{XeF}$, etc. At thermal equilibrium the population in level 2 is always less than in level 1. Upon pumping and after reaching a steady state between absorption and stimulated emission the maximum population of level 2 is equal to that of level 1.

Figure 8 (a) and (b) displays a schematic of three-level and four-level lasing systems respectively. In the case of a three-level lasing system, after pumping from level 1 to 2, a fast decay occurs from 2 to 3 leading to population inversion between levels 3 and 1 . The lasing is observed by transition from 3 to 1 . In a four-level lasing system, upon pumping from level 1 to 2 , a fast non-radiative decay leads to population inversion between levels 3 and 4 . The lasing occurs by transition from levels 3 to 4 . Fast decay from level 4 to 1 makes the population inversion more efficient.
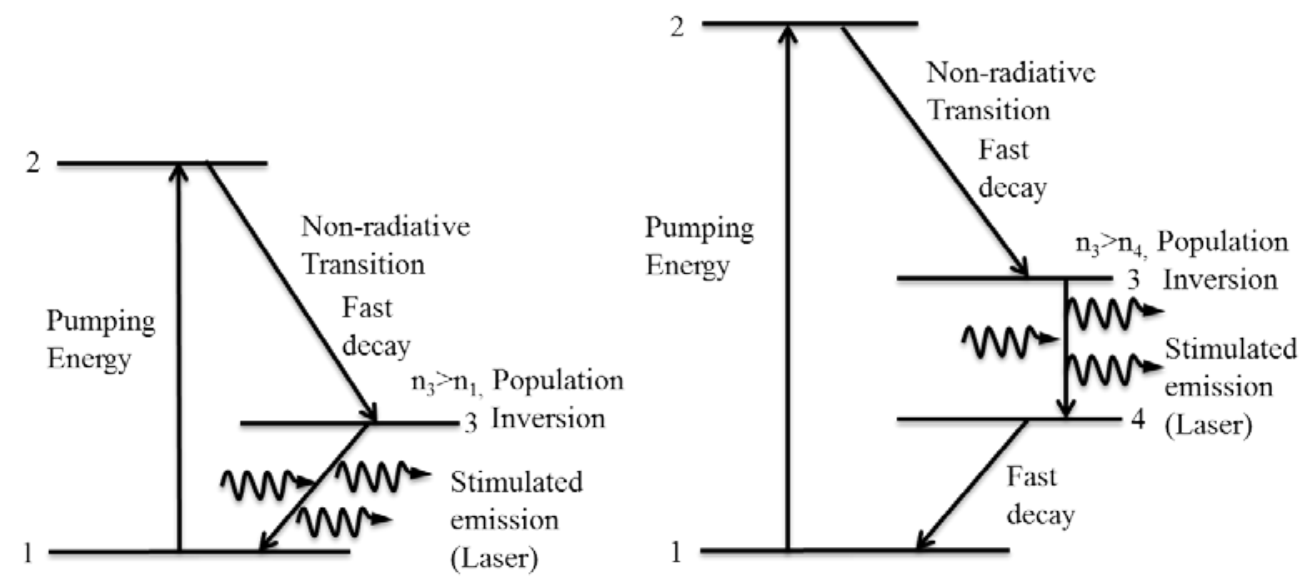

Figure 8 (a) Three-level and (b) Four-level lasing systems

A laser beam is unique because of its directional nature i.e. produces highly parallel beam, monochromatic (i.e. generates a very narrow wavelength range), extremely bright (i.e. 
power emitted per unit area per unit solid angle is very high), and coherent nature (i.e. electromagnetic waves are in phase).

\subsubsection{Nd:YAG and Harmonic Generation}

The neodymium-yttrium aluminium garnet (Nd:YAG) is a solid state laser that has embedded $\mathrm{Nd}^{3+}$ ions in a solid matrix (a synthetic mineral) called yttrium aluminium garnet $\left(\mathrm{Y}_{3} \mathrm{Al}_{5} \mathrm{O}_{12}\right)$. The $\mathrm{Nd}^{3+}: \mathrm{YAG}$ rod is 6-9 $\mathrm{cm}$ long and has $0.5-2.0 \%$ by weight of $\mathrm{Nd}^{3+}$ ions. ${ }^{40}$ The ground configuration of $\mathrm{Nd}^{3+}$ is $\mathrm{KLM} 4 \mathrm{~d}^{10} 4 \mathrm{f}^{3} 5 \mathrm{~s}^{2} 5 \mathrm{p}^{6}$ and the important terms arising from this configuration are ${ }^{4} \mathrm{I}$ and ${ }^{4} \mathrm{~F}$. Figure 9(a) shows the energy levels of multiplets arising from ${ }^{4} \mathrm{I}$ and ${ }^{4} \mathrm{~F}$ terms of free $\mathrm{Nd}^{3+}$ ions. Figure 9 (b) shows the ${ }^{4} \mathrm{~F}_{3 / 2}$ state split into two components due to crystal field interactions and ${ }^{4} \mathrm{I}_{11 / 2}$ splits into six components. The laser action involves the transition of ${ }^{4} \mathrm{~F}_{3 / 2}{ }^{4} \mathrm{I}_{11 / 2}$ that occurs at $1.064 \mu \mathrm{m}$. It can be seen in the figure that ${ }^{4} \mathrm{~F}_{3 / 2}$ is the lowest energy of ${ }^{4} \mathrm{~F}$ term while ${ }^{4} \mathrm{I}_{11 / 2}$ is not the lowest energy state. This indicates that Nd:YAG is a fourlevel laser. The transition that is important for laser has $\Delta \tilde{v}$ (wave number) $=9391 \mathrm{~cm}^{-1}$ that corresponds to $\Delta \lambda$ (wavelength) $=1064 \mathrm{~nm}$ at room temperature. 


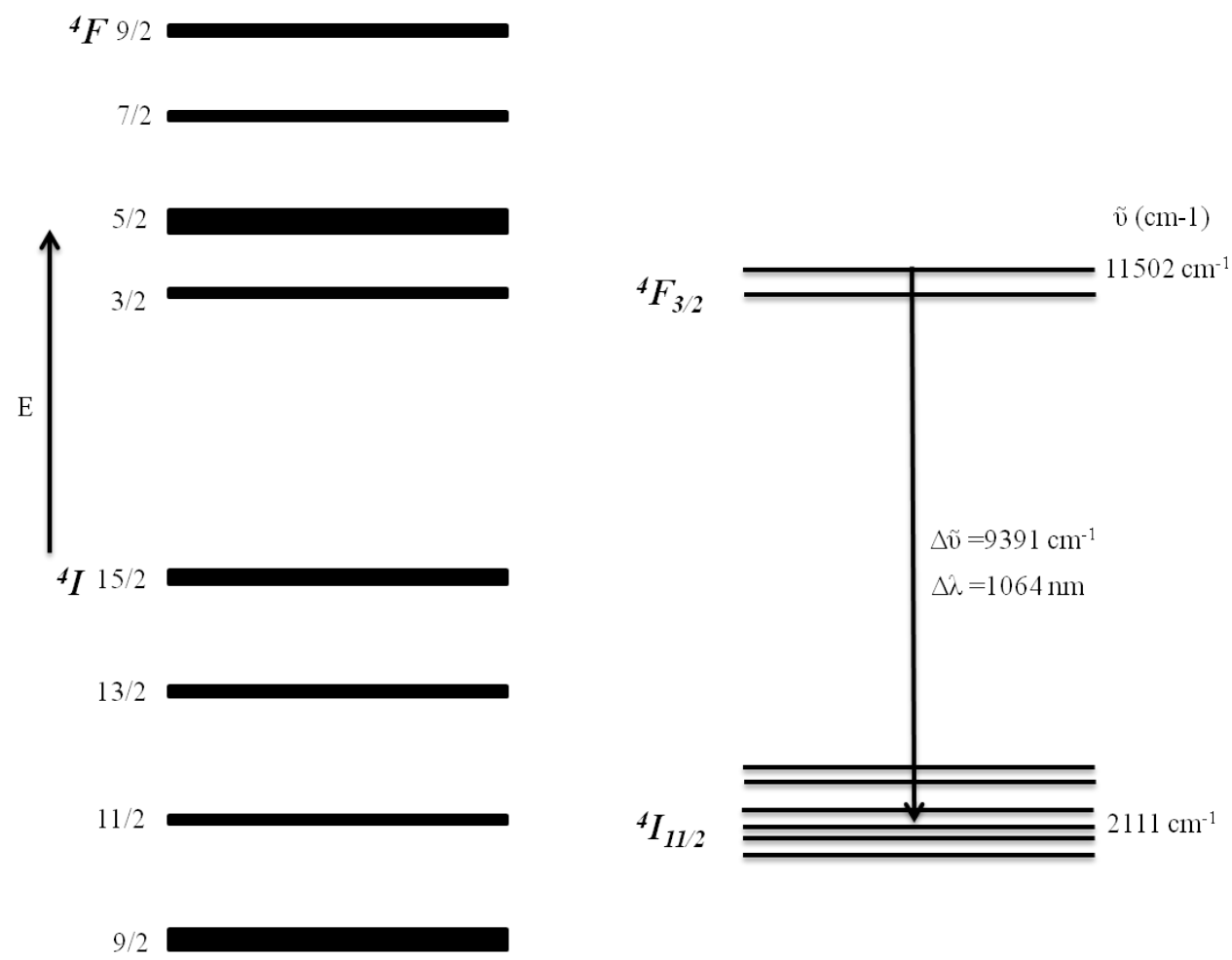

Figure 9. Energy levels in (a) Free $\mathrm{Nd}^{3+}$ and (b) $\mathrm{Nd}^{3+}$ split by crystal field interactions ${ }^{40}$

Nd:YAG laser that operates at fundamental wavelength of 1064 can be frequency doubled, tripled, and quadrupled to obtain wavelengths of $532 \mathrm{~nm}, 355 \mathrm{~nm}$, and $266 \mathrm{~nm}$ respectively. This can be achieved by harmonic generation.

\subsubsection{Harmonic Generation ${ }^{40}$}

The relation between the oscillating electric field (E) of light propagating in a medium with the induced dipole moment $(\mu)$ and polarizability $(\alpha)$ is given by equation 7 .

$\mu=\alpha \mathrm{E}+\frac{1}{2} \beta \mathrm{E} \cdot \mathrm{E}+\frac{1}{6} \gamma \mathrm{E} \cdot \mathrm{E} \cdot \mathrm{E}+\frac{1}{24} \delta \mathrm{E} \cdot \mathrm{E} \cdot \mathrm{E} \cdot \mathrm{E}+\ldots \ldots .$.

where $\beta$ is the hyperpolarizability, $\gamma$ the second hyperpolarizability, and $\delta$ the third hyperpolarizability. These second and third terms are non-linear in E. Because of high laser 
power these terms become significant and lead to harmonic generation. The magnitude of the oscillating electric field can be given by equation 8 .

$\mathrm{E}=\mathrm{A} \sin 2 \pi v \mathrm{t}$

where $\mathrm{A}$ is the amplitude and $v$ the frequency. The square of the electric field in equation 8 can therefore be written as

$E^{2}=A^{2}(\sin 2 \pi v t)^{2}=\frac{1}{2} A^{2}(1-\cos 2 \pi 2 v t)$

The cosine term in equation 9 has twice the frequency than the fundamental radiation. This process of frequency doubling is called second harmonic generation. Although fourth harmonics can be obtained directly from the $3^{\text {rd }}$ and $4^{\text {th }}$ term of equation 7 , higher conversion is obtained by doubling the $2^{\text {nd }}$ harmonic with a second crystal. Third harmonic generation is obtained by frequency mixing of the fundamental at $1064 \mathrm{~nm}$ with the second harmonic at 532 following a similar mechanism.

To achieve the second, third and fourth harmonic generations experimentally, various crystalline materials are commercially available. Some of the examples include potassium pentaborate $\left(\mathrm{KB}_{5} \mathrm{O}_{8}, \mathrm{KPB}\right), \beta$-barium borate $\left(\mathrm{BaB}_{2} \mathrm{O}_{4}, \mathrm{BBO}\right)$, and lithium niobate $\left(\mathrm{Li}_{3} \mathrm{NbO}_{4}\right)$ that are suitable for the limited wavelength range. In the present experiments BBO crystals are used. The efficiency of frequency doubling is 20 to $30 \%$.

\subsubsection{Q-Switching ${ }^{40}$}

The quality factor or the resolving power of the laser cavity is defined by equation 10 . 
$\mathrm{Q}=\frac{\mathrm{v}}{\Delta \mathrm{v}}$

Eq. (10)

where $v$ is the frequency and $\Delta v$ is the line width of the laser radiation. The relation of $\mathrm{Q}$ with the energy stored in the cavity $\left(E_{c}\right)$ and the energy allowed to leak out $\left(E_{t}\right)$ during a time $t$ is given by the equation 11.

$\mathrm{Q}=\frac{2 \pi \nu \mathrm{E}_{\mathrm{c}} \mathrm{t}}{\mathrm{E}_{\mathrm{t}}}$

In Q-switching operation, the $Q$ of a laser cavity is reduced for a very short period of time. This prevents the oscillation of laser radiation between the cavity mirrors. During this time the population inversion in the upper level increases. Then $Q$ is allowed to increase rapidly resulting in very short pulses. This cycle is repeated.

In this research, the third and fourth harmonic of a Continuum Surelite III-10 Nd:YAG (10 Hz) laser were used to photolyze $\mathrm{HONO}$ at $355 \mathrm{~nm}$ and $\mathrm{H}_{2} \mathrm{O}_{2}$ at $266 \mathrm{~nm}$, respectively. The photolysis beam utilized had an average diameter of $\sim 7.5 \mathrm{~mm}$ with maximum measured laser fluence at the entrance of the reaction cell of $147 \mathrm{~mJ} / \mathrm{cm}^{2}$. The second harmonic of a Nd:YAG (532 nm) was used to pump a dye laser. Table 2 shows the specifications that includes beam

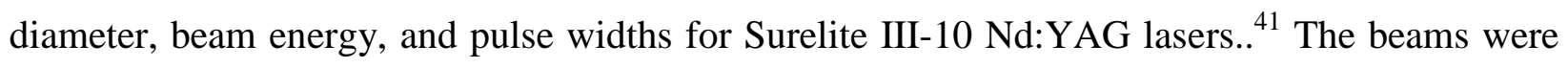
horizontally polarized at 1064, 355 and $266 \mathrm{~nm}$ and vertically polarized at $532 \mathrm{~nm}$. 
Table 2. Specifications of Nd:YAG laser

\begin{tabular}{ll}
\hline Beam diameter & $9.5 \mathrm{~mm}$ \\
\hline Energy & \\
\hline $1064 \mathrm{~nm}$ & $850 \mathrm{~mJ}$ \\
$532 \mathrm{~nm}$ & $425 \mathrm{~mJ}$ \\
$355 \mathrm{~nm}$ & $165 / 225 \mathrm{~mJ}$ \\
$266 \mathrm{~nm}$ & $100 \mathrm{~mJ}$ \\
\hline Pulse width & \\
\hline $1064 \mathrm{~nm}$ & $4-6 \mathrm{~ns}$ \\
$532 \mathrm{~nm}$ & $3-5 \mathrm{~ns}$ \\
$355 \mathrm{~nm}$ & $3-5 \mathrm{~ns}$ \\
$266 \mathrm{~nm}$ & $3-5 \mathrm{~ns}$ \\
\hline
\end{tabular}

Figure 10 shows the plot of intensity of Nd:YAG laser versus time in nanoseconds. The pulse width of Nd:YAG laser at $1064 \mathrm{~nm}$ is approximately 6 ns and is very shorter than the reaction time.

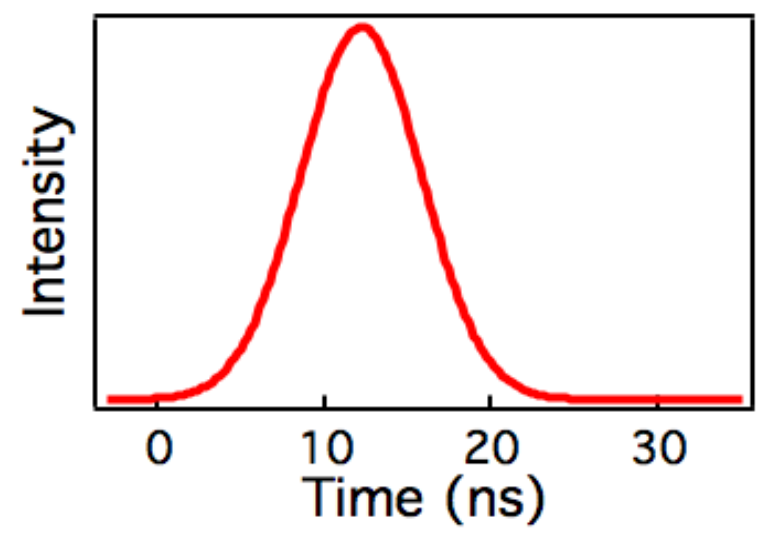

Figure 10. Nd:YAG laser profile at $1064 \mathrm{~nm}$

\subsubsection{Tunable Dye Laser}

A dye laser is tunable over a wide range of wavelength and has an organic liquid dye as active medium. These dyes have strong visible absorptions from the ground electronic state (S0) to the first excited singlet state (S1). The dye has oscillator strengths (related to the probability of 
transition) nearly equal to $1^{42,43}$ and fluorescence quantum yield (number of molecules fluorescing/number of quanta absorbed) nearly equal to $1 .{ }^{44}$ In the present research, Rhodamine 590 (Rhodamine 6G) dissolved in methanol is used.

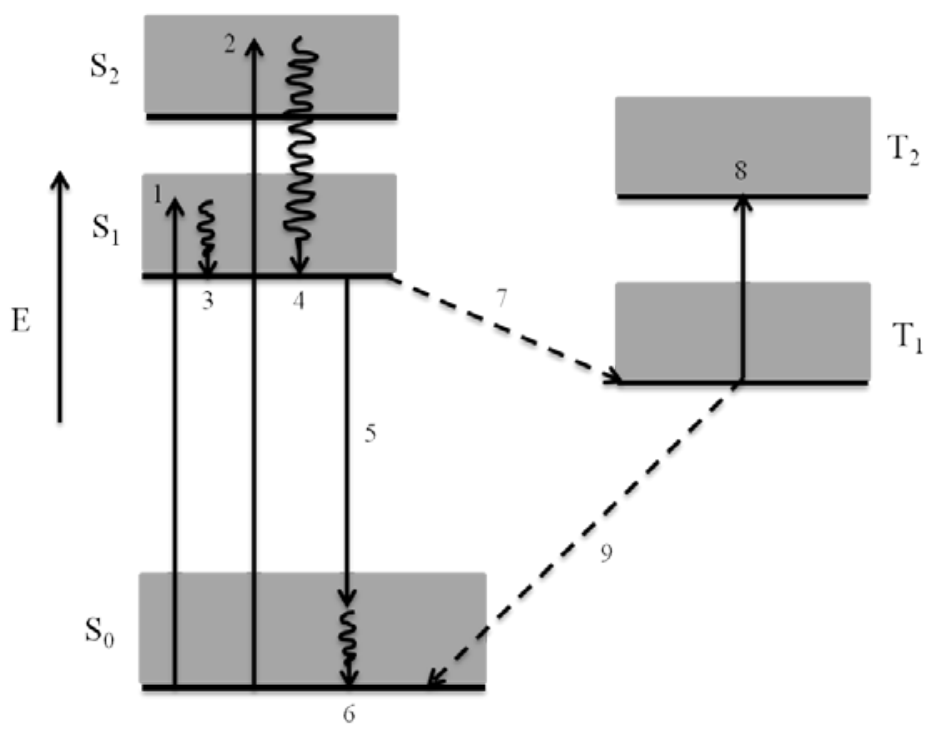

Figure 11. Energy level scheme for a dye molecule nine processes important in laser action $^{40}$

Figure 11 shows the energy level diagram for a dye molecule. $S_{0}, S_{1}$, and $S_{2}$ are the lowest electronic singlet states whereas $T_{1}$ and $T_{2}$ are the triplet states. These states comprise vibrational and rotational sub-levels that appear as continuum due to interaction with the liquid solvent. The population inversion can be achieved in $S_{1}$ or $S_{2}$ as shown in the Figure 11 by 1 and 2 absorption. Here the collisional or vibrational-rotational relaxation occurs by processes labeled 3 and 4 within 10 picoseconds (ps). Lasing occurs between the bottom of the S1 band and the S0 band and from there relaxation process occurs (6) to the bottom of the band. The population of $S_{1}$ can also decay due to process 7 (intersystem crossing). The lifetime of molecules in $T_{1}$ is long 
ranging from $100 \mathrm{~ns}$ to $1 \mathrm{~ms}$, due to the forbidden $\mathrm{T}_{1}-\mathrm{S}_{1}$ transition (process 9) therefore building up the concentration of molecules. The spin allowed transition $\mathrm{T}_{1}-\mathrm{T}_{2}$ can occur even by the fluorescence from 5 and can decrease the efficiency of laser. This can be prevented by pulsed pumping method allowing enough time for $\mathrm{S}_{1}-\mathrm{S}_{0}$ relaxation.

Figure 12 shows the structure of the Rhodamine 590 (Rhodamine 6G) dye that was used in this research, with a lasing maximum at $560 \mathrm{~nm}^{45}$ The $\mathrm{X}^{-}$is $\mathrm{ClO}_{4}^{-}$. A solution in methanol was used for the oscillator and amplifier. The concentration of dye in methanol used in the oscillator was $5.25 \times 10^{-5} \mathrm{M}$ and amplifier was $7.77 \times 10^{-5} \mathrm{M}$. The oscillator operates through population inversion within the optical cavity. The Amplifier operates through population inversion without optical oscillation. This means that the beam passes once and the amplifier cavities amplify the beam power.

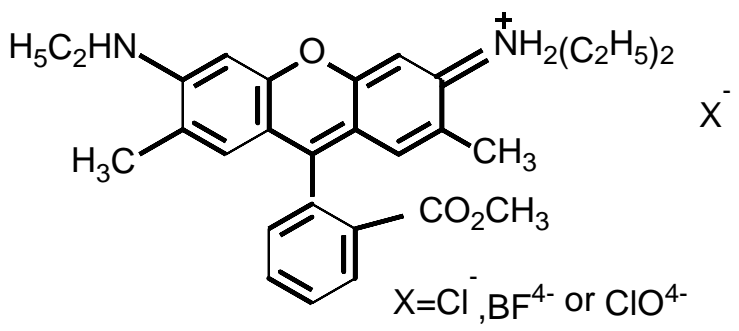

Figure 12. Structure of Rhodamine 590 dye

Table 3 shows the specifications that includes energy, beam diameter, polarization, tuning range, etc. for the dye laser pumped by second harmonic of Nd:YAG laser. ${ }^{46}$ 
Table 3. Specifications of tunable dye laser

\begin{tabular}{ll}
\hline Fundamental wavelength $(560 \mathrm{~nm})$ & $300 \mathrm{~mJ} / \mathrm{pulse}$ \\
Energy & $380-980 \mathrm{~nm}$ \\
Tuning range & $3-6 \mathrm{~mm}$ \\
Beam diameter & $>30 \%$ \\
Efficiency & \\
Pump energy & $100-1000 \mathrm{~mJ}$ \\
$532 \mathrm{~nm}$ & $85-400 \mathrm{~mJ}$ \\
$355 \mathrm{~nm}$ & \\
Polarization & vertical \\
Output with $532 \mathrm{~nm}$ pumping & vertical \\
Output with $355 \mathrm{~nm}$ pumping & \\
With high resolution single grating & $380-590 \mathrm{~nm}$ \\
Tuning range in the UV region & $206-980 \mathrm{~nm}$ \\
Tuning range with wavelength extension &
\end{tabular}

\subsubsection{Photomultilier Tube (PMT)}

A photomultiplier tube is a photo-sensitive device or photodetector that has extremely high sensitivity (low-light-level detection). A photomultiplier tube consists of a photocathode, focusing electrodes, dynodes or electron multipliers, and an electron collector or anode in a vacuum tube. It works by external photoelectric effect and provides ultra-fast response. As light strikes the photocathode, it emits photoelectrons. These electrons are focused using set of electrodes and multiplied by dynodes or electron multipliers through secondary electronemission. The multiplied photoelectrons are collected by an anode or electron collector. The secondary emission electron multiplication is responsible for the extremely high sensitivity. The photomultiplier tube has a very fast time response, very low noise and works in UV, visible, and near IR regions. ${ }^{47,48}$

The photomultiplier tube used in this research had a side-on configuration (R928, Hamamatsu) and was operated at 500-850 V. Side-on configuration implies that the 
photocathode lies on the side and receives light unlike head-on configuration where photocathode lies on top and receives light from the top part. A side-on PMT has a reflection mode photocathode or opaque photocathode reflecting the ejected photoelectrons to the first dynode. A head-on PMT operates according to a transmission mode (semi-transparent) photocathode which transmits the ejected photoelectrons in to the vacuum. ${ }^{47}$ Figure 13 shows the opaque or reflection mode photocathode in a circular cage used in side-on PMT. When light strikes the photocathode, photoelectrons make their way to the first dynode.

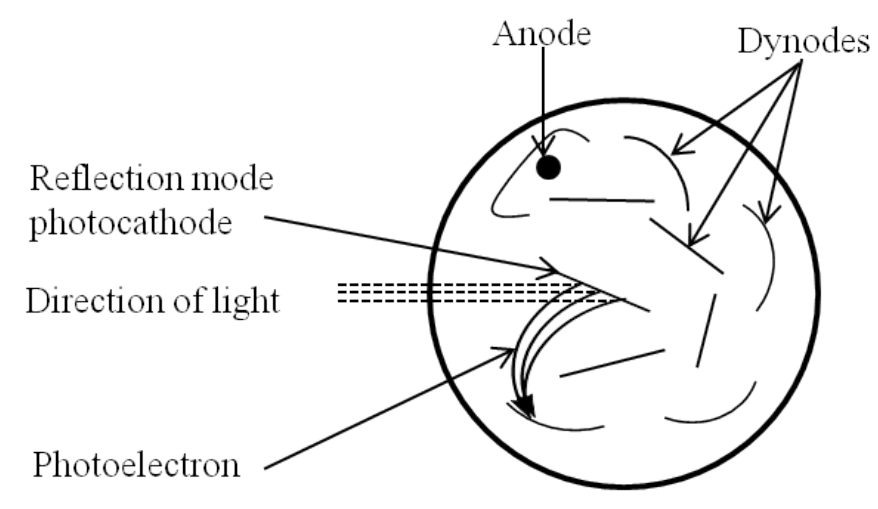

Figure 13. Reflection mode photocathode in a circular cage in side-on photomultiplier tube $^{47}$

In a side-on configuration, the PMT has a circular cage multiplier resulting in high sensitivity (cathode sensitivity ranges from 18-3.5 mA/W at 194-852 $\mathrm{nm}$ and anode sensitivity ranges from $1.8 \times 10^{5}-3.5 \times 10^{4} \mathrm{~A} / \mathrm{W}$ at $194-852 \mathrm{~nm}$ ) and amplification at low voltage. ${ }^{47}$ Circular cage multiplier is compact and provides fast time response (anode pulse time rise is $2.2 \mathrm{~ns}$ ) but are limited by dark currents. ${ }^{47}$ Dark current is produced even if the PMT is operated in completely dark place. One of the main reasons for dark current is the thermionic emission from photocathode and dynodes due to their very low work functions. This can be avoided by decreasing the temperature. ${ }^{47}$ 
The photocathode in a side-on configuration is made of alkali metals of low work functions. The multialkali (Na-K-Sb-Cs) photocathode results in wide spectral response ranging from UV to near IR region (185-900 nm). The window placed before the photocathode acts as the wavelength filter that transmits UV and visible light up to $185 \mathrm{~nm}^{48,49}$

\subsection{Kinetic Measurements}

\subsubsection{LIF Measurements}

The reactions of hydroxyl radicals with polycyclic aromatic hydrocarbons were studied by laser spectroscopy using two kinds of lasers (Nd:YAG and tunable dye). The output of laser beam from the Nd:YAG was directed to the reaction chamber using highly reflecting mirrors of $25 \mathrm{~mm}$ diameter and $5 \mathrm{~mm}$ thickness. For $355 \mathrm{~nm}$, NB1-K08 mirrors from Thorlabs were used whereas for 266 nm NB1-K04 mirrors were used. The frequency doubled dye laser beam (282 $\mathrm{nm}$ ) was directed to the reaction chamber using UV right angle prism of $20 \mathrm{~mm}$ diameter (Thorlabs PS608). The fluorescence from the hydroxyl radicals was detected using the photomultiplier tube (Hamamtsu R928) orthogonal to the laser beams. Before the PMT, two uncoated fused silica plano convex lenses of diameter $25.4 \mathrm{~mm}$ and focal length of $25 \mathrm{~mm}$ (LA4663) from Thorlabs were used to image the fluorescence signal to the PMT photocatode. In addition, a bandpass filter at $310 \mathrm{~nm}$ (fwhm $\pm 10 \mathrm{~nm}$ ) was placed just before the PMT to collect fluorescence signal.

The kinetic measurements of the reaction of the hydroxyl radicals with polycyclic aromatic hydrocarbons occur in the microsecond time-scale. The time available for reaction was varied by delaying the probe laser relative to the pump laser. The timing scheme and the details of the kinetic measurements are discussed in the following sub-sections. 


\subsubsection{Pump-Probe Timing}

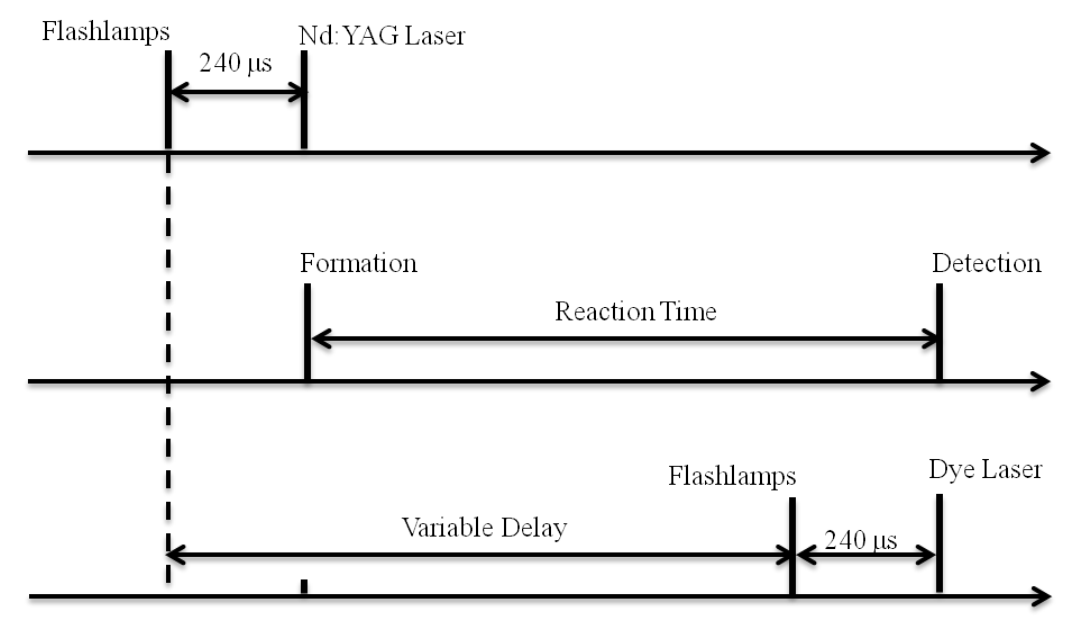

Figure 14. Timing between the pump and probe lasers

Figure 14 shows the timing of the pump and probe lasers employed to measure the kinetics of the bimolecular reactions. The pump and probe lasers trigger $240 \mu \mathrm{s}$ after the flashlamps lamp (Q-switch time). The triggering of the pump (Nd:YAG) laser corresponds to the formation of the hydroxyl radicals whereas the triggering of probe (dye) laser corresponds to their detection. The timing is controlled by a delay generator (DG 535) with an internal time trigger reference. In the experiments, the trigger of the dye laser flash lamp was fixed relatively to the trigger origin. The trigger of the Nd:YAG pump laser was varied relative to the dye laser flash lamp trigger. A negative delay time corresponded to the Nd:YAG laser firing before the dye laser. A positive delay corresponded to the dye laser firing first. The available time for the reaction to occur was therefore the opposite of the laser delay-time. During the experiments, the probe laser was initially set to trigger before the pump laser to observe the baseline. The data were usually taken for reaction times ranging from $-100 \mu$ s to $500 \mu \mathrm{s}$. Reaction times between 100 to $0 \mu$ s corresponded to baseline, as no radicals were generated before the probe laser. At 0 
$\mu \mathrm{s}$, the pump and probe lasers were triggering at the same time. Signal was detected after $0 \mu \mathrm{s}$, for which the probe laser fired after the pump laser. The pump laser profile and fluorescence decay of the radical were in the order of nanoseconds while the reaction was in the order of microseconds. Therefore, the reaction was not affected by the laser profile and fluorescence decay.

\subsubsection{Example of LIF Emission}

Figure 15 shows the average LIF signal of the $\mathrm{OH}$ radical recorded by excitation at 282 $\mathrm{nm}$ and emission at $310 \mathrm{~nm}$ against time in nanoseconds. The red line is the fit to the fluorescence data and blue line is the time gate over which the signal is integrated by a boxcar averager. The width is set to $280 \mathrm{~ns}$ to include most of the signal while avoiding laser scattering and noise. The crossed area under the signal represents the magnitude of the fluorescence signal.

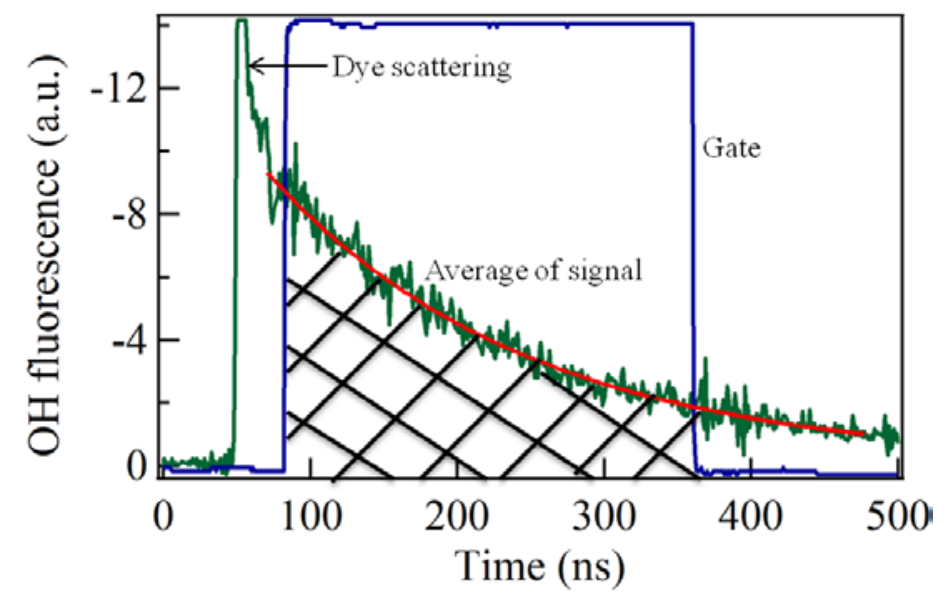

Figure 15. Emission of OH fluorescence following dye laser excitation

The exponential fit of the fluorescence decay in figure 16 using the equation 12 gives the lifetime $\tau$ of the $\mathrm{OH}$ radical. 
$[\mathrm{A}]=[\mathrm{A}]_{0} \mathrm{e}^{-\frac{\mathrm{t}}{\tau}}$

Eq. (12)

$[\mathrm{A}]_{0}$ is the concentration of radical in the ground electronic state. The fit in Figure 15 yields a lifetime for the $\mathrm{OH}$ radical of 179 ns.

After getting the optimum $\mathrm{OH}$ fluorescence signal, the radical number density temporal profiles were obtained by averaging 20 laser shots per point while changing the delay time between the lasers using a delay generator (Stanford research systems, DG535). A GPIB interface paired to a PC running LabView was used to average, store and analyze the data for the reaction of $\mathrm{OH}$ radical with propene, butene, acetaldehyde, and benzene.

\subsubsection{Pseudo First Order Approximation}

The reaction of hydroxyl radical with polycyclic aromatic hydrocarbon precursor is overall a second order reaction. To avoid the complexity of measuring the reactant concentrations of both species simultaneously, the experiments were performed under pseudofirst order approximation. In this case, one of the reactants is introduced in large excess and can be assumed constant relative to the concentration of the other reactant. Hydroxyl radical reacts with the gaseous reactant (A) to give products with a second order rate coefficient $k$ which is to be determined. Hydroxyl radicals also react with other molecules such as its precursor or with other $\mathrm{OH}$ radicals to give products with rate coefficient $k^{\prime}$ as shown below.

$\mathrm{OH}+\mathrm{A} \stackrel{\mathrm{k}}{\longrightarrow}$ Products

Other molecules $+\mathrm{OH} \stackrel{\mathrm{k}^{\prime}}{\longrightarrow}$ Products 
The rate law equation of the above two reactions R3 and R4 can be written as

$\frac{\mathrm{d}[\mathrm{OH}]}{\mathrm{dt}}=-\mathrm{k}[\mathrm{OH}][\mathrm{A}]-\mathrm{k}^{\prime}[$ Other molecules $][\mathrm{OH}]$

Eq. (13)

$\frac{\mathrm{d}[\mathrm{OH}]}{[\mathrm{OH}]}=-\left\{\mathrm{k}[\mathrm{A}]+\mathrm{k}^{\prime}[\right.$ Other molecules $\left.]\right\} \mathrm{dt}$

Applying pseudo first order approximation, the concentrations of the reactant A and other molecules are assumed to be independent on reaction time i.e. the concentrations of $\mathrm{A}$ and other molecules is very large compared to the concentration of hydroxyl radical.

[A] and [Other molecules] >> [OH]

Integrating the above rate law equation 14

$\int_{0}^{\mathrm{t}} \frac{\mathrm{d}[\mathrm{OH}]}{\mathrm{dt}}=-\left\{\mathrm{k}[\mathrm{A}]+\mathrm{k}^{\prime}[\right.$ Othermolecules $\left.]\right\} \int_{0}^{\mathrm{t}} \mathrm{dt}$

$\ln \frac{[\mathrm{OH}]}{[\mathrm{OH}]_{0}}=-\mathrm{k}_{1 \mathrm{st}} \mathrm{t}$

where $\mathrm{k}_{1 \mathrm{st}}=\left\{\mathrm{k}[\mathrm{A}]+\mathrm{k}^{\prime}[\right.$ Other molecules]

$\mathrm{k}_{1 \mathrm{st}}$ is the pseudo first order rate coefficient. The concentration of the $\mathrm{OH}$ radicals in the cell as a function of the delay between the two lasers $\Delta t$ can be written as:

$[\mathrm{OH}]_{\mathrm{t}}=[\mathrm{OH}]_{0} \mathrm{e}^{-\mathrm{k}_{1 \mathrm{st}} \Delta \mathrm{t}}$

The relative concentration of hydroxyl radical as a function of reaction time is obtained by plotting the LIF signal intensity as a function of laser delay time: 
$\mathrm{LIF}=\mathrm{LIF}_{0} \mathrm{e}^{-\mathrm{k}_{\mathrm{s}^{\mathrm{s}}} \Delta \mathrm{t}}$

The values of $\mathrm{k}_{1 \mathrm{st}}$ can then be determined by fitting the experimental data to an exponential decay. Experimentally, the temporal profiles of the relative $\mathrm{OH}$ concentration are recorded for multiple concentration of the co-reactant A.

The second order rate coefficient $k$ is determined by plotting the $k_{1 s t}$ values as a function of various number densities of the reactant (PAH precursor) and fitted to a straight line. The slope of this straight line gives the rate coefficient of the reaction of hydroxyl radical with the reactant. Figure 16 shows the $\mathrm{k}_{1 \mathrm{st}}$ vs the concentration of propene at 5 Torr and room temperature, one of the reactants used to validate the experimental set up. The slope of the linear fit is equal to the second-order rate coefficient $\left(\mathrm{k}_{2 n d}\right)$.

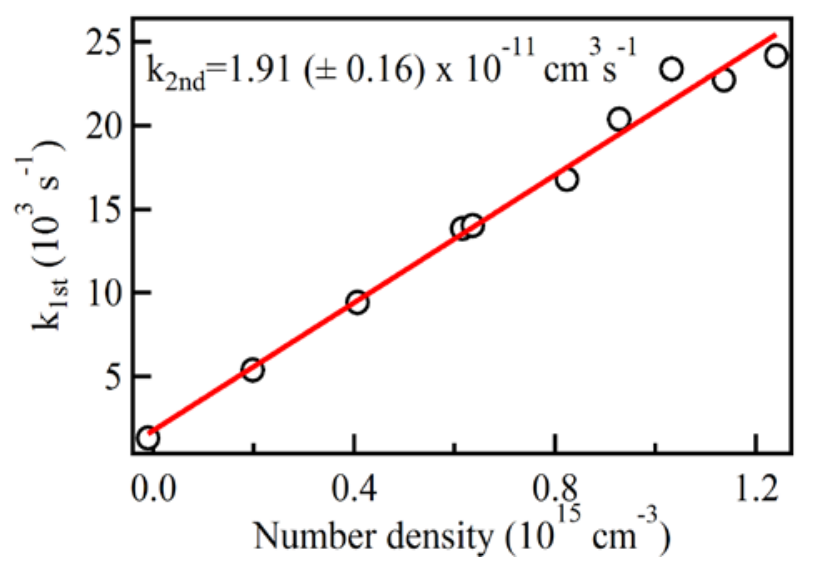

Figure 16. A plot of $k_{1 s t}$ vs number density of propene at 5 Torr. The line is obtained using a linear fit to the data and $\mathrm{k}_{2 n d}$ values are presented with $2 \sigma$ precision

\subsection{Experimental Validation}

The experimental set up to measure the kinetics of hydroxyl radicals with polycyclic aromatic hydrocarbon precursors in the laboratory was validated by measuring the reaction 
kinetics of hydroxyl radical with propene, ${ }^{50}$ butene, ${ }^{51}$ acetaldehyde, ${ }^{52}$ and benzene ${ }^{53}$ that are well known in the literature.

\subsubsection{OH + Propene, Butene, Acetaldehyde, and Benzene}

Validation of the experimental setup is achieved by measuring the rates of reaction of $\mathrm{OH}$ + propene $\left(\mathrm{C}_{3} \mathrm{H}_{6}\right)$, trans-2-butene $\left(\mathrm{C}_{4} \mathrm{H}_{8}\right)$, acetaldehyde $\left(\mathrm{CH}_{3} \mathrm{CHO}\right)$, and benzene $\left(\mathrm{C}_{6} \mathrm{H}_{6}\right)$. Propene and trans-2-butene were purchased from Matheson Tri Gas and used without further purification. Propene was regulated using Harris compressed gas regulator (model no. 25-15C) and trans-2butene was regulated using Matheson regulator (model no. 3320B) to inject into the reaction chamber. Benzene was purchased from Sigma Aldrich (HPLC grade, $>99.9 \%$ pure) and put inside the bubbler. It was carried to the reaction chamber using helium carrier gas. Acetaldehyde was purchased from Sigma Aldrich (anhydrous, GC grade $>99.9 \%$ pure). It was placed in the bubbler contained in ice-water bath. Then it was evaporated and collected in cylinders to make $5 \%, 10 \%$, and $15 \%$ mixture in helium buffer gas. The measured rates coefficients listed in Tables 4a and 4b agree well with published literature. ${ }^{52,54}$ The high temperature setup was tested and validated by measuring rates of reaction of $\mathrm{OH}+$ propene over a temperature range of 293-450 K. The observed inverse temperature dependence of the rate constant (see table $4 \mathrm{~b}$ ) is consistent with trends in published literature. ${ }^{55}$

Table 4a. Rate coefficients $\left(\mathrm{cm}^{3} \mathrm{~s}^{-1}\right)$ of the validation reactions compared to published literature

\begin{tabular}{lllll}
\hline \multicolumn{1}{c}{ Reaction } & \multicolumn{1}{c}{ This work } & \multicolumn{2}{c}{ Literature reviews } \\
\hline & Reaction rate & $\begin{array}{l}\text { Experimental } \\
\text { conditions }\end{array}$ & Reaction Rate & $\begin{array}{l}\text { Experimental } \\
\text { conditions }\end{array}$ \\
\hline $\mathrm{OH}+$ propene & $2.13( \pm 0.40) \cdot 10^{-11}$ & $\mathrm{~T}=296 \mathrm{~K}, \mathrm{P}=5 \mathrm{Torr}$ & $2.74 .10^{-1150}$ & $\mathrm{~T}=296 \mathrm{~K} ; \mathrm{P}=30 \mathrm{Torr}$ \\
\hline $\mathrm{OH}+$ butene & $5.2( \pm 0.11) \cdot 10^{-11}$ & $\mathrm{~T}=296 \mathrm{~K}, \mathrm{P}=5 \mathrm{Torr}$ & $6.99( \pm 0.70) .10^{-11}$ & $\mathrm{~T}=296 \mathrm{~K} ; \mathrm{P}=50 \mathrm{Torr}$ \\
& & & 51 & \\
\hline $\mathrm{OH}+$ acetaldehyde & $1.56( \pm 0.40) .10^{-11}$ & $\mathrm{~T}=296 \mathrm{~K}, \mathrm{P}=5$ Torr & $\begin{array}{l}1.52( \pm 0.15) .10^{-11} \\
52\end{array}$ & $\mathrm{~T}=296 \mathrm{~K} ; \mathrm{P}=100$ \\
\hline $\mathrm{OH}+$ benzene & $1.30( \pm 0.23) .10^{-12}$ & $\mathrm{~T}=296 \mathrm{~K}, \mathrm{P}=5$ Torr & $1.36( \pm 0.09) .10^{-12} 53$ & $\mathrm{~T}=296 \mathrm{~K} ; \mathrm{P}=20 \mathrm{Torr}$ \\
\hline
\end{tabular}


Table 4b. Rate coefficients ( $\mathrm{cm}^{3} \mathrm{~s}^{-1}$ ) for validation of the high temperature setup

\begin{tabular}{|c|c|c|c|c|}
\hline \multirow[t]{2}{*}{ Reaction } & \multicolumn{2}{|c|}{ This work } & \multicolumn{2}{|c|}{ Literature values $^{55}$} \\
\hline & Reaction rate $^{\mathrm{a}}$ & Temperature (K) & Reaction rate $^{b}$ & $\begin{array}{c}\text { Temperature } \\
\text { (K) }\end{array}$ \\
\hline \multirow{3}{*}{$\mathrm{OH}+$ propene } & $1.45( \pm 0.28) \cdot 10^{-11}$ & 300 & $1.87( \pm 1.5) \cdot 10^{-11}$ & 298 \\
\hline & $9.20( \pm 0.31) \cdot 10^{-12}$ & 393 & $7.8( \pm 0.3) \cdot 10^{-12}$ & 386 \\
\hline & $6.28( \pm 0.92) \cdot 10^{-12}$ & 423 & $5.05 .10^{-12}$ & 425 \\
\hline
\end{tabular}

${ }^{\mathrm{a}}$ Pressures are 5 Torr; ${ }^{\mathrm{b}}$ Rate constants measured at 3 torr.

\subsubsection{Computational Method}

Reaction enthalpies for the abstraction and addition channels were calculated using the CBS-QB3 composite method, implemented within the Gaussian09 suite of programs. ${ }^{56}$ All geometries were first optimized using the B3LYP/CBSB7 method. All the vibrational frequencies are real, indicating that the optimized geometries represent minima on the potential energy surface.

Thus the reactions of hydroxyl radical with polycyclic aromatic hydrocarbon precursors were studied in a validated experimental set up mainly consisting of six-way cross stainless steel reaction chamber, pulsed (Nd:YAG and dye) laser systems, and detector (PMT) under vacuum of 1-7.5 Torr. Buffer gas and the gaseous reactants were injected using calibrated mass flow controllers. The photolysis laser beam used to generate radicals, probe laser beam used to monitor the concentration of hydroxyl radical, and the detector were orthogonal to each other. The enthalpy of the intermediates was computed using the CBS-QB3composite method.

\section{References:}

(1) Instruction Manual, iQ Dry pumping System; BOC Edwards.

(2) Collins, D.; Choosing Process Vacuum Pumps, Edwards Limited: 2012.

(3) Vacuum Pumps, https://people.rit.edu/vwlsps/LabTech/Pumps.pdf, 2008. 
(4) Fundamentals of Vacuum Technology; Umrath, W., Ed.; Oerlikon Leybold Vacuum, 2007.

(5) Dillon, T. J.; Tucceri, M. E.; Sander, R.; Crowley, J. N. Physical Chemistry Chemical Physics 2008, 10, 1540.

(6) Herbert, L. B.; Sims, I. R.; Smith, I. W. M.; Stewart, D. W. A.; Symonds, A.; Canosa, A.; Rowe, B. R. Journal of Physical Chemistry 1996, 100, 14928.

(7) Dejuan, J.; Smith, I. W. M.; Veyret, B. Journal of Physical Chemistry 1987, 91, 69.

(8) Dejuan, J.; Smith, I. W. M.; Veyret, B. Chemical Physics Letters 1986, 132, 108.

(9) Perry, R. A. Journal of Chemical Physics 1985, 82, 5485.

(10) Onel, L.; Blitz, M. A.; Breen, J.; Rickardcd, A. R.; Seakins, P. W. Physical Chemistry Chemical Physics 2015, 17, 25342.

(11) Onel, L.; Blitz, M. A.; Seakins, P. W. Journal of Physical Chemistry Letters 2012, 3, 853.

(12) Wollenhaupt, M.; Carl, S. A.; Horowitz, A.; Crowley, J. N. Journal of Physical Chemistry A 2000, 104, 2695.

(13) Dillon, T. J.; Crowley, J. N. Atmospheric Chemistry and Physics 2008, 8, 4877.

(14) Baasandorj, M.; Ravishankara, A. R.; Burkholder, J. B. Journal of Physical Chemistry A 2011, 115, 10539.

(15) Rajakumar, B.; Burkholder, J. B.; Portmann, R. W.; Ravishankara, A. R. Physical Chemistry Chemical Physics 2005, 7, 2498.

(16) Bocherel, P.; Herbert, L. B.; Rowe, B. R.; Sims, I. R.; Smith, I. W. M.; Travers, D. Journal of Physical Chemistry 1996, 100, 3063.

(17) Vakhtin, A. B.; Heard, D. E.; Smith, I. W. M.; Leone, S. R. Chemical Physics Letters 2001, 344, 317.

(18) Goulay, F.; Leone, S. R. Journal of Physical Chemistry A 2006, 110, 1875.

(19) Robertshaw, J. S.; Smith, I. W. M. Journal of Physical Chemistry 1982, 86, 785.

(20) Blitz, M. A.; Pesa, M.; Pilling, M. J.; Seakins, P. W. Journal of Physical Chemistry A 1999, 103, 5699.

(21) Rudich, Y.; Talukdar, R.; Burkholder, J. B.; Ravishankara, A. R. Journal of Physical Chemistry 1995, 99, 12188.

(22) Porter, G. Science 1968, 160, 1299.

(23) Smith, I. W. M. Berichte Der Bunsen-Gesellschaft-Physical Chemistry Chemical Physics 1982, 86, 395.

(24) Bach, R. D.; Ayala, P. Y.; Schlegel, H. B. Journal of the American Chemical Society 1996, 118, 12758.

(25) Amaral, G.; Xu, K. S.; Zhang, J. S. Journal of Physical Chemistry A 2001, 105, 1465.

(26) Reiche, F.; Abel, B.; Beck, R. D.; Rizzo, T. R. Journal of Chemical Physics 2000, 112, 8885.

(27) Brust, A. S.; Becker, K. H.; Kleffmann, J.; Wiesen, P. Atmospheric Environment 2000, 34, 13.

(28) Ning, C. L.; Pfab, J. Journal of Physical Chemistry A 1997, 101, 6008.

(29) Schiffman, A.; Nelson, D. D.; Nesbitt, D. J. Journal of Chemical Physics 1993, 98, 6935.

(30) Mishra, D. P. Experimental combustion : an introduction, 2014.

(31) German, K. R. Journal of Chemical Physics 1975, 63, 5252.

(32) German, K. R. Journal of Chemical Physics 1975, 62, 2584.

(33) Hogan, P.; Davis, D. D. Chemical Physics Letters 1974, 29, 555. 
(34) Goulay, F.; Rebrion-Rowe, C.; Le Garrec, J. L.; Le Picard, S. D.; Canosa, A.; Rowe, B. R. Journal of Chemical Physics 2005, 122.

(35) Goulay, F.; Rebrion-Rowe, C.; Biennier, L.; Le Picard, S. D.; Canosa, A.; Rowe, B. R. J. Phys. Chem. A 2006, 110, 3132.

(36) Trevitt, A. J.; Goulay, F.; Taatjes, C. A.; Osborn, D. L.; Leone, S. R. J. Phys. Chem. A 2010, 114, 1749.

(37) Sappey, A. D.; Crosley, D. R.; Copeland, R. A. Applied Physics B-Photophysics and Laser Chemistry 1990, 50, 463.

(38) Kiefer, J.; Li, Z. S.; Seeger, T.; Leipertz, A.; Alden, M. Proc. Comb. Inst. 2009, 32, 921.

(39) Klein-Douwel, R. J. H.; Luque, J.; Jeffries, J. B.; Smith, G. P.; Crosley, D. R. Appl. Opt. 2000, 39, 3712.

(40) Hollas, J. M. Modern spectroscopy; J. Wiley: Chichester; Hoboken, NJ, 2004.

(41) Nd:YAG Laser Specifications, Continuum, The High Energy Laser Company.

(42) Kazakevicius, A.; Peckus, D.; Boiko, O.; Valkunas, L.; Leonenko, E.; Telbiz, G.; Gulbinas, V. Journal of Physical Chemistry C 2015, 119, 19126.

(43) Wiedmann, J.; Penzkofer, A. Nuovo Cimento Della Societa Italiana Di Fisica B-General Physics Relativity Astronomy and Mathematical Physics and Methods 1981, 63, 459.

(44) Kubin, R. F.; Fletcher, A. N. Journal of Luminescence 1982, 27, 455.

(45) Zehentbauer, F. M.; Moretto, C.; Stephen, R.; Thevar, T.; Gilchrist, J. R.; Pokrajac, D.; Richard, K. L.; Kiefer, J. Spectrochimica Acta Part a-Molecular and Biomolecular Spectroscopy 2014, 121, 147.

(46) Dye Laser Specifications, Continuum, The High Energy Laser Company.

(47) Hamamatsu Photonics, Photomultiplier Tubes, Construction and Operating Characteristics, Connections to External Circuits; Photonics, H., Ed.; Hamamatsu Photonics.

(48) Skoog, D. A.; Holler, F. J.; Crouch, S. R. Principles of instrumental analysis, 2007.

(49) K.K., H. P. Photomultiplier Tubes-Basics and Applications; Third ed.; Hamamatsu Photonics K.K., 2007.

(50) Tully, F. P.; Goldsmith, J. E. M. Chemical Physics Letters 1985, 116, 345.

(51) Atkinson, R.; Pitts, J. N. Journal of Chemical Physics 1975, 63, 3591.

(52) Zhu, L.; Talukdar, R. K.; Burkholder, J. B.; Ravishankara, A. R. International Journal of Chemical Kinetics 2008, 40, 635.

(53) Davis, D. D.; Bollinger, W.; Fischer, S. Journal of Physical Chemistry 1975, 79, 293.

(54) Vakhtin, A. B.; Murphy, J. E.; Leone, S. R. Journal of Physical Chemistry A 2003, 107, 10055.

(55) Smith, R. H. Journal of Physical Chemistry 1983, 87, 1596.

(56) Frisch, M. J. T., G. W.; Schlegel, H. B.; Scuseria, G. E.; Robb, M. A.; Cheeseman, J. R.; Scalmani, G.; Barone, V.; Mennucci, B.; Petersson, G.A. et.al. Gaussian 092009 


\section{Chapter 3: Reaction of the OH Radical with Phenylacetylene}

\subsection{OH Radical Reaction with Aromatic Molecules}

The $\mathrm{OH}$ radical is one of the most abundant radical in combustion flames. Its reaction with benzene and substituted benzene molecules has been extensively studied both experimentally ${ }^{1-13}$ and theoretically. ${ }^{14,15}$ At room ${ }^{9,12}$ temperature and intermediate ${ }^{11}$ temperatures the dominant reaction entrance channel is predicted to be governed by electrophilic attack of the radical onto the aromatic ring to form a Van der Waals association complex. This pre-reactive complex isomerizes to a more stable addition intermediate which may decompose back to the reactants, be stabilized by collisional quenching, or decompose to give the final products. ${ }^{9}$ In the case of a long-lived addition intermediate the decomposition back to the reactants leads to biexponential decays of the $\mathrm{OH}$ radical concentration as observed for reactions with benzene, bromobenzene, toluene and its derivative., ${ }^{2,11}$ The presence of a substituent on the aromatic ring will affect the reaction rate by decreasing (electron donating or electron releasing or activating) or increasing (electron withdrawing or deactivating groups) the Gibbs free energy for the formation of the initial complex relatively to that of the OH-benzene complex. ${ }^{11}$ Witte et al. ${ }^{11}$ expressed this correlation using the Hammett equation ${ }^{16}$ that describes the electrophilic attack, specifically the addition to the aromatic ring. This equation relates the logarithm of the ratio of the rate coefficient for reaction of $\mathrm{OH}$ with a given molecule over that for benzene as a function of substituent constants as shown in equation $1 .^{11}$

$$
\log \left(\frac{\mathrm{k}}{\mathrm{k}_{0}}\right)=\sigma \rho
$$

where $\sigma$ is the substituent constant that relates the observed electronic (inductive or resonance) effect that a particular substituent imparts to the aromatic ring. Electron withdrawing substituents (e.g. $-\mathrm{NO}_{2}$ ) have positive $\sigma$ and electron donating substituents (e.g. $-\mathrm{NH}_{2}$ ) have negative $\sigma$. The 
constant $\rho$ measures the sensitivity of a particular reaction to changes in electronic effects of the substituent groups and depends on the nature of reactants and on the reaction conditions (e.g. temperature and solvent effect, etc.). Its sign and magnitude indicate charge build up during the progress of the chemical reaction. A value of $\rho>0$ is indicative of electron withdrawing groups that stabilizes the negative charge while a value $\rho<0$ is indicative of electron donating groups that stabilizes the positive charge. The magnitude of the reaction constant relates to the sensitivity of the reaction to the substituent effect. ${ }^{17}$

Assuming thermodynamic equilibrium between the reaction adduct and the reactants, the ratio of the reaction rate coefficients for the forward and backward reactions are related to the Gibb’s standard free energy by:

$\ln \left(\frac{\mathrm{k}_{\text {forward }}}{\mathrm{k}_{\text {backward }}}\right)=-\frac{\Delta \mathrm{G}^{0}}{\mathrm{RT}}$

By analogy with equation 1 we can state that the Hammett equation relates the association rate coefficients of two specific reactants to the Gibb's free energy of the aromatic-reactants association reaction. Figure 1 shows the semilog plot of the $\mathrm{OH}$ reaction rate constants with substituted aromatic rings against Hammett electrophilic substituent constants, showing a good linear correlation. Faster than expected reaction rates have been observed for molecules with deactivating or weakly activating substituents such as benzaldeheyde ${ }^{18}$ and styrene. ${ }^{12}$ In these cases hydrogen-abstraction or addition onto the substituent rather than addition onto the ring is likely to be the favorable entrance channel. This trend is helpful for the prediction of whether the addition of hydroxyl radical will occur onto the aromatic ring. 


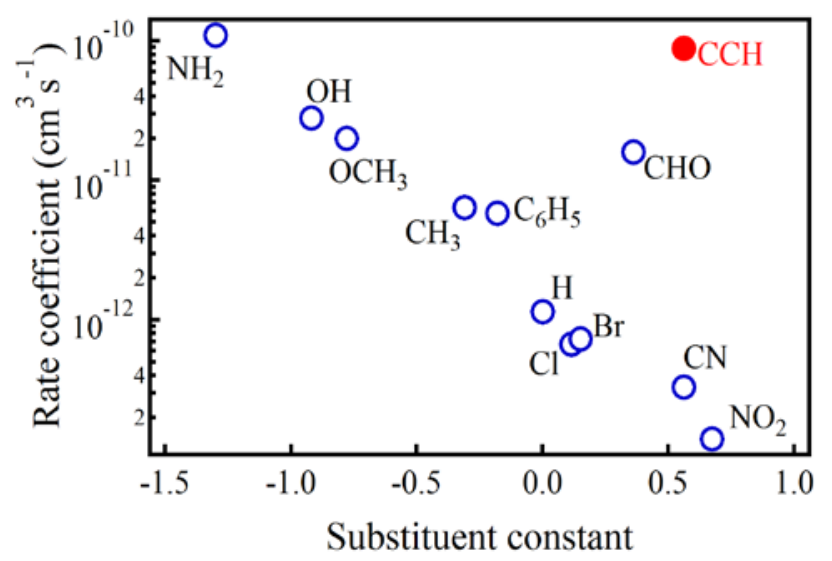

Figure 1. Plot of $\mathrm{OH}$ radical reaction rate coefficients against electrophilic substituent constants for monosubsituted aromatic compounds. ${ }^{11}$ The red dot is for phenylacetylene ${ }^{19}$

\subsection{Phenylacetylene in Combustion}

Phenylacetylene has been detected in the fuel rich premixed flames of monocyclic aromatic hydrocarbons ${ }^{20}$, the pyrolysis of cyclohexane ${ }^{21}$ and is believed to contribute to the formation of polycyclic aromatic hydrocarbon $(\mathrm{PAH})^{22}$. Spectroscopic evidence has suggested that phenylacetylene is one of the dissociative product of naphthalene. ${ }^{23}$ In addition, phenylacetylene along with naphthalene have been observed by photoionization mass spectrometry under combustion like conditions. ${ }^{24}$ As seen in Chapter 1, phenylacetylene is likely to be formed in combustion by the initial steps of the HACA mechanism, following the formation of benzene and/or the phenyl radical. Alternatively, benzene may react with the ethynyl radical $\left(\mathrm{C}_{2} \mathrm{H}\right)$ to form phenylacetylene through an addition/H-loss mechanism. ${ }^{25-27}$ The mostly likely reactions leading to phenylacetylene formation in combustion are summarized in scheme 1. 


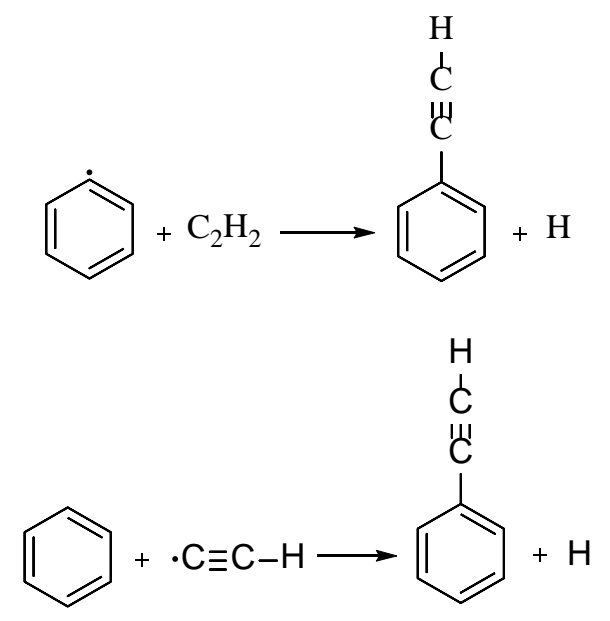

Scheme 1. Phenyl radical reacts with gaseous acetylene to form phenylacetylene or benzene reacts with ethynyl radical to form phenylacetylene

Once formed, consecutive reactions of phenylacetylene with atomic hydrogen and acetylene (scheme 2) may lead to the formation of the first PAH, naphthalene $\left(\mathrm{C}_{10} \mathrm{H}_{8}\right){ }^{28,29}$<smiles>C#Cc1ccccc1CCc1cccc2ccccc12</smiles>

Scheme 2. Phenylacetylene radical formed from phenylacetylene reacts with gaseous acetylene to form naphthalene

Despite its crucial importance in combustion, there is limited information about the $\mathrm{OH}+$ phenyl acetylene reaction ${ }^{30}$ and no kinetic information is available. Combustion models assume the reaction to mostly proceed through abstraction with a reaction rate slower than that of $\mathrm{OH}+$ benzene. ${ }^{29,31}$ Indeed, the deactivating effect of the ethynyl group $\left(-\mathrm{C}_{2} \mathrm{H}\right)$ is expected to decrease the rate of association with the aromatic ring. Electron delocalization effects and interaction of the radical with the $\pi$-electrons of the triple bonds are however, likely to lead to a rate that does not follow the empirical rule provided by Witte et al. ${ }^{11}$ The reaction rate coefficient of the $\mathrm{OH}+$ 
phenylacetylene reaction was measured over the 300-430 K temperature range in order to investigate the most likely reaction mechanism.

\subsection{Number Density of Phenylacetylene}

Phenylacetylene was purchased from Sigma Aldrich (98\%) and used without further purification. It is a yellow liquid at room temperature with 17.6 Torr vapor pressure at $37.7^{\circ} \mathrm{C}$. Approximately $5 \mathrm{~mL}$ of phenylacetylene was placed inside a bubbler contained in a regulated temperature bath (ISOTEMP 1016S). The bath was filled with water and cooled to $15{ }^{\circ} \mathrm{C}$ to prevent excess vaporization of phenylacetylene. It was completely degassed before use and the pressure was monitored using a baratron pressure gauge. A flow of $100 \mathrm{sccm}$ of helium was passed through the liquid to carry the phenylacetylene vapors. The fraction of phenylacetylene vapors in the flow was controlled using a needle valve placed just after the bubbler. Its vapor was introduced into a UV absorption cell used to measure the number density of phenylacetylene before mixing with buffer gas (helium) and $\mathrm{OH}$ radical precursor in a mixing cylinder.

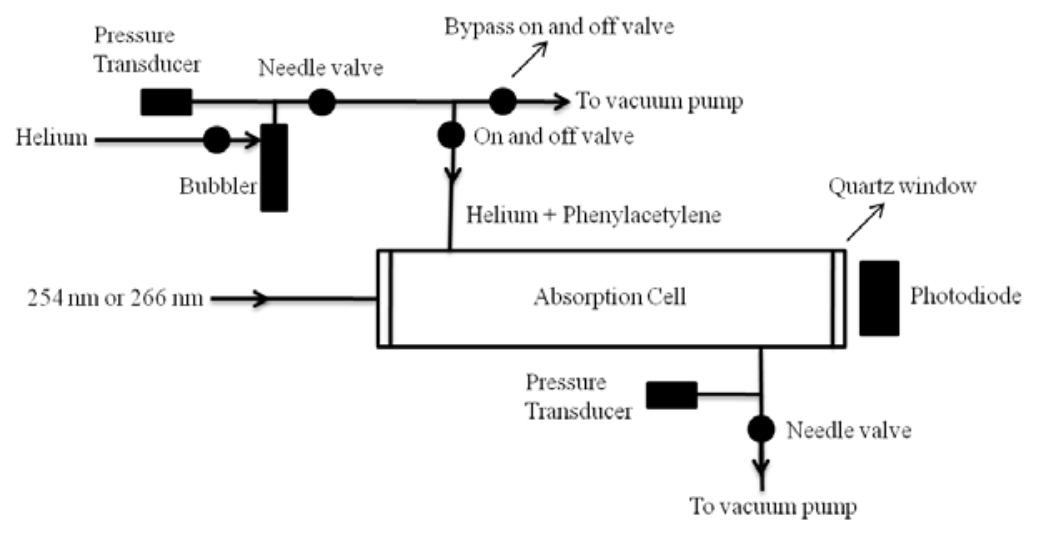

Figure 2. Schematic of experimental set up to determine the cross-section of phenylacetylene at $266 \mathrm{~nm}$

The number density of phenylacetylene was measured prior to its injection into the 
reaction chamber using UV-light absorption. Figure 2 displays a schematic of the experimental setup used for the light absorption measurements. The UV absorption cell is a $\sim 19 \mathrm{~cm}$ long stainless steel tube cylinder terminated by two 1" quartz windows, and maintained at room temperature. The transmitted intensities of a $254 \mathrm{~nm}$ mercury pen ray lamp after absorption by phenylacetylene were measured using a photo diode (Thorlabs-DET10A, Si Detector, 200-1100 $\mathrm{nm})$. The phenylacetylene absorption cross-section at $254 \mathrm{~nm}$ is $\sigma_{254 \mathrm{~nm}}=3.82 \times 10^{-18} \mathrm{~cm}^{2}{ }^{41}$ Capacitance manometers were used to measure the pressures in both the absorption cell and the bubbler. The number density of phenylacetylene in the absorption cell, $n_{a b s}$, were regulated by varying the total pressure in the bubbler at a constant bath temperature.

The transmitted lamp intensity was measured first without phenylacetylene and then again after the introduction of phenylacetylene. The number density of phenylacetylene were inferred from the value of the intensity ratios, absorption cross-section and path length using equation 3.

$\ln \frac{\mathrm{I}}{\mathrm{I}_{0}}=-\sigma \mathrm{n}_{\mathrm{abs}} \ell$

where $I_{0}$ is the initial intensity (zero absorption); I the intensity after absorption; $\sigma$ the crosssection of phenylacetylene and $\ell$ the length of the absorption cell, and $n_{a b s}$ is the number density in the absorption cell. With the number density in the absorption cell known, the number density of phenylacetylene in the reaction cell, $\mathrm{n}_{\text {pha }}$ was calculated using the relationship of equation 4 : $\mathrm{n}_{\text {pha }}=\mathrm{n}_{\mathrm{abs}} \frac{\mathrm{Q}_{\mathrm{abs}}}{\mathrm{Q}_{\text {Total }}} \cdot \frac{\mathrm{P}_{\text {reaction }}}{\mathrm{P}_{\mathrm{abs}}}$

where $Q_{a b s}$ is the flow rate through the bubbler/absorption cell, $Q_{t o t a l}$ is the total volumetric flow rate of gases in the reaction cell, $P_{\text {reaction }}$ and $P_{a b s}$ are pressure in the reaction vessel and absorption cell respectively. Figure 3 shows the number densities in the reaction cell as a 
function of bubbler pressure (Torr) together with a fit of the experimental data. The equation generated from the fit was used for all experimental cases to determine the number density of phenylacetylene in the experiment. Experiments were repeated at a given bubbler pressure multiple times resulting in a total of 24 data points, to evaluate measurement errors.

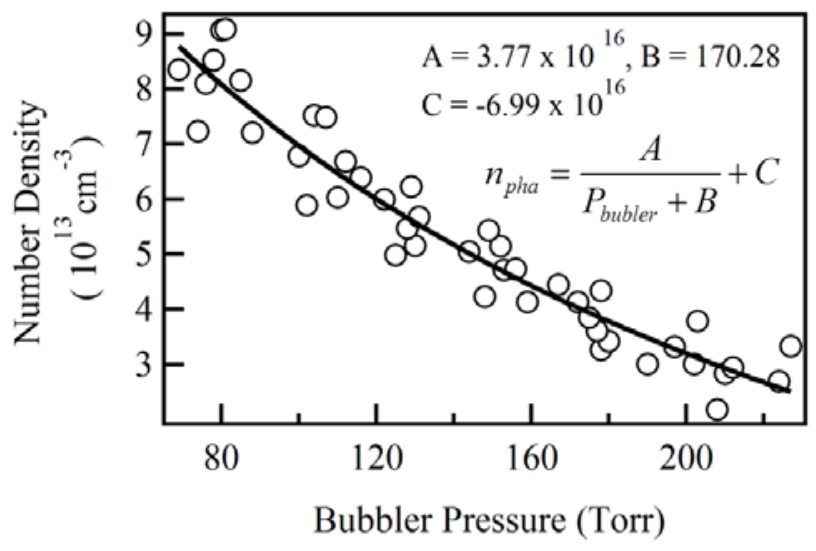

Figure 3. Bubbler pressure vs number density of phenyl acetylene in the reaction cell.

The solid line represents the fit relating the bubbler pressure to the number density of phenylacetylene, given by the equation. $\mathrm{A}, \mathrm{B}$ and $\mathrm{C}$ are constants from the fit and $\mathrm{P}_{\text {bubb }}$ is the bubbler pressure in Torr

\subsection{Determination of the Phenylacetylene Absorption Cross-Section at $266 \mathbf{~ n m}$}

Because the photolysis of the reactant by the pump laser may change its number density, it is important to obtain an order of magnitude of the phenylacetylene absorption cross section at $266 \mathrm{~nm}$. The experimental setup for the cross section measurements is identical to the one depicted in Figure 2. During the experiments, the pressure in the bubbler was increased using a needle valve to reach a pressure of around 500 Torr. At this time the bubbler was isolated from the inlet gas and absorption cell. The absorption cell was then evacuated in order to measure the initial intensity $\left(\mathrm{I}_{0}\right)$ using either the Mercury/Neon pen-ray lamp at $254 \mathrm{~nm}$ or the Nd:YAG laser beam at $266 \mathrm{~nm}$. The cell was then isolated from the vacuum pump and connected to the bubbler. 
After reaching a constant pressure, the absorption cell was isolated from the bubbler and the transmitted intensity was recorded. The pressure was then decreased using needle valve and four to six readings are recorded at each wavelength. The absorption cell was evacuated again to record the initial intensity.

The cross-section of phenylacetylene was determined using the Beer's law equation as shown in equation 5 .

$\ln \frac{\mathrm{I}}{\mathrm{I}_{0}}=-\sigma \mathrm{y} \frac{\mathrm{P}_{\mathrm{abscell}}}{\mathrm{kT}} \ell$

$I$ is the transmitted intensity, $I_{0}$ is the initial intensity, $\sigma$ is the cross-section of phenylacetylene, $y$ is the molar fraction of phenylacetylene in helium, $P_{\text {abscell }}$ is the pressure in the absorption cell, $k$ is the Boltzmann constant, $T$ is the absolute temperature in Kelvin, $\ell$ is the length of absorption cell. Figure 4 shows the typical plot of $\ln \frac{\mathrm{I}}{\mathrm{I}_{0}}$ vs. pressure in the absorption cell in Torr at 254 $\mathrm{nm}$. Using the above equation of Beer's law and the known value of the phenylacetylene crosssection of at $254 \mathrm{~nm}\left(3.82 \times 10^{-18} \mathrm{~cm}^{2}\right),{ }^{32}$ the molar fraction of phenylacetylene in helium was obtained from the slope of the data. 


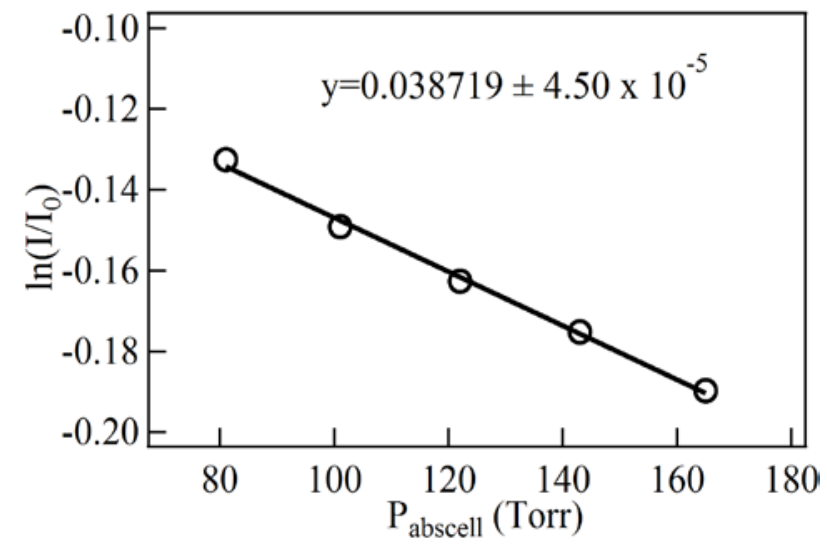

Figure 4. Typical plot of $\ln \frac{\mathrm{I}}{\mathrm{I}_{0}}$ vs Pabscell (Torr) at $254 \mathrm{~nm}$

Figure 5 shows a typical plot of $\ln \frac{\mathrm{I}}{\mathrm{I}_{0}} v s$. pressure in the absorption cell at $266 \mathrm{~nm}$. For known $y$ values, the phenylacetylene cross-section at $266 \mathrm{~nm}$ is inferred from the slope of the data.

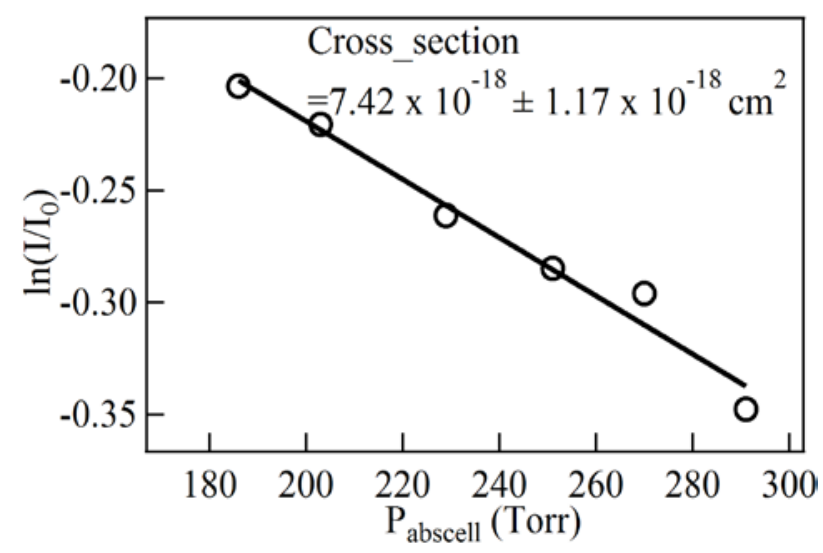

Figure 5. Typical plot of $\ln \frac{\mathrm{I}}{\mathrm{I}_{0}}$ vs Pabscell (Torr) at $266 \mathrm{~nm}$

The uncertainty on the phenylacetylene cross-section at $266 \mathrm{~nm}$ is calculated using the formula of equation 6 . 
$\Delta \sigma=\sqrt{\left(\frac{\Delta s}{s}\right)^{2}+\left(\frac{\Delta y}{y}\right)^{2}} . \sigma$

where $\Delta \sigma$ is the cross-section uncertainty, and $\Delta y$ the molar fraction uncertainty obtained from the plot at $254 \mathrm{~nm}, S$ is the slope obtained from fit to the data at $266 \mathrm{~nm}$ and $\Delta \mathrm{s}$ is its uncertainty. The uncertainty in the cross-section was calculated for each experiment and reported as two standard deviations. Table 1 shows all the cross-section values, $2 \sigma$ standard deviation and molar fraction of phenylacetylene in helium.

Table 1. Cross-section of phenylacetylene at $266 \mathrm{~nm}$, molar fraction of phenylacetylene in helium and standard deviation for each set of experiment

\begin{tabular}{|c|c|c|}
\hline $\begin{array}{c}\text { Molar fraction of } \\
\text { phenylacetylene }\end{array}$ & $\begin{array}{c}\text { Cross-section at } 266 \mathrm{~nm} \\
\left(\mathrm{~cm}^{2}\right)\end{array}$ & $\Delta \sigma\left(\mathbf{c m}^{2}\right)$ \\
\hline 0.025 & $1.42 \times 10^{-17}$ & $2.71 \times 10^{-18}$ \\
\hline 0.030 & $1.85 \times 10^{-17}$ & $1.40 \times 10^{-18}$ \\
\hline 0.049 & $1.03 \times 10^{-17}$ & $9.24 \times 10^{-19}$ \\
\hline 0.033 & $1.03 \times 10^{-17}$ & $2.66 \times 10^{-18}$ \\
\hline 0.021 & $1.08 \times 10^{-17}$ & $2.20 \times 10^{-18}$ \\
\hline 0.039 & $7.42 \times 10^{-18}$ & $1.17 \times 10^{-18}$ \\
\hline 0.034 & $1.42 \times 10^{-17}$ & $1.16 \times 10^{-18}$ \\
\hline 0.039 & $1.54 \times 10^{-17}$ & $2.62 \times 10^{-18}$ \\
\hline
\end{tabular}

Figure 6 shows all the cross-section values plotted against molar fraction of phenylacetylene in helium. The average of all the values is reported as the cross-section of phenylacetylene at $266 \mathrm{~nm}$ with $2 \sigma$ standard deviation and is $1.29 \times 10^{-17} \pm 7.11 \times 10^{-18} \mathrm{~cm}^{2}$. The red line is the average value of the cross-section of phenylacetylene at $266 \mathrm{~nm}$. The upper black line is the average cross-section plus $2 \sigma$ standard deviation and the lower black line is the average cross-section minus $2 \sigma$ standard deviation. 


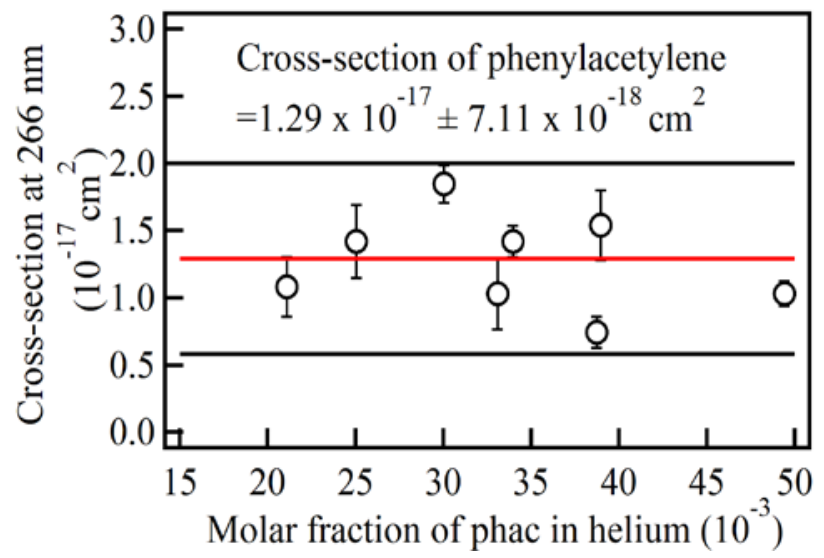

Figure 6. Plot of cross-section of phenylacetylene at $266 \mathrm{~nm}$ vs molar fraction of phenylacetylene in helium

\subsection{OH Radical Precursor}

\subsubsection{Hydrogen Peroxide}

For the initial kinetic experiments, hydrogen peroxide $\left(\mathrm{H}_{2} \mathrm{O}_{2}, 30 \%\right.$ by weight in $\left.\mathrm{H}_{2} \mathrm{O}\right)$ was purchased from Sigma Aldrich and used as the hydroxyl radical precursor. $\mathrm{H}_{2} \mathrm{O}_{2}$ was placed inside a bubbler at room temperature. Its vapor was carried using $100 \mathrm{sccm}$ of helium and mixed with phenylacetylene and the main helium buffer gas in the mixing cylinder before injection into the reaction chamber. $\mathrm{H}_{2} \mathrm{O}_{2}$ was photolysed at $266 \mathrm{~nm}$ using:

$\mathrm{H}_{2} \mathrm{O}_{2}+\mathrm{hv}(266 \mathrm{~nm}) \rightarrow 2 \mathrm{OH}$

\subsubsection{Nitrous Acid (HONO)}

Because of the high absorption cross-section of phenylacetylene at $266 \mathrm{~nm}$ an $\mathrm{OH}$ radical precursor at a longer wavelength had to be used. Nitrous acid readily photolyzes to $\mathrm{OH}$ and NO at $\lambda<390 \mathrm{~nm}^{33,34}$ HONO has an absorption maximum at $354.2 \mathrm{~nm}$, with an absorption cross- 
section of $2.98 \times 10^{-19} \mathrm{~cm}^{-233}$ The photolysis reaction is outlined below, with corresponding quantum yields $\varphi .^{35}$

$$
\begin{aligned}
\mathrm{HONO}+\mathrm{h} v(355 \mathrm{~nm}) & \rightarrow \mathrm{OH}+\mathrm{NO} \quad(\varphi=99 \%) \\
& \rightarrow \mathrm{H}+\mathrm{NO}_{2} \quad(\varphi=1 \%)
\end{aligned}
$$

At this wavelength, the relative $\mathrm{OH}$ concentration in the reaction chamber was estimated to be on the order of $\sim 10^{11} \mathrm{~cm}^{-3}$, based on the 355-nm absorption cross-section, laser fluence and concentration of HONO. HONO gas was prepared by reaction of $\mathrm{NaNO}_{2}$ salt with concentrated $\mathrm{H}_{2} \mathrm{SO}_{4}$ acid following the reaction $\mathrm{R} 2$ :

$2 \mathrm{NaNO}_{2}(\mathrm{aq})+\mathrm{H}_{2} \mathrm{SO}_{4}(\mathrm{aq}) \rightarrow 2 \mathrm{HONO}(\mathrm{g})+\mathrm{Na}_{2} \mathrm{SO}_{4}(\mathrm{aq})$

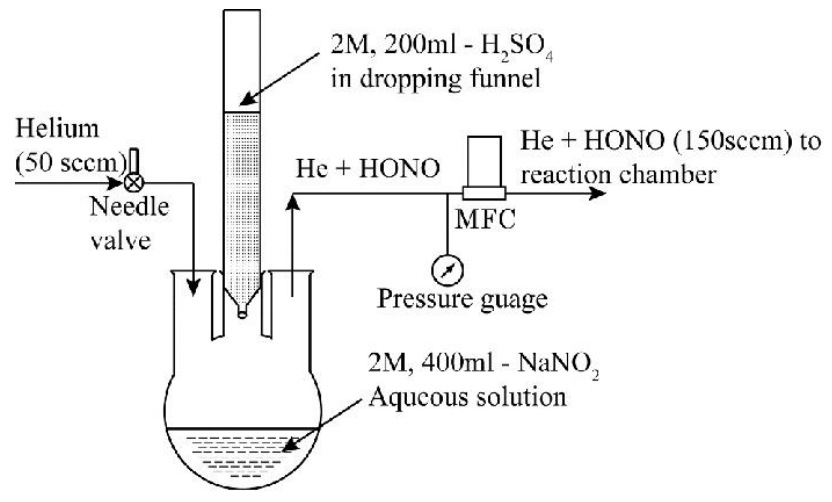

Figure 7. Schematic of experimental setup used for synthesis of HONO

The synthesis was based on a procedure outlined by Ning et. al. and Bagot et al. ${ }^{34,36}$ Figure 7 displays a schematic representation of the HONO synthesis set up. Aqueous solution of sodium nitrite $\left(\mathrm{NaNO}_{2}\right)(400 \mathrm{ml}, 2 \mathrm{M})$ was placed in a $1000 \mathrm{~mL}$-three necked flask, maintained at $-16^{\circ} \mathrm{C}$ using a methanol-ice bath Sulfuric acid $(2 \mathrm{M}, 200 \mathrm{ml})$ was held in a $250-\mathrm{mL}$ pressure equalizing dropping funnel connected to the three-necked flask via a standard ground glass joint. A capacitance manometer was used to monitor the pressure in the setup. The synthesis began 
with the evacuation of the setup to $<20$ Torr. After evacuation, $50 \mathrm{sccm}$ of helium was continuously passed through the setup via a needle valve and monitored using a mass flow controller. Reaction was initiated by adding sulfuric acid at a rate of 0.25 drops/second from the dropping funnel into the 3-neck flask. The reactants were stirred continuously using a magnetic stirrer. The products of the reaction were carried by the helium through a stainless steel lines resulting in a total regulated flow of $150 \mathrm{sccm}$. The reaction proceeded for approximately 2 hours with a constant output flowrate. The stability and constant HONO output was ascertained by monitoring the $\mathrm{OH}$ fluorescence signal over a 2-hour period, which was longer than the time required to obtain a full kinetic data set. $\mathrm{NO}_{2}$ gas may also form due to the dissociation of HONO, and be in equilibrium with HONO (R3).

$\mathrm{NO}_{2}+\mathrm{NO}+\mathrm{H}_{2} \mathrm{O} \leftrightarrow 2 \mathrm{HONO}$

Preparation of HONO by the salt acid method in other studies ${ }^{34-36}$ monitored the concentrations of $\mathrm{NO}_{2}$ and detected negligible amounts. To further ensure that no $\mathrm{NO}_{2}$ was formed, the entire synthesis was conducted at low pressures ( $<200$ Torr).

\subsection{Protection of the Laser Windows}

In order to minimize the deposition of photolytically decomposed reactants (phenylacetylene in our case) onto the windows, one of the four arms (photolysis beam entry arm) had a smaller cross-section ( $I D=0.4 ")$ with length of $8 "$. Deposition on the widows was avoided by countering the diffusion of phenylacetylene using a small flow (50 sccm) of helium adjacent to the window face. Figure 8 shows the modified long arm window used in the experiment of hydroxyl radical with phenylacetylene. It has $1 / 4$ " stainless steel tube for $\mathrm{q}_{\text {counterflow }}$ of helium to prevent the diffusion of phenylacetylene towards the quartz window. 


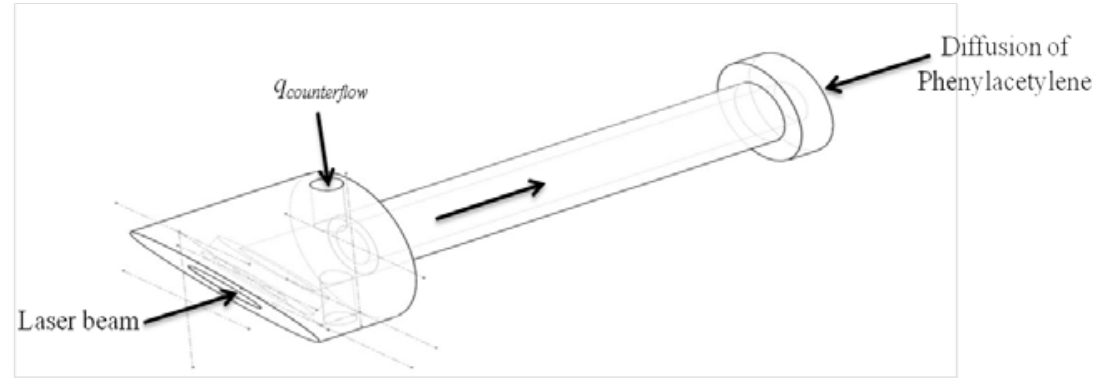

Figure 8. Long arm window to prevent the diffusion of phenylacetylene

The helium counterflow velocity was estimated using Fick's first law of diffusion. ${ }^{37}$ It relates the diffusion flux $(J)$, amount of substance per unit area per unit time, to the number density gradient $\mathrm{dn} / \mathrm{dx}$

$J=-D \frac{\mathrm{dn}}{\mathrm{dx}}$

where $\mathrm{D}$ is the diffusion coefficient (units $\mathrm{m}^{2} / \mathrm{s}$ )

By definition,

$\mathrm{J}=-\frac{\mathrm{n} \cdot \mathrm{dV}}{\mathrm{S} \cdot \mathrm{dt}}=-\frac{\mathrm{n} \cdot \mathrm{S} \cdot \mathrm{dl}}{\mathrm{S} \cdot \mathrm{dt}}=-\frac{\mathrm{n} \cdot \mathrm{S} \cdot \mathrm{v} \cdot \mathrm{dt}}{\mathrm{S} \cdot \mathrm{dt}}=-\mathrm{nv}$

$n$ is the number density, $S$ the surface area, $v$ the velocity, and $l$ the length.

We infer that

$\mathrm{D} \frac{\mathrm{dn}}{\mathrm{dx}}=\mathrm{nv}$

Over a characteristic length $l$, the equation can be approximated to:

$\mathrm{D} \frac{\mathrm{n}}{\mathrm{l}}=\mathrm{nv}$

resulting in

$\mathrm{V} \cong \frac{\mathrm{D}}{\mathrm{l}}$

The diffusion of phenylacetylene in He is unknown. Here it was approximated to that of a $\mathrm{He}-\mathrm{O}_{2}$ 
mixture, $0.697 \mathrm{~cm}^{2} / \mathrm{s}^{38,39}$ at $1 \mathrm{~atm}$. As the diffusion coefficient is inversely proportional to the pressure, the diffusion coefficient at 5 Torr is $0.0106 \mathrm{~m}^{2} \mathrm{~s}^{-1}$. For a characteristic length $l$ of the order of $1 \mathrm{~cm}$ the velocity is $1 \mathrm{~m} \mathrm{~s}^{-1}$. The corresponding flow rate in a tube of 0.4 " diameter is 30 sccm. All the experiments were performed with a He counter flow $50 \mathrm{sccm}$. With this flow, there was no significant deposition of phenylacetylene on the windows.

\subsection{Kinetic Measurements.}

\subsubsection{Pseudo-First Order Kinetics}

Within the pseudo first order approximation, corresponding to phenylacetylene (PAH precursor) being in large excess relative to the $\mathrm{OH}$ radical, the $\mathrm{OH}$ decay signal due to its reaction with phenylacetylene can be expressed using the following equations:

$$
\begin{aligned}
& {[\mathrm{OH}](t)=[\mathrm{OH}](t=0) e^{-k 1 s t t}} \\
& k_{1 s t}=k_{2 n d}[\text { phenylacetylene }]+k_{1 s t}^{\prime}
\end{aligned}
$$

where $k_{2 n d}$ represents the second order rate constant of the $\mathrm{OH}+$ phenylacetylene and $k_{1 s t}^{\prime}$ is the first order rate constant due to $\mathrm{OH}$ reaction with precursor molecules and other contaminants such as $\mathrm{NO}$ and $\mathrm{NO}_{2}$ produced during the photolysis and synthesis of $\mathrm{HONO}$, respectively. The integrated LIF signal is plotted against delay time for a known reactant number density and fitted using equation 12 to determine the corresponding $k_{1 s t}$.

Figure 9 shows typical decay traces plotted as a function of laser delay time for different phenylacetylene number densities. 


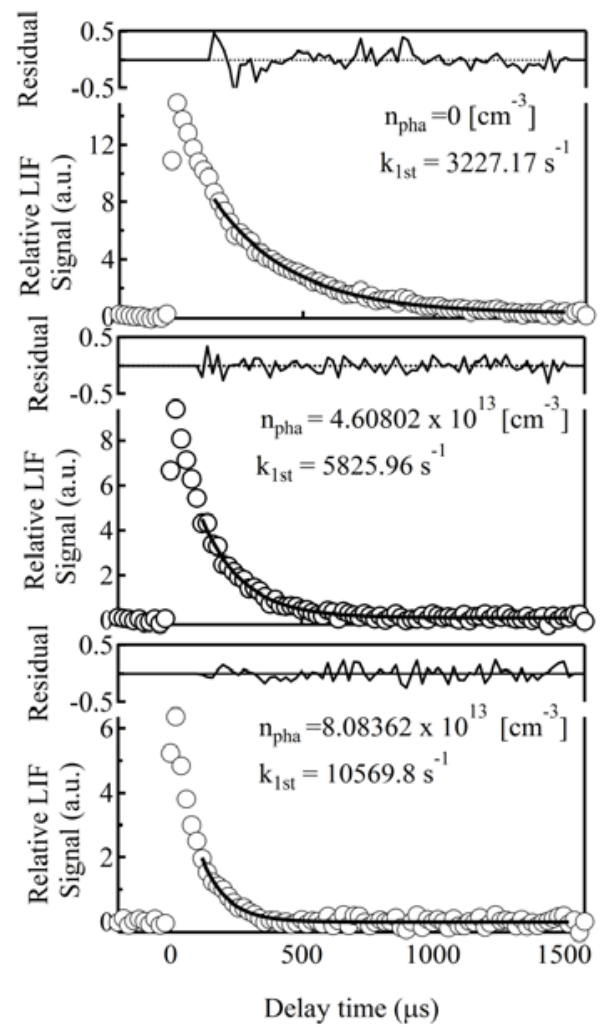

Figure 9. Typical traces of $\mathrm{OH}$ decays for varying concentrations of Phenyl acetylene at photolysis laser fluence of $147 \mathrm{~mJ} / \mathrm{cm}^{2}$. Plots are generated as a function of decay signal as a function of delay time between the probe laser and photolysis laser, fit to a single exponential function with corresponding residuals and $\mathrm{k}_{1 \mathrm{st}}$ values.

Figure 9 also includes exponential fits for three selected number densities of phenylacetylene and corresponding residuals at a photolysis laser power of $147 \mathrm{~mJ} \mathrm{~cm}^{-2}$. The data are fit $80 \mu \mathrm{s}$ after the pump laser pulse in order to avoid any effect from collisional relaxation of initially excited $\mathrm{OH}$ radicals. The experiments were repeated for different concentrations of phenylacetylene. Figure 10 shows a typical plot of $k_{1 s t}$ vs. concentration of reactant (phenylacetylene), for one data set. The slope of the linear fit is equal to the second order rate constant $\left(k_{2 n d}\right)$. 


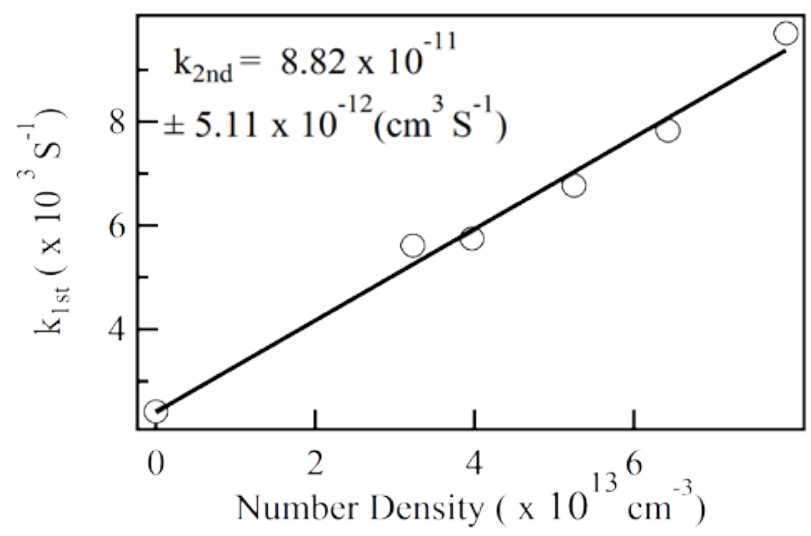

Figure 10. Typical traces of $\mathrm{OH}$ decays for varying concentrations of Phenyl acetylene at photolysis laser fluence of $147 \mathrm{~mJ} / \mathrm{cm}^{2}$. Plots are generated as a function of decay signal as a function of delay time between the probe laser and photolysis laser, fit to a single exponential function with corresponding residuals and $\mathrm{k}_{1 \mathrm{st}}$ values.

\subsubsection{Laser Power Dependence of Phenylacetylene}

Rate coefficients were measured at laser fluences ranging from 30 to $147 \mathrm{~mJ} \mathrm{~cm}^{-2}$. The laser power of the photolysis laser were tuned by changing the Q-Switch time delay in the pump (Nd:YAG) laser. By increasing the delay time ( $\mu$ s) the output power can be reduced. 


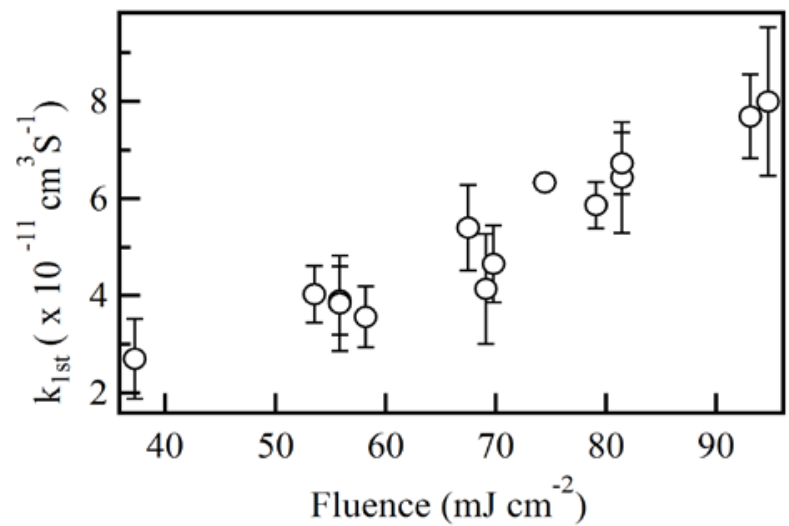

Figure 11. Power dependence of the second order rate constant $\left(\mathrm{k}_{2 \mathrm{nd}}\right)$ plotted as a function of fluence of photolysis laser at $266 \mathrm{~nm}$. The error bars for individual $\mathrm{k}_{2 n d}$ values are $2 \sigma$ precision.

Figure 11 shows results from 266-nm experiments demonstrating a positive fluence dependence of the second order rate constant $\left(k_{2 n d}\right)$. The high 266-nm absorption cross-section of phenyl acetylene possibly induces single and/or multiphoton phenomena and is likely to be responsible for the observed fluence dependence. Since the cross-section of phenyl acetylene at $266 \mathrm{~nm}$ is not available in the literature, it was measured and reported in section 3.4. The measured absorption cross-section of phenyl acetylene at $266 \mathrm{~nm}$ with $2 \sigma$ standard deviation is reported to be $1.29 \times 10^{-17} \pm 7.1 \times 10^{-18} \mathrm{~cm}^{2}$. For comparison the absorption cross section of benzene at $266 \mathrm{~nm}$ is $2.15 \times 10^{-20} \mathrm{~cm}^{2} \cdot{ }^{40}$ This large absorption cross-section of phenylacetylene is consistent with the deposition of photolytically decomposed products onto the laser windows within few minutes of experiment. This high absorption cross-section was also likely to be responsible for the observed power dependence of the second order rate constant $\left(k_{2 n d}\right)$ measured at $266 \mathrm{~nm}$. 


\subsubsection{HONO as OH Precursor}

The observed fluence dependence of the rate constant required an alternative source of $\mathrm{OH}$ radical at a wavelength greater than $266 \mathrm{~nm}$. HONO is an attractive option as its photolysis at $355 \mathrm{~nm}$ results in $\mathrm{OH}$ radicals and NO. The absorption cross-section of phenyl acetylene at $355 \mathrm{~nm}$ is negligible. All further kinetic experiments were performed using 355-nm photolysis of HONO (Nitrous acid) to produce the $\mathrm{OH}$ radicals.

Figure 12 displays the measured rate against laser fluence at $355 \mathrm{~nm}$. The error bars are a result of weighted averaging over the multiple experimental runs. At this wavelength and within the experimental error bars, there is no fluence dependence of the rate constant.

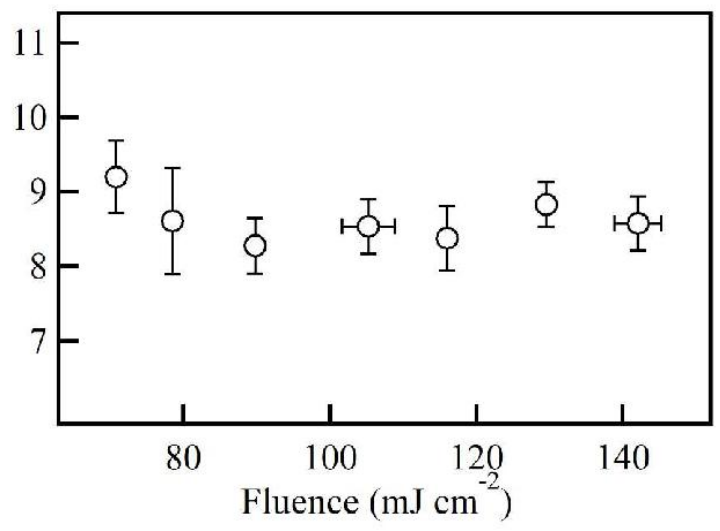

Figure 12. Plot of second order rate constant $\left(\mathrm{k}_{2} \mathrm{nd}\right)$ a function of laser fluence using HONO as radical source at $355 \mathrm{~nm}$.

Figure 13 shows the first order rate constant $\left(k_{1 s t}\right)$ plotted against phenyl acetylene number density. 


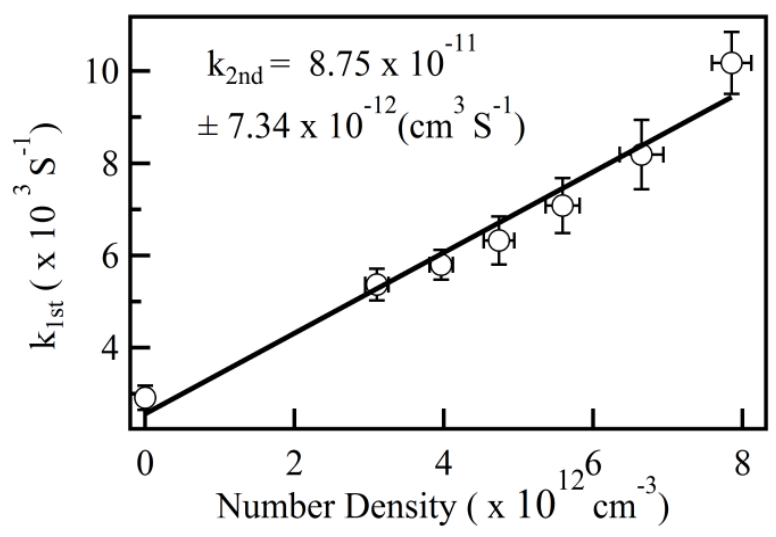

Figure 13. First order rate constants $\left(\mathrm{k}_{1 \mathrm{st}}\right)$ obtained from $\mathrm{OH}$ decay profiles plotted against concentrations of phenyl acetylene. The second order rate constant $\left(\mathrm{k}_{2 n d}\right)$ is the slope of the solid line obtained from a linear least-squares fit with $2 \sigma$ standard deviation.

The straight line obtained from a linear least-squares fit yields the second order rate constant $\left(k_{2 n d}\right)$. The horizontal error bars are due to differences in set bubbler pressures values as the experiments were conducted over numerous days. The vertical error bars for the first order rate constant are weighted averages of the respective measured $k_{1 s t}$ values obtained from single exponential fits with $2 \sigma$ precision similar to figure 9 .

\subsection{Pressure and Temperature Dependence of Phenylacetylene + OH Kinetics}

The pressure dependence of the $\mathrm{OH}+$ Phenyl acetylene reaction rate constants was studied over the 1-7.5 Torr pressure range. Figure 14 shows that the rate constants are pressure independent over the tested pressure ranges. Vertical error bars correspond to averaged values of $k_{2 n d}$ at a given pressure. 


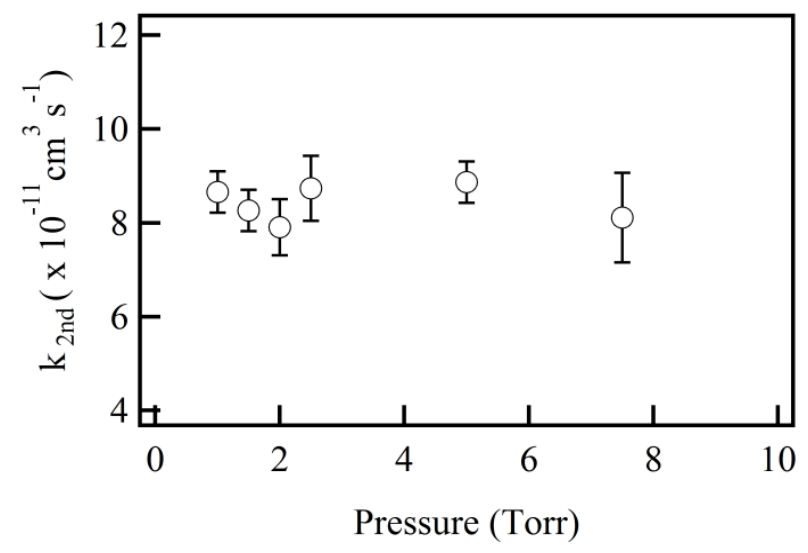

Figure 14. Plot of second order rate constant $\left(\mathrm{k}_{2 \mathrm{nd}}\right)$ of the reaction of $\mathrm{OH}$ from HONO photolysis at $355 \mathrm{~nm}$ with phenyl acetylene plotted against pressure of reaction cell. Each data point represents weighted averaged $k_{2 n d}$ value with $2 \sigma$ precision values obtained from multiple runs (minimum 5)

Figure 15 shows the temperature dependence of the reaction rates over the 293-423 K temperature range. Vertical error bars correspond to weighted averaged values of $k_{2 n d}$ for each temperature with $2 \sigma$ precision.

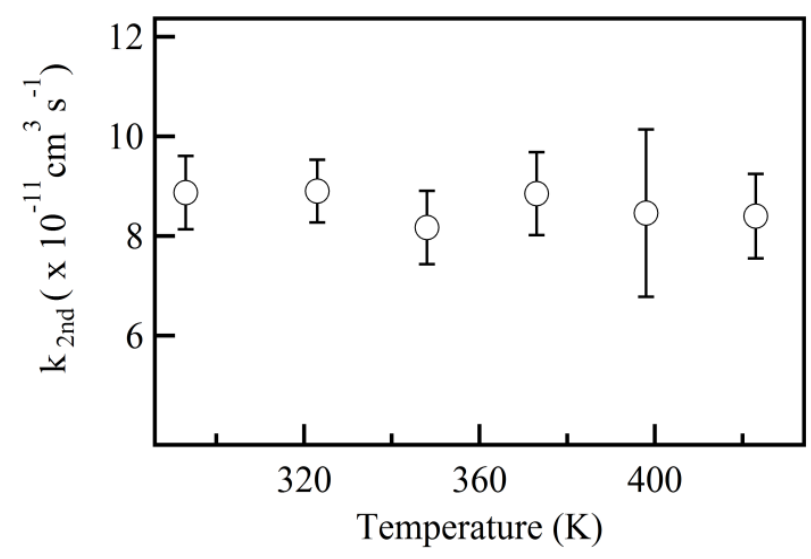

Figure 15. Second order rate constants $\left(\mathrm{k}_{2 n d}\right)$ for the reaction of $\mathrm{OH}$ from $\mathrm{HONO}$ photolysis at $355 \mathrm{~nm}$ with phenyl acetylene plotted against temperature of reaction cell (293 - 
$423 \mathrm{~K})$. Each data point represents weighted averaged $\mathrm{k}_{2 \mathrm{nd}}$ value with $2 \sigma$ precision values obtained from multiple runs (minimum 3, maximum 7)

\subsection{Discussion on the Reaction Mechanism}

Table 2 displays the reaction rates of the $\mathrm{OH}$ radical with a series of mono-substituted aromatic hydrocarbons. Strongly activating substituents such as $\mathrm{NH}_{2}-$ and $\mathrm{OH}-$ lead to reaction rates up to two orders of magnitude greater than that of $\mathrm{OH}+$ benzene while strongly deactivating substituents such as $\mathrm{NO}_{2}-$ and $\mathrm{CN}$ - slow down the reaction by a factor of ten. ${ }^{11}$ As discussed in the introduction, the reaction rates for $\mathrm{OH}+$ benzaldehyde and $\mathrm{OH}+$ styrene do not relate to the substituent effect likely due to the abstraction of the weakly bond aldehydic hydrogen and association with the carbon double bond, respectively. The $\mathrm{C}_{2} \mathrm{H}-$ group is isoelectronic to the $\mathrm{CN}$ - group and the electrophilic attack of the $\mathrm{OH}$ radical onto the aromatic ring of phenyl acetylene should therefore lead to a slower rate than that of the $\mathrm{OH}+$ benzene reaction. The fast reaction rate measured for phenyl acetylene compared to those for cyanobenzene ${ }^{11}$ and benzene ${ }^{11}$ suggests that the $\mathrm{C}_{2} \mathrm{H}$ - substituent plays an active role in the entrance channel. In the following paragraphs we discuss the entrance pathways that are consistent with a fast reaction rate. 
Table 2. Rate coefficients $\left(\mathrm{cm}^{3} \mathrm{~s}^{-1}\right)$ for the $\mathrm{OH}$ reaction with various mono-substituted aromatic rings

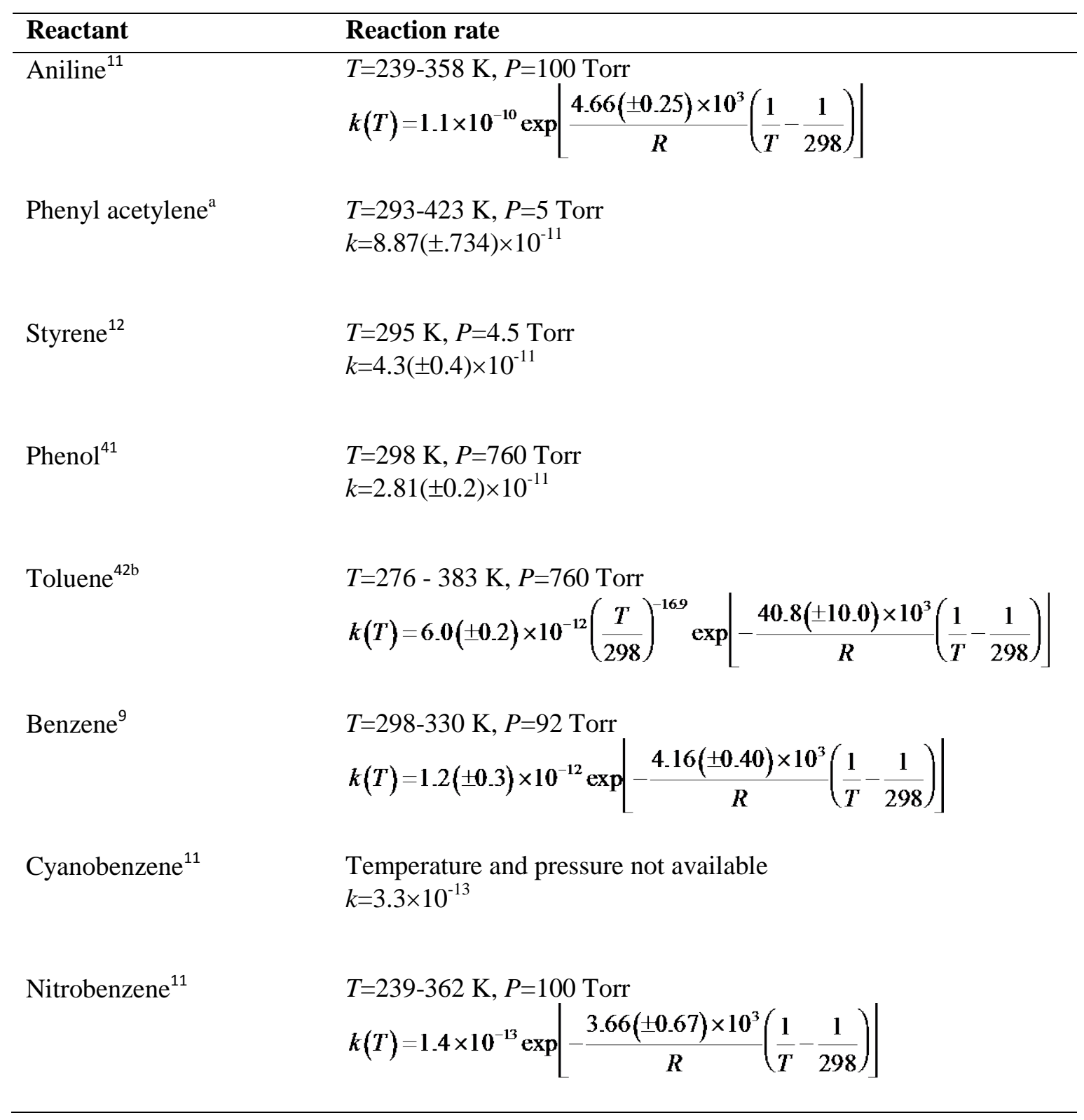

Table 3 displays the computed enthalpies for the abstraction and $\mathrm{H}$-loss products for the $\mathrm{OH}+$ phenyl acetylene reaction. The heat of formation of reactants, products, and reaction intermediates are calculated using the CBS-QB3 composite method implemented within the Gaussian $09^{43}$ suite of programs. All the ring abstraction channels are found to be exoenergetic 
while the abstraction of the acetyleneic hydrogen is endoenergetic by $70.60 \mathrm{~kJ} \cdot \mathrm{mol}^{-1}$. In the case of the $\mathrm{OH}$ reaction with benzene, the direct interaction of the radical singly occupied $p$-orbital with a C-H $\sigma$-bond of the ring leads to a transition state $19.94 \mathrm{~kJ} \mathrm{~mol}^{-1}$ above the energy of the reactants. ${ }^{14}$ The transition state for abstraction of a hydrogen atom from the aromatic ring of phenyl acetylene is likely to be comparable to that for abstraction from benzene. The fast and temperature independent measured reaction rate suggests that direct abstraction of a hydrogen atom from phenyl acetylene is likely to be a negligible reaction pathway over the experimental 293-423 K temperature range.

Table 3. Enthalpy of reaction for the abstraction and H-loss channels of $\mathrm{OH}+$ Phenylacetylene

\begin{tabular}{|c|c|c|c|}
\hline Products & $\begin{array}{c}\Delta_{r} H(298 \mathrm{~K}) \\
\left(\mathrm{kJ.mol}^{-1}\right)\end{array}$ & Products & $\begin{array}{c}\Delta_{r} \mathrm{H}(298 \mathrm{~K}) \\
\left(\mathrm{kJ} \cdot \mathrm{mol}^{-1}\right)\end{array}$ \\
\hline $\begin{array}{l}\text { o-ethynylphenyl } \\
+\mathrm{H}_{2} \mathrm{O}\end{array}$ & -9.56 & $\begin{array}{c}\text { m-ethynylphenol } \\
+\mathrm{H}\end{array}$ & +1.35 \\
\hline $\begin{array}{l}\text { m-ethynylphenyl } \\
+\mathrm{H}_{2} \mathrm{O}\end{array}$ & -14.89 & $\begin{array}{c}\text { p-ethynylphenol } \\
+\mathrm{H}\end{array}$ & +1.12 \\
\hline $\begin{array}{c}\text { p-ethynylphenyl } \\
+\mathrm{H}_{2} \mathrm{O}\end{array}$ & -13.39 & $\begin{array}{l}\text { Phenylethynol } \\
+\mathrm{H}\end{array}$ & +41.97 \\
\hline $\begin{array}{l}\text { Benzoethynyl } \\
+\mathrm{H}_{2} \mathrm{O}\end{array}$ & +70.60 & $\begin{array}{c}\text { Phenylketene } \\
+\mathrm{H}\end{array}$ & -83.42 \\
\hline $\begin{array}{c}\text { o-ethynylphenol } \\
+ \text { H }\end{array}$ & +4.98 & $\begin{array}{c}\text { Benzofurane } \\
+\mathrm{H}\end{array}$ & -133.64 \\
\hline
\end{tabular}

The association of the $\mathrm{OH}$ radical with the $\pi$-electrons of either the aromatic ring or the triple bond of the acetyl group is likely to proceed through the barrier-less formation of a Van der Waals complex as predicted for benzene ${ }^{14}$ and diacetylene. ${ }^{44}$ The association pre-reactive complex may either decompose back to the reactants and/or further isomerize through different transition states to give more stable addition intermediates. Figure 16 displays the structure and 
computed enthalpies of the nine possible $\mathrm{OH}$ addition intermediates together with some of their isomers. The isomerization of the pre-reactive complex to a $\mathrm{H}_{2} \mathrm{O}$-phenylacetylenyl radical association complex as observed for $\mathrm{OH}+$ benzene is unlikely to be accessible over the experimental temperature range. ${ }^{14}$ The good exponential fits displayed in Figure 9 suggest that

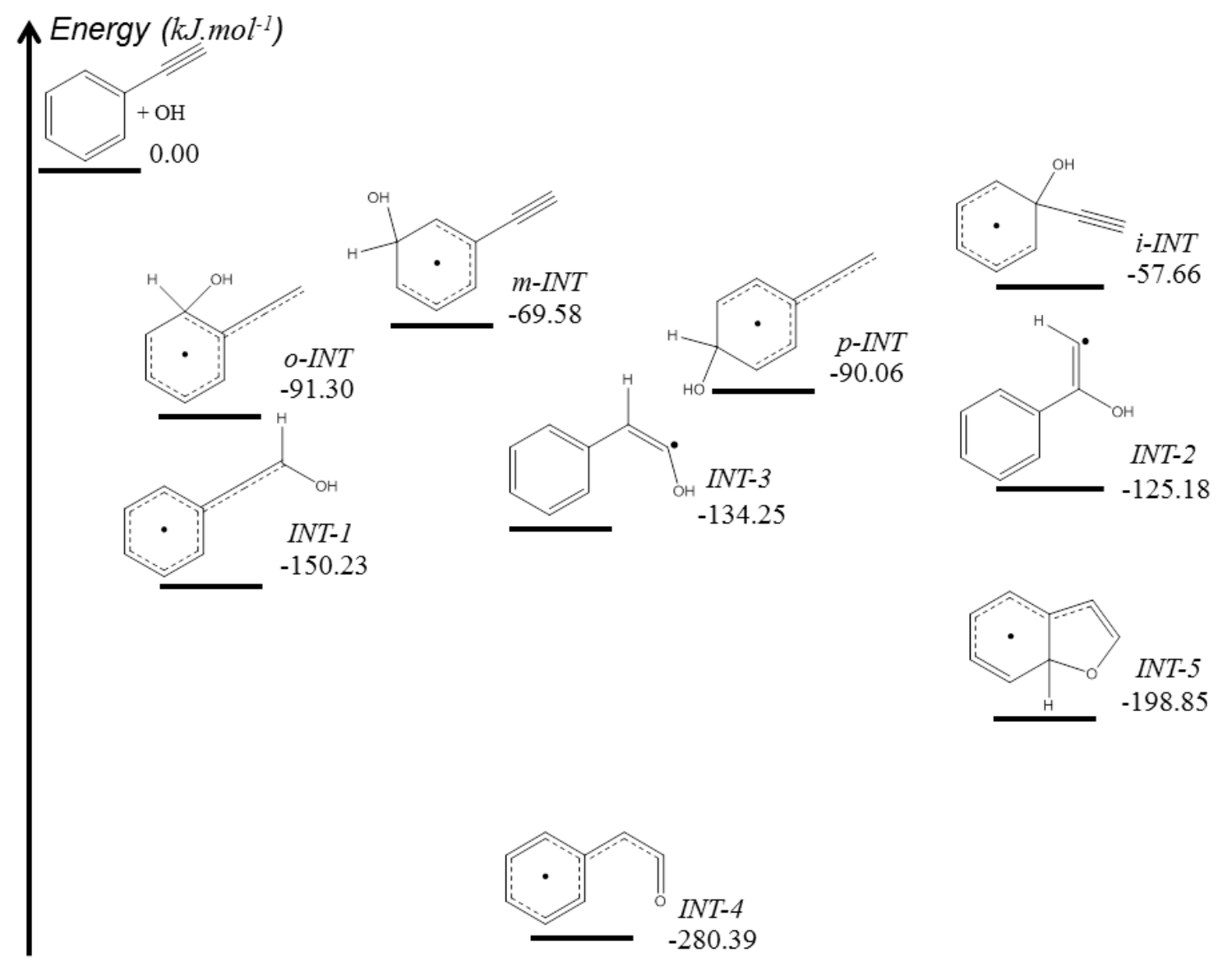

Figure 16. Exothermicites for the addition reaction intermediates and some of their isomers

the initial addition intermediate has a very short lifetime. This short-lived intermediate may still decompose back to the reactants without noticeable bi-exponential decays, decompose to final products, or be stabilized by collision with the buffer gas. In the latter case, an increase in total pressure will decrease the fraction of intermediates able to decompose back to the reactants and therefore increase the apparent reaction rate. Such behavior was observed for $\mathrm{OH}+$ benzene up 
to $40 \mathrm{Torr}^{9}$ and $\mathrm{OH}+$ styrene up to 2 Torr. $^{12}$ The lack of pressure dependence in Figure 9 suggests that the reaction already proceeds within the high-pressure limit at 1 Torr. Any initially formed association complex is therefore likely to isomerize to a more stable addition-like intermediate that is either stabilized by collisional quenching or decompose to the products without significantly decomposing back to the reactants. Although the kinetics data do not provide any further indications about its possible structure or fate, additional information may be inferred from the comparison with $\mathrm{OH}+$ benzen $^{14}$ and $\mathrm{OH}+$ diacetylene reactions. ${ }^{44}$

In Figure 16, the ortho (o-Add), meta (m-Add), para (p-Add), and ipso (i-Add) ringaddition intermediates may be formed by association with the aromatic ring while the Add- 1 and Add-2 intermediates may be formed by attack onto the triple bond. The direct addition onto the inner acetylenic carbon is likely to proceed through a transition state above the energy of the reactants as observed for the $\mathrm{OH}+$ diacetylene reaction. ${ }^{44}$ The resonantly stabilized INT-1 is therefore the most probable addition intermediate resulting from the radical association with the triple bond. The energy of the transition state corresponding to the conversion of the pre-reactive complex into INT-1 is likely to be decreased due to resonance and therefore to be below the transition state energy predicted for the terminal addition onto diacetylene (about $3 \mathrm{~kJ} \cdot \mathrm{mol}^{-1}$ ). ${ }^{44}$ In the case of benzene, the formation of addition intermediates from the initial association complex proceeds through a transition state $7 \mathrm{~kJ} \cdot \mathrm{mol}^{-1}$ above the energy of the reactants. ${ }^{14}$ Within the $2 \sigma$ standard deviations, the $\mathrm{OH}+$ phenyl acetylene reaction is likely to proceed without or with an activation barrier below $2 \mathrm{~kJ} \cdot \mathrm{mol}^{-1}$. Combined with the deactivating effect of the $\mathrm{C}_{2} \mathrm{H}-$ substituent, and over the experimental temperature and pressure ranges, the measured rates for the $\mathrm{OH}+$ phenyl acetylene reaction are consistent with the formation of the resonantly stabilized INT-1. The lack of pressure dependence suggests that collisional quenching with the buffer may 
stabilize a significant amount of INT-1. INT-1 has a mass of 119 , and this mass has been detected as a long-lived product in reaction of $\mathrm{OH}+$ phenyl acetylene using a quadrupole mass spectrometer. $^{30}$

\subsection{Conclusion}

The reaction of hydroxyl radical $(\mathrm{OH})$ with phenylacetylene was investigated using pulsed laser photolysis (PLP) and laser induced fluorescence (LIF). Absolute reaction rates were obtained over the 293 - $423 \mathrm{~K}$ temperature range and 1-7.5 Torr pressure range. The $\mathrm{OH}$ radicals were formed in the gas phase using either $\mathrm{H}_{2} \mathrm{O}_{2}$ at $266 \mathrm{~nm}$ or $\mathrm{HONO}$ at $355 \mathrm{~nm}$. The reaction rates measured at 355-nm photolysis wavelength were found to be independent of laser fluence and much faster than the $\mathrm{OH}+$ benzene reaction. The most likely reaction entrance channel and structure of the initially formed intermediates were discussed based on high-level calculations of the reaction intermediate energies as well as previous theoretical data on the $\mathrm{OH}+$ benzene $^{14}$ and $\mathrm{OH}+$ diacetylene ${ }^{44}$ reactions.

The rate of the $\mathrm{OH}+$ phenyl acetylene reaction was measured over a wide range of pressures, temperatures, and laser fluences. At 266-nm photolysis wavelength single photon and/or multiphoton effects were found to significantly affect the measured rate. This demonstrated the importance of ensuring the possibility of fluence dependence on the kinetic study of certain aromatic molecules. At 355-nm photolysis wavelength, within the experimental error bars, the rate was found to be independent of fluence, pressure and temperature. The average value was greater than expected based solely on the electrophilic attack of the radical onto the aromatic ring. Together with high-level calculations of the entrance pathways exothermicities, these results were consistent with the formation of an association pre-reactive complex involving the ethynyl group. The Van der Waals intermediate was then likely to evolve 
without or with a small activation energy to a resonantly stabilized addition intermediate, INT-1 (see Figure 16). The good exponential fits of the $\mathrm{OH}$ temporal decays and the lack of pressure dependence suggested that this intermediate did not decompose back to the reactants but was stabilized by collisional quenching.

The $\mathrm{OH}+$ phenylacetylene reaction rate was measured to be several order of magnitude greater than the values currently used in combustion models and might therefore play a significant role in phenylacetylene removal and formation of oxidation products. ${ }^{18}$ Under flame conditions (higher pressures and higher temperatures), other addition intermediates displayed in Figure 16 may become accessible. Abstraction of a hydrogen atom from the ring will also compete with the association/addition mechanism. Abstraction of the acetylenic hydrogen is likely to still be negligible. H-loss channels from the addition intermediates may become significant compared to collisional quenching of the intermediate. Table 3 displays the enthalpy of formation for several of the possible H-loss co-products. The most stable products are found to be phenylketene and benzofurane. All the ring substitution products are found to be above the energy of the reactants, although they will be accessible at high temperatures. Formation of phenylethynol is thermodynamically strongly disfavored. The formation of phenol from $\mathrm{OH}$ addition onto benzene is predicted to have a transition state $53.14 \mathrm{~kJ} \mathrm{~mol}^{-1}$ above the energy of the reactants while the substitution products and ketene-like products from $\mathrm{OH}+$ diacetylene are expected to proceed with submerge transition states. ${ }^{40}$ Products from the addition onto the acetyl group of phenylacetylene are therefore likely to be more favorable at high temperature that the substituted phenol molecules.

The formation of phenylketene would require the formation of INT-3 (see Figure 16) through a 1,2-hydrogen shift followed by H-loss of the hydrogen initially from the radical. The 
formation of benzofurane through H-loss would require a second H-shift from INT-3 to INT-4 followed by attack of the oxygen atom onto the ring to form the stable cyclic intermediate INT-5. Theoretical calculations on the $\mathrm{C}_{8} \mathrm{H}_{7} \mathrm{O}$ potential energy surface are necessary in order to predict if phenylketene and benzofurane will be significant reaction products at high temperatures. Nevertheless, our data set provide important information for combustion modeling and provides a detailed set of observables for future theoretical studies.

\section{References:}

(1) Aschmann, S. M.; Arey, J.; Atkinson, R. Journal of Physical Chemistry A 2013, 117, 2556.

(2) Bohn, B.; Zetzsch, C. Physical Chemistry Chemical Physics 2012, 14, 13933.

(3) Wang, L.; Arey, J.; Atkinson, R. Environmental Science \& Technology 2006, 40, 5465.

(4) Loison, J.-C.; Rayez, M.-T.; Rayez, J.-C.; Gratien, A.; Morajkar, P.; Fittschen, C.; Villenave, E. Journal of Physical Chemistry A 2012, 116, 12189.

(5) Colomb, A.; Jacob, V.; Kaluzny, R.; Baussand, R. International Journal of Chemical Kinetics 2004, 36, 367.

(6) Kramp, F.; Paulson, S. E. Journal of Physical Chemistry A 1998, 102, 2685.

(7) Thiault, G.; Mellouki, A.; Le Bras, G. Physical Chemistry Chemical Physics 2002, 4, 2194.

(8) Goumri, A.; Pauwels, J. F.; Devolder, P. Canadian Journal of Chemistry-Revue Canadienne De Chimie 1991, 69, 1057.

(9) Lorenz, K.; Zellner, R. Berichte Der Bunsen-Gesellschaft-Physical Chemistry Chemical Physics 1983, 87, 629.

(10) Berndt, T.; Boge, O. Physical Chemistry Chemical Physics 2006, 8, 1205.

(11) Witte, F.; Urbanik, E.; Zetzsch, C. Journal of Physical Chemistry 1986, 90, 3251.

(12) Baulch, D. L.; Campbell, I. M.; Saunders, S. M.; Louie, P. K. K. Journal of the Chemical Society-Faraday Transactions Ii 1989, 85, 1819.

(13) Taylor, S. E.; Goddard, A.; Blitz, M. A.; Cleary, P. A.; Heard, D. E. Physical Chemistry Chemical Physics 2008, 10, 422.

(14) Hollman, D. S.; Simmonett, A. C.; Schaefer, H. F. Physical Chemistry Chemical Physics 2011, 13, 2214.

(15) Chen, C. C.; Bozzelli, J. W.; Farrell, J. T. Journal of Physical Chemistry A 2004, 108, 4632.

(16) Brown, H. C.; Okamoto, Y. Journal of the American Chemical Society 1958, 80, 4979.

(17) March, J. Advanced organic chemistry : reactions, mechanisms, and structure; Wiley: New York, 1985.

(18) Niki, H.; Maker, P. D.; Savage, C. M.; Breitenbach, L. P. Journal of Physical Chemistry 1978, 82, 132.

(19) Kailasanathan, R. K. A.; Thapa, J.; Goulay, F. Journal of Physical Chemistry A 2014, $118,7732$. 
(20) Li, Y.; Zhang, L.; Yuan, T.; Zhang, K.; Yang, J.; Yang, B.; Qi, F.; Law, C. K. Combustion and Flame 2010, 157, 143.

(21) Wang, Z.; Cheng, Z.; Yuan, W.; Cai, J.; Zhang, L.; Zhang, F.; Qi, F.; Wang, J. Combustion and Flame 2012, 159, 2243.

(22) Richter, H.; Benish, T. G.; Mazyar, O. A.; Green, W. H.; Howard, J. B. Proceedings of the Combustion Institute 2000, 28, 2609.

(23) Bouwman, J.; de Haas, A. J.; Oomens, J. Chemical Communications 2016, 52, 2636.

(24) Parker, D. S. N.; Kaiser, R. I.; Troy, T. P.; Ahmed, M. Angewandte Chemie-International Edition 2014, 53, 7740.

(25) Goulay, F.; Leone, S. R. Journal of Physical Chemistry A 2006, 110, 1875.

(26) Woon, D. E. Chemical Physics 2006, 331, 67.

(27) Landera, A.; Mebel, A. M.; Kaiser, R. I. Chemical Physics Letters 2008, 459, 54.

(28) Anderson, H.; McEnally, C. S.; Pfefferle, L. D. Proceedings of the Combustion Institute 2000, 28, 2577.

(29) Wang, H.; Frenklach, M. Combustion and Flame 1997, 110, 173.

(30) Sloane, T. M.; Brudzynski, R. J. Journal of the American Chemical Society 1979, 101, 1495.

(31) Frenklach, M.; Warnatz, J. Combustion Science and Technology 1987, 51, 265.

(32) Talrose, V. Y., A.A.; Usov,A.A.; Goncharova, A.N.; Leskin, N.A.;Messineva, N.V. UV/VIS Spectra UV/VIS Spectra in NIST Chemistry WebBook, NIST Standard Reference Database Number 69.

(33) Brust, A. S.; Becker, K. H.; Kleffmann, J.; Wiesen, P. Atmospheric Environment 2000, 34, 13.

(34) Ning, C. L.; Pfab, J. Journal of Physical Chemistry A 1997, 101, 6008.

(35) Wollenhaupt, M.; Carl, S. A.; Horowitz, A.; Crowley, J. N. Journal of Physical Chemistry A 2000, 104, 2695.

(36) Bagot, P. A. J.; Waring, C.; Costen, M. L.; McKendrick, K. G. Journal of Physical Chemistry C 2008, 112, 10868.

(37) Paul, A. 2014.

(38) Marrero, T. R.; Mason, E. A. J. Phys. Chem. Ref. Data 1972, 1.

(39) Kestin, J., et.al. J .Phys.Chem. Ref. Data 1984, 13.

(40) Keller-Rudek, H.; Moortgat, G. K.; Sander, R.; Sörensen, R. Earth Syst. Sci. Data 2013, 5, 365.

(41) Rinke, M.; Zetzsch, C. Berichte Der Bunsen-Gesellschaft-Physical Chemistry Chemical Physics 1984, 88, 55.

(42) Anderson, P. N.; Hites, R. A. Environmental Science \& Technology 1996, 30, 1756.

(43) Frish, M. J. T., G.W.; Schlegel, H.B.; Scuseria, G.E.; Robb, M.A.; Cheeseman, J.R.; Scalmani, G.; Barone, V.; Mennucci, B.; Peterson, G.A.; et.al. Gaussian 092009.

(44) Senosiain, J. P.; Klippenstein, S. J.; Miller, J. A. Proceedings of the Combustion Institute 2007, 31, 185. 


\section{Chapter 4: Reaction of the OH Radical with Fulvenallene}

\subsection{Fulvenallene in Combustion Environments}

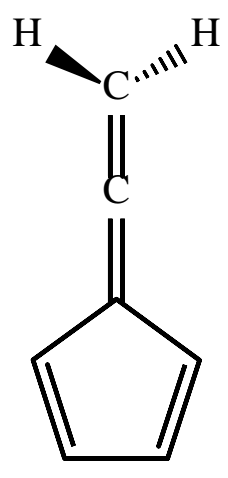

Figure 1. Fulvenallene

Fulvenallene (figure 1) is predicted to be an important intermediate in combustion environment. It is detected in cyclopentene $\left(\mathrm{C}_{5} \mathrm{H}_{10}\right)$ flame ${ }^{1}$ and is a primary unimolecular decomposition product of the benzyl radical $\left(\mathrm{C}_{7} \mathrm{H}_{7}\right)^{2-5}$ which in turn is a primary unimolecular decomposition product of toluene $\left(\mathrm{C}_{7} \mathrm{H}_{8}\right)^{4,6-16}$ and a photolysis product of benzyl radical. ${ }^{17,18} \mathrm{Da}$ Silva and Bozzelli ${ }^{19}$ investigated the hydrogen-abstraction mechanisms from fulvenallene by $\mathrm{H}-$ atoms, $\mathrm{OH}$, and $\mathrm{CH}_{3}$ radicals. In the case of the $\mathrm{OH}$ radical, the abstraction of a hydrogen atom from the $\mathrm{CH}_{2}$ group has a negative or no activation barrier, while the abstraction of a hydrogen atom from the carbon ring has an activation barrier of $9.6 \mathrm{~kJ} \mathrm{~mol}^{-1}$. This information, combined with the fact that the terminal $\mathrm{C}-\mathrm{H}$ bond of fulvenallene is $143.5 \mathrm{~kJ} \mathrm{~mol}^{-1}$ weaker than the ring $\mathrm{C}-\mathrm{H}$ bond, ${ }^{19}$ leads to fulvenallenyl being predicted as the most energetically and kinetically favorable abstraction product. The calculated room temperature abstraction rate coefficient $k_{A b s}$ is $1.45 \times 10^{-12} \mathrm{~cm}^{3} \mathrm{~s}^{-1}$ and is predicted to increase with temperature from 300 to $2000 \mathrm{~K}$ following the expression $k(T)=1.45 \times 10^{-12}\left(\frac{T}{298 K}\right)^{2.621} \exp \left(\frac{2.155 k J}{R}\left(\frac{1}{T}-\frac{1}{298 K}\right)\right) \mathrm{cm}^{3} \mathrm{~s}^{-1} \cdot{ }^{19}$ However, association of the $\mathrm{OH}$ radical with the $\pi$-electron system of unsaturated molecules is usually fast 
and likely to compete with the abstraction mechanism at low and intermediate temperatures. ${ }^{20-22}$ In the case of $\mathrm{OH}+$ allene $\left(\mathrm{CH}_{2} \mathrm{CCH}_{2}\right)$, at low temperatures the reaction proceeds through the formation of a van der Waals complex that can either isomerize into a reaction intermediate, or decompose back to the reactants. ${ }^{21,22}$ At temperatures above $600 \mathrm{~K}$, the reaction adduct formed by addition of the radical onto the allene is predicted to mainly dissociate back to $\mathrm{OH}+$ allene. $^{22}$ Overall, the $\mathrm{OH}+$ allene reaction rate coefficient is expected to decrease with temperature up to $600 \mathrm{~K}$, and to increase at higher temperatures, due to the increasingly favorable abstraction mechanism to form $\mathrm{C}_{3} \mathrm{H}_{3}+\mathrm{H}_{2} \mathrm{O}^{21,22}$ The presence of an allene and a cyclopentadiene group in fulvenallene suggests that the $\mathrm{OH}+$ fulevenallene reaction may proceed through similar competitive association/abstraction mechanisms as those predicted for the $\mathrm{OH}+$ allene reaction. For this reason the contribution of the $\mathrm{OH}+$ fulvenallene reaction to fulvenallenyl formation is likely to change with temperature and to be different in the interstellar medium or in combustion. This chapter presents the experimental investigation of the $\mathrm{OH}+$ fulvenallene reaction within the 298-450 K temperature range at 5 Torr starting with the synthesis and characterization of fulvenallene.

\subsection{Fulvenallene Synthesis and Characterization}

\subsubsection{Properties of Fulvenallene and its Precursor}

Table 1 shows the physical properties of the precursor homophthalic anhydride, intermediate product benzocyclobutenone and the main product fulvenallene. The physical properties of fulvenallene are unknown. Its vapor pressure is estimated to be approximately 30 Torr from the following experiment. Its melting and boiling points are still unknown. The vapor pressure of solid homophthalic anhydride at room temperature is estimated to be less than 1 Torr from the experiment. The vapor pressure of benzocyclobutenone is also unknown. 
Table 1. Physical properties of the reactant and products

\begin{tabular}{lccc}
\hline & $\begin{array}{c}\text { Homophthalic } \\
\text { anhydride }\end{array}$ & Benzocyclobutenone & Fulvenallene \\
\hline Molecular weight $\left(\mathbf{g ~ m o l}^{-\mathbf{1}}\right)$ & 162.14 & 118.13 & 90.12 \\
Melting point $\left({ }^{\circ} \mathbf{C}\right)$ & $140-142$ & $14^{23}$ & No data available \\
Boiling point $\left({ }^{\circ} \mathbf{C}\right)$ & No data available & $71-72(2 \text { Torr })^{23}$ & No data available \\
$\begin{array}{l}\text { Vapor pressure at room } \\
\text { temperature (Torr) }\end{array}$ & $<1^{\mathrm{a}}$ & No data available & $\sim 30^{\mathrm{a}}$ \\
\hline
\end{tabular}

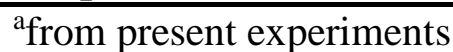

\subsubsection{Fulvenallene Synthesis}

Fulvenallene was synthesized by flash vacuum pyrolysis of homophthalic anhydride $\left(\mathrm{C}_{9} \mathrm{H}_{6} \mathrm{O}_{3}\right)$ as reported by Spangler et.al. ${ }^{24,25}$, likely following a mechanism proposed by Sakaizumi et al. ${ }^{26}$ in scheme 1.

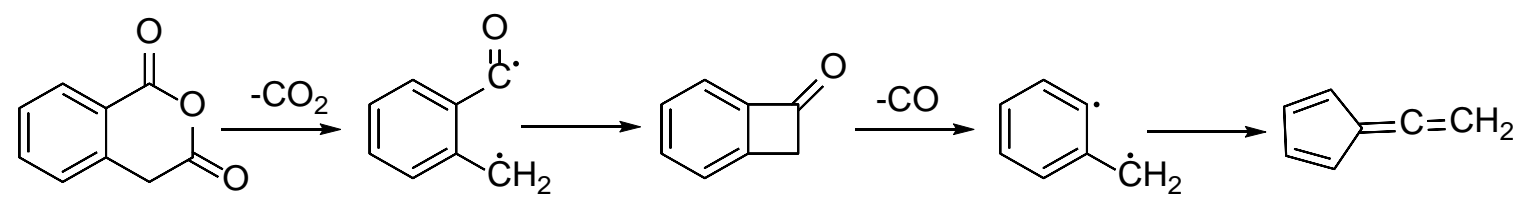

Homophthalic Anhydride Benzocyclobutenone Fulvenallene

Scheme 1. Proposed mechanism of fulvenallene formation

Figure 2 shows the experimental set up used to synthesize fulvenallene. The synthesis was performed in a 3-neck Pyrex flask containing a coiled 22-gauge 24-inch-long nichrome wire. The flask was connected to a 30-cm long water-cooled condensing column. All the ground glass joints and Teflon corks were sealed using high temperature grease. The outlet of the condenser was connected to a glass bubbler through $20 \mathrm{~cm}$ of 1/4"-PTFE tubing. About 2 to $3 \mathrm{~g}$ of homophthalic anhydride were mixed with sand and placed in the reaction flask. The flask, condensing column, and bubbler were pumped out by a rotary-valve pump for at least 2 hours 
(final pressure $<0.1$ Torr). The set-up was checked for leaks, after which the glass bubbler was submerged in liquid nitrogen.

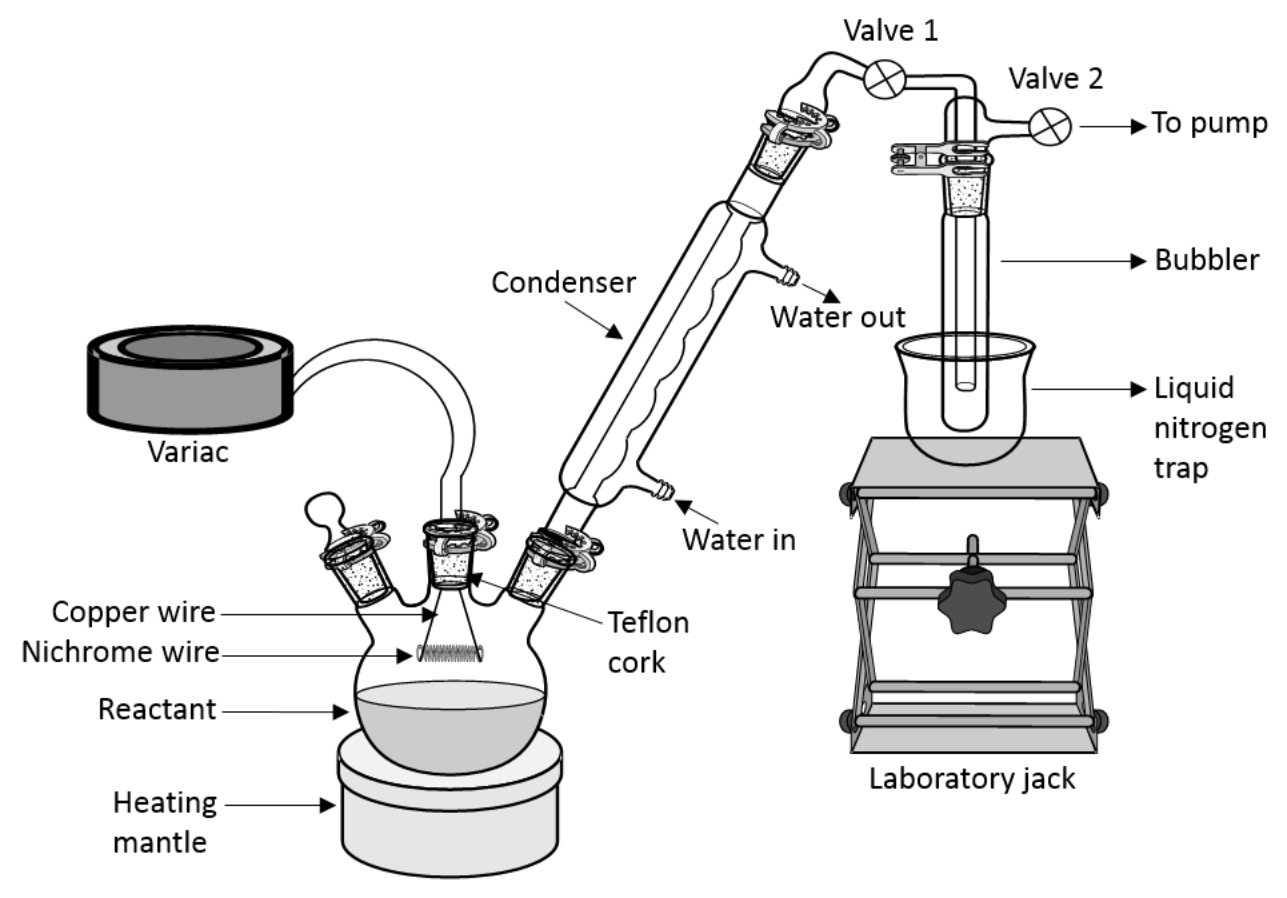

Figure 2. Experimental set up for the synthesis of Fulvenallene

The set-up was then isolated from the pump, and the reactor temperature was increased using a heating mantle, and subsequently monitored using a type- $\mathrm{K}$ thermocouple placed between the heating mantle and the flask. When the measured temperature exceeded approximately $110^{\circ} \mathrm{C}$, condensation of the starting material was observed on the uppermost sections of the flask, which was not being heated by the heating mantle. To reduce condensation, the upper part of the three-necked flask was isolated with heating tape. The condenser temperature was maintained at $75^{\circ} \mathrm{C}$ in order to avoid transfer of the reactant to the cold trap. Keeping the mantle temperature between $100^{\circ} \mathrm{C}$ and $110^{\circ} \mathrm{C}$ was found minimizing contamination by the homophthalic anhydride reactant in the final sample. 
When sufficient sublimation of the starting material was observed, the nichrome wire was resistively heated using a variable AC controller, resulting in a red-hot glow. This heating of the wire resulted in a fast increase of the temperature and pressure in the flask. To avoid overheating of the reactant, the heating mantle temperature was lowered to maintain a constant temperature between 110 and $155^{\circ} \mathrm{C}$. The bubbler trap was kept in liquid nitrogen $\left(-196^{\circ} \mathrm{C}\right)$ throughout the process. After 5 minutes of reaction, yellow product was observed on the inside wall of the inner bubbler tube, and the initial sand-reactant mixture inside the flask turned yellow, and eventually dark brown. The reaction was run for 30 to 60 minutes. The final pressure in each experiment varied from 45 Torr to 75 Torr. The pressure rise was likely to come from gas formation and possible leaking of the experimental setup at high temperatures.

After completion, the bubbler was isolated, and the liquid nitrogen bath was replaced by an acetone-liquid nitrogen slush $\left(-94^{\circ} \mathrm{C}\right)$. The bubbler was connected to the vacuum pump and evacuated to a pressure below 1 Torr. At this temperature, fulvenallene was expected to have a negligible vapor pressure while $\mathrm{CO}_{2}$ and $\mathrm{CO}$ were solid with high vapor pressures. Finally the acetone-liquid nitrogen slush was replaced by an ice-water bath to trap the reactant and benzocyclobutenone impurities. The solid yellow product turned into a liquid, with a typical increase of the pressure to $\sim 30$ Torr.

The bubbler was then connected to two room temperature 1-L stainless steel cylinders, initially under vacuum. After equilibration, the total pressure in the bubbler and stainless steel cylinders reached 10 to 15 Torr depending on how much product was formed. The cylinders were isolated from the glass bubbler, and pressurized with helium to reach a total pressure of 1000 Torr. The cylinders were then isolated, and used for IR characterization of the gas mixture or for the kinetics experiments. 


\subsubsection{Liquid Phase NMR Spectroscopy}

After synthesis, a fraction of the liquid product in the bubbler was extracted, dissolved in $\mathrm{CDCl}_{3}$, and analyzed by NMR spectroscopy. Formation of fulvenallene from phthalide or homophthalic anhydride thermal cracking was confirmed by comparing the experimental spectra to predicted ones. All the NMR spectra are in the appendix. As benzene was a co-product of phthalide cracking, and as it was not separable from fulvenallene, homophthalic anhydride was used as the reactant for all the experiments. The NMR spectra of the fulvenallene product were identical for both precursors. The ${ }^{1} \mathrm{H}$ NMR spectrum showed the presence of fulvenallene, benzocyclobutenone and homophthalic anhydride NMR analysis of liquid after collection and recondensation of gas mixture from the stainless steel cylinder showed mostly fulvenallene with traces of the reactants. Benzocyclobutenone was not observed after condensation of the cylinder mixture.

Even though fulvenallene was synthesized in the 1970s, its NMR data was neither documented nor found in the literature. For the $1^{\text {st }}$ time the ${ }^{1} \mathrm{H}$ and ${ }^{13} \mathrm{C}$ NMR spectra were obtained using a $600 \mathrm{MHz}$ INOVA NMR machine and its structure was confirmed by calculations (DT/GIAO and iterative simulation). ${ }^{1} \mathrm{D}$ and ${ }^{2} \mathrm{D} \mathrm{NMR},{ }^{1} \mathrm{H} /{ }^{13} \mathrm{C}$, selective-decoupled ${ }^{1} \mathrm{H}$ NMR spectrum, and proton coupled carbon NMR, NOESY, HSQC (heteronuclear single quantum coherence) and HMBC (heteronuclear multiband coherence) experiments are performed to assign all ${ }^{1} \mathrm{H}$ and ${ }^{13} \mathrm{C}$ NMR chemical shifts.

Spin-restricted density functional theory calculations were performed with the Gaussian 09 (G09) program. ${ }^{27}$ Geometry optimization and normal mode analysis was performed using the B3LYP functional and the $6-31+G(d)$ basis set to identify the ground state geometry of 
fulvenallene. ${ }^{28,29}$ The identified stationary point met or exceeded all default G09 convergence criteria. Using the same functional and the larger 6-311+G(d,p) and 6-311+G(3df,2p) basis sets, NMR chemical shift and spin-spin coupling constant calculations were performed using the GIAO method within G09. Isotropic chemical shifts were referenced relative to that of tetramethylsilane. The total energy, lowest frequencies, and the optimized coordinates for fulvenallene using 6-31+G(d,p) basis sets are given in the appendix.

Figure 3 shows the back to back representations of the calculated and experimental multiplicity patterns for $\mathrm{H} 1, \mathrm{H} 5 / \mathrm{H} 6$ and $\mathrm{H} 4 / \mathrm{H} 7$ protons of fulvenallene dissolved in $\mathrm{CDCl}_{3}$. The calculated coupling constants matched well with the experimental ones (see table 1 in annex).

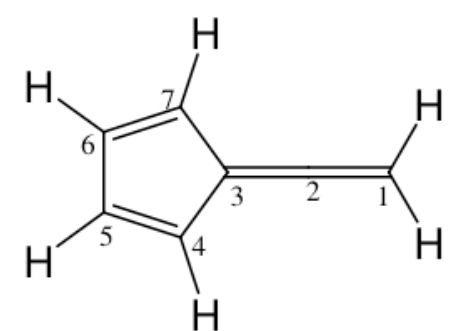

$\mathrm{H} 4 / \mathrm{H} 7$

(a)

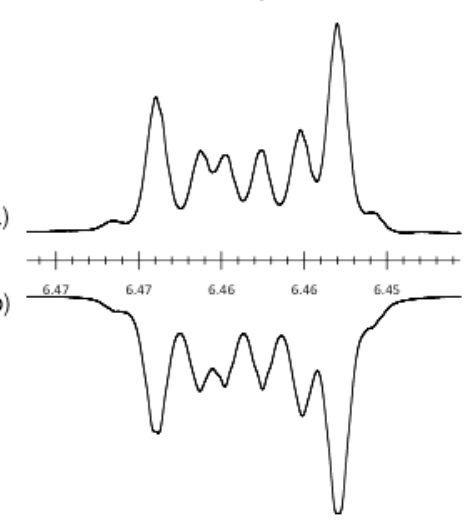

$\mathrm{H} 5 / \mathrm{H} 6$

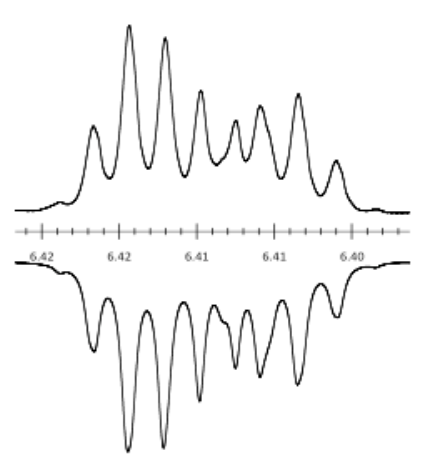

$\mathrm{H} 1$

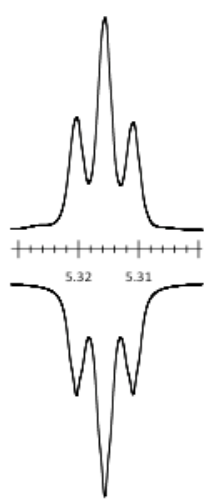

Figure 3. (a) Experimental and (b) simulated ${ }^{1} \mathrm{H}$ NMR spectrum of the liquid fulvenallene dissolved in $\mathrm{CDCl}_{3}$ 
The upfield signal at 5.32 belongs to C1 protons. It shows three peaks due to the spinspin interactions between 1- $\mathrm{CH}_{2}$ and $\mathrm{H} 4 / \mathrm{H} 7$ protons. The number of expected peaks or lines is determined by the following equation 1.

$\mathrm{N}=2 \mathrm{nI}+1$

Where $\mathrm{n}$ is the number of neighboring $\mathrm{H}$ nuclei and $\mathrm{I}$ is the spin quantum number $=1 / 2$

For $\mathrm{H} 1$ (C1) protons, there are 2 neighboring nuclei (H4/H7). So $\mathrm{N}=3$ peaks as shown in the figure 3 .

H5/H6 and H4/H7 protons exhibit complex splitting pattern. In other words the splitting patterns are not first order. This means the coupling constant values cannot be calculated for these protons. In order to determine coupling constants for these protons, simulation procedure (g-NMR software) are used that give all J values between protons of all three spin systems (H1, H4/H7, and H5/H6). See annex for the NMR spectra.

\subsubsection{Gas Phase FTIR Spectroscopy}

The composition of the gas mixture was determined using a $25-\mathrm{cm}^{3}$ double-pass gas cell (Mettler Toledo), coupled to a FTIR spectrometer (Mettler Toledo, ReactIR ic 15). Preliminary to the measurements, the gas cell was evacuated to a pressure below 1 Torr, and all the mirrors were flushed with dry nitrogen. A background spectrum was recorded by accumulating 1024 interferograms. The gas cell was then connected to the stainless steel cylinder containing the fulvenallene/He mixture. The total pressure in the absorption cell was set to 800 Torr. Spectra were recorded by accumulating 1024 interferograms with a resolution of $4 \mathrm{~cm}^{-1}$ or $8 \mathrm{~cm}^{-1}$. Figure 4 displays the average of 12 spectra recorded with a resolution of $4 \mathrm{~cm}^{-1}$. The spectra are baseline subtracted by fitting a $5^{\text {th }}$-order polynomial through the region displaying no significant signal. 

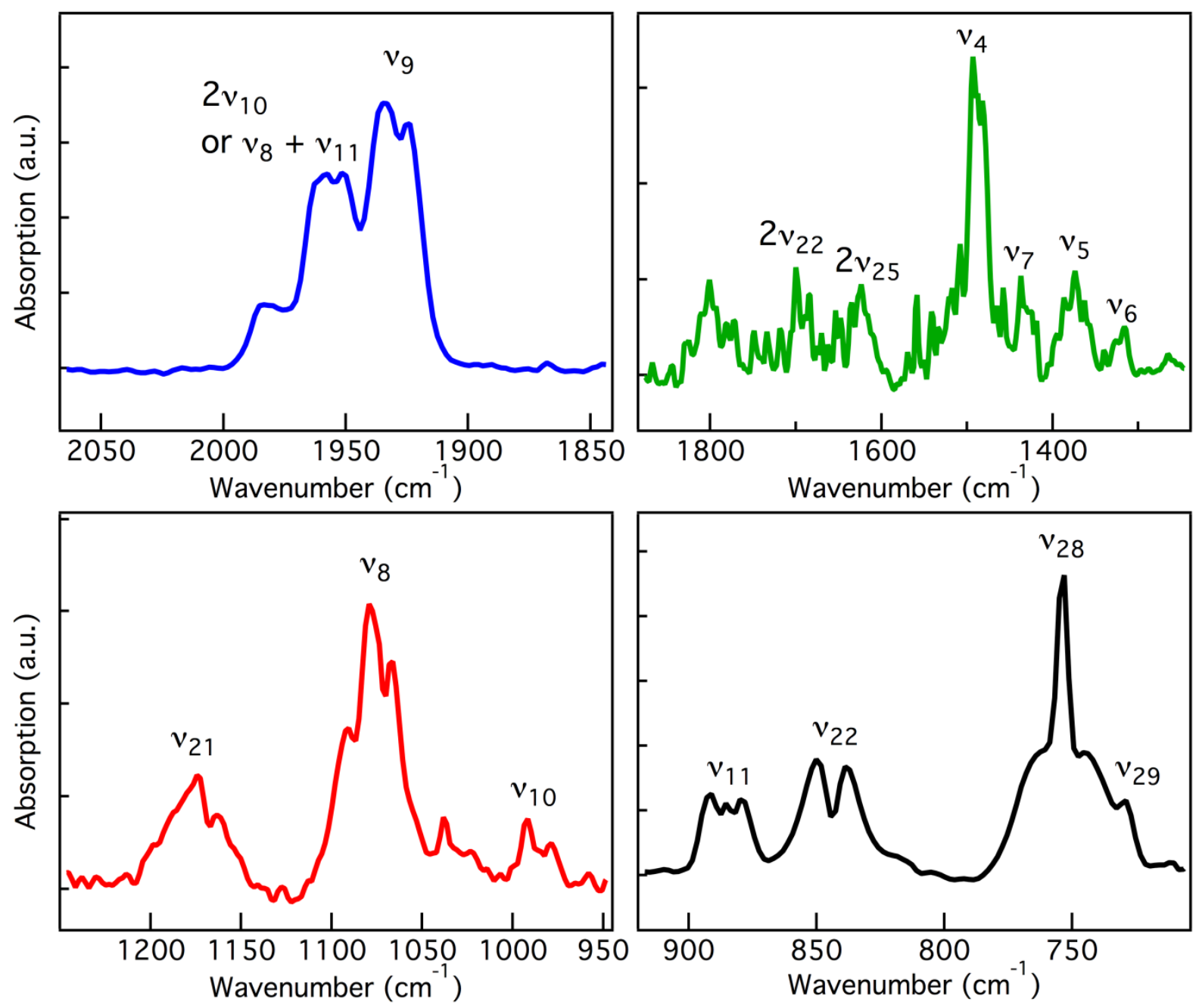

Figure 4. Baseline subtracted IR absorption spectra of the gas mixture recorded with 4 $\mathrm{cm}^{-1}$ resolution

Figure 5 displays the absorption spectrum of the gaseous mixture $(0.75 \%$ of fulvenallene in Helium at 800 Torr) with a $8 \mathrm{~cm}^{-1}$ spectral resolution, together with vibrational assignments according to Angell et al. ${ }^{30}$ The same procedure as for Figure 4 is used to baseline subtract the spectrum in Figure 5. The feature at $1983 \mathrm{~cm}^{-1}$ (labeled $2 v_{10}$ in Figure 5) was identified as coming from fulvenallene, although it was not assigned to a specific vibrational mode. ${ }^{30}$ The 
observed fundamental vibrational modes of fulvenallene are also in agreement with the simulated spectrum obtained using the B3LYP/CBSB7 method.

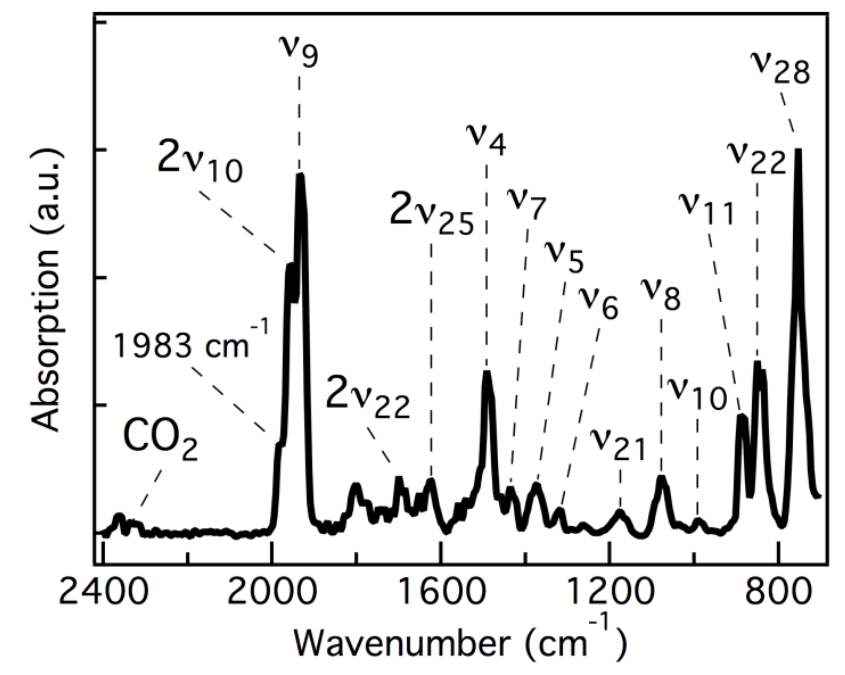

Figure 5. Baseline subtracted absorption spectrum with $8 \mathrm{~cm}^{-1}$ resolution of the synthesized liquid vapors in helium. The fulvenallene vibrational bands are assigned according to Angell et al. ${ }^{30}$

Table 2 shows the vibrational assignments used in Figures 4 and 5. The rotational structures observed in Figure 4 are all in agreement with the measurements by Angell et al. ${ }^{30}$ 
Table 2. Vibrational assignments of the observed gas phase fulvenallene absorption bands

\begin{tabular}{|c|c|c|c|}
\hline Mode & Assignment & Observed $\left(\mathrm{cm}^{-1}\right)$ & Angell $1971\left(\mathrm{~cm}^{-1}\right)$ \\
\hline $\mathrm{A}_{1} v_{1}$ & CH stretching & & 3135 \\
\hline $\mathrm{A}_{1} v_{2}$ & $\mathrm{CH}$ stretching & & 3092 \\
\hline $\mathrm{A}_{1} v_{3}$ & $\mathrm{CH}_{2}$ sym. stretching & & 2966 \\
\hline $\mathrm{A}_{1} v_{4}$ & $\mathrm{C}=\mathrm{C}$ sym. stretching & 1488 & 1486 \\
\hline $\mathrm{A}_{1} v_{5}$ & $\mathrm{CH}$ bending & 1372 & 1362 \\
\hline $\mathrm{A}_{1} v_{6}$ & $\mathrm{CH}$ bending & 1320 & 1320 \\
\hline $\mathrm{A}_{1} v_{7}$ & $\mathrm{CH}_{2}$ scissoring & 1435 & 1432 \\
\hline $\mathrm{A}_{1} v_{8}$ & $\mathrm{C}=\mathrm{C}=\mathrm{C}$ sym. stretching & 1074 & 1073 \\
\hline $\mathrm{A}_{1} v_{9}$ & $\mathrm{C}=\mathrm{C}=\mathrm{C}$ asym. stretching & 1935 & 1935 \\
\hline $\mathrm{A}_{1} v_{10}$ & C-C stretching & 991 & 985 \\
\hline $\mathrm{A}_{1} v_{11}$ & $\mathrm{C}-\mathrm{C}-\mathrm{C}$ stretching & 887 & 886 \\
\hline $\mathrm{A}_{1} v_{12}$ & Ring breathing & & \\
\hline $\mathrm{A}_{2} v_{13}$ & $\mathrm{CH}_{2}$ twisting & & \\
\hline $\mathrm{A}_{2} v_{14}$ & $\mathrm{CH}$ bending & & \\
\hline $\mathrm{A}_{2} v_{15}$ & $\mathrm{CH}$ bending & & \\
\hline $\mathrm{A}_{2} v_{16}$ & Ring bending & & \\
\hline $\mathrm{B}_{1} v_{17}$ & CH stretching & & 3122 \\
\hline $\mathrm{B}_{1} v_{18}$ & CH stretching & & 3071 \\
\hline $\mathrm{B}_{1} v_{19}$ & $\mathrm{C}=\mathrm{C}$ asym. stretching & 1653 & 1655 \\
\hline $\mathrm{B}_{1} v_{20}$ & $\mathrm{CH}$ bending & & \\
\hline $\mathrm{B}_{1} v_{21}$ & CH bending & 1174 & 1170 \\
\hline $\mathrm{B}_{1} v_{22}$ & $\mathrm{CH}_{2}$ wagging & 846 & 844 \\
\hline $\mathrm{B}_{1} v_{23}$ & $\mathrm{C}=\mathrm{C}=\mathrm{C}$ bending & & \\
\hline $\mathrm{B}_{1} v_{24}$ & $\mathrm{C}=\mathrm{C}=\mathrm{C}$ waging & & \\
\hline $\mathrm{B}_{1} v_{25}$ & $\mathrm{C}-\mathrm{C}-\mathrm{C}$ stretching & 812 & 810 \\
\hline $\mathrm{B}_{1} v_{26}$ & Ring bending & & 530 \\
\hline $\mathrm{B}_{2} v_{27}$ & $\mathrm{CH}_{2}$ asym. stretching & & 3010 \\
\hline $\mathrm{B}_{2} v_{28}$ & $\mathrm{CH}$ bending & 753 & 754 \\
\hline $\mathrm{B}_{2} v_{29}$ & CH bending & 729 & 733 \\
\hline $\mathrm{B}_{2} v_{30}$ & $\mathrm{CH}_{2}$ rocking & & 604 \\
\hline $\mathrm{B}_{2} v_{31}$ & $\mathrm{C}=\mathrm{C}=\mathrm{C}$ bending & & \\
\hline $\mathrm{B}_{2} v_{32}$ & $\mathrm{C}=\mathrm{C}=\mathrm{C}$ rocking & & \\
\hline \multirow[t]{4}{*}{$\mathrm{B}_{2} v_{33}$} & Ring bending & & \\
\hline & $2 v_{10}$ or $v_{8}+v_{11}$ & 1958 & 1958 \\
\hline & $2 v_{22}$ & 1700 & 1700 \\
\hline & $2 v_{25}$ & 1622 & 1623 \\
\hline
\end{tabular}


Geometry optimization and vibrational frequencies calculations were carried out using the Gaussian09 package, using the B3LYP/CBSB7 method. All the vibrational frequencies are real, indicating that the optimized geometries represent minima on the potential energy surface. Figure 6 compares (a) the simulated spectrum of fulvenallene with (b) the spectrum of the gaseous mixture. The simulated spectra are scaled by a factor 0.952 to match the observed normal modes wavenumbers of fulvenallene. Over the experimental wavenumber range, all the normal modes of fulvenallene are observed. The difference between the calculated and experimental spectrum is mostly due to overtone and combination vibrational bands ${ }^{30}$ (see Table 3).

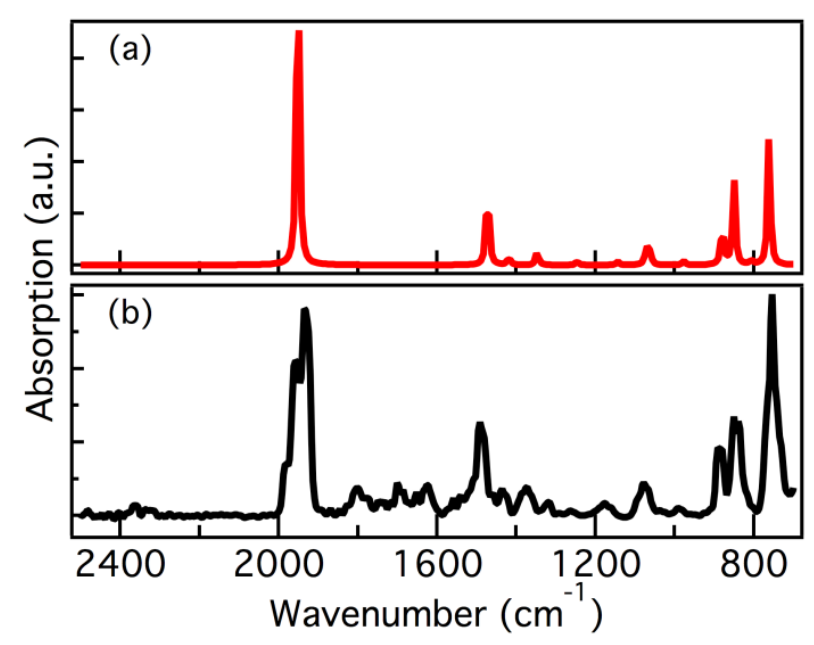

Figure 6. (a) Simulated spectrum (B3LYP/CBSB7 method) of fulvenallene and (b) baseline subtracted absorption spectrum of the gas mixture. The calculated spectrum is scaled by a factor 0.952 to match the observed normal modes of fulvenallene.

\subsubsection{Fulvenallene Purity}

In Figure 5, the absorption band at $1808 \mathrm{~cm}^{-1}$ may be attributed to the presence of impurities in the gas sample. Figure 7 displays the IR spectrum of homophthalic anhydride and 
benzocyclobutenone, obtained using the B3LYP/CBSB7 method. By comparison with liquid phase (NIST) and simulated spectra the observed band is likely to be due to the presence of small quantities of carbonyl containing molecules such as homophthalic anhydride and benzocyclobutenone.
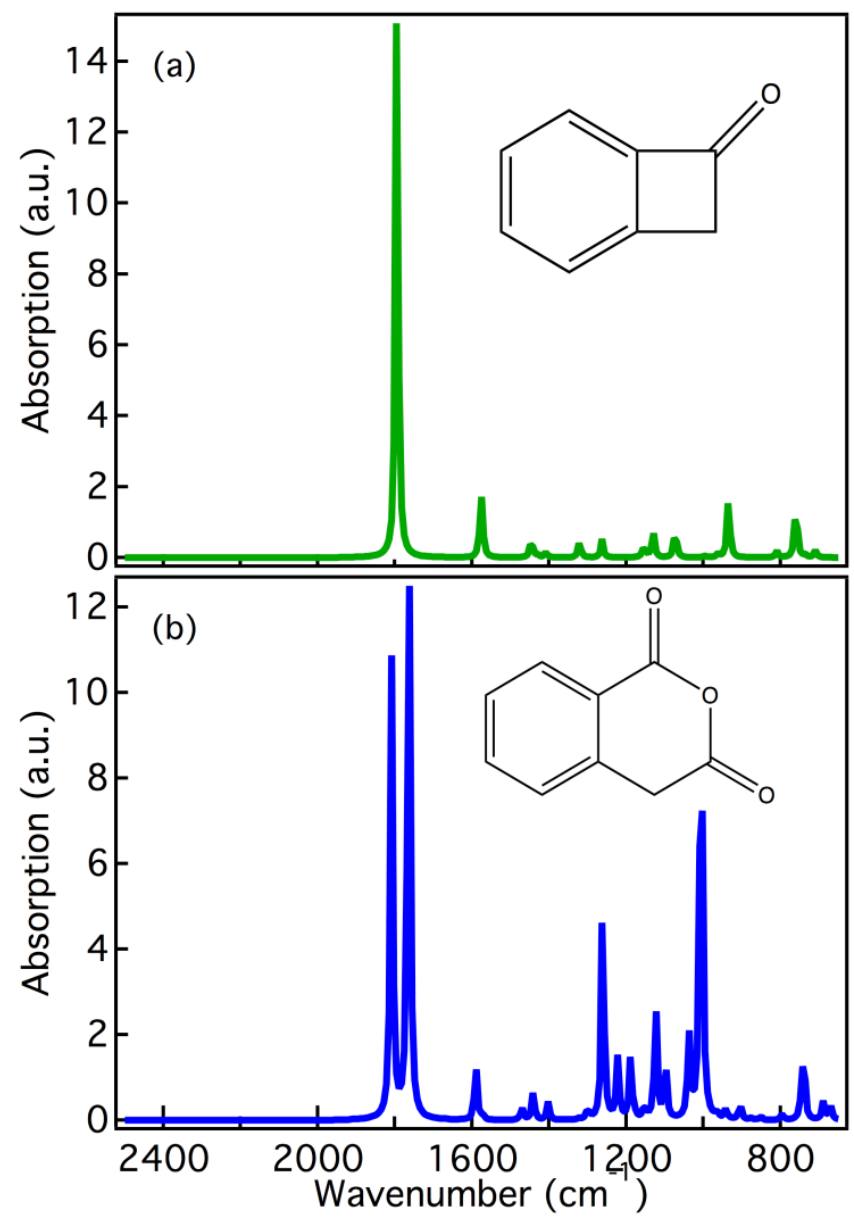

Figure 7. Simulated absorption spectra of homophthalic anhydride and benzocyclobutenone using the B3LYP/CBSB7 method. The calculated spectra are scaled by a factor 0.952 .

Figure 8 displays routine IR absorption spectra of one of the gas mixtures used for the kinetic experiments. Additional absorption bands are observed at $1270 \mathrm{~cm}^{-1}, 1037 \mathrm{~cm}^{-1}$ and 820 
$\mathrm{cm}^{-1}$. Based on the comparison with the simulated spectrum from Figure 7 , the additional absorption bands are likely to come from the presence of homophthalic anhydride.

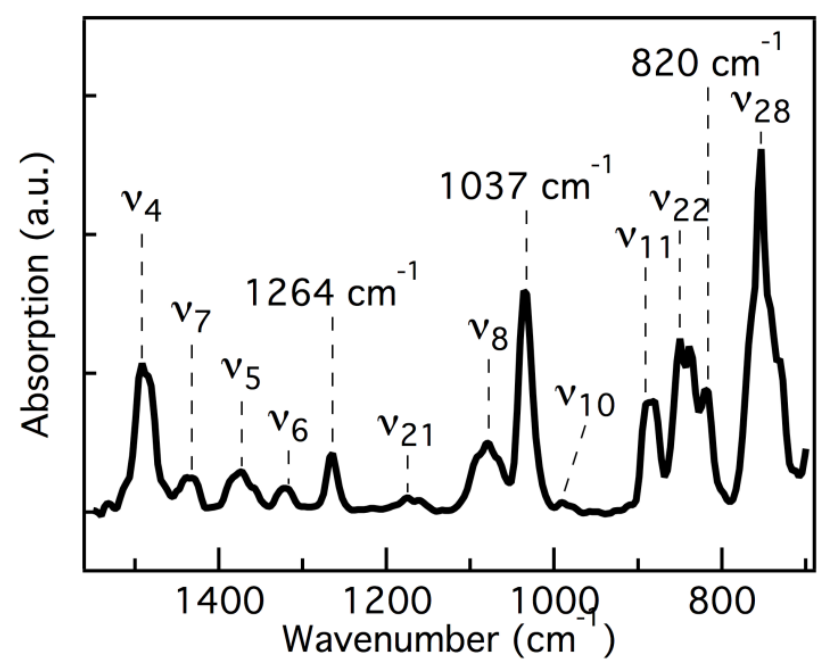

Figure 8. Baseline subtracted absorption spectrum $\left(8 \mathrm{~cm}^{-1}\right.$ resolution) of a gaseous sample, showing additional absorption bands at $1264 \mathrm{~cm}^{-1}, 1037 \mathrm{~cm}^{-1}$ and $820 \mathrm{~cm}^{-1}$.

The purity of gaseous fulvenallene samples was estimated from the ratio of the band maximum normalized by the computed oscillator strength following the equation:

$\frac{\varepsilon_{1}\left(\tilde{v}_{1}\right)}{\varepsilon_{2}\left(\tilde{v}_{2}\right)}=\frac{y_{1}}{y_{2}} \frac{f\left(\tilde{v}_{1}\right)}{f\left(\tilde{v}_{2}\right)}=\frac{y_{1}}{y_{2}} \frac{\tilde{v}_{1}}{\tilde{v}_{2}} \frac{D_{1}}{D_{2}}$

where $v_{1}$ and $v_{2}$ are proportional to the absorption from molecules 1 and 2 at wavenumbers $\tilde{v}_{1}$ and $\tilde{v}_{2}, f_{1}$ and $f_{2}$ the corresponding oscillator strengths, and $y_{1} / y_{2}$ the ratio of the molar fractions. $D_{1}$ and $D_{2}$ are the dipole strengths obtained from the B3LYP/CBSB7 method. Equation 1 assumes similar broadening for both absorption bands. Table 3 displays the wavenumber and computed dipole strengths $D_{i}$ for fulvenallene and homophthalic anhydride. The measured 
absorbance values are from two different spectra. Spectrum 1 is displayed in Figure 5 of the manuscript while spectrum 2 is displayed in Figure 8.

Table 3. Wavenumber, calculated dipole strength, and measured intensity for fulvenallene and homophthalic absorption bands. The ratio of the molar fractions is calculated using equation 2.

\begin{tabular}{lccccc}
\hline & Molecule & $\tilde{v}\left(\mathrm{~cm}^{-1}\right)$ & $\begin{array}{c}D \\
\left(10^{-40} \mathrm{esu}^{2} \mathrm{~cm}^{2}\right)\end{array}$ & $\begin{array}{c}\text { Measured } \\
\text { intensity }\end{array}$ & $y_{1} / y_{2}$ \\
\hline \multirow{2}{*}{ Spectrum 1 } & Fulvenallene & 1935 & 408.17 & 0.56 & 0.06 \\
& homophthalic anhydride & 1808 & 952.89 & 0.07 & 0.16 \\
\hline \multirow{2}{*}{ Spectrum 2 } & Fulvenallene & 1492 & 130.23 & 0.09 \\
\hline
\end{tabular}

Absorption spectra were not recorded for all of the samples used for the kinetics measurements. Figures 5 and 8 are characteristic of the purest and most contaminated recorded samples, respectively. Based on these two spectra, the purity of the fulvenallene gas samples used for the kinetics study are estimated to be between $90 \%$ and $94 \%$.

\subsection{Kinetic Measurements}

\subsubsection{Fulvenallene Number Density}

The fraction of gaseous fulvenallene collected in the $2 \mathrm{~L}$ cylinders stored under pressurized helium buffer gas was used to determine the number density of fulvenallene in all the experiments by using the following equation 3 .

$$
n_{F a}=\frac{q_{F a}}{Q_{F a}} \cdot y_{F a^{-}} \frac{P}{k T}
$$

Where $\boldsymbol{n}_{\boldsymbol{F a}}$ is the number density of fulvenallene in the reaction chamber, $\boldsymbol{q}_{\boldsymbol{F a}}$ is the volumetric flow rate of fulvenallene through the calibrated mass flow controllers, $Q_{F a}$ is the total volumetric 
flow rate of fulvenallene and the helium buffer gas, $y_{F a}$ is the fraction of fulvenallene in the $2 \mathrm{~L}$ cylinders used for all the experiments, $P$ is the pressure of the experiment in the reaction chamber, $k$ is the Boltzmann constant and $T$ is the temperature at which the experiment were conducted in Kelvin.

\subsubsection{Pseudo-First Order Kinetics}

Within the pseudo first order approximation, corresponding to fulvenallene (PAH precursor) being in large excess relative to the $\mathrm{OH}$ radical, the $\mathrm{OH}$ decay signal due to its reaction with fulvenallene can be expressed using the following equations:

$n_{\text {OH }}=n_{O H}^{0} \exp \left(-k_{1 s t} t\right)$

where $\boldsymbol{n}_{\boldsymbol{O H}}$ and $\boldsymbol{n}_{O H}^{0}$ are the $\mathrm{OH}$ radical number densities at delaytime $t$ and $t=0$, respectively. The pseudo-first order rate coefficient $\boldsymbol{k}_{\mathrm{r}^{\mathrm{t}}}$ is given by:

$k_{\mathrm{1}^{t}}=k_{2^{n d}} n_{F A}+k_{\mathrm{1}^{t}}^{0}$

where $k_{2^{n d}}$ is the second order rate coefficient for the $\mathrm{OH}+$ fulvenallene reaction, $n_{F A}$ is the fulvenallene number density, and $\boldsymbol{k}_{\mathrm{r}^{t}}^{0}$ is the first order rate constant due to $\mathrm{OH}$ diffusion and $\mathrm{OH}$ reaction with its precursor. The integrated LIF signal is plotted against delay time for a known reactant number density and fitted using equation 1 to determine the corresponding $k_{1 s t}$.

Figure 9(a) displays the $\mathrm{OH}$ radical decay measured by laser-induced fluorescence at 282 nm following the pulsed-laser photolysis of $\mathrm{H}_{2} \mathrm{O}_{2}$ in the presence of fulvenallene with a calculated number density of $4.29 \times 10^{13} \mathrm{~cm}^{-3}$ and (b) the pseudo-first order decay rate as a function of fulvenallene number density at $298 \mathrm{~K}$ and 5 Torr. The integrated LIF signals are 
fitted using equation 4 to determine the corresponding $k_{\mathrm{r}^{t}}$. Figure 9(b) shows a typical plot of $k_{1 s t}$ vs. concentration of reactant (fulvenallene), for one data set at room temperature. The slope of the linear fit is equal to the second order rate constant $\left(k_{2 n d}\right)$. The uncertainties in Figure 9(b) are $2 \sigma$ from the least-squares regression. Due to the low fulvenallene number density, the change in $\mathrm{OH}$ decay rate with increasing reactant concentration is relatively small. Nonetheless, the $\boldsymbol{k}_{\mathrm{r}^{t}}$ increase observed in Figure 9 is statistically significant and leads to a rate coefficient of $9.43( \pm 1.42) \times 10^{-12} \mathrm{~cm}^{3} \mathrm{~s}^{-1}$, where the error is $2 \sigma$ from the linear fit. The main source of error on the measurements of $\boldsymbol{k}_{2^{n d}}$ is the purity of the fulvenallene gas sample. In order to account for these uncertainties, the rate coefficients are measured using at least three independent experiments, each using different fulvenallene samples. The reaction rate coefficient obtained by averaging three measurements is $8.75( \pm 1.64) \times 10^{-12} \mathrm{~cm}^{3} \mathrm{~s}^{-1}$ at $298 \mathrm{~K}$. The error is $1 \sigma$ around the mean value. The rate coefficient is found to be independent of cell pressure over the 5-10 Torr range. 

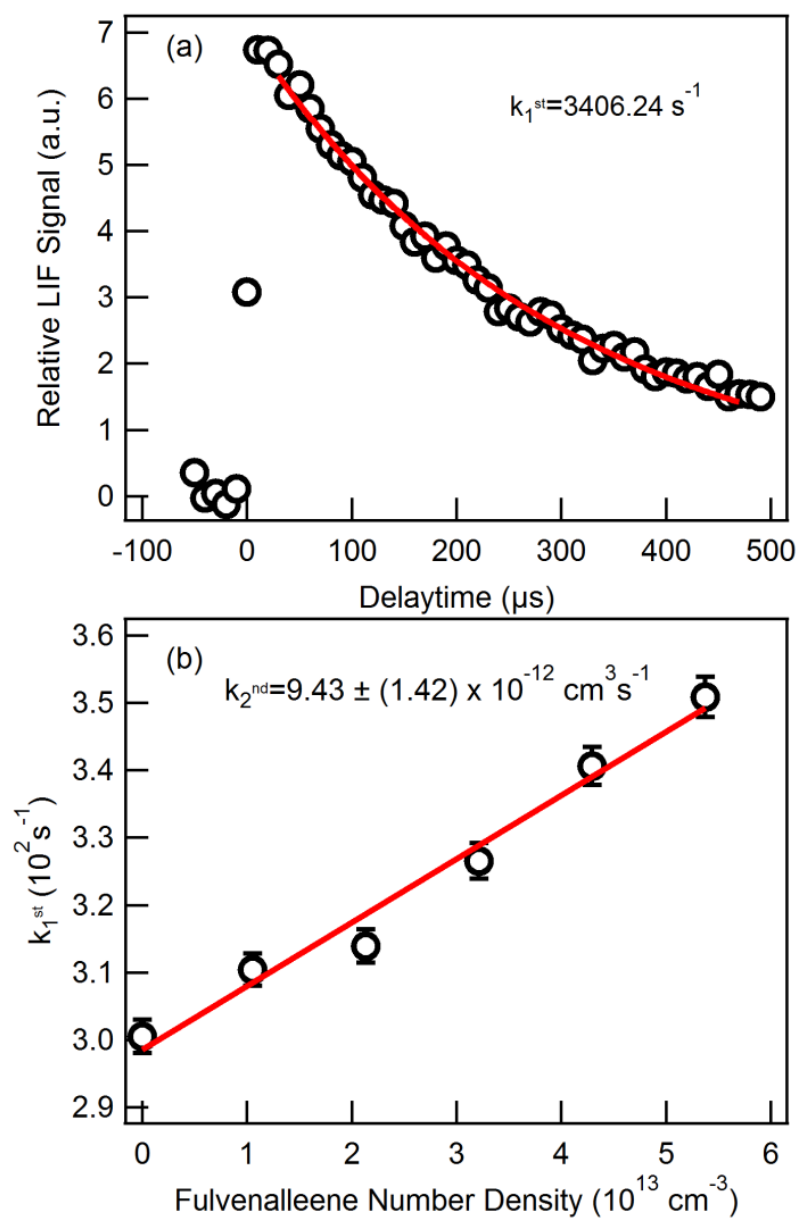

Figure 9 (a) Temporal profile of the $\mathrm{OH}$ radical and (b) pseudo first-order decay as a function of fulvenallene number density recorded at $298 \mathrm{~K}$ and 5 Torr. The red lines are (a) an exponential and (b) a linear fit to the data. The error bars are $2 \sigma$ from the least-squared exponential fits.

\subsubsection{Temperature Dependence of the Rate Coefficient}

The collected fulvenallene sample was employed to measure the temperature dependence of the $\mathrm{OH}+$ fulvenallene reaction rate coefficient from $298 \mathrm{~K}$ to $450 \mathrm{~K}$ at 5 Torr in helium buffer gas. Table 4 displays all the temperatures of the experiments, number density of fulvenallene and 
rate coefficient values of $\mathrm{OH}+$ fulvenallene reaction, the average values of $\mathrm{k}_{2 \mathrm{nd}}$ and their average at the corresponding temperatures.

Table 4. Rate coefficient and fulvenallene number density for single kinetic measurements, and averages values. Symbols refer to the experiments performed with the same gaseous mixture.

\begin{tabular}{|c|c|c|c|c|}
\hline $\begin{array}{l}\text { Temperature } \\
\text { (K) }\end{array}$ & $\begin{array}{c}\text { Number density } \\
\text { range }\left(10^{13} \mathrm{~cm}^{-}\right. \\
3)\end{array}$ & $\begin{array}{c}k_{2 n d} \\
\left(10^{-12} \mathrm{~cm}^{3} \mathrm{~s}^{-1}\right)\end{array}$ & $\begin{array}{l}k_{2 n d} \text { average } \\
\left(10^{-12} \mathrm{~cm}^{3} \mathrm{~s}^{-1}\right)\end{array}$ & $\begin{array}{c}1 \sigma \\
\left(10^{-12} \mathrm{~cm}^{3} \mathrm{~s}^{-1}\right)\end{array}$ \\
\hline 298 & $1.15-7.07$ & $6.88^{\#}$ & & \\
\hline 298 & $1.48-7.54$ & $9.94^{*}$ & 8.8 & 1.7 \\
\hline 298 & $1.05-5.37$ & $9.43^{\circ}$ & & \\
\hline 350 & $1.27-6.46$ & $9.04^{*}$ & & \\
\hline 350 & $0.90-4.61$ & $5.79^{\circ}$ & 5.1 & 3.0 \\
\hline 350 & $1.08-5.52$ & $2.14^{\circ}$ & & \\
\hline 350 & $1.21-7.44$ & $3.38^{*}$ & & \\
\hline$\overline{400}$ & $1.11-5.65$ & $6.13^{*}$ & & \\
\hline 400 & $0.95-6.77$ & $4.25^{\circ}$ & 3.2 & 2.0 \\
\hline 400 & $0.42-5.30$ & $1.93^{\#}$ & & \\
\hline 400 & $1.06-6.51$ & $1.93^{\circ}$ & & \\
\hline 400 & $1.21-7.44$ & $1.50^{\star}$ & & \\
\hline 450 & $0.80-4.93$ & $1.27^{\star}$ & & \\
\hline 450 & $1.95-5.89$ & 2.59 & 1.9 & 0.7 \\
\hline 450 & $2.00-6.04$ & 1.74 & & \\
\hline
\end{tabular}

Figure 10 displays the average $\mathrm{OH}+$ fulvenallene reaction rate coefficients from $298 \mathrm{~K}$ to $450 \mathrm{~K}$, together with the calculated abstraction rate coefficient by da Silva and Bozzelli. ${ }^{19}$ The error bars are $1 \sigma$ around the mean value, and are obtained by averaging at least three independent data sets, all of which were obtained using different fulvenallene gas samples. Because of the uncertainty of the purity of the gaseous samples, the error in the absolute values of the rate coefficient is larger than reported by the error bars in Figure 10. Nonetheless, reaction rates 
measured at different temperatures with the same fulvenallene sample systematically shows a negative temperature dependence. Within the 298-450 K temperature range, the average data can be fit by the expression (red line in Figure 10).

$$
\mathrm{k}(\mathrm{T})=8.8( \pm 1.7) \times 10^{-12}\left(\frac{\mathrm{T}}{298 \mathrm{~K}}\right)^{-6.6( \pm 1.1)} \exp \left(-\frac{8.72( \pm 3.03) \mathrm{kJ}}{\mathrm{R}}\left(\frac{1}{\mathrm{~T}}-\frac{1}{298 \mathrm{~K}}\right)\right) \mathrm{cm}^{3} \mathrm{~s}^{-1}
$$

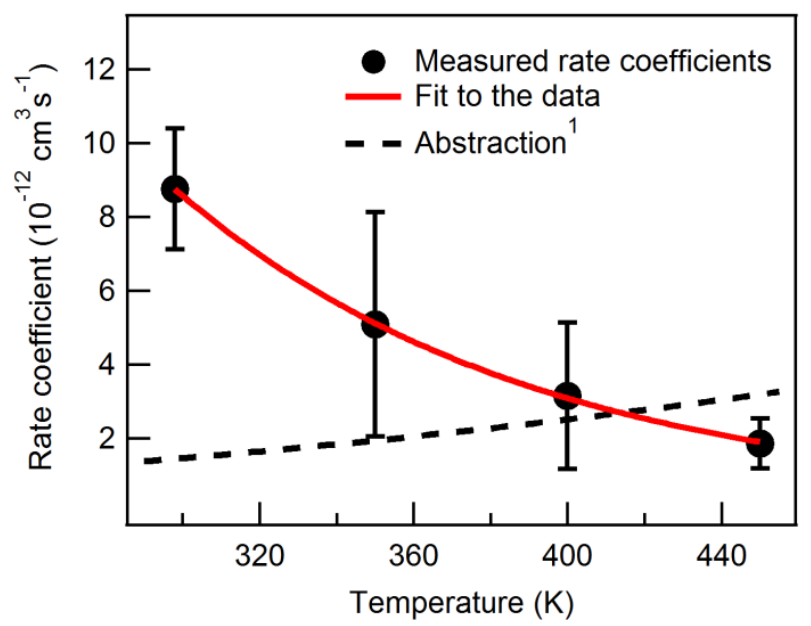

Figure 10. Second-order rate coefficients $\left(\mathrm{k}_{2 \mathrm{nd}}\right)$ as a function of reaction cell temperature from 298 to $450 \mathrm{~K}$. Each data point is averaged $\mathrm{k}_{2 \mathrm{nd}}$ with $1 \sigma$ precision values from multiple runs ( 3 to 5 runs). The red line is the fit to our experimental data and the black dotted line is the abstraction rate constants calculated by da Silva et.al. ${ }^{19}$ as a function of temperature (K).

\subsection{Discussion on the Mechanism}

The negative temperature dependence observed in Figure 10 is likely due to the barrierless formation of a van der Waals complex between the $\mathrm{OH}$ radical and the $\pi$-electron system of fulvenallene as observed for the $\mathrm{OH}+$ allene reaction. The adduct decomposition back to the reactant is likely to become more favorable at high temperatures, leading to an apparent slower rate coefficient. At room temperature, the large rate coefficient (almost one order of 
magnitude larger than that predicted for abstraction) is indicative of a relatively stable adduct which may further isomerize into one of the five $\mathrm{C}_{7} \mathrm{H}_{6}-\mathrm{OH}$ addition isomers. Table 5 displays the optimized product structures and enthalpies for the formation of abstraction and association products calculated using the CBS-QB3 method. The $2-\mathrm{C}_{7} \mathrm{H}_{6} \mathrm{OH}$ isomer is found to be the most stable association product due to the delocalization of the unpaired electron over the entire molecule. In the case of association of the $\mathrm{OH}$ with allene, Zador and Miller predict that the addition is more favorable onto the molecule's central carbon relatively to the terminal carbons. ${ }^{22}$ This information combined with the calculated reaction enthalpies in Table 5, suggests that $2-$ $\mathrm{C}_{7} \mathrm{H}_{6} \mathrm{OH}$ is the most likely product formed by association of the $\mathrm{OH}$ with the $\mathrm{C}=\mathrm{C}=\mathrm{CH}_{2}$ group of fulvenallene at room temperature. Association with the ring could lead to the formation of one of the three ring-addition isomers, with $4-\mathrm{C}_{7} \mathrm{H}_{6} \mathrm{OH}$ being the most stable one. To our knowledge, there is no information available on the interaction of the $\mathrm{OH}$ radical with the cyclopentadiene ring. Without theoretical calculation of the full $\mathrm{C}_{7} \mathrm{H}_{6} \mathrm{OH}$ potential energy surface, it is not possible to predict if the $\mathrm{OH}$ radical will predominantly interact with the allene- or cyclopentadiene-like structure of fulvenallene.

In the case of a long-lived van der Waals intermediate, the competition between formation, decomposition, and isomerization of the initial complex leads to bi-exponential decay of the $\mathrm{OH}$ concentration. ${ }^{31}$ Because of the low fulvenallene concentration in our experiments, it is not possible to make conclusions about the mono- or bi-exponential behavior of the $\mathrm{OH}$ temporal profiles. A non-exponential decay would bias the absolute value of the measured rates and increase the uncertainty of the association rate measurement. As the temperature increases, the van der Waals complex is likely to have a shorter lifetime, leading $\mathrm{OH}$ mono-exponential decays. 
Table 5. Reaction enthalpy for the abstraction and addition channels of $\mathrm{OH}+$ Fulvenallene calculated using the CBSQB3 method.

\begin{tabular}{|c|c|c|c|}
\hline Products & $\begin{array}{c}\Delta \mathrm{H}(298 \mathrm{~K}) \\
\left(\mathrm{KJmol}^{-1}\right)\end{array}$ & Products & $\begin{array}{c}\Delta \mathrm{HH}(298 \mathrm{~K}) \\
\left(\mathrm{KJmol}^{-1}\right)\end{array}$ \\
\hline $\mathrm{H}_{2} \mathrm{O}+1-\mathrm{C}_{7} \mathrm{H}_{5}$ & -157.036 & $2-\mathrm{C}_{7} \mathrm{H}_{6} \mathrm{OH}$ & -277.479 \\
\hline $\mathrm{H}_{2} \mathrm{O}+4-\mathrm{C}_{7} \mathrm{H}_{5}$ & -6.740 & $3-\mathrm{C}_{7} \mathrm{H}_{6} \mathrm{OH}$ & -85.696 \\
\hline $\mathrm{H}_{2} \mathrm{O}+5-\mathrm{C}_{7} \mathrm{H}_{5}$ & -14.485 & $4-\mathrm{C}_{7} \mathrm{H}_{6} \mathrm{OH}$ & -172.842 \\
\hline $1-\mathrm{C}_{7} \mathrm{H}_{6} \mathrm{OH}$ & -97.104 & $5-\mathrm{C}_{7} \mathrm{H}_{6} \mathrm{OH}$ & -145.917 \\
\hline
\end{tabular}

At $450 \mathrm{~K}$ the measured rate is similar to that predicted for abstraction of the $-\mathrm{CH}-\mathrm{H}$ hydrogen suggesting rapid dissociation of the $\mathrm{OH}-$ fulvenallene adduct back to the reactants. At this temperature and higher, $\mathrm{C}_{7} \mathrm{H}_{5}$-formation through water elimination is likely to be the dominant mechanism. The reaction enthalpies for the abstraction channels displayed in Table 5 are consistent with the weak $-\mathrm{CH}-\mathrm{H}$ fulvenallene bond calculated at a higher level of theory by da Silva and Bozzelli. ${ }^{19}$ Thermodynamically, the formation of the fulvenallene $+\mathrm{H}_{2} \mathrm{O}$ is the most favorable abstraction channel, as displayed in Table 5.

\subsection{Conclusion}

The reaction of hydroxyl radical with fulvenallene was studied over 298-450 K at 5 Torr (667 Pa) using pulsed laser photolysis-laser induced fluorescence (PLP-LIF) technique. The room temperature reaction rate is found to be $8.8( \pm 1.7) \times 10^{-12} \mathrm{~cm}^{3} \mathrm{~s}^{-1}$. The rate coefficients 
measured from 298 to $450 \mathrm{~K}$ show the negative temperature dependence over the experimental range suggesting the formation of barrierless Van der Waals complex between $\mathrm{OH}$ and fulvenallene. The enthalpies for the abstraction and addition intermediates of $\mathrm{OH}+$ fulvenallene using CBS-QB3 method ${ }^{32}$ suggest that the addition product $\left(2-\mathrm{C}_{7} \mathrm{H}_{6} \mathrm{OH}\right)$ is favorable (See table

5). At room temperature, the experimental rate coefficient is larger than the calculated abstraction rate coefficient (see figure 10) suggesting that the formation of fulvenallenyl radical is likely. At $450 \mathrm{~K}$ and higher, the experimental room temperature is comparable with the calculated abstraction rate coefficient (see figure 10) suggesting that the formation of fulvenallenyl radical at combustion environment is likely.

\section{References:}

(1) Hansen, N.; Kasper, T.; Klippenstein, S. J.; Westmoreland, P. R.; Law, M. E.; Taatjes, C. A.; Kohse-Hoeinghaus, K.; Wang, J.; Cool, T. A. Journal of Physical Chemistry A 2007, $111,4081$.

(2) da Silva, G.; Cole, J. A.; Bozzelli, J. W. Journal of Physical Chemistry A 2009, 113, 6111.

(3) Derudi, M.; Polino, D.; Cavallotti, C. Physical Chemistry Chemical Physics 2011, 13, 21308.

(4) Cavallotti, C.; Derudi, M.; Rota, R. Proceedings of the Combustion Institute 2009, 32, 115.

(5) Buckingham, G. T.; Ormond, T. K.; Porterfield, J. P.; Hemberger, P.; Kostko, O.; Ahmed, M.; Robichaud, D. J.; Nimlos, M. R.; Daily, J. W.; Ellison, G. B. Journal of Chemical Physics 2015, 142.

(6) Polino, D.; Famulari, A.; Cavallotti, C. Journal of Physical Chemistry A 2011, 115, 7928.

(7) Zhang, T.; Zhang, L.; Hong, X.; Zhang, K.; Qi, F.; Law, C. K.; Ye, T.; Zhao, P.; Chen, Y. Combustion and Flame 2009, 156, 2071.

(8) Li, Y.; Zhang, L.; Tian, Z.; Yuan, T.; Wang, J.; Yang, B.; Qi, F. Energy \& Fuels 2009, 23, 1473.

(9) Alexiou, A.; Williams, A. Combustion and Flame 1996, 104, 51.

(10) Andrae, J. C. G.; Bjornbom, P.; Cracknell, R. F.; Kalghatgi, G. T. Combustion and Flame 2007, 149, 2.

(11) Bounaceur, R.; Da Costa, I.; Fournet, R.; Billaud, F.; Battin-Leclerc, F. International Journal of Chemical Kinetics 2005, 37, 25.

(12) Dagaut, P.; Pengloan, G.; Ristori, A. Physical Chemistry Chemical Physics 2002, 4, 1846.

(13) Emdee, J. L.; Brezinsky, K.; Glassman, I. Journal of Physical Chemistry 1992, 96, 2151. 
(14) Oehlschlaeger, M. A.; Davidson, D. F.; Hanson, R. K. Combustion and Flame 2006, 147, 195.

(15) Sivaramakrishnan, R.; Tranter, R. S.; Brezinsky, K. Combustion and Flame 2004, 139, 340.

(16) Sivaramakrishnan, R.; Tranter, R. S.; Brezinsky, K. Proceedings of the Combustion Institute 2005, 30, 1165.

(17) Shapero, M.; Cole-Filipiak, N. C.; Haibach-Morris, C.; Neumark, D. M. Journal of Physical Chemistry A 2015, 119, 12349.

(18) Song, Y.; Zheng, X.; Lucas, M.; Zhang, J. Physical Chemistry Chemical Physics 2011, 13, 8296.

(19) da Silva, G.; Bozzelli, J. W. Journal of Physical Chemistry A 2009, 113, 12045.

(20) Kailasanathan, R. K. A.; Thapa, J.; Goulay, F. Journal of Physical Chemistry A 2014, $118,7732$.

(21) Daranlot, J.; Hickson, K. M.; Loison, J.-C.; Mereau, R.; Caralp, F.; Forst, W.; Bergeat, A. Journal of physical chemistry A 2012, 116, 10871.

(22) Zador, J.; Miller, J. A. Proceedings of the Combustion Institute 2015, 35, 181.

(23) Cava, M. P.; Muth, K. Journal of the American Chemical Society 1960, 82, 652.

(24) Spangler, R. J.; Kim, J. H. Tetrahedron Letters 1972, 1249.

(25) Spangler, R. J.; Beckmann, B. G.; Kim, J. H. Journal of Organic Chemistry 1977, 42, 2989.

(26) Sakaizumi, T.; Katoh, F.; Ohashi, O.; Yamaguchi, I. Journal of Molecular Spectroscopy 1993, 159, 112.

(27) Frisch, M. J. T., G. W.; Schlegel, H. B.; Scuseria, G. E.; Robb, M. A.; Cheeseman, J. R.; Scalmani, G.; Barone, V.; Mennucci, B.; Petersson, G.A. et.al. Gaussian 092009

(28) Lee, C. T.; Yang, W. T.; Parr, R. G. Phys. Rev. B 1988, 37, 785.

(29) Becke, A. D. J. Chem. Phys. 1993, 98, 1372.

(30) Angell, C. L. Journal of Molecular Structure 1971, 10, 265.

(31) Bohn, B.; Zetzsch, C. Physical Chemistry Chemical Physics 2012, 14, 13933.

(32) Frish, M. J. T., G.W.; Schlegel, H.B.; Scuseria, G.E.; Robb, M.A.; Cheeseman, J.R.; Scalmani, G.; Barone, V.; Mennucci, B.; Peterson, G.A.; et.al. Gaussian 092009. 


\section{Chapter 5: Conclusion and Future Directions}

Internal combustion engines operate using the energy released from complex chemical transformation of fuel molecules. Due to incomplete combustion in engines the process still emits various unwanted byproducts such as $\mathrm{NO}_{\mathrm{x}}, \mathrm{SO}_{\mathrm{x}}, \mathrm{CO}, \mathrm{CO}_{2}$, unburned hydrocarbons (HCs), and particulate matters (smoke, soot and its precursors-PAHs). The fuel efficiency of modern gasoline engines is only about $14-30 \% .{ }^{1}$ It is therefore important to better understand the fundamental chemistry of combustion intermediates in order to develop combustion strategies with increased engine efficiency and lower pollutants.

Soot is one of the major pollutants and owing to its environmental (e.g., its deposition melting polar ice due to radiative forcing ${ }^{2,3}$ and regional warming by forming brown clouds $^{3}$ ) and health (e.g., eye and skin irritations, ${ }^{4}$ pulmonary effects (respiratory diseases), ${ }^{5}$ etc.) concerns. Soot particles generated by transportation devices such as trucks, cars, planes, ships, etc. or power stations have been drawn much research attention. Its formation mechanism is not well understood. Experimental and modelling research studies suggest that soot forms via condensation or agglomeration of polycyclic aromatic hydrocarbons (PAHs). These molecules are stable in the combustion environment at high temperatures. ${ }^{6}$ Examples of PAHs are naphthalene, anthracene, phenanthrene, pyrene, etc. PAHs also have several health effects such as eye and skin irritation ${ }^{7}$, nausea ${ }^{7}$, vomiting and diarrhea (high concentration) ${ }^{7}$, skin, lung, bladder, and gastrointestinal cancers. ${ }^{8,9}$ These PAHs can either form via the benzene route followed by hydrogen-abstraction-acetylene-addition (HACA) mechanism, ${ }^{6,10,11}$ or via self or cross combination of resonance-stabilized radicals such as cyclopentadienyl, ${ }^{12-15}$ fulvenallenyl, ${ }^{16}$ etc. The resonance stabilized radicals are predicted to be formed from the reaction of radicals 
(e.g. hydroxyl radical) with molecules such as phenylacetylene and fulvenallene, which are observed in combustion environments.

In this dissertation, the reaction of hydroxyl radical was investigated with phenylacetylene and fulvenallene using pulsed laser photolysis-laser induced fluorescence technique (PLP-LIF) - a well-known laser spectroscopic technique to study combustion reactions. For $\mathrm{OH}+$ phenylacetylene reaction, absolute reaction rates coefficients were obtained over the 293 - $423 \mathrm{~K}$ temperature range and 1-7.5 Torr pressure range. The $\mathrm{OH}$ radicals were formed in the gas phase using either $\mathrm{H}_{2} \mathrm{O}_{2}$ at $266 \mathrm{~nm}$ or $\mathrm{HONO}$ at $355 \mathrm{~nm}$. The reaction rates measured as a function of laser fluence at $266 \mathrm{~nm}$ photolysis wavelength were found to be laserpower dependent, while they are laser-power independent at $355 \mathrm{~nm}$. For this reason, all the experiments were performed at $355 \mathrm{~nm}$ photolysis wavelength. The room temperature reaction rate was measured to be several orders of magnitude greater than the values currently used in combustion models and may therefore play a significant role in phenylacetylene removal and formation of oxidation products. ${ }^{18}$ At this wavelength, within the experimental error bars, the reaction rate was found to be independent of pressure and temperature also. The temperature independence of reaction rate, high level calculations (CBS-QB3 method ${ }^{17}$ ) of the entrance pathway exothermicities, and previous theoretical data on the $\mathrm{OH}+$ benzene $^{18}$ and $\mathrm{OH}+$ diacetylene ${ }^{19}$ reactions suggested the formation of an association pre-reactive complex involving the ethynyl $\left(-\mathrm{C}_{2} \mathrm{H}\right)$ group of phenylacetylene. The Van der Waals intermediate is then likely to evolve without or with a small activation energy to a resonantly stabilized addition intermediate, INT-1 (see chapter 3, figure 16).

Due to the importance of resonance-stabilized radicals in combustion, the reaction of $\mathrm{OH}$ radical with fulvenallene was studied over 298-450 K temperature range at 5 Torr using PLP-LIF 
technique and hydrogen peroxide $\left(\mathrm{H}_{2} \mathrm{O}_{2}\right)$ as $\mathrm{OH}$ radical source at $266 \mathrm{~nm}$. This reaction was predicted to form the fulvenallenyl radical. ${ }^{16}$ The room temperature reaction rate was found to be $8.8( \pm 1.7) \times 10^{-12} \mathrm{~cm}^{3} \mathrm{~s}^{-1}$. The rate coefficients measured from 298 to $450 \mathrm{~K}$ showed the negative temperature dependence over the experimental range suggesting the formation of barrierless Van der Waals complex between $\mathrm{OH}$ and fulvenallene. The enthalpies for the abstraction and addition intermediates of $\mathrm{OH}+$ fulvenallene using CBS-QB3 method $^{17}$ suggest that the addition product (2- $\mathrm{C}_{7} \mathrm{H}_{6} \mathrm{OH}$ ) is favorable (See chapter 4 table 5). At room temperature, the experimental rate coefficient is larger than the calculated abstraction rate coefficient (see chapter 4 figure 10) suggesting that the formation of fulvenallenyl radical is likely. At $450 \mathrm{~K}$ and higher, the experimental room temperature is comparable with the calculated abstraction rate coefficient (see chapter 4 figure 10) suggesting that the formation of fulvenallenyl radical at combustion environment is likely.

Studying the $\mathrm{OH}+$ fulvenallene reaction at higher temperatures will help investigate the formation of fulvenallenyl radical. Furthermore, it is also very important to study the self and cross combination of fulvenallenyl radical and other resonance-stabilized radicals to investigate the formation of polycyclic aromatic hydrocarbons. Since it is not possible to heat the six-way cross reaction cell higher than $450 \mathrm{~K}$, due to heating of the optics, higher temperature reactions will be studied in a wall-free subsonic pulsed fast flow reactor. Figure 1 shows the schematic of the high temperature subsonic pulsed fast flow reactor. It consists of a quasi-static heated doublewalled gas reservoir at 100 Torr, a rotating chopper, a laval nozzle and a tube connected to the vacuum pump. The chopper opens for approximately 4-5 ms to provide pulsed flow while the nozzle regulates the instantaneous mass flow rate. Due to expansion within the nozzle the gas cools down. The presence of the vertical shock wave at the exit of the nozzle restores the 
temperature of the gas reservoir and maintains the subsonic flow. This fast flow reactor developed in the laboratory is a prototype that has the temperature range of $300-750 \mathrm{~K}$ and pressure range of 1-10 Torr. The flow velocity for 3-5 milliseconds time is approximately 100 $\mathrm{m} / \mathrm{s}$ for this set up.

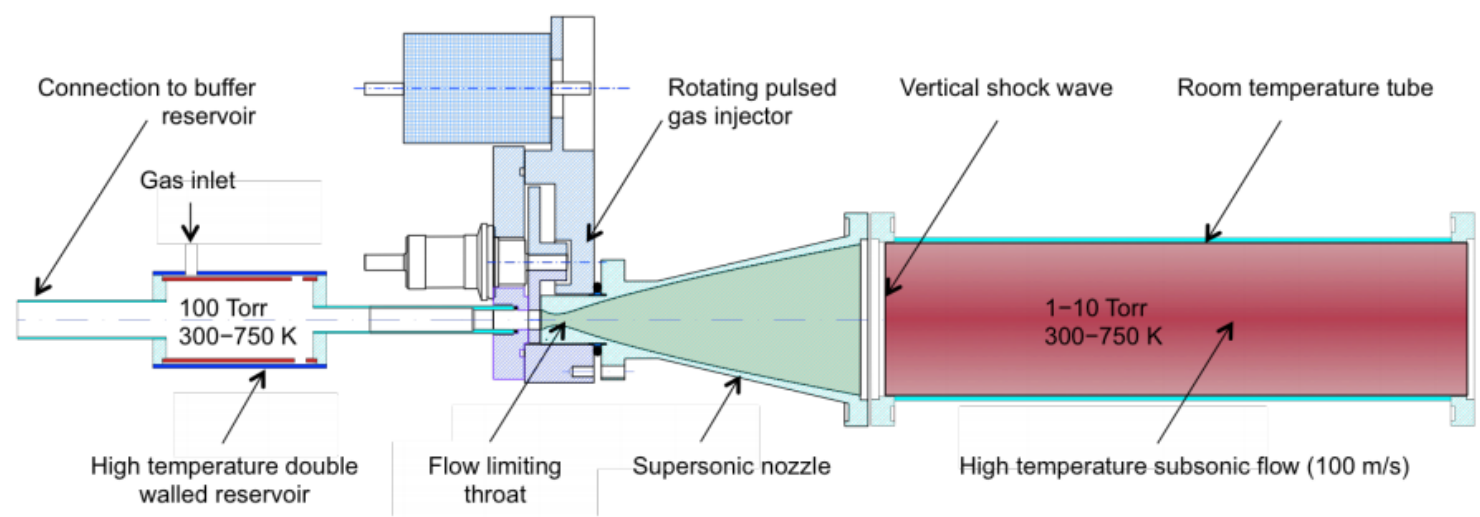

Figure 1. Schematic of high temperature pulsed fast flow reactor

The kinetics experimental set up has a six-way cross arrangement (not shown here) just right to the room temperature tube of the figure 1. The photolysis laser (Nd:YAG) enters opposite the flow of the gas and synchronized with the pulsed chopper at $10 \mathrm{~Hz}$ to generate radicals. The probe laser (tunable dye) enters perpendicular to the photolysis laser that monitors the concentration of the free radicals. Both photolysis and probe lasers enter the reaction chamber through Brewster angle windows. The photomultiplier tube is placed orthogonal to the photolysis and probe lasers to collect the fluorescence signal of free radicals. The fluorescence signal is collected by changing the delay (reaction) time between photolysis and probe lasers using delay generator. This signal is integrated using a boxcar averager and stored in the computer for analysis.

\section{References:}

(1) Where the Energy Goes: Gasoline Vehicles, https://www.fueleconomy.gov/feg/atv.shtml; Department of Energy.

(2) Jacobson, M. Z. Journal of Geophysical Research-Atmospheres 2004, 109. 
(3) Ramanathan, V.; Carmichael, G. Nature Geoscience 2008, 1, 221.

(4) Sydbom, A.; Blomberg, A.; Parnia, S.; Stenfors, N.; Sandstrom, T.; Dahlen, S. E. European Respiratory Journal 2001, 17, 733.

(5) Nel, A. Science 2005, 308, 804.

(6) Frenklach, M. Physical Chemistry Chemical Physics 2002, 4, 2028.

(7) Unwin, J.; Cocker, J.; Scobbie, E.; Chambers, H. Annals of Occupational Hygiene 2006, 50, 395.

(8) Naeher, L. P.; Brauer, M.; Lipsett, M.; Zelikoff, J. T.; Simpson, C. D.; Koenig, J. Q.; Smith, K. R. Inhalation Toxicology 2007, 19, 67.

(9) Smith, K. R.; Frumkin, H.; Balakrishnan, K.; Butler, C. D.; Chafe, Z. A.; Fairlie, I.; Kinney, P.; Kjellstrom, T.; Mauzerall, D. L.; McKone, T. E.; McMichael, A. J.; Schneider, M. In Annual Review of Public Health, Vol 34; Fielding, J. E., Ed. 2013; Vol. 34, p 159.

(10) Miller, J. A.; Pilling, M. J.; Troe, E. Proceedings of the Combustion Institute 2005, 30, 43.

(11) Richter, H.; Howard, J. B. Progress in Energy and Combustion Science 2000, 26, 565.

(12) Cavallotti, C.; Polino, D. Proceedings of the Combustion Institute 2013, 34, 557.

(13) Knyazev, V. D.; Popov, K. V. Journal of Physical Chemistry A 2015, 119, 7418.

(14) Mebel, A. M.; Kislov, V. V. Journal of Physical Chemistry A 2009, 113, 9825.

(15) Melius, C. F.; Colvin, M. E.; Marinov, N. M.; Pitz, W. J.; Senkan, S. M. Reaction mechanisms in aromatic hydrocarbon formation involving the C5H5 cyclopentadienyl moiety, 1996.

(16) da Silva, G.; Bozzelli, J. W. Journal of Physical Chemistry A 2009, 113, 12045.

(17) Frish, M. J. T., G.W.; Schlegel, H.B.; Scuseria, G.E.; Robb, M.A.; Cheeseman, J.R.; Scalmani, G.; Barone, V.; Mennucci, B.; Peterson, G.A.; et.al. Gaussian 092009.

(18) Hollman, D. S.; Simmonett, A. C.; Schaefer, H. F. Physical Chemistry Chemical Physics 2011, 13, 2214.

(19) Senosiain, J. P.; Klippenstein, S. J.; Miller, J. A. Proceedings of the Combustion Institute 2007, 31, 185. 


\section{Appendix}

The total energy, lowest frequencies, and optimized coordinates for fulvenallene using 6$31+\mathrm{G}(\mathrm{d}, \mathrm{p})$ basis sets.

Total Energy $(6-31+G(d))=-270.268552602$ A.U.

Lowest three frequencies $=138.5,157.8,376.4 \mathrm{~cm}^{-1}$

Optimized coordinates $(6-31+\mathrm{G}(\mathrm{d}))$ :
$\mathrm{H} \quad-0.115060 \quad-0.083823 \quad 0.082525$
C $\quad-0.142466 \quad-0.055764 \quad 1.171253$
H $\quad 0.814103 \quad-0.083823 \quad 1.691882$
C $\quad-1.271213 \quad 0.008658 \quad 1.822935$
C $\quad-2.416265 \quad 0.074173 \quad 2.484031$
$\begin{array}{llll}\text { C } & -3.129571 & 1.300019 & 2.895859\end{array}$
$\begin{array}{llll}\text { C } & -3.231591 & -1.063569 & 2.954760\end{array}$
$\begin{array}{llll}\text { C } & -4.321818 & -0.551464 & 3.584202\end{array}$
$\begin{array}{llll}\mathrm{H} & -2.971212 & -2.103525 & 2.804430\end{array}$
$\begin{array}{llll}\mathrm{H} & -5.120268 & -1.122724 & 4.045188\end{array}$
$\begin{array}{llll}\text { C } & -4.258498 & 0.915717 & 3.547645\end{array}$
$\begin{array}{llll}\mathrm{H} & -2.780750 & 2.304841 & 2.694466\end{array}$
$\begin{array}{llll}\mathrm{H} & -5.003798 & 1.575824 & 3.977944\end{array}$ 
Table 1 and Figures 1 to 6 display the results from the ${ }^{1} \mathrm{H}$ and ${ }^{13} \mathrm{C}$ NMR analysis, confirming the synthesis of fulvenallene.

Table 1. Experimental and calculated ${ }^{1} \mathrm{H}$ shift (top table), coupling constants (middle table), and ${ }^{13} \mathrm{C}$ shifts (bottom table) of fulvenallene in $\mathrm{CDCl}_{3}$.

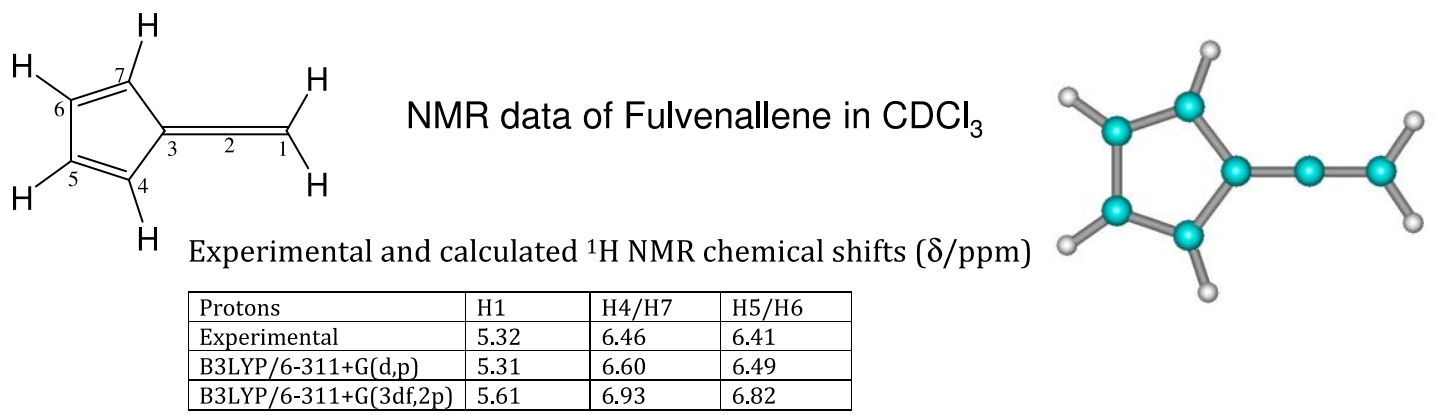

Experimental and calculated coupling constants $(\mathrm{J}(\mathrm{Hz})$

\begin{tabular}{|c|c|c|c|}
\hline $\begin{array}{c}\text { Coupling constants } \\
(\mathrm{J} / \mathrm{Hz})\end{array}$ & Experimental & B3LYP/6-311+G(d,p) & B3LYP/6-311+G(3df,2p) \\
\hline 5 J(H1-H4) & 0.29 & 0.31 & 0.36 \\
\hline${ }^{5} \mathrm{~J}(\mathrm{H} 1-\mathrm{H} 7)$ & 0.29 & 0.31 & 0.36 \\
\hline${ }^{6} \mathrm{~J}(\mathrm{H} 1-\mathrm{H} 5)$ & 1.43 & 2.1 & 2.1 \\
\hline${ }^{6} \mathrm{~J}(\mathrm{H} 1-\mathrm{H} 6)$ & 1.43 & 2.1 & 2.1 \\
\hline${ }^{4} \mathrm{~J}(\mathrm{H} 4-\mathrm{H} 5)$ & 5.17 & 5.84 & 5.87 \\
\hline${ }^{3} \mathrm{~J}(\mathrm{H} 4-\mathrm{H} 6)$ & 1.45 & 0.94 & 1.05 \\
\hline${ }^{4} \mathrm{~J}(\mathrm{H} 4-\mathrm{H} 7)$ & 1.94 & 2.12 & 2.21 \\
\hline${ }^{3} \mathrm{~J}(\mathrm{H} 5-\mathrm{H} 6)$ & 2.06 & 2.54 & 2.58 \\
\hline
\end{tabular}

Experimental and calculated ${ }^{13} \mathrm{C}$ NMR chemical shifts $(\delta / \mathrm{ppm})$

\begin{tabular}{|l|l|l|l|l|l|}
\hline Carbons & C1 & C2 & C3 & C4/C7 & C5/C6 \\
\hline Experimental & 76.08 & 212.48 & 111.17 & 125.17 & 130.30 \\
\hline B3LYP/6-311+G(d,p) & 76.85 & 228.61 & 118.58 & 133.04 & 137.47 \\
\hline B3LYP/6-311+G(3df,2p) & 77.05 & 229.11 & 119.51 & 133.59 & 138.06 \\
\hline
\end{tabular}




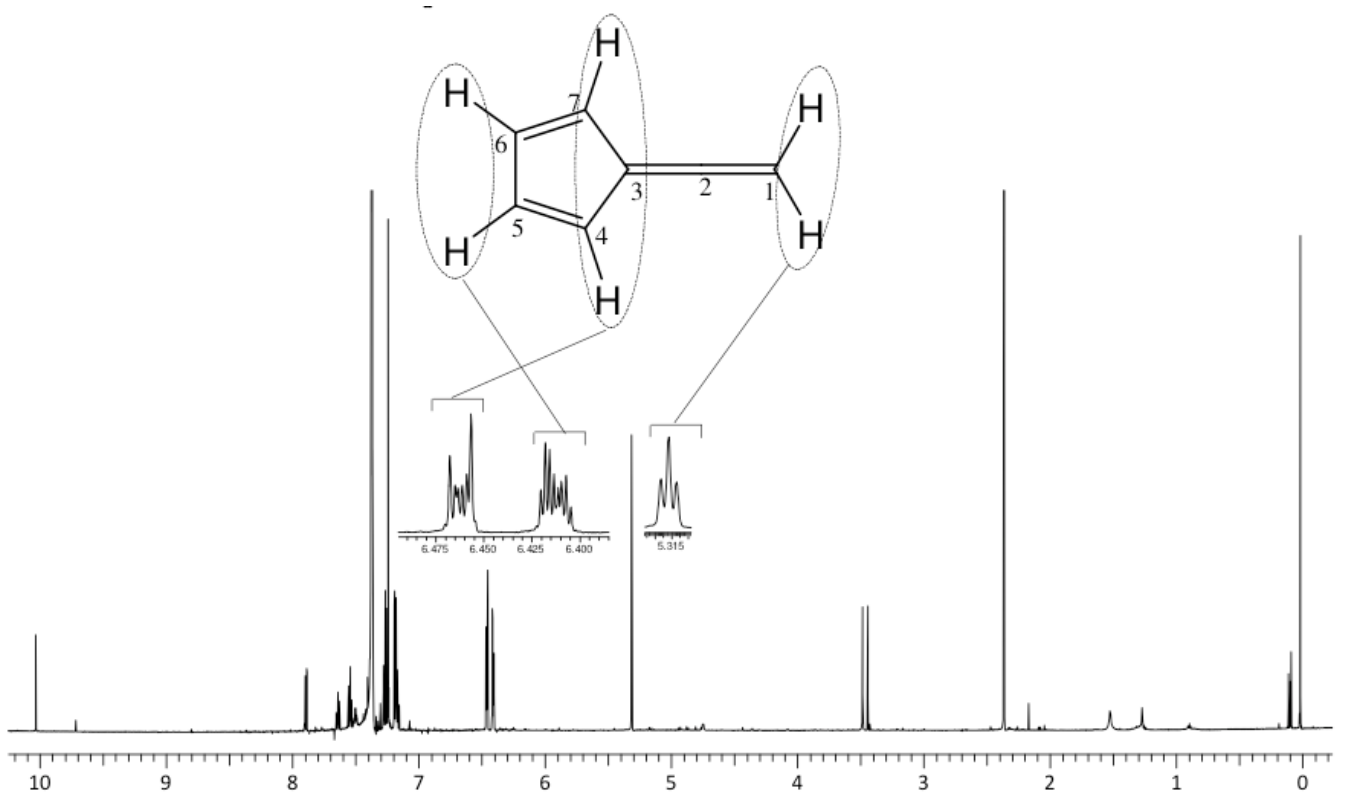

Figure 1. ${ }^{1} \mathrm{H}$ NMR spectrum of the liquid product dissolved in $\mathrm{CDCl}_{3}$.

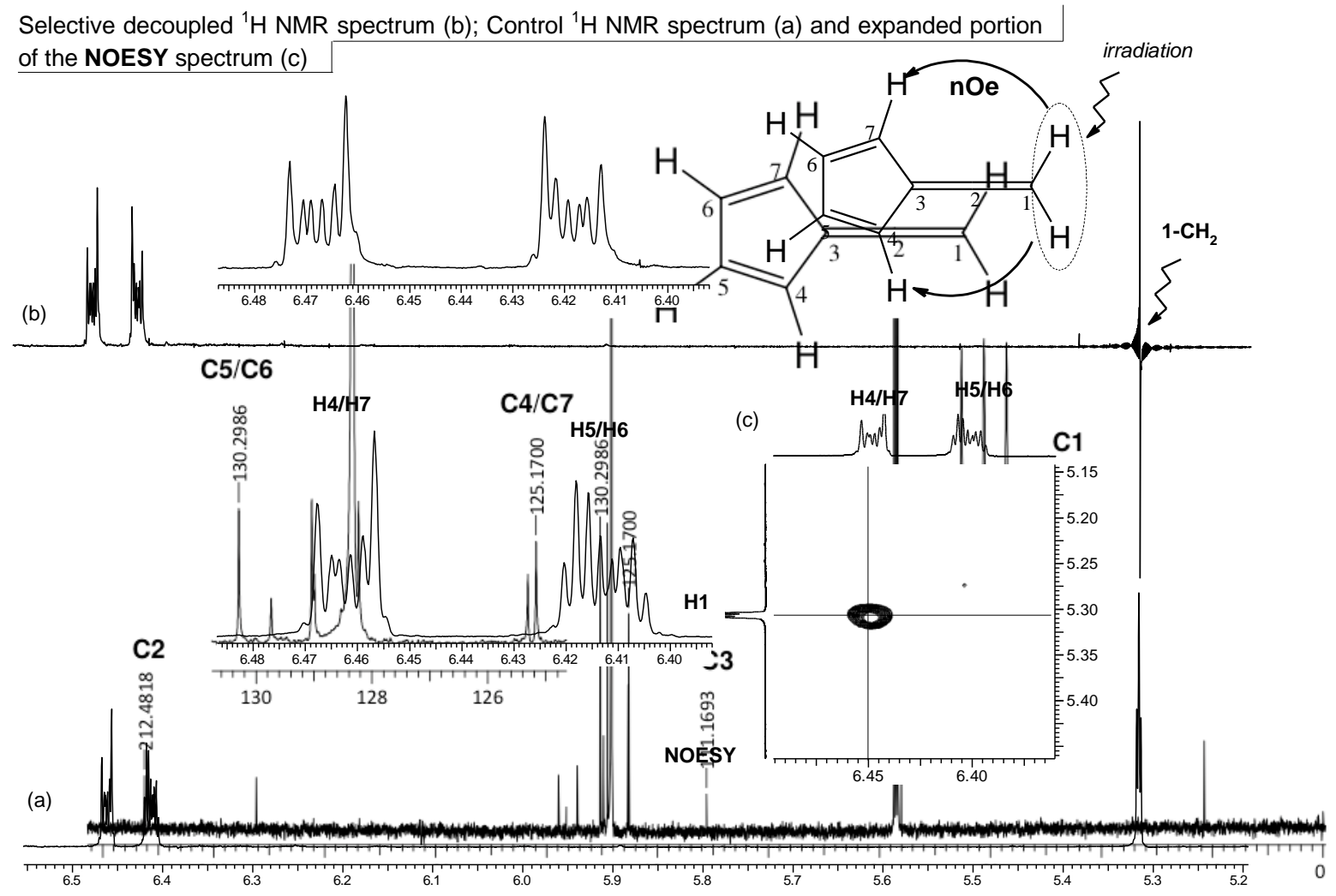

Figure 2. (a) Control 1H NMR spectrum, (b) selective decoupled 1H NMR spectrum, and (c) expanded portion of the NOESY spectrum. 


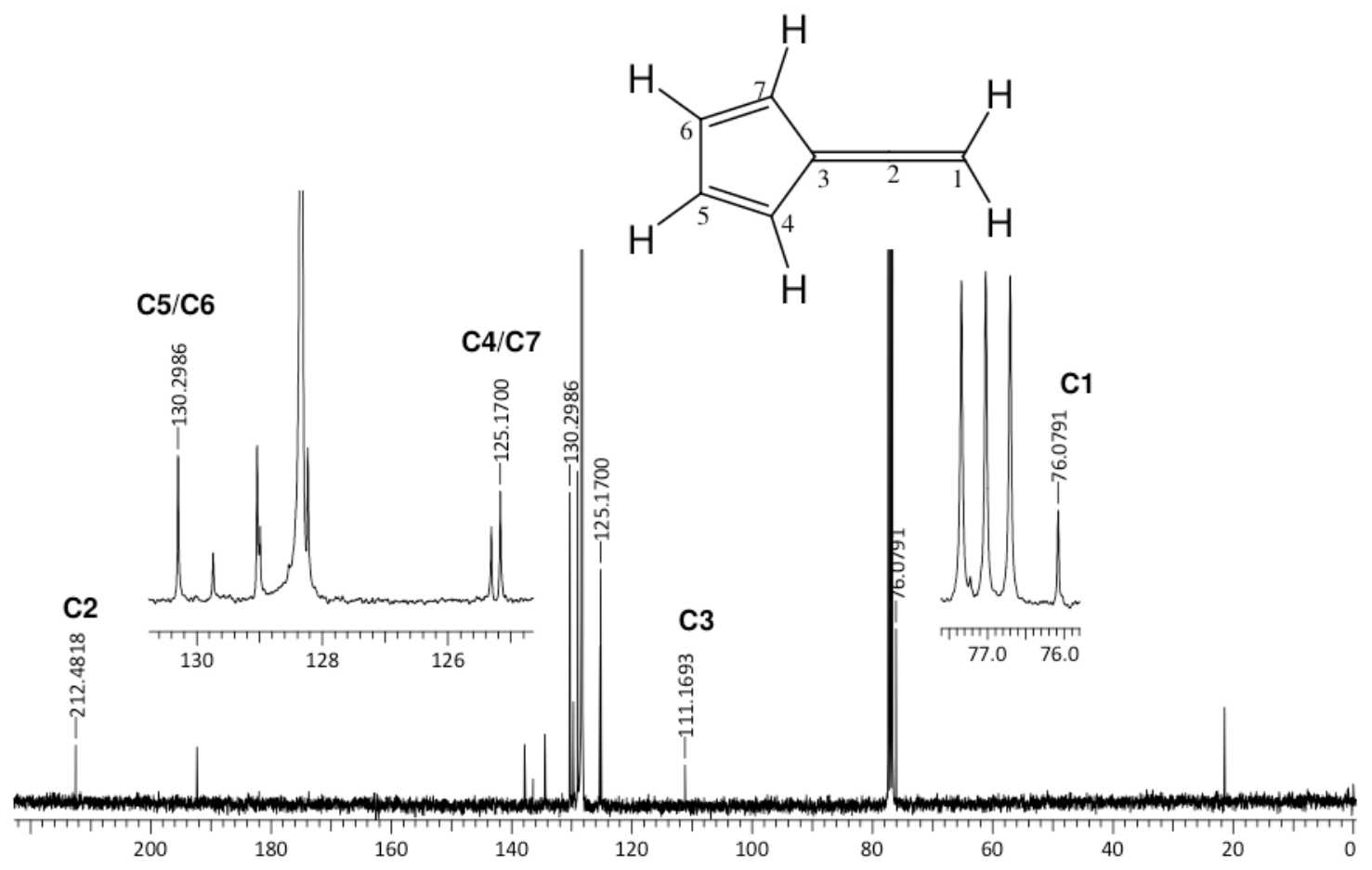

Figure 3. ${ }^{13} \mathrm{C}$ NMR spectrum of the liquid product dissolved in $\mathrm{CDCl}_{3}$.

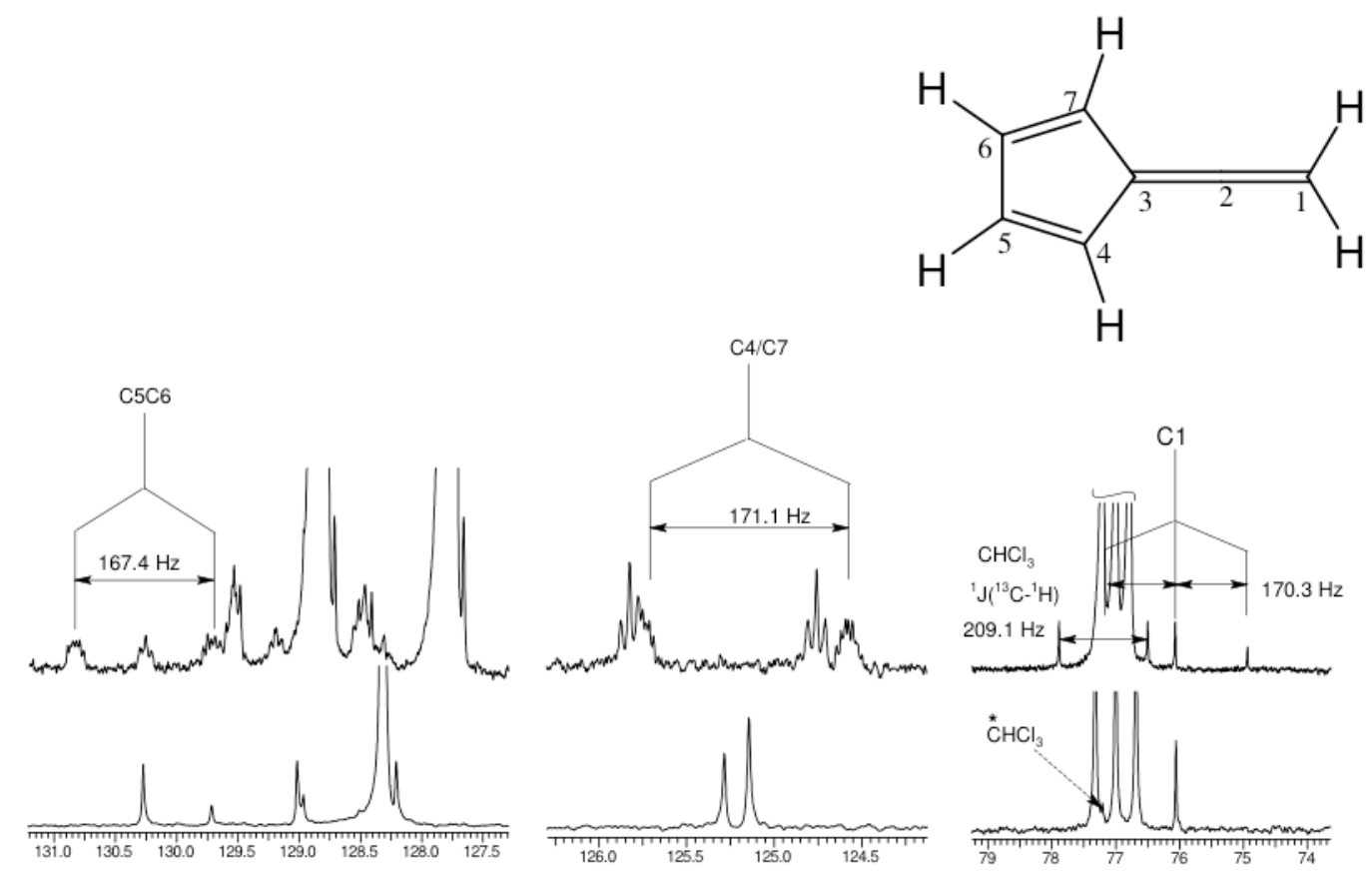

Figure 4. Proton coupled ${ }^{13} \mathrm{C}$ NMR spectrum of the liquid product dissolved in $\mathrm{CDCl}_{3}$. 


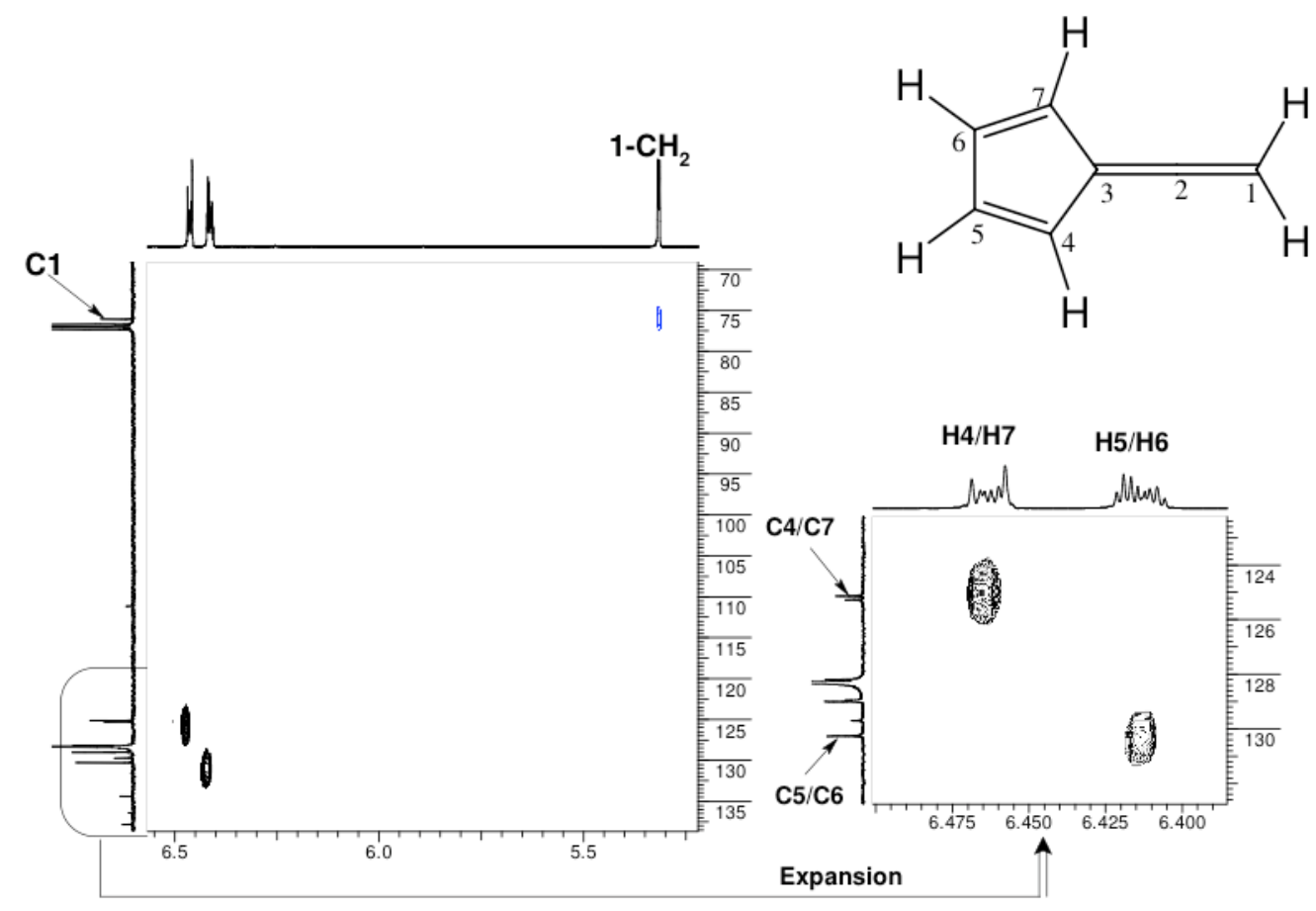

Figure 5. Two-dimensional gHSQCAD one bond correlation NMR of the fulvenallene dissolved in $\mathrm{CDCl}_{3}$.

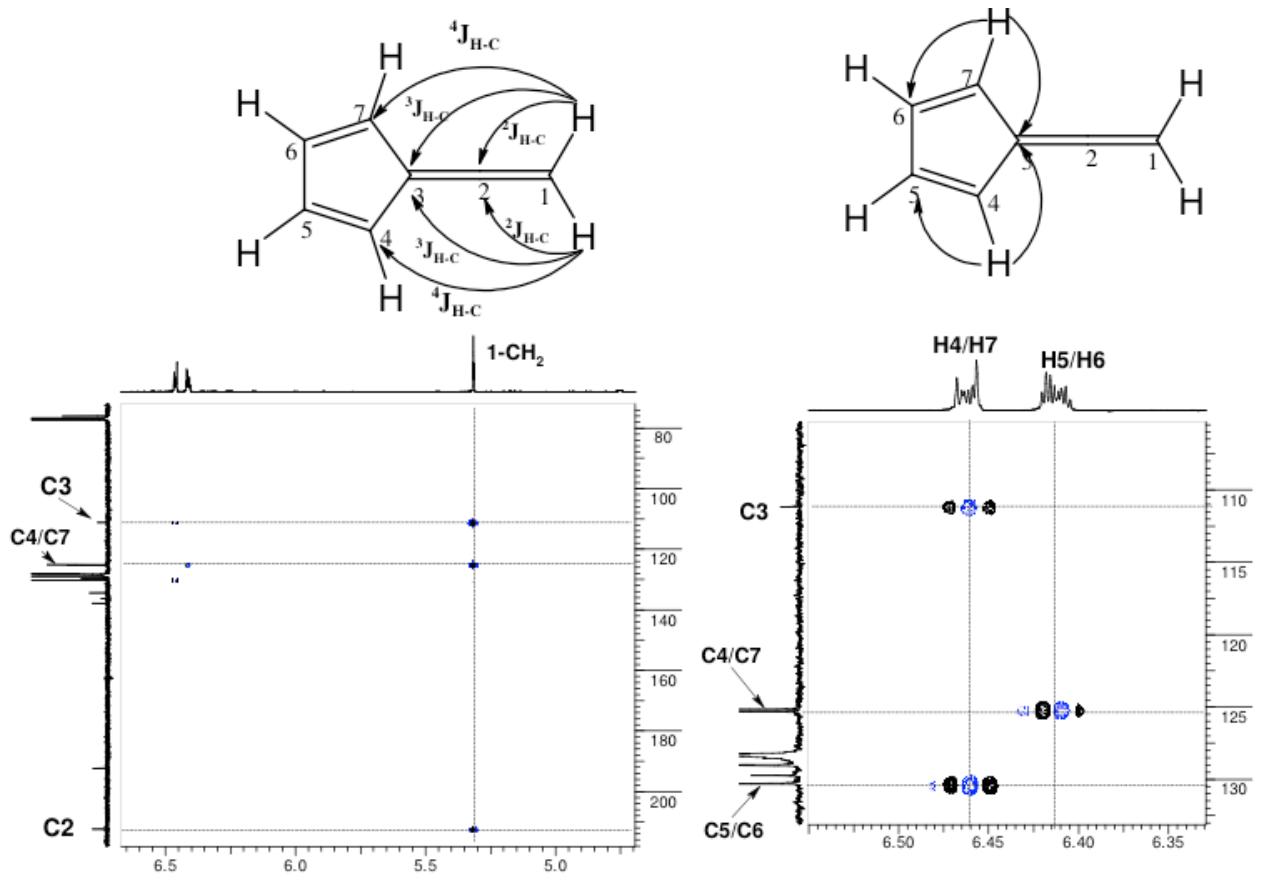

Figure 6. Two-dimensional long-range gHMBCAD correlation NMR of the fulvenallene dissolved in $\mathrm{CDCl}_{3}$. 